\title{
Hypoxia-induced muscle atrophy
}

Citation for published version (APA):

De Theye, C. C. (2016). Hypoxia-induced muscle atrophy: regulation of muscle protein turnover. [Doctoral Thesis, Maastricht University]. Maastricht University. https://doi.org/10.26481/dis.20160122cd

Document status and date:

Published: 01/01/2016

DOI:

10.26481/dis.20160122cd

Document Version:

Publisher's PDF, also known as Version of record

\section{Please check the document version of this publication:}

- A submitted manuscript is the version of the article upon submission and before peer-review. There can be important differences between the submitted version and the official published version of record.

People interested in the research are advised to contact the author for the final version of the publication, or visit the DOI to the publisher's website.

- The final author version and the galley proof are versions of the publication after peer review.

- The final published version features the final layout of the paper including the volume, issue and page numbers.

Link to publication

\footnotetext{
General rights rights.

- You may freely distribute the URL identifying the publication in the public portal. please follow below link for the End User Agreement:

www.umlib.nl/taverne-license

Take down policy

If you believe that this document breaches copyright please contact us at:

repository@maastrichtuniversity.nl

providing details and we will investigate your claim.
}

Copyright and moral rights for the publications made accessible in the public portal are retained by the authors and/or other copyright owners and it is a condition of accessing publications that users recognise and abide by the legal requirements associated with these

- Users may download and print one copy of any publication from the public portal for the purpose of private study or research.

- You may not further distribute the material or use it for any profit-making activity or commercial gain

If the publication is distributed under the terms of Article $25 \mathrm{fa}$ of the Dutch Copyright Act, indicated by the "Taverne" license above, 


\section{Hypoxia-induced muscle atrophy: regulation of muscle protein turnover}

Chiel Charles De Theye 

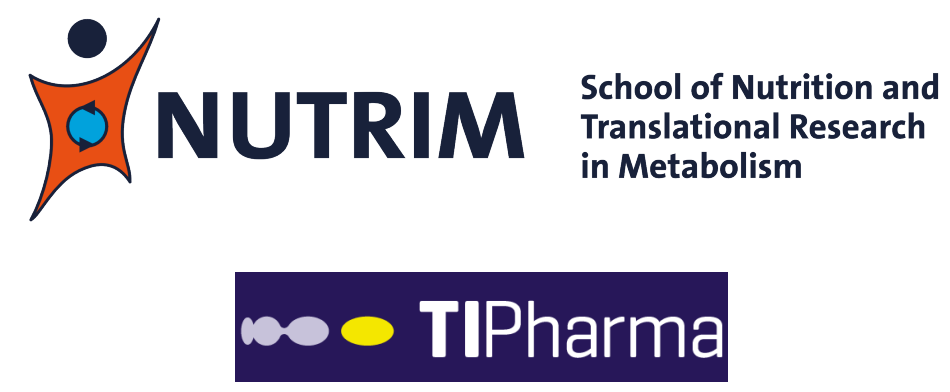

The studies presented in this thesis were performed at the NUTRIM School of Nutrition an Translational Research in Metabolism. This study was performed within the framework of the Dutch Top Institute Pharma, project T1-201.

Financial support from Stichting Pulmonologie (PUL, Maastricht) and Danone Research - Centre for Specialised Nutrition for publication of this thesis are gratefully acknowledged.

Cover design: Nicole De Theye-Hoebers

Cover image: Skeletal muscle (EDL) stained for laminin in blue, MyHC2A in green and MyHC2B in red.

Layout: $\quad$ Chiel De Theye, Nicole De Theye-Hoebers

Printed by: $\quad$ CPI - Koninklijke Wöhrmann, Zutphen

ISBN: $\quad 978-94-6203-965-0$

(C) Copyright Chiel C. De Theye, Maastricht 2015. 


\section{Hypoxia-induced muscle atrophy: regulation of muscle protein turnover}

\section{Proefschrift}

Ter verkrijging van de graad van doctor aan de Universiteit Maastricht, op het gezag van de Rector Magnificus, Prof. dr. L.L.G. Soete, volgens het besluit van het College van Decanen,

in het openbaar te verdedigen op vrijdag 22 Januari 2016 om 14:00 uur.

door

Chiel Charles De Theye

Geboren te Helmond op 27 April 1977, Nederland 


\section{Promotor}

Prof. dr. A.M.W.J. Schols

\section{Co-promotor}

Dr. R.C.J. Langen

Dr. S.E. Köhler

\section{Beoordelingscommissie}

Prof. dr. E.E. Blaak (voorzitter)

Dr. B. Blaauw, Venetian Institute of Molecular Medicine, Italy

Dr. D. Neumann

Prof. dr. L. Koenderman, Universitair Medisch Centrum Utrecht, Nederland. 


\section{Contents}

Chapter 1 General introduction 7

Adapted from manuscript: Hypoxia and muscle maintenance regulation: implications for chronic respiratory disease.

Chapter 2 Distinct responses of protein turnover-regulatory pathways in hypoxia and semi-starvation-induced muscle atrophy.

Chapter 3 Differential sensitivity of oxidative and glycolytic muscles to hypoxia-induced muscle atrophy.

Chapter 4 Hypoxia-induced muscle atrophy and impaired regulation of protein turnover are partially dependent on muscle GR signaling.

Chapter 5 Hypoxia sensitizes skeletal muscle to fasting-induced muscle atrophy and impairs AMPK/mTORC1 signaling in mice.

Chapter 6 General discussion

Summary

Samenvatting

Valorisation

Abbreviations

Dankwoord

Publications 



\section{General introduction}

Adapted from manuscript: Hypoxia and muscle maintenance regulation: implications for chronic respiratory disease

Chiel de Theije, Frédéric Costes (MD, PhD), Ramon Langen $(\mathrm{PhD})$, Christophe Pison (MD, PhD), Harry Gosker (PhD).

Current Opinion in Clinical Nutrition and Metabolic Care 2011, Nov;14(6):548553 


\section{Role of hypoxia in loss of OXPHEN and muscle mass}

Muscle wasting is a common but often under-recognized extra-pulmonary feature of chronic respiratory failure (CRF) that significantly increases disease burden. Loss of muscle mass can largely be attributed to muscle fibre atrophy, particularly of type II fibres [1, 2]. In addition to the loss of muscle mass, peripheral muscles of patients with chronic obstructive pulmonary disease (COPD) often are characterized by a so-called loss of oxidative phenotype (OXPHEN); a shift from slow oxidative type I fibres towards fast glycolytic type II fibres, reduced oxidative enzyme capacities and mitochondrial impairments [3,4]. In addition to increased fatigability, loss of muscle OXPHEN may contribute to elevated energy requirements (since oxidative energy metabolism is more efficient than glycolytic energy metabolism) and to enhanced oxidative stress, thereby augmenting the onset or progression of muscle wasting [5]. Hypoxemia, either chronic or intermittent, is an obvious feature of respiratory failure, but surprisingly its potential impact on muscle maintenance in CRF patients is rather unexplored.

What indications do we actually have that hypoxia is involved in muscle pathology in respiratory disease? The first probably came from Jakobsson et al. who reported low percentages of type I fibres that were associated with low arterial oxygen pressures in COPD [6]. Impaired muscle OXPHEN in COPD has been a consistent finding since then, but strong evidence for the involvement of hypoxia is lacking simply because this has not been further studied in groups of patients with severe hypoxemia (partial pressure of oxygen in arterial blood (PaO2 $<7.3 \mathrm{kPa}$ ). In patients with restrictive lung disease and mild hypoxemia related to scoliosis, Swallow et al. showed that impaired muscle function was associated with a decreased proportion of type I fibres and increased oxidative stress [7]. Pulmonary arterial hypertension is also an important cause of chronic hypoxemia and skeletal muscle abnormalities have indeed been reported in these patients as well, including a decreased in type I fibres and slightly reduced oxidative enzymes [8].

Additional arguments in favour of a role for hypoxia in impaired muscle maintenance come from interventions aimed at alleviating hypoxemia. Jakobsson et al. found signs of improved muscle oxidative metabolism after long-term oxygen therapy (LTOT) [9]. Lung volume reduction surgery (LVRS) represents a functional treatment for emphysema, which improves respiratory mechanics and reduces dyspnea. Mineo et al. showed improvements in muscle mass that were maintained for at least 5 years following LVRS and were associated with improved outcomes [10]. Interestingly, they also reported a decrease in plasma inflammatory markers such as tumor necrosis factor alpha (TNFa), interleukin 6 (IL-6) and interleukin 8 (IL-8) 1 year after surgery [11], pointing towards the existence of hypoxia-induced systemic inflammation. After lung transplantation, and thereby 
correction of hypoxemia, only partial restoration of muscle OXPHEN has been reported, suggesting a long-lasting mark of chronic hypoxia that could only partially be reversed by rehabilitation [12-14].

\section{Hypoxia and maintenance of muscle mass}

Observational studies in humans showed that long-term exposure to high altitude resulted in loss of limb muscle mass, which has been described as an adaptive mechanism to improve muscle oxygenation by a relatively increased capillarization $[15,16]$. Muscle atrophy also occurs in animals exposed to experimental hypoxia [17, 18]. The balance between protein synthesis and degradation is an important determinant of the maintenance of skeletal muscle mass (see Figure 1 for a schematic overview). Although most experimental work on hypoxia-induced protein turnover has thus far focused on regulation of protein synthesis by hypoxia, effects on ubiquitin (UB) 26S-proteasome mediated protein degradation, as well as autophagy have also been described. As most of this work has been performed in non-muscle cells and tissues, extrapolation of these results to skeletal muscle protein turnover must be done with care. Control of transcription can influence the rate of protein synthesis and can be regulated by transcription factors. Hypoxia-inducible factors (HIFs) are recognized as a key modulators of the transcriptional response during hypoxic stress and are involved in many adaptive responses including protein synthesis [19]. The rate of mRNA translation is mainly controlled during the initiation phase by eukaryotic translation initiation factors (elFs). elF2 $\alpha$ is permissive to mRNA translation in the nonphosphorylated state, but blocks the initiation of protein synthesis once it becomes phosphorylated by one of the 4 stress kinases which are, among others, activated by hypoxia [20], and oxidative and endoplasmic reticulum stress [21]. Another elF, elF4E is inhibited when bound by elF4E-binding protein 1 (4E-BP1). Phosphorylation of 4E-BP1 by the mammalian target of rapamycin (mTOR) in the mTOR complex 1 (mTORC1), results in dissociation of elF4E from 4E-BP1 and the formation of the translation-initiation complex [22]. mTOR also activates ribosomal protein S6 kinase beta-1 (P70S6K1), which in turn, phosphorylates the ribosomal protein S6 (S6) and, thus, stimulates translation. Hypoxia reduces phosphorylation of mTOR and its downstream effectors 4E-BP1 and P70S6K1, and thus inhibits protein synthesis [23]. mTORC1 activity is controlled by the tuberous sclerosis protein 1 and 2 (TSC1/TSC2) complex, which is regulated by hypoxia-sensitive pathways, including AMP-activated protein kinase (AMPK), protein kinase B (PKB, a.k.a. AKT) and DNA-damage-inducible transcript 4 protein (DDIT4/REDD1). AMPK phosphorylates and thereby stimulates TSC2 and, as a result, inactivates mTORC1 [24]. The degree of AMPK activation depends on the severity of the hypoxic conditions [25]. Phosphorylated active AKT deac- 
tivates TSC2 through phosphorylation at Ser939 and Thr1462, which results in binding of TSC2 to the inhibitory 14-3-3 complex [26, 27]. Conversely, increased Redd1 expression results activation of TSC2 by its dissociation from 14-3-3, which subsequently inhibits mTORC1 in response to hypoxia [17, 28]. Increased Redd1 expression during hypoxia results from increased expression of the transcription factors activating transcription factor 4 (ATF4) and CCAAT/enhancerbinding protein- $\beta$ (C/EBP- $\beta$ ) [25, 29], HIF1 $\alpha$ [30]. Hypoxia-induced expression of Atf 4 and $C / E B P-\beta$ is a result of the development of endoplasmic reticulum stress and signaling via elF2 $\alpha[25,29]$. AKT is a serine/threonine protein kinase and a key protein in the regulation of muscle mass, which controls protein synthesis via mTOR, but also regulates protein degradation. AKT activation by phosphoinositide 3-kinase (PI3K) is involved in the stimulation of myogenic differentiation by insulin growth factor-1 (IGF-1). During hypoxia, this myogenic response changes into a mitogenic response by redirection of IGF-1 signaling to mitogen-activated protein kinase (MAPK) instead of AKT activation. This finding implies that oxygen gradients may be of importance for myogenesis. It is currently unknown via which mechanisms hypoxia influences AKT phosphorylation, although HIF1a may play a role [19].

Increased protein degradation in skeletal muscle results from increased lysosomal and/or proteasomal protein degradation. Autophagy can be initiated in response to nutritional depletion or hypoxia via activation of HIF1 $\alpha$ and/or endoplasmic reticulum stress via BCL2/adenovirus E1B $19 \mathrm{kDa}$ protein-interacting protein 3 (BNIP3) and microtubule-associated protein 1 light chain-3 (LC3) [31, 32]. Autophagy, which captures organelles and proteins in autophagic vacuoles $(\mathrm{AV})$, relies on lysosomal protein degradation and is important in muscle maintenance [33]. Muscle protein degradation is also controlled by the ubiquitin-proteasome system (UPS). In this pathway the E3 Ub-ligases, muscle-specific ring finger 1 (MURF1) and muscle atrophy F-box (ATROGIN-1/MAFbx), label muscle proteins with poly-ubiquitin chains, resulting in their targeted degradation by the 26S-proteasome. The expression of both Murf1 and Atrogin-1 are increased during hypoxia [34, 35]. The expression of these atrogenes is regulated by inducible transcription factors like nuclear factor kappa-light-chain-enhancer of activated B-cells (NF-KB) [36] and Forkhead box O (FOXO). FOXO1 activity is negatively controlled by AKT-mediated phosphorylation, which results in its nuclear export and subsequent suppression of the transcription of Murf1 and Atrogin-1 resulting in decreased protein degradation [37, 38]. However, the mechanisms by which hypoxia controls expression of these atrogenes are still unclear. The regulation of AKT activity may be the key in regulating muscle mass during hypoxic conditions. Myostatin (MSTN) signaling is able to suppress AKT activation, which in turn decreases protein synthesis via mTOR and de-repression of FOXO1-mediated 
atrogene transcription [39, 40]. Mstn expression is increased in muscle atrophy during hypoxic conditions in humans, rats and muscle cells [18]. Protein synthesis and degradation are regulated by complex mechanisms, which allow rapid adaptation to acute hypoxic stress. However, in response to sustained hypoxic stress, adaptive mechanisms may not be adequate in maintaining the balance in muscle protein turnover, which may culminate in muscle atrophy in chronic disease.

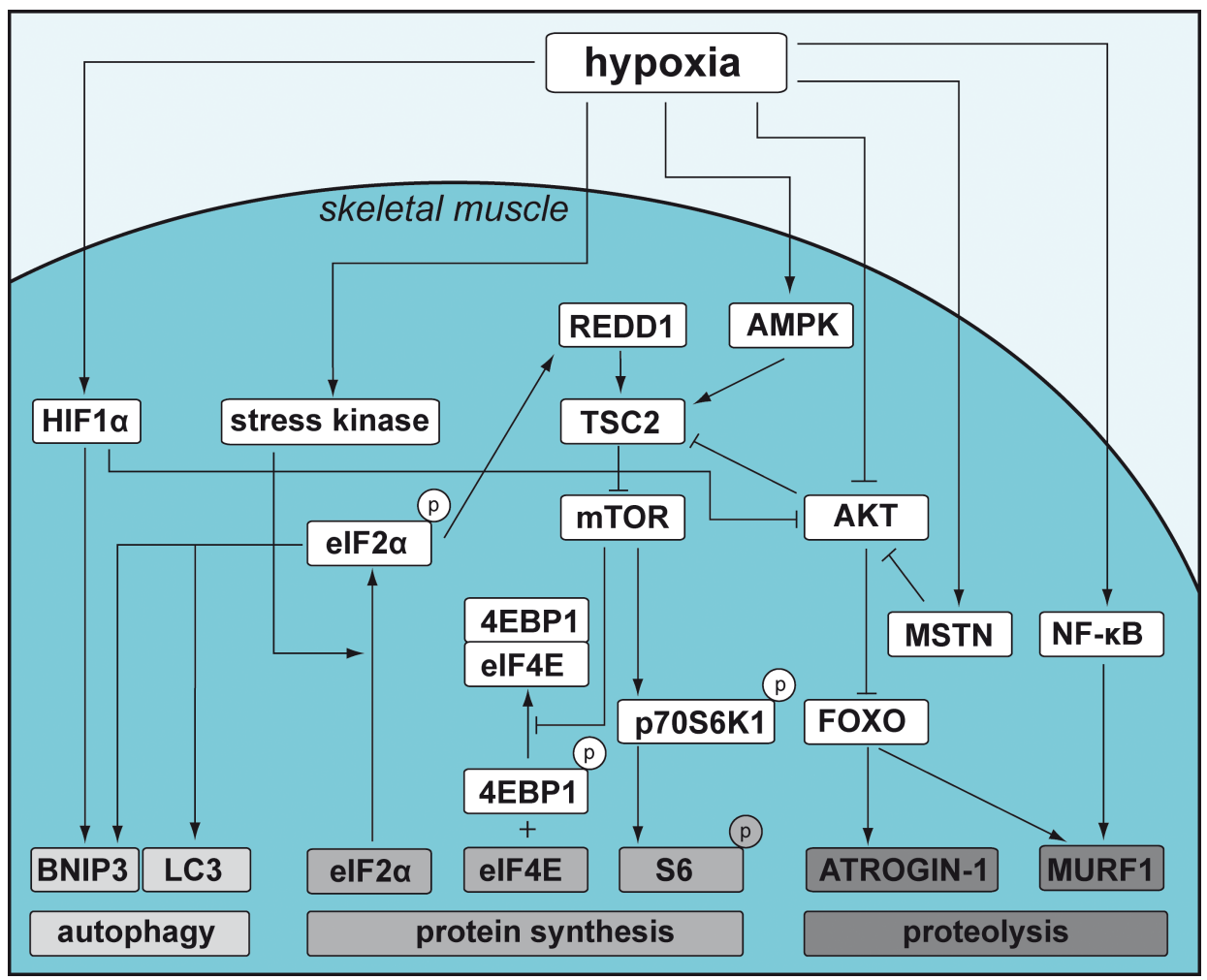

Figure 1. Schematic overview of potential direct or indirect hypoxia signals with respect to the

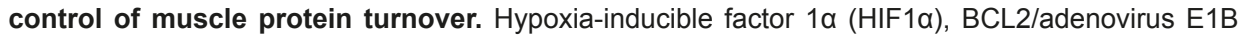
$19 \mathrm{kDa}$ protein-interacting protein 3 (BNIP3), microtubule-associated protein 1 light chain-3 (LC3), eukaryotic translation initiation factor $2 \alpha$, DNA-damage-inducible transcript 4 protein (REDD1), tuberous sclerosis protein 2 (TSC2), mammalian target of rapamycin (mTOR), elF4E-binding protein 1 (4EBP1), eukaryotic initiation factor 4E (elF4E), ribosomal protein S6 kinase beta-1 (P70S6K1), ribosomal protein S6 (S6), AMP-activated protein kinase (AMPK), serine/threonine protein kinase AKT (AKT), Myostatin (MSTN), Forkhead box O (FOXO), nuclear factor kappa-light-chain-enhancer of activated $B$ cells (NF-KB), muscle atrophy F-box (Atrogin-1), muscle-specific ring finger 1 (MURF1). 


\section{Hypoxia and maintenance of muscle metabolism}

Literature is rather inconsistent regarding the long-term response of muscle metabolic profile to hypoxia. The adaptations of muscle in humans exposed to high altitude have been extensively studied and the overall consensus is that muscle adapts to high altitude hypoxia by a decrease in oxidative capacity, whereas when combined with exercise training, muscle OXPHEN may even improve [4143]. Although yet to be confirmed, it is possible that these discrepancies were caused by differences in age as only the younger animals seemed to exhibit loss of muscle OXPHEN [44]. By using an in vitro model in which cultured muscle cells were exposed to low oxygen levels the pure effect of hypoxia alone could be studied, which revealed a hypoxia-induced down regulation of the expression of mitochondrial proteins [45]. The exact mechanisms underlying hypoxia-induced loss of muscle OXPHEN also remain largely unclear. There are a few indications that point towards involvement of HIF1 $\alpha$ which indisputably is an important enhancer of glycolytic metabolism, likely at the expense of oxidative metabolism. Muscles of patients with Chuvash polycythemia, a disease with a genetic abnormality resulting in an impaired HIF1a degradation resulting in elevated HIF1a levels at normal oxygen tensions, exhibited early and accelerated phosphocreatine depletion accompanied by increased acidosis and lactate accumulation during exercise, indicative of impaired muscle OXPHEN [46]. Furthermore, higher mitochondrial enzyme activities have been observed in muscles of mice lacking HIF1a [47]. Another potential way of adjusting mitochondrial capacity to hypoxia is mitochondrial autophagy (mitophagy), which also requires HIF1a [48]. In rats, Hif1 a expression was highest in the fast-twitch type II glycolytic muscles [49]. This study also showed elevated HIF1 $\alpha$ levels in muscles electrically stimulated with a high frequency (inducing fast fibre type-specific genes) and reduced HIF1a levels after low-frequency stimulation (inducing slow fibre type-specific genes), stressing the role of HIF1a in muscle OXPHEN regulation even in the absence of hypoxia. It is, however, unclear whether these results can be extrapolated to humans, since HIF1a protein expression was found to be highest in oxidative rather than glycolytic muscles [50]. The peroxisome proliferator-activated receptors (PPARs) and in particular their co-activator peroxisome proliferator-activated receptor gamma coactivator 1-alpha (PGC1 1 ) are key regulators of OXPHEN in muscle $[51,52]$. In vitro studies showed that hypoxia can certainly impair components of the PPAR pathway at both transcriptional and post-transcriptional levels in cultured muscle cells [48], feasibly leading to loss of muscle OXPHEN. Controversially, PGC1 $\alpha$ has been shown to induce the expression of typical HIF1a target genes (especially those involved in angiogenesis, like vascular endothelial growth factor (VEGF)) at physiological oxygen levels [53]. A plausible explanation for this apparent paradox might simply be the fact that PGC1a-driven mito- 
chondrial biogenesis leads to an increased oxygen demand which is not (yet) matched by the oxygen supply and hence intracellular hypoxia occurs, triggering HIF1a-dependent gene expression [54]. Alternatively, as NF-KB has also been implicated in hypoxia-signaling, it can be speculated that NF-KB also mediates hypoxia-induced loss of muscle OXPHEN. NF-KB is a family of proteins involved in innate immunity, inflammation and apoptosis. Its upstream regulation involves formation of a nuclear factor of kappa light polypeptide gene enhancer in B-cells inhibitor (IKB) kinase complex, which through its kinase activity ultimately results in NF-KB transcriptional activity [55]. Remels et al recently showed that NF-KB activation indeed impairs muscle OXPHEN [56], although it remains to be established whether this also occurs under hypoxia. As indicated above, HIF1a expression depends on muscle composition, which may results in different responses of glycolytic and oxidative muscle to atrophy stimuli. Differential sensitivity of these muscles to atrophy stimuli other than hypoxia has previously been observed in animal models [57-59]. Rats that were exposed for 10 weeks to hypobaric hypoxia also showed a selective reduction of the fibre cross sectional area (FCSA) of the glycolytic EDL [60]. Nevertheless a clear mechanism for the differential sensitivity of muscle has been elusive. Recapitulated, there are clear indications that hypoxia can impair muscle OXPHEN, but it remains to be clarified under what specific conditions this happens, which mechanisms are responsible and how muscle composition may influence the response.

\section{Molecular sensors of hypoxia}

Probably the most important regulators of cell-autonomous responses to hypoxia belong to the HIF family of transcription factors [41]. These factors are heterodimeric proteins composed of a HIF $\alpha$ (HIF1-3a and of which HIF1 $\alpha$ is best described) and a HIF $\beta$ subunit. The Hif1 $\alpha$ gene is continuously expressed, but under normoxic conditions the protein is rapidly hydroxylated by specific prolyl hydroxylases (PHDs), enabling binding of the E3 ligase von Hippel-Lindau (VHL) leading to degradation by the ubiquitin proteasome pathway. As oxygen levels drop, so does the rate of hydroxylation, thereby allowing the build-up of transcriptionally active HIF proteins, driving the expression of their target genes which are mainly involved in glycolytic metabolism and angiogenesis [41]. Evidence is accumulating that the above-mentioned PHDs can also modulate NF-KB signaling [61, 62], indicative of hypoxia signaling independent of HIF1 $\alpha$. Interestingly, evidence for a cross-talk between HIF1 $\alpha$ and NF-KB is also emerging [61, 62]: although HIF1 $\alpha$ is primarily post-transcriptionally regulated, NF-KB has been reported to upregulate Hif1a gene expression and that vice versa HIF activates NF-KB signaling. Given these facts, a primary regulatory role of the HIF and NF$\mathrm{KB}$ signaling pathways, orchestrated by the PHDs, seems therefore credible in 
hypoxia-induced alterations in muscle maintenance.

As discussed above, the PPARs and PGC1 $\alpha$ are key OXPHEN regulators, which may be under negative control of hypoxia. Reduced expression levels of these regulators have indeed been shown in muscles of patients with COPD [63]. Regarding muscle atrophy, there are some indications that in muscles of COPD patients markers of the ubiquitin proteasome pathway, including the atrogenes MURF1 and ATROGIN-1, are increased [64, 65]. Moreover, COPD exacerbations are frequently associated with augmented hypoxemia. Fascinatingly, it has recently been shown that the gene expression of these atrogenes was upregulated, whereas OXPHEN expression was down-regulated in patients experiencing an exacerbation when compared to stable COPD patients [66]. Finally, increased expression levels of Mstn have been reported for COPD patients characterized by hypoxemia [18]. It is tempting to attribute all these findings to hypoxemia, but a definitive conclusion is impossible. To add to the ambiguity, the involvement of the putative hypoxia sensors HIF and NF-KB in COPD-related muscle patho logy also remains unclear: Muscle HIF gene expression tended to be increased in COPD, but on the other hand, the expression of the E3 ligase VHL, which targets HIFa's for degradation, was also increased [67]. Increased muscle NF$\mathrm{KB}$ activation has indeed been reported in COPD patients with low body weight [68], although unaltered muscle NF-kB activation has recently been observed in patients with atrophy [65]. The influence of chronic hypoxemia was more specifically studied by Favier et al, who reported a down-regulation of the anabolic AKT/ mTOR pathway and a tendency towards an up-regulation of its putative inhibitor REDD1 in hypoxemic versus normoxemic patients with COPD [17]. In parallel to the above-mentioned direct effects of hypoxia on muscle maintenance, indirect mechanisms may also be involved in COPD. Intriguingly, a correlation between the degree of hypoxemia and circulating TNFa in patients with COPD was observed [69], suggesting hypoxia-induced systemic inflammation [70], which in turn may contribute to muscle pathology. In addition, loss of appetite and subsequent malnutrition can result in muscle-atrophy as seen in anorexic patients [71] and fasting-induced muscle atrophy animal models [72-75]. In patients with advanced COPD loss of appetite and subsequent cachexia is common. This may be related to hypoxia-induced release of leptin, a hormone that attenuates appetite. Indeed, elevated circulating leptin levels have been reported in cachectic COPD patients with more severe hypoxemia [76] and in COPD patients suffering from an acute exacerbation [77]. In particular during acute exacerbations patients may be extra vulnerable as the effects of hypoxia and strongly reduced food intake on muscle mass maintenance converge. Hypoxemia and reduced food intake per se have been associated with elevated glucocorticoid (GC) concentrations and glucocorticoid receptor (GR) signaling [78-83]. GR signaling is involved 
in muscle mass regulation through genomic and non-genomic effects [84]. Genomic effects include activation of protein degradation by up-regulation of genes involved in UPS, such as Foxo1, Murf1 and Atrogin-1 [74, 85, 86] and Krüppellike factor 15 (KIf15) expression. KLF15 and GR target REDD1 also contributes to the regulation of protein synthesis $[87,88]$. Non-genomic GR actions involve suppression of protein synthesis through the inhibition of the PI3K/AKT/TSC1-2/ mTOR signaling pathway, in which GR activation prevents association of PI3K with insulin receptor substrate-1 (IRS-1) [89]. However, GC/GR signaling has been studied in reduced food intake (fasting)-associated muscle atrophy [90], but not in hypoxia-induced muscle atrophy.

\section{Aim of the thesis}

Although the impact of hypoxia on muscle mass maintenance has been investigated, these studies have mainly addressed the cell-autonomous effects of hypoxia on skeletal muscle using in vitro approaches. In patients, hypoxemia, or the associated reduced food intake may affect multiple organs and tissues simultaneously, resulting in more than only direct effects of tissue hypoxia on skeletal muscle: effectors such as hormones may be secreted, which may affect muscle mass maintenance. In order to understand the influence of hypoxia on mass regulation of skeletal muscle and the interaction with other organs, we developed a normobaric hypoxia mouse model. In this model we investigated the adaptation of skeletal muscle mass regulation during acute (day 2 and 4) and chronic (day 21) hypoxia by investigating changes in muscle mass and the regulation of protein turnover in the gastrocnemius muscle. In chapter 2 , we hypothesized that normobaric hypoxia induces muscle atrophy, which is not completely attributable to semi-starvation. Furthermore, we evaluated whether hypoxia-induced muscle atrophy involves a coordinated increase in muscle proteolysis signaling and a decrease of regulatory signals for protein synthesis. As mentioned above, there may be a differential sensitivity of skeletal muscle fibre types to hypoxia [60]. As the exact mechanisms behind this differential sensitivity remains elusive, we investigated adaptations in skeletal muscle morphology and signaling pathways related to protein degradation in chapter 3 . We hypothesized that oxidative muscle fibres are more sensitive to hypoxia-induced muscle atrophy compared to glycolytic muscle fibres. From these studies, a potential role of the glucocorticoids and glucocorticoid receptor (GR) signaling in hypoxia-induced muscle atrophy became apparent (chapter 1-3). Therefore, in chapter 4, we further investigated the involvement of GR in hypoxia-induced muscle atrophy using a skeletal musclespecific GR knockout mouse model. We hypothesized that muscle GR signaling in response to elevated circulating corticosterone concentrations is required for increased protein degradation cues and affects the regulation of protein synthe- 
sis signaling during hypoxia-induced muscle atrophy. In previous chapters, food intake appeared to be a major factor controlling protein turnover independently of hypoxia. Furthermore, as dietary intake may drop dramatically in CRF patients during an acute disease exacerbation, we questioned whether and how hypoxia would influence fasting-induced muscle atrophy and protein turnover regulation. In chapter $\mathbf{5}$ we hypothesized that hypoxia sensitizes skeletal muscle to fastinginduced skeletal muscle atrophy through an impairment of muscle protein turnover signaling. Finally, in chapter 6 the experimental findings are integratively discussed and directions for future research are pointed out. 


\section{References}

1. Eliason G, Abdel-Halim S, Arvidsson B, Kadi F, Piehl-Aulin K. Physical performance and muscular characteristics in different stages of COPD. Scandinavian journal of medicine \& science in sports. 2009;19(6):865-70.

2. Gosker HR, Engelen MPKJ, van Mameren H, van Dijk PJ, van der Vusse GJ, Wouters EFM, et al. Muscle fiber type IIX atrophy is involved in the loss of fat-free mass in chronic obstructive pulmonary disease. Am J Clin Nutr. 2002;76:113-9.

3. Gosker HR, Zeegers MP, Wouters EF, Schols AM. Muscle fibre type shifting in the vastus lateralis of patients with COPD is associated with disease severity: a systematic review and meta-analysis. Thorax. 2007;62(11):944-9.

4. Rabinovich RA, Vilaró J. Structural and funtional changes of peripheral muscles in COPD patients. Lung. 2010;i:123-33.

5. Schols AM, Gosker HR. The pathophysiology of cachexia in chronic obstructive pulmonary disease. Curr Opin Support Palliat Care. 2009;3(4):282-7.

6. Jakobsson P, Jorfeldt L, Brundin A. Skeletal muscle metabolites and fibre types in patients with advanced chronic obstructive pulmonary disease (COPD), with and without chronic respiratory failure. The European respiratory journal. 1990;3(2):192-6.

7. Swallow EB, Barreiro E, Gosker H, Sathyapala SA, Sanchez F, Hopkinson NS, et al. Quadriceps muscle strength in scoliosis. The European respiratory journal. 2009;34(6):1429-35.

8. Mainguy V, Maltais F, Saey D, Gagnon P, Martel S, Simon M, et al. Peripheral muscle dysfunction in idiopathic pulmonary arterial hypertension. Thorax. 2010;65(2):113-7.

9. Jakobsson P, Jorfeldt L. Long-term oxygen therapy may improve skeletal muscle metabolism in advanced chronic obstructive pulmonary disease patients with chronic hypoxaemia. Respiratory medicine. 1995;89(7):471-6.

10. Mineo D, Ambrogi V, Lauriola V, Pompeo E, Mineo TC. Recovery of body composition improves long-term outcomes after lung volume reduction surgery for emphysema. The European respiratory journal. 2010;36(2):408-16.

11. Mineo D, Ambrogi V, Cufari ME, Gambardella S, Pignotti L, Pompeo E, et al. Variations of inflammatory mediators and alpha1-antitrypsin levels after lung volume reduction surgery for emphysema. American journal of respiratory and critical care medicine. 2010;181(8):806-14.

12. Evans AB, Al-Himyary AJ, Hrovat MI, Pappagianopoulos P, Wain JC, Ginns LC, et al. Abnormal skeletal muscle oxidative capacity after lung transplantation by 31P-MRS. American journal of respiratory and critical care medicine. 1997;155(2):615-21.

13. Guerrero K, Wuyam B, Mezin P, Vivodtzev I, Vendelin M, Borel JC, et al. Functional coupling of adenine nucleotide translocase and mitochondrial creatine kinase is enhanced after exercise training in lung transplant skeletal muscle. American journal of physiology Regulatory, integrative and comparative physiology. 2005;289(4):R1144-54.

14. Wang XN, Williams TJ, McKenna MJ, Li JL, Fraser SF, Side EA, et al. Skeletal muscle oxidative capacity, fiber type, and metabolites after lung transplantation. American journal of respiratory and critical care medicine. 1999;160(1):57-63. 
15. Boyer SJ, Blume FD. Weight loss and changes in body composition at high altitude. Journal of applied physiology: respiratory, environmental and exercise physiology. 1984;57:1580-5.

16. Hoppeler H, Kleinert E, Schlegel C, Claassen H, Howald H, Kayar SR, et al. Morphological adaptations of human skeletal muscle to chronic hypoxia. Int J Sports Med. 1990;11 Suppl 1:S3-9.

17. Favier FB, Costes F, Defour A, Bonnefoy R, Lefai E, Baugé S, et al. Down-regulation of Akt/ mammalian target of rapamycin pathway in skeletal muscle is associated with increased REDD1 expression in response to chronic hypoxia. American journal of physiology Regulatory, integrative and comparative physiology. 2010:1659-66.

18. Hayot M, Rodriguez J, Vernus B, Carnac G, Jean E, Allen D, et al. Myostatin up-regulation is associated with the skeletal muscle response to hypoxic stimuli. Molecular and cellular endocrinology. 2010.

19. Ren H, Accili D, Duan C. Hypoxia converts the myogenic action of insulin-like growth factors into mitogenic action by differentially regulating multiple signaling pathways. Proc Natl Acad Sci U S A. 2010;107:5857-62.

20. Koritzinsky M, Rouschop KMa, van den Beucken T, Magagnin MG, Savelkouls K, Lambin P, et al. Phosphorylation of elF2alpha is required for mRNA translation inhibition and survival during moderate hypoxia. Radiotherapy and oncology : journal of the European Society for Therapeutic Radiology and Oncology. 2007;83:353-61.

21. Koumenis C, Naczki C, Koritzinsky M, Rastani S, Diehl A, Sonenberg N, et al. Regulation of Protein Synthesis by Hypoxia via Activation of the Endoplasmic Reticulum Kinase PERK and Phosphorylation of the Translation Initiation Factor elF2 $\square$. Society. 2002;22:7405-16.

22. Ma XM, Blenis J. Molecular mechanisms of mTOR-mediated translational control. Nat Rev Mol Cell Biol. 2009;10:307-18.

23. Arsham AM, Howell JJ, Simon MC. A novel hypoxia-inducible factor-independent hypoxic response regulating mammalian target of rapamycin and its targets. J Biol Chem. 2003;278:2965560.

24. Inoki K, Zhu T, Guan K-L. TSC2 mediates cellular energy response to control cell growth and survival. Cell. 2003;115:577-90.

25. Liu L, Cash TP, Jones RG, Keith B, Thompson CB, Simon MC. Hypoxia-induced energy stress regulates mRNA translation and cell growth. Mol Cell. 2006;21:521-31.

26. Cai SL, Tee AR, Short JD, Bergeron JM, Kim J, Shen J, et al. Activity of TSC2 is inhibited by AKT-mediated phosphorylation and membrane partitioning. The Journal of cell biology. 2006;173(2):279-89.

27. Miyazaki M, McCarthy JJ, Esser KA. Insulin like growth factor-1-induced phosphorylation and altered distribution of tuberous sclerosis complex (TSC)1/TSC2 in C2C12 myotubes. Febs J. 2010;277(9):2180-91.

28. DeYoung MP, Horak P, Sofer A, Sgroi D, Ellisen LW. Hypoxia regulates TSC1/2-mTOR signaling and tumor suppression through REDD1-mediated 14-3-3 shuttling. Genes \& development. 2008;22:239-51. 
29. Jin HO, Seo SK, Woo SH, Kim ES, Lee HC, Yoo DH, et al. Activating transcription factor 4 and CCAAT/enhancer-binding protein-beta negatively regulate the mammalian target of rapamycin via Redd1 expression in response to oxidative and endoplasmic reticulum stress. Free Radic Biol Med. 2009;46(8):1158-67.

30. Shoshani T, Faerman A, Mett I, Zelin E, Tenne T, Gorodin S, et al. Identification of a novel hypoxia-inducible factor 1-responsive gene, RTP801, involved in apoptosis. Mol Cell Biol. 2002;22(7):2283-93.

31. Bellot G, Garcia-Medina R, Gounon P, Chiche J, Roux D, Pouysségur J, et al. Hypoxia-induced autophagy is mediated through hypoxia-inducible factor induction of BNIP3 and BNIP3L via their BH3 domains. Mol Cell Biol. 2009;29:2570-81.

32. Rouschop KMA, Beucken TVD, Dubois L, Niessen H, Bussink J, Savelkouls K, et al. The unfolded protein response protects human tumor cells during hypoxia through regulation of the autophagy genes MAP1LC3B and ATG5. Cancer. 2010;120:127-41.

33. Sandri M. Autophagy in skeletal muscle. FEBS letters. 2010;584(7):1411-6.

34. Cannata DJ, Ireland ZOE, Dickinson H, Snow RODJ, Russell AP, West JANM, et al. Maternal Creatine Supplementation From Mid-Pregnancy Intrapartum Hypoxia-Induced Damage. Pediatric Research. 2010;68.

35. Razeghi P, Baskin KK, Sharma S, Young ME, Stepkowski S, Essop MF, et al. Atrophy, hypertrophy, and hypoxemia induce transcriptional regulators of the ubiquitin proteasome system in the rat heart. Biochemical and biophysical research communications. 2006;342:361-4.

36. Li H, Malhotra S, Kumar A. Nuclear factor-kappa B signaling in skeletal muscle atrophy. J Mol Med. 2008;86:1113-26.

37. Stitt TN, Drujan D, Clarke Ba, Panaro F, Timofeyva Y, Kline WO, et al. The IGF-1/PI3K/Akt Pathway Prevents Expression of Muscle Atrophy-Induced Ubiquitin Ligases by Inhibiting FOXO Transcription Factors. Mol Cell. 2004;14:395-403.

38. Yoshida T, Semprun-Prieto L, Sukhanov S, Delafontaine P. IGF-1 prevents ANG II-induced skeletal muscle atrophy via Akt- and Foxo-dependent inhibition of the ubiquitin ligase atrogin-1 expression. American journal of physiology Heart and circulatory physiology. 2010;298:H156570.

39. Sartori R, Milan G, Patron M, Mammucari C, Blaauw B, Abraham R, et al. Smad2 and 3 transcription factors control muscle mass in adulthood. American journal of physiology Cell physiology. 2009;296:C1248-57.

40. Trendelenburg AU, Meyer A, Rohner D, Boyle J, Hatakeyama S, Glass DJ. Myostatin reduces Akt/TORC1/p70S6K signaling, inhibiting myoblast differentiation and myotube size. American journal of physiology Cell physiology. 2009;296:C1258-70.

41. Lundby C, Calbet JaL, Robach P. The response of human skeletal muscle tissue to hypoxia. Cellular and molecular life sciences : CMLS. 2009;66:3615-23.

42. Flueck M. Plasticity of the muscle proteome to exercise at altitude. High altitude medicine \& biology. 2009;10(2):183-93. 
43. Murray AJ. Metabolic adaptation of skeletal muscle to high altitude hypoxia: how new technologies could resolve the controversies. Genome medicine. 2009;1(12):117.

44. Gosker HR, Schols AM. Muscle metabolic modulation by chronic hypoxia. Journal of proteome research. 2007;6(9):3400-1.

45. Vijayasarathy C, Damle S, Prabu SK, Otto CM, Avadhani NG. Adaptive changes in the expression of nuclear and mitochondrial encoded subunits of cytochrome c oxidase and the catalytic activity during hypoxia. Eur J Biochem. 2003;270(5):871-9.

46. Formenti F, Constantin-Teodosiu D, Emmanuel Y, Cheeseman J, Dorrington KL, Edwards LM, et al. Regulation of human metabolism by hypoxia-inducible factor. Proc Natl Acad Sci U S A. 2010;107(28):12722-7.

47. Mason SD, Howlett RA, Kim MJ, Olfert IM, Hogan MC, McNulty W, et al. Loss of skeletal muscle HIF-1alpha results in altered exercise endurance. PLoS biology. 2004;2(10):e288.

48. Regnault TR, Zhao L, Chiu JS, Gottheil SK, Foran A, Yee SP. Peroxisome Proliferator-Activated Receptor -beta/delta, -gamma Agonists and Resveratrol Modulate Hypoxia Induced Changes in Nuclear Receptor Activators of Muscle Oxidative Metabolism. PPAR research. 2010;2010:129173.

49. Lunde IG, Anton SL, Bruusgaard JC, Rana ZA, Ellefsen S, Gundersen K. Hypoxia inducible factor 1 links fast-patterned muscle activity and fast muscle phenotype in rats. The Journal of physiology. 2011;589(Pt 6):1443-54.

50. Mounier R, Pedersen BK, Plomgaard P. Muscle-specific expression of hypoxia-inducible factor in human skeletal muscle. Experimental physiology. 2010;95(8):899-907.

51. Remels AH, Gosker HR, Schrauwen P, Langen RC, Schols AM. Peroxisome proliferator-activated receptors: a therapeutic target in COPD? The European respiratory journal. 2008;31(3):5028.

52. Remels AH, Langen RC, Schrauwen P, Schaart G, Schols AM, Gosker HR. Regulation of mitochondrial biogenesis during myogenesis. Molecular and cellular endocrinology. 2010;315(12):113-20.

53. Shoag J, Arany Z. Regulation of hypoxia-inducible genes by PGC-1 alpha. Arteriosclerosis, thrombosis, and vascular biology. 2010;30(4):662-6.

54. O'Hagan KA, Cocchiglia S, Zhdanov AV, Tambuwala MM, Cummins EP, Monfared M, et al. PGC-1alpha is coupled to HIF-1alpha-dependent gene expression by increasing mitochondrial oxygen consumption in skeletal muscle cells. Proc Natl Acad Sci U S A. 2009;106(7):2188-93.

55. Hayden MS, Ghosh S. Shared principles in NF-kappaB signaling. Cell. 2008;132(3):344-62.

56. Remels AH, Gosker HR, Schrauwen P, Hommelberg PP, Sliwinski P, Polkey M, et al. TNFalpha impairs regulation of muscle oxidative phenotype: implications for cachexia? FASEB journal : official publication of the Federation of American Societies for Experimental Biology. 2010;24(12):5052-62.

57. Mendias CL, Marcin JE, Calerdon DR, Faulkner JA. Contractile properties of EDL and soleus muscles of myostatin-deficient mice. J Appl Physiol 2006;101(3):898-905.

58. Macpherson PC, Wang X, Goldman D. Myogenin regulates denervation-dependent muscle atrophy in mouse soleus muscle. J Cell Biochem. 2011;112(8):2149-59. 
59. Sato S, Suzuki H, Tsujimoto H, Shirato K, Tachiyashiki K, Imaizumi K. Casted-immobilization downregulates glucocorticoid receptor expression in rat slow-twitch soleus muscle. Life Sci. 2011;89(25-26):962-7.

60. Itoh K, Moritani T, Ishida K, Hirofuji C, Taguchi S, Itoh M. Hypoxia-induced fibre type transformation in rat hindlimb muscles. Histochemical and electro-mechanical changes. Eur J Appl Physiol Occup Physiol. 1990;60(5):331-6.

61. Eltzschig HK, Carmeliet P. Hypoxia and inflammation. The New England journal of medicine. 2011;364(7):656-65.

62. Taylor CT, Cummins EP. The role of NF-kappaB in hypoxia-induced gene expression. Annals of the New York Academy of Sciences. 2009;1177:178-84.

63. Remels $A H$, Schrauwen P, Broekhuizen R, Willems J, Kersten S, Gosker HR, et al. Peroxisome proliferator-activated receptor expression is reduced in skeletal muscle in COPD. The European respiratory journal. 2007;30(2):245-52.

64. Doucet M, Russell AP, Léger B, Debigaré R, Joanisse DR, Caron M-A, et al. Muscle atrophy and hypertrophy signaling in patients with chronic obstructive pulmonary disease. American journal of respiratory and critical care medicine. 2007;176:261-9.

65. Plant PJ, Brooks D, Faughnan M, Bayley T, Bain J, Singer L, et al. Cellular markers of muscle atrophy in chronic obstructive pulmonary disease. American journal of respiratory cell and molecular biology. 2010;42:461-71.

66. Crul T, Testelmans D, Spruit MA, Troosters T, Gosselink R, Geeraerts I, et al. Gene expression profiling in vastus lateralis muscle during an acute exacerbation of COPD. Cellular physiology and biochemistry : international journal of experimental cellular physiology, biochemistry, and pharmacology. 2010;25(4-5):491-500.

67. Jatta K, Eliason G, Portela-Gomes GM, Grimelius L, Caro O, Nilholm L, et al. Overexpression of von Hippel-Lindau protein in skeletal muscles of patients with chronic obstructive pulmonary disease. Journal of clinical pathology. 2009;62:70-6.

68. Agusti A, Morla M, Sauleda J, Saus C, Busquets X. NF-kappaB activation and iNOS upregulation in skeletal muscle of patients with COPD and low body weight. Thorax. 2004;59(6):483-7.

69. Takabatake N, Nakamura H, Abe S, Inoue S, Hino T, Saito H, et al. The relationship between chronic hypoxemia and activation of the tumor necrosis factor-alpha system in patients with chronic obstructive pulmonary disease. American journal of respiratory and critical care medicine. 2000;161(4 Pt 1):1179-84.

70. Taylor CT. Interdependent roles for hypoxia inducible factor and nuclear factor-kappaB in hypoxic inflammation. The Journal of physiology. 2008;586(Pt 17):4055-9.

71. McLoughlin DM, Spargo E, Wassif WS, Newham DJ, Peters TJ, Lantos PL, et al. Structural and functional changes in skeletal muscle in anorexia nervosa. Acta neuropathologica. 1998;95(6):632-40.

72. Sandri M. Signaling in muscle atrophy and hypertrophy. Physiology. 2008;23:160-70.

73. Cong H, Sun L, Liu C, Tien P. Inhibition of atrogin-1/MAFbx expression by adenovirus-delivered small hairpin RNAs attenuates muscle atrophy in fasting mice. Human gene therapy. $2011 ; 22(3): 313-24$. 
74. Jagoe RT, Lecker SH, Gomes M, Goldberg AL. Patterns of gene expression in atrophying skeletal muscles: response to food deprivation. FASEB journal : official publication of the Federation of American Societies for Experimental Biology. 2002;16(13):1697-712.

75. Wing SS, Goldberg AL. Glucocorticoids activate the ATP-ubiquitin-dependent proteolytic system in skeletal muscle during fasting. The American journal of physiology. 1993;264(4 Pt 1):E668-76.

76. Takabatake N, Nakamura H, Minamihaba O, Inage M, Inoue S, Kagaya S, et al. A novel pathophysiologic phenomenon in cachexic patients with chronic obstructive pulmonary disease: the relationship between the circadian rhythm of circulating leptin and the very low-frequency component of heart rate variability. American journal of respiratory and critical care medicine. 2001;163(6):1314-9.

77. Creutzberg EC, Wouters EF, Vanderhoven-Augustin IM, Dentener MA, Schols AM. Disturbances in leptin metabolism are related to energy imbalance during acute exacerbations of chronic obstructive pulmonary disease. American journal of respiratory and critical care medicine. 2000;162(4 Pt 1):1239-45.

78. Gardner DS, Fletcher AJ, Fowden AL, Giussani DA. Plasma adrenocorticotropin and cortisol concentrations during acute hypoxemia after a reversible period of adverse intrauterine conditions in the ovine fetus during late gestation. Endocrinology. 2001;142(2):589-98.

79. Kodama T, Shimizu N, Yoshikawa N, Makino Y, Ouchida R, Okamoto K, et al. Role of the glucocorticoid receptor for regulation of hypoxia-dependent gene expression. J Biol Chem. 2003;278(35):33384-91.

80. Moncloa F, Donayre J, Sobrevilla LA, Guerra-Garcia R. Endocrine studies at high altitude. II. Adrenal cortical function in sea level natives exposed to high altitudes (4300 metersfor two weeks. The Journal of clinical endocrinology and metabolism. 1965;25(12):1640-2.

81. Larsen JJ, Hansen JM, Olsen NV, Galbo H, Dela F. The effect of altitude hypoxia on glucose homeostasis in men. The Journal of physiology. 1997;504 ( Pt 1):241-9.

82. Leonard MO, Godson C, Brady HR, Taylor CT. Potentiation of glucocorticoid activity in hypoxia through induction of the glucocorticoid receptor. Journal of immunology. 2005;174(4):2250-7.

83. Shavlakadze T, Anwari T, Soffe Z, Cozens G, Mark PJ, Gondro C, et al. Impact of fasting on the rhythmic expression of myogenic and metabolic factors in skeletal muscle of adult mice. American journal of physiology Cell physiology. 2013;305(1):C26-35.

84. Stahn C, Buttgereit F. Genomic and nongenomic effects of glucocorticoids. Nature clinical practice Rheumatology. 2008;4(10):525-33.

85. Sandri M, Sandri C, Gilbert A, Skurk C, Calabria E, Picard A, et al. Foxo transcription factors induce the atrophy-related ubiquitin ligase atrogin-1 and cause skeletal muscle atrophy. Cell. 2004;117:399-412.

86. McLoughlin TJ, Smith SM, DeLong AD, Wang H, Unterman TG, Esser KA. FoxO1 induces apoptosis in skeletal myotubes in a DNA-binding-dependent manner. American journal of physiology Cell physiology. 2009;297(3):C548-55. 
87. Shimizu N, Yoshikawa N, Ito N, Maruyama T, Suzuki Y, Takeda S-i, et al. Crosstalk between glucocorticoid receptor and nutritional sensor mTOR in skeletal muscle. Cell metabolism. 2011;13:170-82.

88. Wang H, Kubica N, Ellisen LW, Jefferson LS, Kimball SR. Dexamethasone represses signaling through the mammalian target of rapamycin in muscle cells by enhancing expression of REDD1. J Biol Chem. 2006;281(51):39128-34.

89. Hu Z, Wang H, Lee IH, Du J, Mitch WE. Endogenous glucocorticoids and impaired insulin signaling are both required to stimulate muscle wasting under pathophysiological conditions in mice. The Journal of clinical investigation. 2009;119(10):3059-69.

90. Medina R, Wing SS, Goldberg AL. Increase in levels of polyubiquitin and proteasome mRNA in skeletal muscle during starvation and denervation atrophy. Biochem J. 1995;307 ( Pt 3):631-7. 


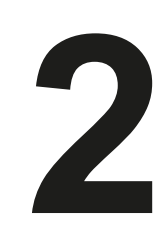

\section{Distinct responses of protein turnover- regulatory pathways in hypoxia- and semi-starvation induced muscle atrophy}

C.C. de Theije, R.J.C. Langen, W.H. Lamers, A.M.W.J. Schols, S.E. Köhler

Am J Physiol Lung Cell Mol Physiol. 2013 Jul 1;305(1):L82-91 


\section{Abstract}

The balance of muscle protein synthesis and degradation determines skeletal muscle mass. We hypothesized that hypoxia-induced muscle atrophy and alterations in the regulation of muscle protein turnover include a hypoxia-specific component, in addition to the observed effects of reduction in food intake in response to hypoxia. Mice were subjected to normoxic, hypoxic ( $8 \%$ oxygen) or pair-fed conditions for 2, 4 and 21 days. Cell-autonomous effects of hypoxia on skeletal muscle were also assessed in differentiated $\mathrm{C} 2 \mathrm{C} 12$ myotubes. Hypoxia induced an initial rapid loss of body and muscle weight, which remained decreased during chronic hypoxia, and could only in part be explained by the hypoxia-induced reduction of food intake (semi-starvation). Regulatory steps of protein synthesis (unfolded protein response and mTOR signaling) remained active in response to acute and sustained hypoxia but not to semi-starvation. Activation of regulatory signals for protein degradation, including increased expression of Murf1, Atrogin-1, Bnip3 and Map1/c3b mRNAs, was observed in response to acute hypoxia, and to a lesser extent following semi-starvation. Conversely, the sustained elevation of Atrogin-1, Bnip3 and Map1/c3b mRNAs and the increased activity of their upstream transcriptional regulator FOXO1 were specific to chronic hypoxia, because they were not observed in response to reduced food intake. In conclusion, altered regulation of protein turnover during hypoxia-induced muscle atrophy resulted from an interaction of semi-starvation and a hypoxia-specific component. The finding that food restriction but not hypoxia-induced semi-starvation inhibited regulatory steps in protein synthesis suggests a hypoxia-specific impairment of the coordination between protein -synthesis and-degradation signaling in skeletal muscle. 


\section{Introduction}

Weight loss and muscle atrophy are common features in advanced chronic obstructive pulmonary disease (COPD). In addition to nutritional imbalance, systemic inflammation and progressive inactivity, hypoxemia has been implicated as trigger for muscle atrophy in COPD patients. Hypoxemia is a symptom during the whole course of the disease with chronic hypoxemia in end-stage COPD and acute hypoxemic episodes during exacerbations [1]. Understanding the mechanisms by which hypoxia induces muscle atrophy will benefit the treatment of this disease, since loss of muscle mass is a strong predictor of mortality and significantly increases disease burden [2-6]. Although exposure to hypobaric hypoxia at high-altitudes results in muscle atrophy in men and animals [7-10], it is still under debate whether hypobaric hypoxia induces other physiological effects than normobaric hypoxia [11, 12]. It is not clear whether findings from hypobaric hypoxia studies related to skeletal muscle loss can be translated directly to the situation of COPD patients who experience normobaric hypoxia and also show muscle atrophy. We therefore have chosen a normobaric hypoxia mouse model to investigate the effects of hypoxia on skeletal muscle mass. Hypoxemia and chronic respiratory failure have also been associated with decreased appetite and malnutrition [13-15]. The contribution of lowered energy intake to hypoxia-induced muscle atrophy has been investigated at high altitude [16], but the effects of normobaric hypoxia on energy intake and signaling pathways regulating protein turnover in skeletal muscle have not been studied earlier to our knowledge. Protein turnover is the balance between protein synthesis and protein degradation. Key regulatory elements of protein synthesis are the mammalian target of rapamycin complex 1 (mTORC1) [17] and endoplasmic reticulum (ER)-stress signaling [18]. Activation of mTORC1 signaling stimulates protein synthesis through phosphorylation of eukaryotic translation initiation factor 4E binding protein 1 (EIF4EBP1, a.k.a. 4E$\mathrm{BP} 1$ ) at threonine (Thr) residues 37 and 46. This initial phosphorylation primes 4E-BP1 for further phosphorylation at serine (Ser) 65 and Thr70, whereupon it dissociates from EIF4E thereby making it available for mRNA translation [19, 20]. In addition, mTORC1 can regulate mRNA translation through activation of ribosomal protein S6 kinase $\beta 1$ (P70S6K1) at phosphorylation site Thr389. This initial phosphorylation is required to allow full activation of the kinase by an additional phosphorylation at Thr229 catalyzed by phosphoinositide-dependent kinase 1[21]. ER-stress inhibits general protein synthesis through the phosphorylation of eukaryotic translation initiation factor $2 \alpha$ (EIF2 $\alpha$ ) by the unfolded protein response (UPR) at phosphorylation site Ser51. Phosphorylation of EIF2 $\alpha$ reduces but does not completely shut down global protein synthesis and translation of selected mRNAs may even be increased [22, 23]. Proteasomal and lysosomal protein degradation play important roles in muscle catabolism. Tripartite motif containing 
63, E3 ubiquitin protein ligase (TRIM63, a.k.a. MURF-1) and F-box protein 32 (FBXO32, a.k.a. ATROGIN-1) are rate-determining enzymes of the proteasomal protein degradation machinery in skeletal muscle. Increased mRNA expression of these genes can be used as marker for the activation of these pathways [24, 25]. Furthermore, these genes are positively regulated by the transcription factor Forkhead box $\mathrm{O} 1$ (FOXO1) [26-28]. Increased expression of BCL2/adenovirus E1B 19kDa protein interacting protein 3 (BNIP3) and microtubule-associated protein 1 light chain-3B (MAP1LC3B) has been used to demonstrate activation of lysosomal protein degradation [29, 30]. The expression of the respective genes, Bnip3 and Map1/c3b, is also subject to regulation by the UPR and hypoxia-inducible factor 1a (HIF1a) [31, 32] Protein kinase B (PKB, a.k.a. AKT) functions as a central regulator of both protein synthesis and degradation. AKT is activated through the phosphorylation of Thr308 by 3-phosphoinositide-dependent protein kinase 1 (PDK1), which then facilitates phosphorylation of Ser473 by a complex of proteins containing mammalian target of rapamycin complex 2 (mTORC2) [33]. Activated AKT can inhibit FOXO1 activity and subsequent transcription of markers of proteasomal protein degradation, such as Murf1 and Atrogin-1 [34]. Furthermore, activated AKT can activate mTORC1 and thus stimulate mRNA translation through inactivation of the inhibitors tuberous sclerosis complex 1 and 2 (TSC1/TSC2). COPD patients may experience in addition to chronic mild hypoxia, episodes of acute severe hypoxia during exacerbations. Both mild and severe episodes of hypoxia may contribute to the muscle atrophy observed in these patients. In order to model the adaptive effects of long-term chronic hypoxia and the activation of signaling pathways during acute severe episodes within a feasible experimental time range, we chose severe hypoxic conditions for 21 days in our mouse model. This allowed us to show both the activation of relevant pathways in the acute progressive phase (0-4 days) and long-term adaptive consequences (21 days). Control animals breathed ambient air, whereas for the hypoxic animals oxygen was partially replaced by N2. To distinguish the contribution of reduced food intake on the decrease of skeletal muscle mass and protein turnover signaling from hypoxia-specific effects, a normoxic group pair-fed to the hypoxic group was included. To determine to what extent the hypoxia-specific effects were due to direct effects on skeletal muscle, that is, cell-autonomous effects or the consequence of systemic effects, mRNA expression levels of target genes were also measured in hypoxia-exposed C2C12 myotubes.

We have measured the expression of relevant genes and the activation status of these key proteins of several pathways involved in protein synthesis and degradation to assess the effect of hypoxia on the activity of protein turnover signaling pathways. We hypothesize that normobaric hypoxia induces muscle atrophy, which is not completely attributable to semi-starvation. Furthermore hypoxia- 
induced muscle atrophy involves a coordinated increase in muscle proteolysis signaling and a decrease of regulatory signals for protein synthesis.

\section{Methods}

\section{Animals and tissue collection.}

Twelve-week-old C57BL/6J male mice (Charles River Laboratories international, Wilmington, MA, USA) ( $n=72$ ) were randomly divided into 3 groups: normoxia $(\mathrm{N})$, normoxia pair-fed to hypoxic animals (PF), and normobaric hypoxia $(\mathrm{H})$. All mice were housed in experimental chambers at $21^{\circ} \mathrm{C}$ with a 12-hour dark/light cycle. Mice received standard chow (V1534-000 Ssniff R/M-H, Ssniff Spezialdiäten, $\mathrm{GmbH}$, Soest, Germany) and water ad libitum. After 5 days of acclimatization, 48 mice were exposed to $21 \% \mathrm{O} 2$ (N-group) and 24 to normobaric hypoxia $(\mathrm{H}$ group). Using the proOX system P110 (BioSpherix, Lacona, NY, USA), oxygen levels were reduced in a stepwise manner to $12 \%$ (day 1 ), $10 \%$ (day 2), and finally $8 \%(61 \mathrm{mmHg})$ on day 3 , which was then maintained for the remainder of the experiment. To control for the effects of reduced food intake during hypoxia, 24 mice of the normoxia group received the amount of food consumed by the hypoxic mice (PF-group). 8 mice per condition were sacrificed on days 2, 4 and 21. Abdominal aortic blood was collected under general anesthesia with isoflurane (IsoFlo, Abbott Laboratories, Illinois, U.S.A) at an oxygen pressure corresponding to the respective experimental condition. Tissues were isolated and either frozen immediately in liquid nitrogen, fixed in formalin or frozen in Tissue-Tek (Sakura Finetek Europe B.V., Zoeterwoude, NL) for further analysis. Tissue weights were corrected for bodyweight at the start of the experiment. All experimental procedures were approved by the Committee for Animal Care and Use of Maastricht University.

\section{Blood parameters.}

Abdominal aortic blood was collected into a 1-mL syringe (Becton Dickinson B.V., Breda, NL) coated with heparin (Leo Pharma B.V., Breda, NL). Oxygen levels and $\mathrm{pH}$ were measured immediately using the ABL 510 Blood Gas Analyzer (Radiometer Benelux B.V., Zoetermeer, NL) and blood cell count was determined with the Coulter Ac T Diff hematology Analyzer (Beckman Coulter GmbH, Krefeld, DE).

\section{Western blotting.}

Tissue was ground to powder using an N2-cooled steel mortar. $50 \mathrm{mg}$ powder was lysed in $1 \mathrm{~mL}$ lysis buffer (10 mM Tris, $\mathrm{pH} 7.4 ; 100 \mathrm{mM} \mathrm{NaCl} ; 1 \mathrm{mM}$ EDTA, 1 mM EGTA; 1mM NaF; 20 mM Na4P2O7; 2 mM activated Na3VO4; $0.1 \%$ SDS, $1 \%$ sodium deoxycholate; $1 \%$ triton $\mathrm{X}-100 ; 10 \%$ glycerol; $17.5 \mathrm{mM}$ $\beta$-glycerophosphate (all chemicals from Sigma-Aldrich Chemie B.V, Zwijndrecht, 
$\mathrm{NL}$ ); and protease inhibitors (Complete, Roche Nederland B.V., Woerden, NL)). Crude lysates were incubated on ice for $30 \mathrm{~min}$., followed by $10 \mathrm{~min}$. centrifugation at $16,000 \mathrm{~g}$. Total protein concentration of the supernatant was determined with a bicinchoninic acid (BCA) protein assay kit (Pierce Biotechnology Inc, Rockford, USA) according to manufacturer's instructions. Samples were analyzed by Western blot. Briefly, $12.5 \mu \mathrm{g}$ of protein per lane were separated on a Criterion $^{\mathrm{TM}}$ XT Precast 4-12\% Bis-Tris gel (Bio-Rad Laboratories B.V., Veenendaal, $\mathrm{NL}$ ), and transferred to a nitrocellulose transfer membrane (Bio-Rad Laboratories B.V., Veenendaal, NL) by electroblotting. The membrane was stained with Ponceau $S$ solution $(0.2 \%$ Ponceau $S$ in $1 \%$ acetic acid; Sigma-Aldrich Chemie B.V, Zwijndrecht, NL) to control for protein loading. The membrane was blocked for $1 \mathrm{hr}$ at room temperature in $5 \%(\mathrm{w} / \mathrm{v})$ non-fat dried milk (ELK, Campina B.V., Zaltbommel, NL) dissolved in TBS-Tween-20 (0.05\%). Nitrocellulose blots were washed in TBS-Tween-20 $(0.05 \%)$ on a rocking platform for $5 \mathrm{~min}$; followed by overnight incubation at $4{ }^{\circ} \mathrm{C}$ with primary antibodies (EIF2a: \#9722, p-EIF2a(Ser51): \#3398, AKT: \#4685, p-AKT(Ser473): \#9271, P70S6K1: \#9202, p-P70S6K1(Thr389): \#9205, 4E-BP1: \#9452, p-4E-BP1(Ser65): \#9451, FOXO1: \#2880, p-FOXO1(Ser256): \#9461, GSK3- 3 : \#9315, p-GSK3- $\beta(S e r 9): \# 9336)$ (Cell Signaling Technology, Beverly, MA, USA). All antisera were diluted 1/1,000 in TBS-Tween-20 (0.05\%). After three washing steps of $10 \mathrm{~min}$ each, blots were probed with a horseradish peroxidase-conjugated secondary antibody (Vector Laboratories, Burlingame, CA, USA) and visualized with chemiluminescence (Supersignal West Pico or Femto Chemiluminescent Substrate; Pierce Biotechnology Inc, Rockford, USA) in a LAS-3000 Luminescent Image analyzer (Fujifilm, Tokyo, Japan). Bands were quantified using the AIDA software (Fujifilm, Tokyo, Japan). All data were corrected for protein loading as determined after Ponceau $S$ staining.

\section{Cell culture.}

The murine skeletal muscle cell line C2C12 (\#CRL1772, ATCC, LGC Standards, Molsheim Cedex, FR) was used for all in vitro experiments. In addition, a stably transfected $\mathrm{C} 2 \mathrm{C} 12$ cell-line was generated which carries a luciferase reporter plasmid (pGL3) with the hypoxia responsive element (HRE)[35]. Cells were cultured in growth medium (GM) consisting of Dulbecco's modified Eagle's medium (DMEM) supplemented with $1,000 \mathrm{mg} / \mathrm{L}$ glucose, antibiotics (50 Units/ml penicillin and $50 \mu \mathrm{g} / \mathrm{mL}$ streptomycin) and $10 \%(\mathrm{v} / \mathrm{v}$ ) fetal bovine serum (FBS) (all from Gibco, Rockville, MD, USA). C2C12 cells were plated and cultured in GM for 24 hrs. To induce differentiation, GM was replaced with differentiation medium (DM: $1,000 \mathrm{mg} / \mathrm{L}$ glucose, $1.0 \%$ heat-inactivated FBS and antibiotics in DMEM). After 5 days of differentiation, cells were exposed to either normoxic (normal) or hypoxic 
(4\% oxygen) conditions for 24 hrs. Cells were washed with PBS and RNA was isolated using the RNeasy mini kit (QIAGEN Benelux B.V., Venlo, NL).

\section{Luciferase measurement.}

Cells were harvested in 1x Reporter Lysis Buffer (Promega, Madison, WI, USA) and incubated on ice for 10 minutes Cell lysates were centrifuged (14000 rpm, 1 min) and supernatants were snap frozen and stored at $-80{ }^{\circ} \mathrm{C}$ for later analysis. Luciferase activity was measured according to manufacturer's instructions (Promega) and corrected for total protein content (Pierce Biotechnology Inc, Rockford, USA).

\section{RT-qPCR.}

Total RNA was isolated using the TRI REAGENT TM (Sigma-Aldrich Chemie B.V, Zwijndrecht, NL) and further purified by precipitation with $2 \mathrm{M} \mathrm{LiCl}$. cDNA synthesis was performed with random hexamer primers on denatured RNA using the Transcriptor First Strand cDNA Synthesis Kit (Roche Nederland B.V., Woerden, $\mathrm{NL}$ ). Real-time quantitative PCR was performed in the iQ5 thermal cycler (Biorad, Hercules, CA, USA) using the qPCR SyBr Green Fluorescein Mix (Abgene, Epsom, UK). qPCR primers were designed using Primer Express 2.0 software (Applied Biosystems, Carlsbad, CA, USA) and ordered from Sigma Genosys (Table 1). All primers were intron-spanning to avoid contamination of the amplification products with genomic DNA. Expression of genes was normalized to $18 \mathrm{~S}$ ribosomal RNA.

\begin{tabular}{llll}
\hline \hline gene & NCBI & Forward primer (5'-3') & Reverse primer (5'-3') \\
\hline 18S & NR_003278.1 & AGTTAGCATGCCAGAGTCTCG & TGCATGGCCGTTCTTAGTTG \\
Ca-9 & NM_139305.2 & CAGGAGGCCTGGCAGTTTT & TTCTTCCAAATGGGACAGCAA \\
Glut-1 & NM_011400.3 & TGACCATCGCCCTGGCCT & GGACCAGGGCCTACTTCAAAG \\
Atf4 & NM_009716.2 & GGAATGGCCGGCTATGG & CATTTCTCCAACATCCAATCTGT \\
Gadd34 & NM_008654 & GGCGGCTCAGATTGTTCAAA & AAGGAAATGGACTGTGACTTTCTCA \\
Foxo1 & NM_019739.3 & AAGAGCGTGCCCTACTTCAAGGATA & CCATGGACGCAGCTCTTCTC \\
Murf1 & NM_00103904 & TGTCTGGAGGTCGTTTCCG & CTCGTCTTCGTGTTCCTTGC \\
Atrogin-1 & NM_026346.2 & ACCGGCTACTGTGGAAGAGA & CCTTCCAGGAGAGAATGTGG \\
Bnip3 & NM_009760.4 & CCATGTCGCAGAGCGGG & GACGGAGGCTGGAACGC \\
Mapllc3b & NM_026160.4 & GAGCAGCACCCCACCAAGAT & CGTGGTCAGGCACCAGGAA \\
\hline
\end{tabular}

Table 1. RT-qPCR primer sequences

\section{Statistical analysis.}

Data are shown as means \pm SEM. Comparisons were computed with SPSS version 15 (SPSS Inc., IL, USA). Statistical significance was tested for in vivo data by ANOVA. The type of post-hoc analysis was based on data variance, with the Tukey post-hoc test for data with equal variance, Games-Howell post-hoc test for all other cases. For in vitro data Student's t-test was used. A P-value $<0.05$ was considered statistically significant and a $P$-value $0.05 \leq P \leq 0.1$ as indicating a 
trend. In the Figures, P-values for comparisons of the different groups $(\mathrm{H}, \mathrm{N}$ and PF) are shown.

\section{Results}

\section{Hypoxia induces hypoxemia, acidosis and polycythemia.}

Partial pressure of oxygen in blood ( $\mathrm{PaO} 2), \mathrm{pH}$, oxygen saturation of hemoglobin $(\mathrm{SaO} 2)$ and red blood-cell count were determined in blood collected from the abdominal aorta. $\mathrm{PaO} 2$ of the normoxic and pair-fed groups remained around $125-140 \mathrm{mmHg}$, whereas under hypoxic conditions $\mathrm{PaO} 2$ was decreased to 54 $\mathrm{mmHg}$ after day 2 and to $33 \mathrm{mmHg}$ on day 21 (PaSO2 d21 vs d2 P<0.01, d21 vs $\mathrm{d} 4 \mathrm{P}<0.01$ ) (Figure. $1 \mathrm{~A}$ ). Hypoxia also lowered the blood $\mathrm{pH}$ by 0.2 units as expected [37] (Figure. 1B). Reductions of $\mathrm{PaO} 2$ and $\mathrm{pH}$ affect $\mathrm{SaO} 2$, which accordingly decreased to $\sim 57 \%$ within the first 2 days. After 21 days, $\mathrm{SaO} 2$ was $\sim 24 \%$ of the normoxic value in hypoxic animals $(\mathrm{SaO} 2 \mathrm{~d} 21$ vs d2 $\mathrm{P}<0.01$, d21 vs d4 $P<0.05$ ) (Figure 1C). An increase of red blood cells (RBC) to $\sim 150 \%$ of control was observed in the hypoxic group after 21 days (RBC d21 vs d2 $P<0.01$, d21 vs d4 $P<0.01$ ) (Figure 1D). Reduced food intake alone had no or only minor effects on $\mathrm{PaO} 2, \mathrm{pH}, \mathrm{SaO} 2$ and red blood cell count.

\section{Hypoxia induces differential expression of hypoxia-sensitive genes in skeletal muscle.}

To assess tissue hypoxia in muscle, we measured the expression of two welldescribed HIF-1 $\alpha$ target genes, carbonic anhydrase IX (Ca-9) and glucose transporter 1 (Glut1) [38, 39]. Compared to normoxia, Glut-1 expression in gastrocnemius muscle increased transiently in response to hypoxia (Figure $1 \mathrm{E}$ ), whereas Ca-9 expression was upregulated only at day 21, implying that Glut-1 and Ca-9 are parameters for subacute and chronic hypoxia (Figure 1F). Food restriction also induced a transient increase in Glut-1 expression at day 2, which was decreased at day 21. Semi-starvation could not account for hypoxia-induced $\mathrm{Ca}-9$ expression. Partial oxygen pressure in capillaries delivering oxygen to skeletal muscle is much lower than that in inhaled air or arterial blood [40]. Extrapolating from these data, the partial oxygen pressure in capillaries in animals exposed to $8 \% \mathrm{O} 2$ is 3 to $5 \%$. We therefore chose $4 \%$ ambient oxygen in the cell culture experiments. Under these conditions, only $3 \%$ of the myotubes were damaged after $24 \mathrm{hrs}$, whereas exposure to low concentrations of hydrogenperoxide for the same time induced $14 \%$ damage, as expected [36]. Under normoxic conditions, $1 \%$ of the myotubes incurred cell damage. Exposure of $\mathrm{C} 2 \mathrm{C} 12$ cells to $4 \%$ oxygen resulted in a clear induction of HIF-1 $\alpha$ activity (Figure 1G). The expression of the HIF1a-regulated genes Ca-9 and Glut1 was increased 140- and 6-fold, respectively, within 24 hours (Figure $1 \mathrm{H}$ ). 


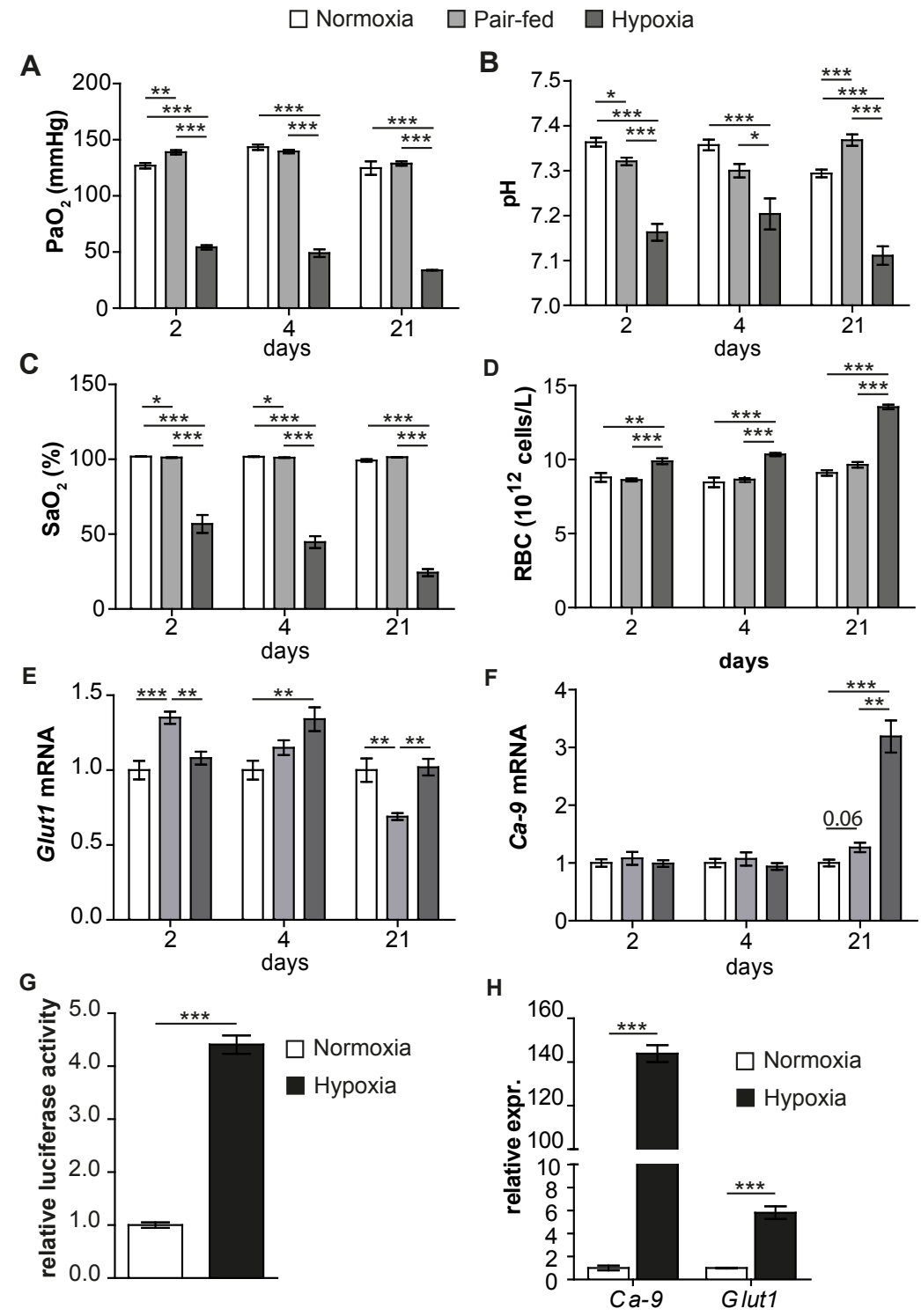

Figure 1. Hypoxia induces hypoxemia, acidosis, polycythemia and differential expression of hypoxia-sensitive genes in skeletal muscle. Mice ( $n=6-8$ per group) were exposed to normoxia or hypoxia or were pair-fed to hypoxic mice for 21 days. Blood was sampled from the abdominal aorta at the indicated time points and the following parameters were determined: $(A) \mathrm{PaO} 2$, (B) $\mathrm{pH},(C) \mathrm{SaO}$, (D) RBC concentration. mRNA expression of marker genes was measured in the gastrocnemius muscle: (E) Glut1, (F) Ca-9. (G) Luciferase expression in C2C12 cells, stably transfected with a hypoxiaresponsive element reporter plasmid $(n=6)$ and cultured at an ambient oxygen concentration of $4 \%$. (H) Expression of $\mathrm{Ca}-9$ and Glut1 in $\mathrm{C} 2 \mathrm{C} 12$ myotubes exposed to an ambient oxygen concentration of $4 \%(n=3)$. Relative expression of mRNAs was corrected for $18 S$ RNA concentration and normalized to normoxic values of the corresponding time points. Significant differences between groups at a given time point are indicated by asterisks, with ${ }^{*}=P \leq 0.05,{ }^{* *}=P \leq 0.01$ or ${ }^{* * *}=P \leq 0.001$. Trends are indicated by the specific $P$-value. ( $n=6-8$ per group). 
Overall, experimental mice experienced severe hypoxemia as demonstrated by the adaptations of blood values, such as decreased partial oxygen pressure, oxygen saturation and $\mathrm{pH}$, and the compensatory increase of red blood cells. In addition, our data indicated that the initial response to hypoxia differs from the long-term adaptive response, as illustrated by the differential expression of hypoxia-sensitive genes.

\section{Decreases in body-, muscle- and adipose weight following hypoxia indicate} a hypoxia-specific component independent of food intake.

Exposure to hypoxia caused a pronounced reduction in food intake (semi-starvation). In comparison with the normoxic control group (N-group), food intake of mice exposed to normobaric hypoxia (H-group) decreased to $~ 50 \%$ on day 4 and gradually recovered to $\sim 80 \%$ of the control intake on day 10 to remain constant thereafter (Figure 2A). To distinguish specific effects of hypoxia from those mediated by the reduced food intake, a normoxic pair-fed group (PF-group) was included that received the same amount of food as consumed by the H-group. Mice in the PF-group lost less body weight during the first 4 days ( $\sim 91 \%$ of $\mathrm{N}$-group) than mice exposed to hypoxia ( $\sim 85 \%$ of $\mathrm{N}$-group) and continued to lose weight until day 7 (Figure 2B). Normoxic mice gained little weight throughout the experiment ( $0.1 \mathrm{gram} /$ week), whereas pair-fed and hypoxic mice showed a higher, but comparable weight gain ( $\sim 0.5 \mathrm{gram} /$ week) after the initial weight loss. After 21 days, both pair-fed and hypoxic mice still had lower body weights than normoxic animals ( $\sim 96 \%$ and $\sim 90 \%$ for the PF- and H-group, respectively). To assess the contribution of adipose tissue to the observed loss in body weight, we determined the weight of the epididymal fat pad (Figure 2C). Semi-starvation alone did not affect fat pad weight, whereas hypoxia caused a significant reduction, which developed most markedly during the first 4 days ( $71 \%$ of $\mathrm{N}$-group), but continued thereafter, resulting in a loss of $\sim 50 \%$ of $\mathrm{N}$-group on day 21 (d21 vs d2 $\mathrm{P}<0.01$ ). The weight difference between groups is thus in part attributable to the reduced fat mass in hypoxic animals. Under hypoxia, gastrocnemius muscle weight rapidly decreased to $92 \%$ of control within 4 days (d4 vs d2 $\mathrm{P}<0.05$ ) (Figure $2 \mathrm{D}$ ). On day 21 , muscle weight was decreased to $88 \%$ of control (d21 vs d2 $P<0.01$, d21 vs $\mathrm{d} 4 \mathrm{P}<0.05)$. Gastrocnemius weight in the PF-group was unchanged on day 4 , but had declined to $95 \%$ of control on day 21 (d21 vs $d 4 P=0.09$ ). The rapid, initial muscle atrophy following exposure to hypoxia is, therefore, independent of the decreased food intake, whereas the reduction of muscle weight during chronic hypoxia is partially attributable to the lowered food intake. These data indicate that the decrease in body weight and the muscle atrophy in response to hypoxia include a hypoxia-specific component. 


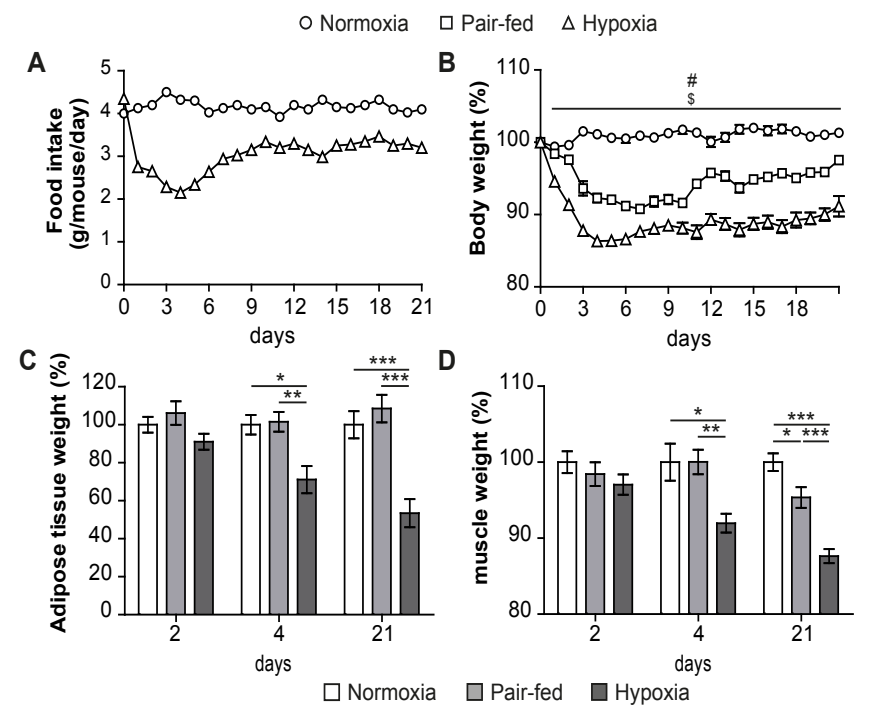

Figure 2. Decreases in body, muscle and adipose weight following hypoxia indicate a hypoxia-specific component. Mice were exposed to normoxia $(\mathrm{N})$ or hypoxia $(\mathrm{H})$ or to normoxia with pair-feeding (PF) to hypoxic mice. Mice were sacrificed at the indicated time points and tissue weights were determined. (A) Food intake per animal per day. (B) Body weight as the percentage of the start weight. Significant differences $(\mathrm{P}<0.05)$ between groups $\mathrm{H}$ and $\mathrm{N}$ are indicated by a dollar sign $\$$ and between groups $\mathrm{H}$ and PF by a hash \#. (C) Epididymal fat pad weight corrected for start bodyweight expressed as the percentage of the normoxic value for the corresponding time point. (D) Gastrocnemius muscle weight corrected for start body weight expressed as the percentage of the normoxic value for the corresponding time point. Significant differences between groups at a given time point are indicated by asterisks, with ${ }^{*}=P \leq 0.05,{ }^{* *}=P \leq 0.01$ or ${ }^{* *}=P \leq 0.001$. Trends are indicated by the specific $P$-value. ( $n=6-8$ per group).

\section{Hypoxia activates UPR, but does not inhibit regulatory effectors of protein synthesis.}

It was shown previously in vitro that hypoxia induces the phosphorylation (inactivation) of EIF2 $\alpha$ [18]. We therefore analyzed the phosphorylation status of EIF2 $\alpha$ in control mice (normoxic or pair-fed) and mice exposed to hypoxia. Contrary to expectations, quantification of phosphorylated (Ser51) and total EIF2a protein revealed a decrease in the degree of phosphorylation in hypoxic versus pair-fed and normoxic animals, suggesting a permissive rather than inhibitory effect of hypoxia on protein synthesis (Figure $3 A, G$ ). Despite the reduced phosphorylation of EIF2 $\alpha$, mRNA expression of its downstream target activating transcription factor 4 (Atf4) was elevated by hypoxia at day 4 and 21 (Figure 3B). Increased ATF4 protein expression induces upregulation of downstream targets such as protein phosphatase 1, regulatory subunit 15A (PPP1R15A, a.k.a. GADD34), which is part of a complex that dephosphorylates EIF2 $\alpha$ [41]. We indeed found increased mRNA expression of Gadd34 at days 4 and 21 (Figure 3C), in agreement with an increase of ATF4 protein concentrations. The acute increase in Gadd34 ex- 
pression could be mimicked by food restriction alone, whereas the later increase was attributable to hypoxia. When tested in vitro, hypoxia also induced a $\sim 2$-fold upregulation of Atf4 and Gadd34 mRNA in differentiated myotubes (Fig. 3D), indicating that Atf4 and Gadd34 can be directly induced by hypoxia in muscle. Thus, despite activation of downstream targets of the UPR in muscle in response to hypoxia, the low phosphorylation levels of EIF2 $\alpha$ suggest a permissive state for mRNA translation.

\section{Hypoxia increases mTOR-related facilitation of protein synthesis.}

Increased protein synthesis requires an increased phosphorylation of the markers 4E-BP1 and P70S6K1 [17]. Food restriction reduced protein synthesis as shown by a transient decrease of 4E-BP1 (Ser65) and P70S6K1 (Thr389) phosphorylation (Figure 3E, F and G). Remarkably, phosphorylation levels of these proximal regulators of mRNA translation were maintained during the acute response to hypoxia (day 4) and even increased (4E-BP1) at day 21 (late response). Total protein content of 4E-BP1 remained unchanged (Figure 3E, G), whereas that of P70S6K1 had decreased on day 21 (Figure 3F, G). These data show that 4E-BP1 and $\mathrm{P} 70 \mathrm{~S} 6 \mathrm{~K} 1$ phosphorylation was maintained or even increased in response to hypoxia despite the suppressive effects of decreased food intake.

\section{Hypoxia affects AKT signaling during the acute and chronic response.}

AKT plays a major role in the regulation of muscle metabolism, as it can both stimulate protein synthesis via mTOR and inhibition of GSK3 $\beta$, and prevent protein degradation through the inhibitory phosphorylation of forkhead box protein O1 (FOXO1) [34, 42, 43]. Short-term hypoxia decreased the absolute amount of phosphorylated AKT (Ser473), and increased the concentration of total AKT, so that the degree of phosphorylation of AKT (ratio p-AKT/total AKT) decreased (Figure 4A, F). Long-term hypoxia further increased the abundance of total AKT, without further affecting the degree of phosphorylation. In contrast, p-AKT was strongly increased in muscle of pair-fed mice, suggesting that hypoxia counteracted the activation of AKT that was induced by food restriction. Although not significantly different in all instances from control conditions, the phosphorylation status of the downstream substrates of AKT, that is GSK3 $\beta$ (Figure 4B, F) and FOXO1 (Figure 4C, F), corresponded with the alterations in AKT phosphorylation, and therefore likely reflected AKT activity. In addition, total FOXO1 protein levels were increased in response to chronic hypoxia. The increased FOXO1 protein concentration corresponded with the rapid increase in Foxo1 mRNA expression during hypoxia (Figure 4D). Hypoxia also increased Foxo1 mRNA expression in cultured muscle cells, indicating a cell-autonomous response to hypoxia (Figure 4E). 

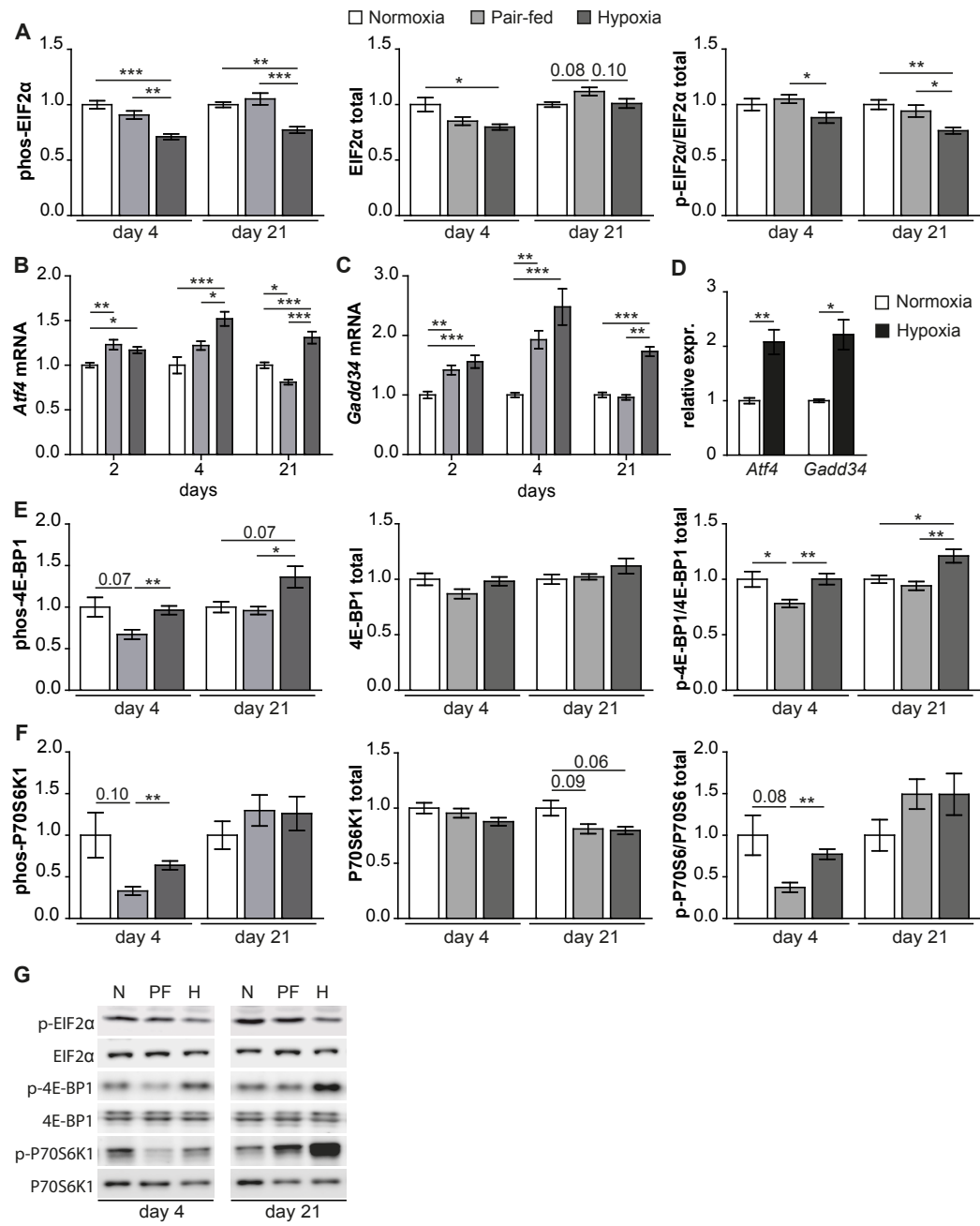

Figure 3. Hypoxia does not inhibit protein synthesis through UPR or mTORC1 signaling. Phosphorylation of proteins and expression of genes in skeletal muscle involved in UPR signaling: (A) phosphorylated EIF2 $\alpha$ (left panel), total EIF2 $\alpha$ (middle panel), ratio phosphorylated EIF2 $\alpha$ (Ser51) over total EIF2 $\alpha$ (right panel). (B) Atf4 and (C) Gadd34 mRNA expression in vivo. (D) Atf4 and Gadd34 mRNA expression in hypoxia-exposed myotubes $(n=3)$. Proteins regulated by mTORC1 in skeletal muscle: (E) Phosphorylated (Ser65) 4E-BP1 (left panel), total 4E-BP1 (middle panel), ratio of phosphorylated (Ser65) 4E-BP1 over total 4E-BP1 (right panel). (F) Phosphorylated (Thr389) P70S6K1 (left panel), total P70S6K1 (middle panel), ratio of phosphorylated (Thr389) P70S6K1 over total P70S6K1 (right panel). (G) Representative Western blots of the indicated proteins. mRNA concentration was corrected for 18S RNA concentration and normalized to the normoxic value for the corresponding time point. Protein concentrations were corrected for total protein loaded and normalized to normoxic values for the corresponding time point. Significant differences between groups at a given time point are indicated by asterisks, with ${ }^{*}=P \leq 0.05,{ }^{* *}=P \leq 0.01$ or ${ }^{* * *}=P \leq 0.001$. Trends are indicated by the specific $P$-value ( $n=6-8$ per group). 
A
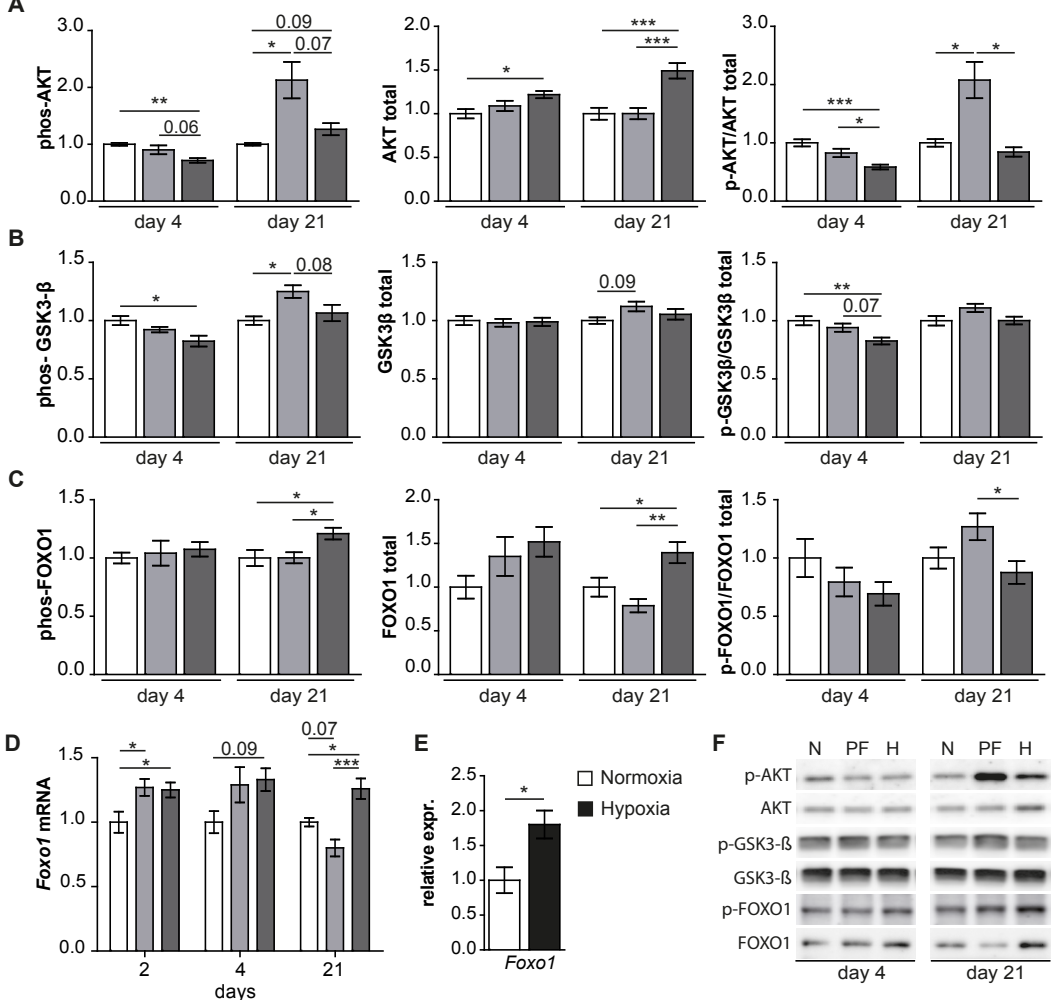

Figure 4. Hypoxia affects AKT signaling during the acute and chronic response. (A) Phosphorylated AKT (Ser473) (left panel), total AKT (middle panel), ratio of phosphorylated AKT (Ser473) over total AKT (right panel). (B) Phosphorylated GSK3 $\beta$ (Ser9) (left panel), total GSK3 $\beta$ (middle panel), ratio of phosphorylated GSK3 $\beta$ (Ser9) over total GSK3 $\beta$. (C) phosphorylated FOXO1 (Ser256) (left panel), total FOXO1 (middle panel), ratio of phosphorylated FOXO1 (Ser256) over total FOXO1. (D) Foxo1 mRNA expression in gastrocnemius muscle. (E) Foxo1 mRNA expression in hypoxia-exposed myotubes $(n=3)$. ( $F)$ Representative Western blots of the indicated proteins. mRNA concentrations were corrected for $18 \mathrm{~S}$ mRNA concentration and normalized to normoxic values for the corresponding time points. Protein concentration was corrected for total protein loaded and normalized to normoxic values for the corresponding time points. Significant differences between groups at a given time point are indicated by asterisks with ${ }^{*}=P \leq 0.05,{ }^{* *}=P \leq 0.01$ or ${ }^{* * *}=P \leq 0.001$. Trends are indicated by the specific $P$-value ( $n=6-8$ per group). 


\section{Hypoxia activates proteasomal and lysosomal protein degradation.}

Gene expression levels of markers of proteasomal and lysosomal protein degradation were determined. Hypoxia caused a rapid and transient increase in the expression of the proteasomal degradation gene Murf1 mRNA, which was completely mimicked by food restriction (Figure 5A), whereas the increased expression of Atrogin-1 mRNA was only in part dependent on reduced food intake on days 2 and 4 of the hypoxia treatment (Figure 5B). Only Atrogin- 1 was upregulated in response to chronic hypoxia. Expression of Bnip3 and Map1/c3b mRNAs, which are markers of lysosomal protein degradation, was increased in response to hypoxia (Figure $5 \mathrm{C}$ and D). Semi-starvation only accounted for the initial, but not the chronic increase of Map1/c3B and Bnip3 expression. A cell-autonomous effect of hypoxia was only apparent on Bnip3 mRNA expression in C2C12 myotubes (Figure 5E), suggesting that Murf1, Atrogin-1 and Map1/c3B expression in hypoxia-exposed skeletal muscle are regulated through an indirect mechanism. Together, these data showed an increased expression of genes of the proteasomal and the lysosomal protein degradation pathways, suggesting increased protein degradation during the acute and chronic response to hypoxia.

\section{Discussion}

To address the potential contribution of hypoxia to the development of muscle wasting associated with COPD, signaling pathways involved in protein turnover were systematically studied in a mouse model of normobaric hypoxia and in differentiated myotubes of a murine skeletal myoblast line. Our data indicate an altered regulation of protein turnover during hypoxia-induced skeletal muscle atrophy, which was in part due to hypoxia-induced semi-starvation, but also involved a hypoxia-specific component.

\section{Rationale for the mouse model.}

It has been demonstrated previously that hypobaric hypoxia results in muscle atrophy [44, 45]. Although elevated protein turnover has been shown in rats under hypobaric hypoxia [46], changes in signaling pathways of protein synthesis and degradation were not investigated. Since hypobaric hypoxia involves effects of both oxygen limitation and barometric pressure reduction, the latter may confound the interpretation of the effects of hypoxia per se. It was indeed shown that hypobaric hypoxia resulted in greater hypoxemia, hypocapnia, and blood alkalosis than normobaric hypoxia and that these changes were related to the reduction in barometric pressure [47]. Our mouse model, therefore, better mimics the acute and chronic normobaric hypoxic conditions seen in COPD patients and patients with chronic respiratory failure due to other diseases. 


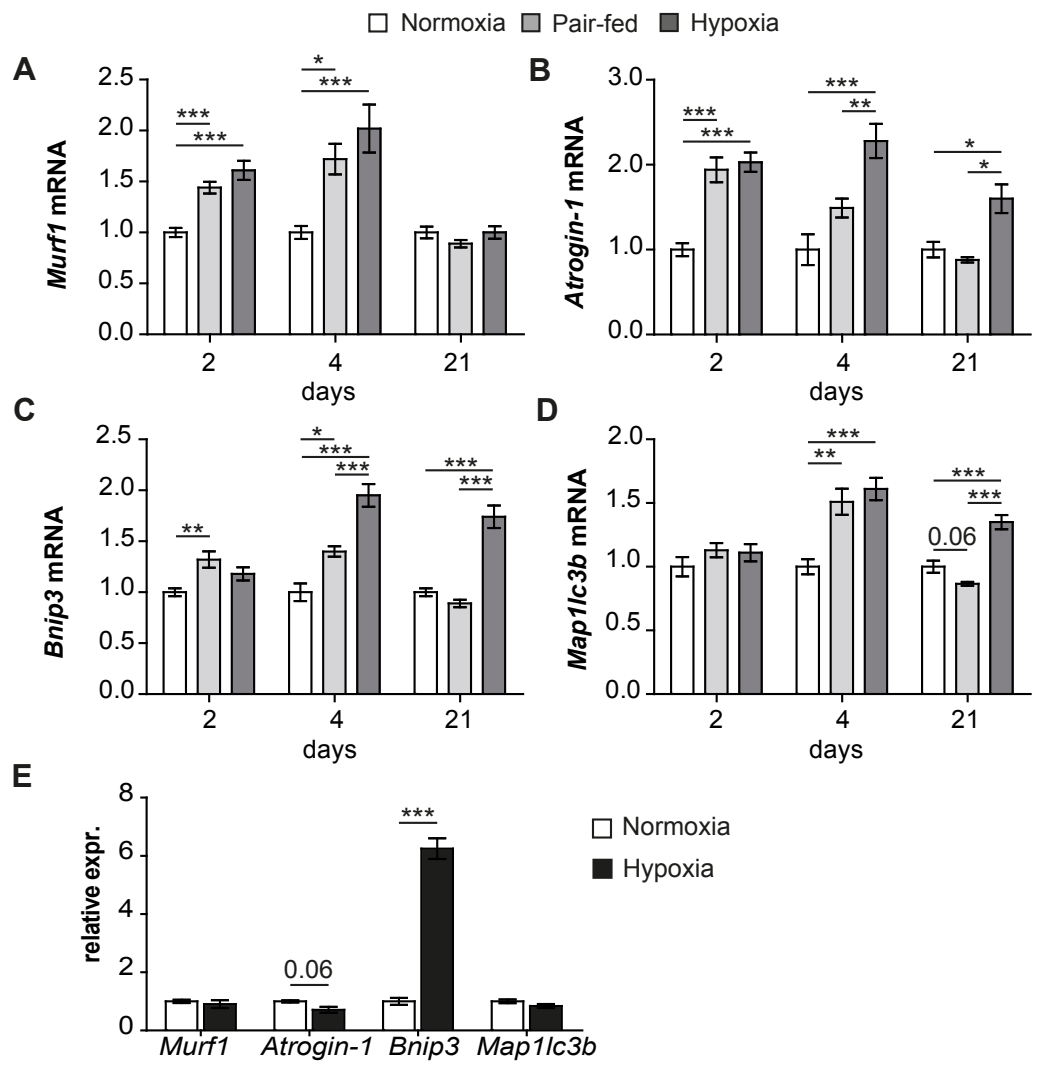

Figure 5. Hypoxia activates proteasomal and lysosomal protein degradation. mRNA expression levels of genes involved in proteasomal protein degradation were measured. (A) Murf1 mRNA expression. (B) Atrogin-1 mRNA expression. Expression of mRNAs of proteins involved in autophagy signaling: (C) Bnip3. (D) Map1/c3b. (E) mRNA expression in myotubes exposed to an ambient oxygen concentration of $4 \%(n=3)$. mRNA concentrations were corrected for 18S RNA concentration and normalized to normoxic values for the corresponding time points. Significant differences between groups at a given time point are indicated by asterisks, with ${ }^{*}=P \leq 0.05,{ }^{* *}=P \leq 0.01$ or ${ }^{* * *}=P \leq 0.001$. Trends are indicated by the specific P-value.

\section{Validation of the mouse model.}

The stepwise decrease of ambient oxygen concentrations resulted in the expected rapid reduction of blood $\mathrm{PaO} 2$. The subsequent decrease in $\mathrm{SaO} 2$ corresponded with the decline in $\mathrm{pH}$ [48]. Prolonged hypoxia resulted in an even lower $\mathrm{SaO} 2$, possibly due to hypoxia-related adaptations such as pulmonary hypertension [49], a common feature of chronic respiratory failure [50]. The continuous increase in erythrocytes reflected the physiological response to counterbalance hypoxemia by increasing the capacity for oxygen transport [51]. Although the $\mathrm{PaO} 2$ of hypoxic mice in this study was lower than that observed in hypoxemic patients, we chose these stringent hypoxic conditions in order to induce more 
profound effects of tissue hypoxia on muscle mass regulation (in a relatively short experimental period). We did not measure tissue oxygenation directly, but the increases observed in the expression of the hypoxia-sensitive genes $\mathrm{Ca}-9$ and Glut1 indicate that hypoxic conditions developed in the gastrocnemius muscle. Interestingly, these two genes showed a different temporal regulation, with Glut1 expression increasing rapidly and transiently and $\mathrm{Ca}-9$ expression increasing only during chronic hypoxia exposure as measured on day 21 . The differential regulation of these two HIF1- $\alpha$ target genes suggested a biphasic adaptive response to hypoxia, hereafter referred to as acute and chronic phases. Both genes were also upregulated in a culture of differentiated hypoxic myotubes, confirming a cell autonomous effect of hypoxia on muscle cells. This mouse model is therefore suitable to investigate the acute and chronic adaptations of skeletal muscle to tissue hypoxia that may occur in patients during acute or chronic respiratory failure.

\section{Hypoxia-induced reduction of food intake.}

Malnutrition is a prevalent problem in chronic respiratory diseases. Since a negative energy balance affects muscle mass, we measured food intake in our study. The present findings show that, in agreement with literature [13], hypoxemia is an important causal factor in reduced energy uptake, as hypoxia induced a dramatic, but transient reduction in food intake in our model. Even though food intake slowly recovered, it remained lower than in the normoxic group throughout the experiment, stabilizing at around $80 \%$ of the control intake. A similar decrease in food intake has been described earlier with milder normobaric hypoxia (11\% O2) [52]. Decreased appetite was also reported in rats and healthy volunteers exposed to hypobaric hypoxia and also led to reductions in body weight and muscle mass $[14,15]$. Similar to the expression of HIF1- $\alpha$ marker genes (Glut1 and Ca-9) [38, 53], the food intake pattern suggested an acute (up to four days) and a chronic phase in the adaptation to hypoxia.

\section{Acute effects of hypoxia.}

Subsequent comparisons to a normoxic group of mice, pair-fed to the hypoxic animals, allowed us to separate the contributions of reduced food intake from the specific effects of hypoxia on muscle atrophy. Food restriction during the acute phase alone resulted in an acute inhibition of regulatory steps of protein synthesis in muscle, such as decreased phosphorylation of 4E-BP1 and P70S6K1. This observation is in line with previous reports showing suppressed mTORC1 signaling and protein synthesis in response to food restriction [54, 55]. Although inhibition of mTOR activity has been demonstrated during hypoxia in vitro [56], we did not find inhibition of mTOR signaling in vivo, as phosphorylation of 4E-BP1 and P70S6K1 was maintained in hypoxic mice in contrast to pair-fed normoxic 
mice. Apparently, hypoxia suppresses the inhibitory effects of semi-starvation on mTOR signaling. This relief of protein synthesis was also reflected in the hypoxiainduced decrease of EIF2 $\alpha$ phosphorylation and suggested that cap-dependent translation remains active under hypoxia even though similar or even increased expression of downstream targets of the stress pathway were found. In addition, an increased expression of genes involved in proteasomal or lysosomal protein degradation (Murf1, Atrogin-1, Map1/c3b and Bnip3) by hypoxia was observed, suggesting an enhanced muscle protein breakdown as described previously [31, 57-59]. Whereas expression of Murf1and Map1/c3b mRNA was entirely dependent on food restriction during the acute response, expression of Bnip3 and Atrogin-1 mRNAs was further increased by hypoxia suggesting a hypoxia-specific effect. Bnip3 expression was also increased in hypoxia-exposed myotubes suggesting direct regulation by oxygen deficiency. Indeed, the Bnip3 promoter contains a functional hypoxia response element (HRE) [30], supporting the notion that HIF1a stabilisation occurred during hypoxia in skeletal muscle in vivo. Despite induction of increased proteolytic capacity, as shown by Murf1, Atrogin-1, Map1/c3b and Bnip3 expression and suppressed protein synthesis signaling, semi-starvation did not result in muscle weight loss during the acute phase. Acute hypoxia, on the other hand, induced an even greater loss of body weight than mere food restriction alone and this was also reflected in muscle- and fatpad weight loss. In summary, hypoxia had a strong catabolic effect during the acute phase of hypoxia, whereas food restriction did not affect muscle weight. Despite differences on muscle mass, acute hypoxia and food restriction both increased regulatory cues of proteasomal and lysosomal protein degradation. In addition, hypoxia relieved suppression of protein synthesis signaling by reduced food intake.

\section{Chronic effects of hypoxia.}

As expected, the partial recovery of food intake led to a normalization of regulatory steps in protein synthesis and protein degradation. Accordingly, pair-fed animals did not differ from their normoxic controls with respect to parameters of mTORC1 signaling, lysosomal or proteasomal protein degradation signaling or stress kinase signaling. Pair-fed animals did show increased phosphorylation of AKT and downstream targets, which we interpret as a relief of mTOR signaling, and a suppression of the induction of genes involved in protein degradation. Together, this could result in a partial recovery of muscle mass relative to the initial muscle mass loss. Despite the slow regain in body weight and the altered regulation of protein turnover, pair-fed animals did show a residual decrease in muscle weight at the end of the chronic phase. Interestingly, fat tissue weight remained constant. Hypoxic animals, on the other hand, continued to lose both muscle and 
adipose tissue despite similar rates of body weight gain. Accordingly, they did not recover from the muscle loss during the acute phase of hypoxia. The sustained activation status of protein synthesis and degradation and the continuous loss of tissue weight, when compared to control and pair-fed animals, may reflect an elevated protein turnover and subsequent higher energy consumption under sustained hypoxic conditions. Although future studies should address actual protein synthesis and degradation rates and compare them to the observed changes in protein turnover signaling, elevated protein turnover rates in response to hypoxia described by Chaudhary et al. [46] correspond with the increased expression of genes involved in protein turnover that we report here.

\section{Conclusion}

Hypoxia-induced atrophy involves food intake- and hypoxia-dependent effects (Figure 6). We could also distinguish acute and chronic adaptive responses to hypoxia. Acute, severe hypoxia but not food restriction alone led to a rapid loss of muscle and fat tissue mass. During chronic hypoxia a mild, continuous loss of muscle and fat mass was observed. Overall, hypoxia counteracted the inhibitory effects of reduced food intake on protein synthesis signaling, and induced the expression of genes involved in proteasomal and lysosomal protein degradation. As expected, normobaric hypoxia-induced muscle atrophy was indeed partially a result of reduced food intake. Furthermore, contrary to our hypothesis, normobaric hypoxia-induced muscle atrophy involves activation of protein synthesis pathways in addition to increased expression of genes involved in protein degradation.
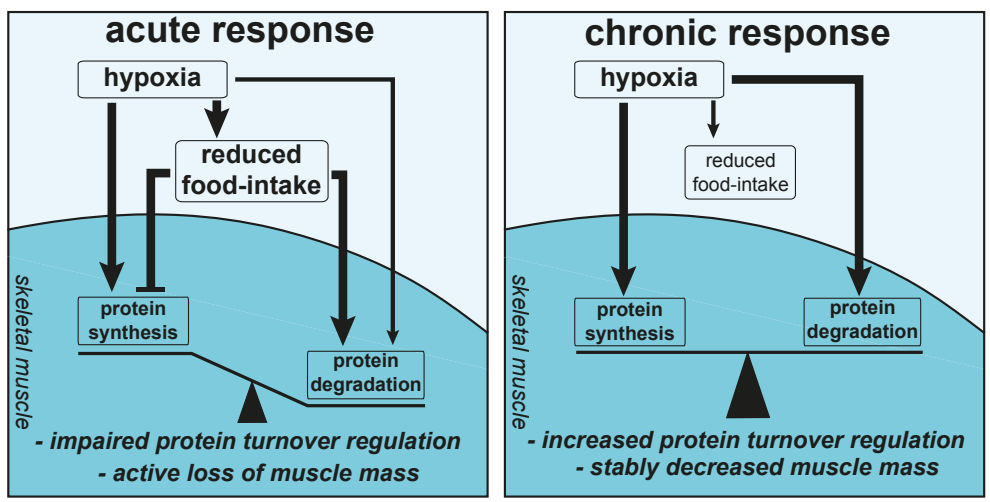

Figure 6. Schematic representation of the effects of hypoxia and reduced food intake on the regulation of skeletal muscle protein turnover. 


\section{References}

1. Soguel Schenkel N, Burdet L, de Muralt B, Fitting JW. Oxygen saturation during daily activities in chronic obstructive pulmonary disease. The European respiratory journal. 1996;9(12):25849.

2. Wouters EF. Nutrition and metabolism in COPD. Chest. 2000;117(5 Suppl 1):274S-80S.

3. Mador MJ, Bozkanat E. Skeletal muscle dysfunction in chronic obstructive pulmonary disease. Respir Res. 2001;2(4):216-24.

4. Jagoe RT, Engelen MP. Muscle wasting and changes in muscle protein metabolism in chronic obstructive pulmonary disease. Eur Respir J Suppl. 2003;46:52s-63s.

5. Decramer M, De Benedetto F, Del Ponte A, Marinari S. Systemic effects of COPD. Respiratory medicine. 2005;99 Suppl B:S3-10.

6. Gray-Donald K, Gibbons L, Shapiro SH, Macklem PT, Martin JG. Nutritional status and mortality in chronic obstructive pulmonary disease. American journal of respiratory and critical care medicine. 1996;153(3):961-6.

7. Boyer SJ, Blume FD. Weight loss and changes in body composition at high altitude. Journal of applied physiology: respiratory, environmental and exercise physiology. 1984;57:1580-5.

8. Hoppeler H, Kleinert E, Schlegel C, Claassen H, Howald H, Kayar SR, et al. Morphological adaptations of human skeletal muscle to chronic hypoxia. Int J Sports Med. 1990;11 Suppl 1:S3-9.

9. Favier FB, Costes F, Defour A, Bonnefoy R, Lefai E, Baugé S, et al. Down-regulation of Akt/ mammalian target of rapamycin pathway in skeletal muscle is associated with increased REDD1 expression in response to chronic hypoxia. American journal of physiology Regulatory, integrative and comparative physiology. 2010:1659-66.

10. Hayot M, Rodriguez J, Vernus B, Carnac G, Jean E, Allen D, et al. Myostatin up-regulation is associated with the skeletal muscle response to hypoxic stimuli. Molecular and cellular endocrinology. 2010.

11. Millet GP, Faiss R, Pialoux V. Point: Hypobaric hypoxia induces different physiological responses from normobaric hypoxia. J Appl Physiol. 2012;112(10):1783-4.

12. Mounier R, Brugniaux JV. Counterpoint: Hypobaric hypoxia does not induce different responses from normobaric hypoxia. J Appl Physiol. 2012;112(10):1784-6.

13. Raguso $\mathrm{Ca}$, Luthy $\mathrm{C}$. Nutritional status in chronic obstructive pulmonary disease: role of hypoxia. Nutrition (Burbank, Los Angeles County, Calif). 2011;27:138-43.

14. Westerterp-plantenga MS, Westerterp KR, Rubbens M, Verwegen RT, Richelet J-p, Gardette B, et al. Appetite at " high altitude " [ Operation Everest III ( Comex- ' 97 )]: a simulated ascent of Mount Everest effects of caloric restriction J Appl Physiol (1985). 2011:391-9.

15. Chen XQ, Dong J, Niu CY, Fan JM, Du JZ. Effects of hypoxia on glucose, insulin, glucagon, and modulation by corticotropin-releasing factor receptor type 1 in the rat. Endocrinology. 2007;148(7):3271-8.

16. Macdonald JH, Oliver SJ, Hillyer K, Sanders S, Smith Z, Williams C, et al. Body composition at high altitude: a randomized placebo-controlled trial of dietary carbohydrate supplementation. The American journal of clinical nutrition. 2009;90(5):1193-202. 
17. Ma XM, Blenis J. Molecular mechanisms of mTOR-mediated translational control. Nat Rev Mol Cell Biol. 2009;10:307-18.

18. Ron D. Translational control in the endoplasmic reticulum stress response. The Journal of clinical investigation. 2002;110(10):1383-8.

19. Gingras AC, Raught B, Gygi SP, Niedzwiecka A, Miron M, Burley SK, et al. Hierarchical phosphorylation of the translation inhibitor 4E-BP1. Genes \& development. 2001;15(21):2852-64.

20. Gingras aC, Gygi SP, Raught B, Polakiewicz RD, Abraham RT, Hoekstra MF, et al. Regulation of 4E-BP1 phosphorylation: a novel two-step mechanism. Genes \& development. 1999;13:142237.

21. Holz MK, Ballif BA, Gygi SP, Blenis J. mTOR and S6K1 mediate assembly of the translation preinitiation complex through dynamic protein interchange and ordered phosphorylation events. Cell. 2005;123(4):569-80.

22. Koritzinsky M, Rouschop KMa, van den Beucken T, Magagnin MG, Savelkouls K, Lambin P, et al. Phosphorylation of elF2alpha is required for mRNA translation inhibition and survival during moderate hypoxia. Radiotherapy and oncology : journal of the European Society for Therapeutic Radiology and Oncology. 2007;83:353-61.

23. Koumenis C, Naczki C, Koritzinsky M, Rastani S, Diehl A, Sonenberg N, et al. Regulation of Protein Synthesis by Hypoxia via Activation of the Endoplasmic Reticulum Kinase PERK and Phosphorylation of the Translation Initiation Factor elF2 $\alpha$. Society. 2002;22:7405-16.

24. Glass DJ. Skeletal muscle hypertrophy and atrophy signaling pathways. The international journal of biochemistry \& cell biology. 2005;37(10):1974-84.

25. Gomes MD, Lecker SH, Jagoe RT, Navon A, Goldberg AL. Atrogin-1, a muscle-specific F-box protein highly expressed during muscle atrophy. Proc Natl Acad Sci U S A. 2001;98(25):144405.

26. Jagoe RT, Goldberg AL. What do we really know about the ubiquitin-proteasome pathway in muscle atrophy? Curr Opin Clin Nutr Metab Care. 2001;4(3):183-90.

27. Lecker SH, Solomon V, Mitch WE, Goldberg AL. Muscle protein breakdown and the critical role of the ubiquitin-proteasome pathway in normal and disease states. J Nutr. 1999;129(1S Suppl):227S-37S.

28. McLoughlin TJ, Smith SM, DeLong AD, Wang H, Unterman TG, Esser KA. FoxO1 induces apoptosis in skeletal myotubes in a DNA-binding-dependent manner. American journal of physiology Cell physiology. 2009;297(3):C548-55.

29. Sandri M. Autophagy in skeletal muscle. FEBS letters. 2010;584(7):1411-6.

30. Bellot G, Garcia-Medina R, Gounon P, Chiche J, Roux D, Pouysségur J, et al. Hypoxia-induced autophagy is mediated through hypoxia-inducible factor induction of BNIP3 and BNIP3L via their BH3 domains. Mol Cell Biol. 2009;29:2570-81.

31. Rouschop KMA, Beucken TVD, Dubois L, Niessen H, Bussink J, Savelkouls K, et al. The unfolded protein response protects human tumor cells during hypoxia through regulation of the autophagy genes MAP1LC3B and ATG5. Cancer. 2010;120:127-41. 
32. Wang X, Ma S, Qi G. Effect of hypoxia-inducible factor 1-alpha on hypoxia/reoxygenationinduced apoptosis in primary neonatal rat cardiomyocytes. Biochemical and biophysical research communications. 2012;417(4):1227-34.

33. Du K, Tsichlis PN. Regulation of the Akt kinase by interacting proteins. Oncogene. 2005;24(50):7401-9.

34. Stitt TN, Drujan D, Clarke Ba, Panaro F, Timofeyva Y, Kline WO, et al. The IGF-1/PI3K/Akt Pathway Prevents Expression of Muscle Atrophy-Induced Ubiquitin Ligases by Inhibiting FOXO Transcription Factors. Mol Cell. 2004;14:395-403.

35. Liu Y, Cox SR, Morita T, Kourembanas S. Hypoxia regulates vascular endothelial growth factor gene expression in endothelial cells. Identification of a $5^{\prime}$ enhancer. Circulation research. 1995;77(3):638-43.

36. McClung JM, Judge AR, Talbert EE, Powers SK. Calpain-1 is required for hydrogen peroxideinduced myotube atrophy. American journal of physiology Cell physiology. 2009;296(2):C36371.

37. Wolfe HG. Blood-pH differences in two inbred strains of mice. J Hered 1959. ;50:155-8.

38. Wykoff CC, Beasley NJ, Watson PH, Turner KJ, Pastorek J, Sibtain A, et al. Hypoxia-inducible expression of tumor-associated carbonic anhydrases. Cancer Res. 2000;60(24):7075-83.

39. Airley R, Loncaster J, Davidson S, Bromley M, Roberts S, Patterson A, et al. Glucose transporter glut-1 expression correlates with tumor hypoxia and predicts metastasis-free survival in advanced carcinoma of the cervix. Clin Cancer Res. 2001;7(4):928-34.

40. Hoppeler H, Vogt M, Weibel ER, Fluck M. Response of skeletal muscle mitochondria to hypoxia. Experimental physiology. 2003;88(1):109-19.

41. Ma Y, Hendershot LM. Delineation of a negative feedback regulatory loop that controls protein translation during endoplasmic reticulum stress. J Biol Chem. 2003;278(37):34864-73.

42. Bodine SC, Stitt TN, Gonzalez M, Kline WO, Stover GL, Bauerlein R, et al. Akt/mTOR pathway is a crucial regulator of skeletal muscle hypertrophy and can prevent muscle atrophy in vivo. Nat Cell Biol. 2001;3:1014-9.

43. Sandri M, Sandri C, Gilbert A, Skurk C, Calabria E, Picard A, et al. Foxo transcription factors induce the atrophy-related ubiquitin ligase atrogin-1 and cause skeletal muscle atrophy. Cell. 2004;117:399-412.

44. Viganò A, Ripamonti M, De Palma S, Capitanio D, Vasso M, Wait R, et al. Proteins modulation in human skeletal muscle in the early phase of adaptation to hypobaric hypoxia. Proteomics. 2008;8:4668-79.

45. Magalhães J, Ascensão A, Soares JMC, Ferreira R, Neuparth MJ, Marques F, et al. Acute and severe hypobaric hypoxia increases oxidative stress and impairs mitochondrial function in mouse skeletal muscle. Journal of applied physiology (Bethesda, Md : 1985). 2005;99:124753.

46. Chaudhary P, Suryakumar G, Prasad R, Singh SN, Ali S, llavazhagan G. Chronic hypobaric hypoxia mediated skeletal muscle atrophy: role of ubiquitin-proteasome pathway and calpains. Mol Cell Biochem. 2012. 
47. Savourey G, Launay J-C, Besnard Y, Guinet A, Travers S. Normo- and hypobaric hypoxia: are there any physiological differences? European journal of applied physiology. 2003;89:122-6.

48. Gray LH, Steadman JM. Determination of the oxyhaemoglobin dissociation curves for mouse and rat blood. Journal of Physiology. 1964;175:161-71.

49. Steudel W, Scherrer-Crosbie M, Bloch KD, Weimann J, Huang PL, Jones RC, et al. Sustained pulmonary hypertension and right ventricular hypertrophy after chronic hypoxia in mice with congenital deficiency of nitric oxide synthase 3 . The Journal of clinical investigation. 1998;101(11):2468-77.

50. Naeije R, Barbera JA. Pulmonary hypertension associated with COPD. Crit Care. 2001;5(6):2869.

51. Haase VH. Hypoxic regulation of erythropoiesis and iron metabolism. Am J Physiol Renal Physiol. 2010;299(1):F1-13.

52. Kozak W, Wrotek S. Hypoxia-induced sickness beha viour. Pharmacology. 2006:35-50.

53. Ebert BL, Firth JD, Ratcliffe PJ. Hypoxia and mitochondrial inhibitors regulate expression of glucose transporter-1 via distinct Cis-acting sequences. J Biol Chem. 1995;270(49):29083-9.

54. Ogata T, Oishi Y, Higuchi M, Muraoka I. Fasting-related autophagic response in slow- and fasttwitch skeletal muscle. Biochemical and biophysical research communications. 2010;394:13640.

55. Haj AJEL, Lewis SEM, Goldspink DF, Merry BJ, Holehan AM. The effect of chronic and acute dietary restriction on the growth and protein turnover of fast and slow types of rat skeletal muscle. 1986;85:281-7.

56. Arsham AM, Howell JJ, Simon MC. A novel hypoxia-inducible factor-independent hypoxic response regulating mammalian target of rapamycin and its targets. J Biol Chem. 2003;278:2965560.

57. Razeghi P, Baskin KK, Sharma S, Young ME, Stepkowski S, Essop MF, et al. Atrophy, hypertrophy, and hypoxemia induce transcriptional regulators of the ubiquitin proteasome system in the rat heart. Biochemical and biophysical research communications. 2006;342:361-4.

58. Zhang H, Bosch-Marce M, Shimoda LA, Tan YS, Baek JH, Wesley JB, et al. Mitochondrial autophagy is an HIF-1-dependent adaptive metabolic response to hypoxia. $\mathrm{J}$ Biol Chem. 2008;283(16):10892-903.

59. Gamboa JL, Andrade FH. Mitochondrial content and distribution changes specific to mouse diaphragm after chronic normobaric hypoxia. American journal of physiology Regulatory, integrative and comparative physiology. 2010;298:R575-83. 


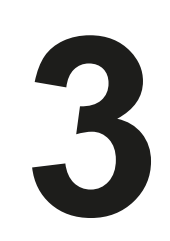

\section{Differential sensitivity of oxidative and glycolytic muscles to hypoxia-induced muscle atrophy}

C.C. de Theije, R.C.J. Langen, W.H. Lamers, H.R. Gosker, A.M.W.J. Schols, S.E. Köhler

J Appl Physiol (1985). 2015 Jan 15;118(2):200-211 


\section{Abstract}

Hypoxia as a consequence of acute and chronic respiratory disease has been associated with muscle atrophy. This study investigated the sensitivity of oxidative and glycolytic muscles to hypoxia-induced muscle atrophy. Male mice were exposed to $8 \%$ normobaric oxygen for up to 21 days. Oxidative soleus and glycolytic extensor digitorum longus (EDL) muscles were isolated, weighed and assayed for gene expression profiles of the ubiquitin-proteasome system (UPS), the autophagy-lysosome pathway (ALP), and glucocorticoid receptor (GR)- and hypoxia-inducible factor-1 $\alpha$ (HIF1 $\alpha$ ) signaling. Fiber-type composition and the capillary network were investigated. Hypoxia-induced muscle atrophy was more prominent in the EDL than the soleus muscle. Although increased expression of HIF1 $\alpha$ target genes showed that both muscle types sensed hypoxia, their adaptive responses differed. Atrophy consistently involved a hypoxia-specific effect (i.e. not attributable to a hypoxia-mediated reduction of food intake) in the EDL only. Hypoxia-specific activation of the UPS and ALP and increased expression of the glucocorticoid receptor $(G r)$ and its target genes were also mainly observed in the EDL. In the soleus, stimulation of gene expression of those pathways could be mimicked to a large extent by food restriction alone. Hypoxia increased the number of capillary contacts per fiber cross-sectional area in both muscles. In the EDL, this was due to type II fiber atrophy, whereas in the soleus the absolute number of capillary contacts increased. These responses represent two distinct modes to improve oxygen supply to muscle fibers, but may aggravate muscle atrophy in COPD patients who have a predominance of type II fibers. 


\section{Introduction}

Weight loss and muscle atrophy are common features associated with respiratory diseases such as advanced chronic obstructive pulmonary disease (COPD) [1-3], acute exacerbations of COPD [4], idiopathic pulmonary arterial hypertension [5], and acute respiratory distress syndrome (ARDS) [6]. Loss of muscle mass is a strong predictor of mortality and significantly increases disease burden [1, 2, 7]. Hypoxia is well-known for its negative effect on muscle mass [8]. Episodes of both acute and chronic hypoxia are hallmarks of the above mentioned respiratory disorders [9], therefore, understanding the mechanisms by which hypoxia induces muscle atrophy may benefit disease management. Interestingly, muscle atrophy in advanced COPD patients is characterized by a reduction of the muscle fiber cross-sectional area (FCSA) and particularly affects the glycolytic type IIX and IIA/IIX hybrid fibers [10,11]. Moreover, a fiber-type shift with a decrease of fiber type I (slow, oxidative) and an increase of type II fibers (fast, glycolytic) [11-14] as well as a reduced capillarity [15] are observed, potentially rendering these patients even more vulnerable to hypoxia-induced muscle atrophy. Differential sensitivity of glycolytic and oxidative muscle to atrophy stimuli other than hypoxia has previously been observed in animal models [16-18]. In order to disentangle which muscle (fiber) types are most sensitive to hypoxia-induced muscle atrophy, we analyzed the effects of severe acute and chronic hypoxia on muscle mass and capillarity on the soleus, as a typical example of an oxidative muscle, and the extensor digitorum longus (EDL), as representative for a glycolytic muscle in mice [19]. As oxidative fibers show higher oxygen consumption, we hypothesized that oxidative muscle fibers are less resistant to hypoxia-induced muscle atrophy than glycolytic muscle fibers. To test our hypothesis, mice were exposed to severe hypoxia for either 4 (acute) or up to 21 days (chronic) and compared to control mice kept under normoxic conditions. Since we have previously demonstrated a hypoxia-induced reduction of food intake [20], we also included a pair-fed control group in our experimental design. Muscle atrophy and gene expression profiles of relevant catabolic pathways were determined (Table 1). In addition, muscle capillarity was assessed. Our study shows that both oxidative and glycolytic muscles show structural adaptations to hypoxia that result in a reduced FCSA per capillary contact, and that hypoxia-induced atrophy mainly affects glycolytic fibers. 


\begin{tabular}{|c|c|c|}
\hline Gene & Function & References \\
\hline \multicolumn{3}{|c|}{ Ubiquitin 26S-proteasome system (UPS) } \\
\hline Murf1 (Trim63) & E3 ubiquitin protein ligase & [14] \\
\hline Atrogin1 (Fbxo32) & E3 ubiquitin protein ligase & {$[14,15]$} \\
\hline Nedd4 & E3 ubiquitin protein ligase & [27] \\
\hline \multicolumn{3}{|c|}{ Autophagy-lysosome pathway (ALP) } \\
\hline Bnip3 & Regulation of autophagy/mitophagy & {$[3]$} \\
\hline Map1lc3B & Regulation of autophagy/mitophagy & {$[47]$} \\
\hline $\operatorname{Atg} 5$ & E3 ubiquitin protein ligase & [38] \\
\hline \multicolumn{3}{|c|}{ Glucocorticoid receptor (GR) signaling } \\
\hline Glul & Ammonia and glutamine metabolism & [36] \\
\hline Mstn & Inhibitor of myogenesis & [33] \\
\hline Klf15 & $\begin{array}{l}\text { Transcription factor, upregulates Murf1 } \\
\text { atrogin1 }\end{array}$ & {$[50]$} \\
\hline
\end{tabular}

Table 1. Pathways and marker genes tested in this study.

\section{Materials and Methods}

\section{Animals and tissue collection.}

12-week-old C57BL/6J male mice (Charles River Laboratories International, Wilmington, MA, USA) ( $n=48)$ were randomly divided into 3 groups: normoxia $(\mathrm{N})$, normoxic animals pair-fed to hypoxic animals (PF) and normobaric hypoxia (H). Oxygen levels in the $\mathrm{H}$-group were reduced in a stepwise manner from ambient levels to $12 \%$ on day 1 , to $10 \%$ on day 2 , and finally to $8 \%$ on day 3 , and maintained at $8 \%$ for the remainder of the experiment. Of each group, 8 mice were sacrificed on day 4 ("acute" hypoxia) and another 8 on day 21 ("chronic" hypoxia). The soleus and the EDL muscles were isolated and frozen for immunohistochemical analysis and RNA extraction. Tissue weights were corrected for starting body weight. Procedures are described in more detail in [20]. Briefly, at the start of the experiments, 4 mice were placed in each cage. Mice and food were weighed daily. The food intake/day is an average from 4 mice. The pairfed group started 1 day later than the hypoxic group and received the amount of food consumed by the hypoxic group the day before. Feeding always took place between 15:00 and 16:00 hours. Body weight and food intake per day are shown in [20]. All experimental protocols were submitted to and approved by The Committee for Animal Care and Use of Maastricht University.

\section{RT-qPCR}

RNA was isolated using TRI REAGENT ${ }^{T M}$ (Sigma-Aldrich Chemie B.V., Zwijndrecht, NL). cDNA synthesis was performed with random hexamer primers using the Transcriptor First Strand cDNA Synthesis Kit (Roche Nederland B.V., Woerden, NL). Real-time quantitative PCR was performed in the iQ5 thermal cycler (Biorad, Hercules, CA, USA), using the qPCR SyBr Green Fluorescein Mix (Abgene, Epsom, UK) with intron-spanning primers (Table 2). mRNA expression was normalized to $18 \mathrm{~S}$ rRNA. 


\begin{tabular}{llll}
\hline \hline gene & NCBI & Forward primer (5'-3') & Reverse primer (5'-3') \\
\hline 18S & NR_003278.1 & AGTTAGCATGCCAGAGTCTGC & TGCATGGCCGTTCTTAGTTG \\
Murf1 (Trim63) & NM_00103904 & TGTCTGGAGGTCGTTTCCG & CTCGTCTTCGTGTTCCTTGC \\
Atrogin1 (Fbxo32) & NM_026346.2 & ACCGGCTACTGTGGAAGAGA & CCTTCCAGGAGAGAATGTGG \\
Nedd4 & NM_010890.3 & GCTGCCAAGAGCACACACCTG & CAACGCCATCAAAGCCCTGT \\
Bnip3 & NM_009760.4 & CCATGTCGCAGAGCGGG & GACGGAGGCTGGAACGC \\
Map1lc3B & NM_026160.4 & GAGCAGCACCCCACCAAGAT & CGTGGTCAGGCACCAGGAA \\
Atg5 (Apg5) & NM_053069.5 & TTGAATATGAAGGCACACCCC & CTCTTGAAATGTACTGTGATGTCCAA \\
Gr & NM_008173.3 & CGCCAAGTGATTGCCGC & TGTAGAAGGGTCATTTGGTCATCCA \\
Glul (Gs) & NM_008131.3 & GGCCATGCGGGAGGAGA & GGTGCCTCTTGCTCAGTTTGTCA \\
Mstr & NM_010834.2 & GGCCATGATCTTGCTGTAACCT & CGGCAGCACCGGGATT \\
Klf15 & NM_023184.3 & TGCAGCAAGATGTACACCAAGAG & ATCGCCGGTGCCTTGAC \\
Glut1 & NM_011400.3 & TGACCATCGCCCTGGCCT & GGACCAGGGCCTACTTCAAAG \\
VegfA & NM_009505.4 & CTGTACCTCCACCATGCCAAGT & TCGCTGGTAGACATCCATGAACT \\
Ca9 & NM_139305.2 & CAGGAGGCCTGGCAGTTTT & TTCTTCCAAATGGGACAGCAA \\
\hline
\end{tabular}

Table 2. Sequences of primers used for RT-qPCR to assess expression of the indicated genes.

\section{Determination of fiber cross-sectional area (FCSA) and fiber-type composi- tion.}

Seven $\mu \mathrm{m}$ cryosections were cut from the central region of each muscle and stained with antibodies against laminin (L9393, Sigma-Aldrich Chemie B.V) to visualize fiber circumferences. To discriminate individual fiber types, the following stainings were carried out simultaneously using antibodies against the respective myosin heavy chain (MyHC) isotypes: MyHC-IIA (333-7H1, gift from A.F.M. Moorman [21]) and MyHC-I (A4.840, Developmental Studies Hybridoma Bank (DSHB)) for soleus tissue sections, and MyHC-IIA with MyHC-IIB (BF-F3, DSHB) for EDL sections. The unstained muscle fibers were considered to be composed of the remaining fiber types. FCSA and fiber types were analyzed with the LUCIA 4.81 imaging software package (NIKON).

\section{Determination of capillary density (CD).}

Capillaries were stained with anti-CD31 (PECAM1) antibody (557355, BD Biosciences) in combination with anti-laminin and anti-MyHC-IIB for EDL or anti-MyHC-I for soleus analysis, respectively. Analyses were performed using the LUCIA 4.81 imaging software package (NIKON). Capillary density (CD) represents the number of capillary transections per $\mathrm{mm}^{2}$ of a section. The capillary perimeter $(\mathrm{CP}, \mu \mathrm{m})$ was determined from the outline of the CD31 staining. Capillary contacts $(\mathrm{CC})$ represent the number of capillary transections in direct contact (pixel to pixel) with a muscle fiber (distance $<2.2 \mu \mathrm{m}$, corresponding to the dimension of 2 pixels). The density of capillary contacts (CCD) represents the number of capillary contacts per $\mathrm{mm}^{2}$ of a section. FCSA/capillary contact was calculated by dividing the average FCSA of one fiber type with the average CC per picture. The interstitial space was determined on histologic sections using the ImageJ software. The interstitial space was defined as the remaining surface area after subtraction of all surface area covered by muscle fibers, by endo- and perimysium (laminin staining), by capillaries and larger blood vessels (CD31-staining) 
and nerves (perineurium detected by laminin staining).

\section{Western blotting.}

Soleus and EDL muscle tissue was lysed in presence of phosphatase- and protease inhibitors. Proteins were separated using gradient polyacrylamide gels and transferred to a nitrocellulose transfer membrane by electroblotting. The membrane was stained with Ponceau S solution to measure total protein loaded and subsequently blocked with non-fat dried milk, before incubating overnight with primary antibody (Table 3 ). Horse peroxidase-labeled secondary antibody was used in combination with chemiluminescence to visualize the protein bands. Bands were quantified and corrected for total amount of protein loaded, based on the Ponceau S staining.

\begin{tabular}{lll}
\hline Target & Product number & Company \\
\hline LC3B & 2775 & Cell Signaling \\
Poly-ubiquitin conjugates & BML-PW8805 & ENZO Life Sciences \\
GR S211 & 4161 & Cell Signaling \\
GR & 3660 & Cell Signaling \\
\hline
\end{tabular}

Table 3. Antibodies used for Western blotting.

\section{Statistics.}

Data are shown as means \pm SEM. Comparisons were computed with SPSS version 15 (SPSS Inc., IL, USA). Statistical significance was tested by ANOVA. The type of post-hoc analysis was based on data variance (Levene's test), with the Tukey test for data with equal variance, and the Games-Howell test for all other data. Statistical significance of basal (normoxic groups) differences between muscles was assessed using the Student's t-test. A P-value $<0.05$ was considered to be statistically significant and a $\mathrm{P}$-value $0.05 \leq \mathrm{P} \leq 0.1$ as indicating a trend.

\section{Results}

\section{Hypoxia-specific decrease of glycolytic muscle mass.}

Exposure to hypoxia affects food intake as we have shown previously (see Figure 2 in reference [20]). In our model, hypoxia-mediated decrease in food intake was maximal after 4 days of hypoxia and then increased to stabilize from day 11 onwards, albeit at a lower level than in normoxic animals [20]. We, therefore, included an extra group of mice, which were kept under normoxic conditions and pair-fed to the mice exposed to hypoxia. This allowed us to separate effects of hypoxia into a hypoxia-specific component (independent of food intake) and a component that can be mimicked by a reduction in food-intake. Accordingly, hypoxiaspecific responses refer to comparisons between pair-fed (PF) groups and mice 
exposed to hypoxia $(\mathrm{H})$, whereas the response to hypoxic conditions refers to the comparison between normoxic control $(\mathrm{N})$ and hypoxic mice and include the combined effects of hypoxia and reduced food intake. Compared to the pair-fed group, the EDL muscle of hypoxic mice already showed significant weight loss at 4 days, which was progressive during the next 2.5 weeks (Fig. 1A). The soleus, on the other hand, was less sensitive to hypoxia, but still showed a decrease to 93\% after 21 days when compared to normoxic animals $(H$ vs $N$ : $P<0.05$; Fig. 1B), but did not differ from the pair-fed mice, who showed a comparable trend $(\mathrm{H}$ vs $P F: P=0.10)$. These data reveal a hypoxia-specific reduction of tissue mass in the glycolytic EDL muscle only, whereas the weight reduction of the soleus was slower and partially mimicked by the reduction in food intake ( $N$ vs PF: $P=0.10$ ).
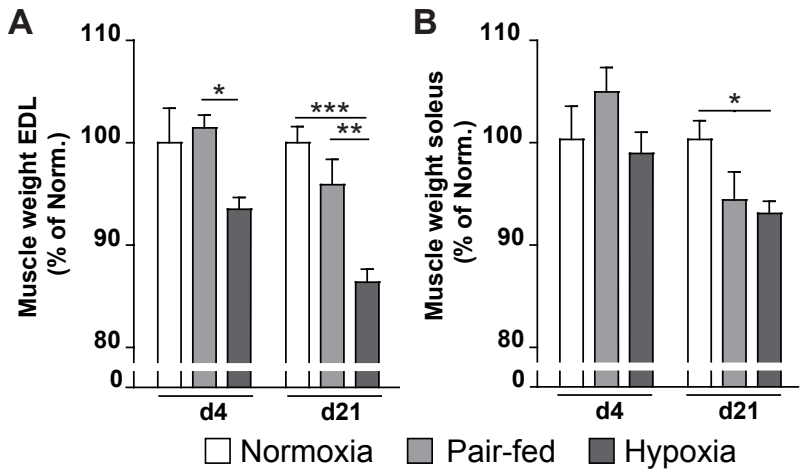

Figure 1. Hypoxia-specific decrease of glycolytic but not oxidative muscle weights.

Mice were exposed to normoxia $(\mathrm{N})$ or hypoxia $(\mathrm{H})$, and one normoxic group was pair-fed (PF) to the hypoxia group. Mice were sacrificed at the indicated time points and muscle weights were determined: (A) EDL (B) soleus. Muscle weights were corrected for starting body weight and expressed as the percentage of the normoxic value for the corresponding time point. Significant differences between groups at a given time point are indicated by asterisks, with ${ }^{*}=P<0.05,{ }^{* *}=P \leq 0.01$ or ${ }^{* *}=$ $P \leq 0.001$ ( $n=6-8$ per group).

\section{Hypoxia reduces the fiber cross-sectional area (FCSA) in the EDL only.}

Transverse sections of the EDL (Fig. 2A) and the soleus muscles (Fig. 2B) were stained with antibodies against myosin heavy chains and laminin to identify individual fiber types (left panels) and their contours (right panels). In the EDL a small but significant reduction of the number of type IIB fibers was observed after 21 days ( $\mathrm{H}$ vs $\mathrm{PF}: \mathrm{P}<0.01$ ) (Fig. $2 \mathrm{C}$ ), whereas in the soleus, the numbers of individual fibers were not changed under any of the experimental conditions (Fig. 2D). In the EDL, a significant reduction of the size of all fibers (Total) was already observed after 4 days of hypoxia, was progressive and had a clear hypoxiaspecific component ( $\mathrm{PF}$ vs $\mathrm{H}$ : $\mathrm{P}<0.05)$. 

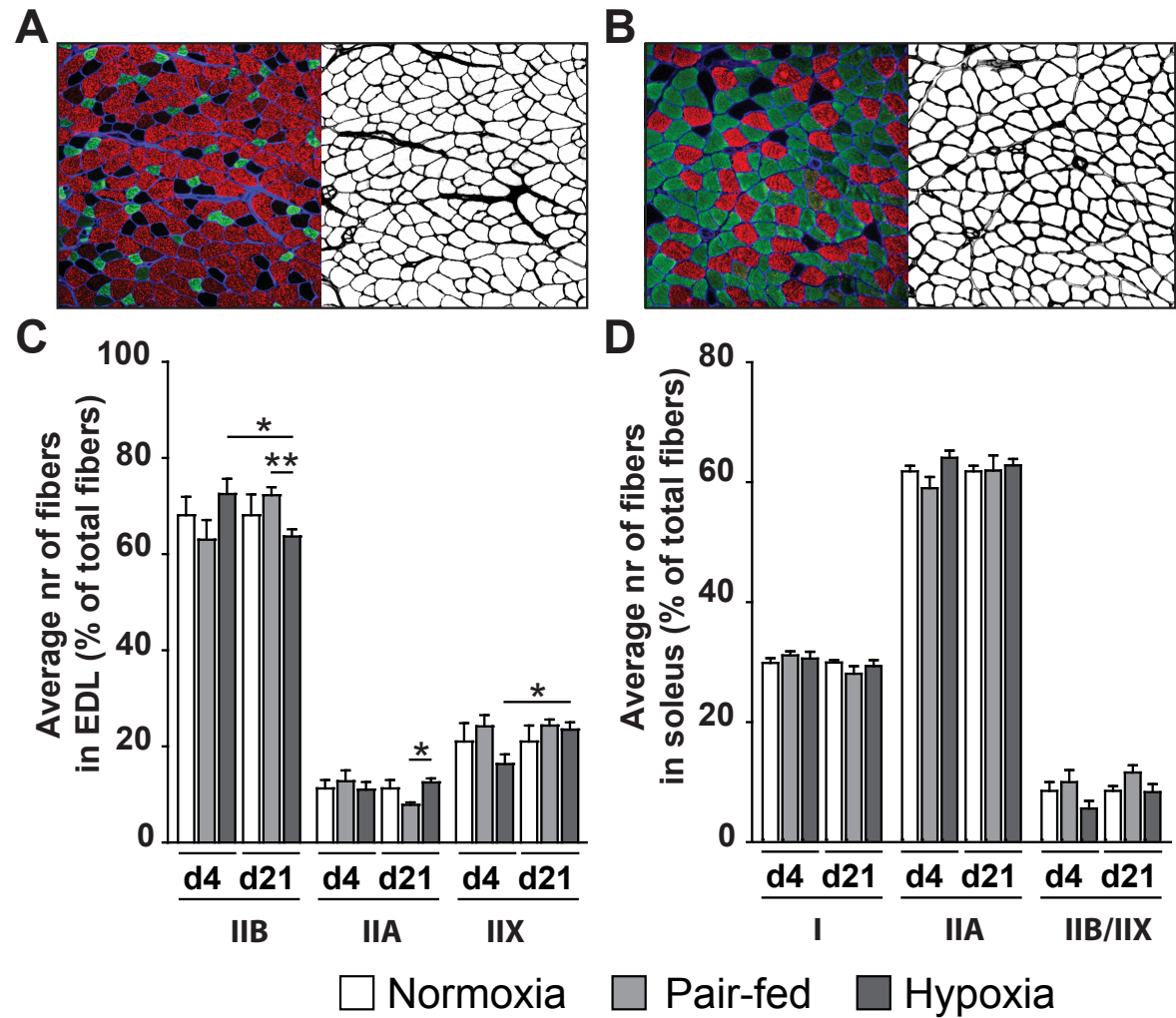

Figure 2. 21 days of hypoxia do not lead to a fiber type shift.

Muscle sections were analyzed histologically using fluorescence-labeled antibodies. (A) Left panel: immunofluorescence staining of a cross section of the EDL muscle at 21 days with antibodies against MyHC-IIA (green), MyHC-IIB (red), and laminin (blue) to visualize fiber boundaries. Unstained fibers represent MyHC-IIX fibers (black). Right panel: fiber boundaries and blood vessels of the same section visualized by anti-laminin staining. (B) Left panel: immunofluorescence staining of a cross section of the soleus muscle at 21 days with antibodies against MyHC-I (red), MyHC-IIA (green) and laminin (blue). Unstained fibers (black) represent MyHC-IIX/IIB fibers. Right panel: fiber boundaries and blood vessels of the same section visualized by anti-laminin staining. (C): Average number of fibers per fiber type expressed as percentage of total fibers in the soleus muscle at days 4 and 21 inthe three groups of mice. (B): Average number of fibers per fiber type expressed as percentage of total fibers in the EDL. Significant differences between groups at a given time point are indicated by asterisks, with ${ }^{*}=\mathrm{P}<0.05,{ }^{* *}=\mathrm{P} \leq 0.01$ or ${ }^{* * *}=\mathrm{P} \leq 0.001$ ( $\mathrm{n}=6-8$ per group). 
Analysis of the FCSA of individual fibers revealed that the changes were mostly due to a decrease in type IIB fibers, and a smaller decrease in type IIA fibers, whereas type IIX fibers were not affected (Fig. 3A). In the soleus, the mean FCSA of individual fiber types and accordingly of all fiber types (Total) was not significantly changed under any of the experimental conditions (Fig. 4A). Further analysis of the size distribution of specific fibers of the EDL revealed a small shift with the disappearance of the largest IIB- and IIA-type fibers. Small changes were already visible at day 4 (Fig. $3 \mathrm{C}$ and were more pronounced at day 21 (Fig. 3D). In the soleus, we did not find this decrease in size for any of the fiber types throughout the experiment (Fig. 4B, C).We did not observe a fiber-type shift after 4 or 21 days of hypoxia in either muscle. Since the cross-sectional area of the EDL is covered for $86 \%$ by the larger type IIB fibers with the remainder made up by the smaller type IIA and IIX fibers, respectively (Fig 3B), the reduction in muscle weight during hypoxia was mainly due to atrophy of type IIB fibers. These data show that the type IIB fibers in the EDL are most sensitive to hypoxia.

\section{Differential expression of HIF1 $\alpha$ target genes in EDL and soleus}

Previously, we demonstrated lowered $\mathrm{SaO} 2$ (saturation level of oxygen in hemoglobin) and $\mathrm{PaO} 2$ (partial pressure of oxygen in arterial blood) values in the blood of mice after acute ( 2 and 4 days) and chronic ( 21 days) exposure to hypoxia [20]. To assess hypoxia-associated signaling, expression of the HIF1aresponsive target genes Glut1 (glucose transporter 1, Fig. 5A), Vegfa (vascular endothelial growth factor A, Fig. 5B) and Ca-9 (carbonic anhydrase 9, Fig. 5C) was determined. Glut1 expression was most sensitive to hypoxia and Ca-9 least responsive. Basal expression levels of Glut1 and Vegfa were 1.4-fold higher in the soleus than the EDL, and Ca-9 3.1-fold higher in the EDL. Glut1 expression was induced in both muscles already at day $4(\sim 1.5$-fold, $\mathrm{H}$ vs $\mathrm{N})$. In the soleus, the increased expression was independent of food intake and similar under acute and chronic hypoxia, whereas in the EDL, the increase at day 4 was partially accounted for by reduced food intake. After 21 days, however, Glut 1 expression was further increased and hypoxia-specific in the EDL as well. Vegfa showed a hypoxia-specific increase in both muscles only after 21 days, with a trend (PF vs $\mathrm{H}, \mathrm{P}=0.09$ ) already visible in the soleus after 4 days. A significant, hypoxia-specific increase of $\mathrm{Ca}-9$ was only found in the EDL after 21 days. Together, these data suggest a hypoxic response in both muscles, with a stronger initial response in the oxidative soleus muscle and a stronger late response in the EDL. 

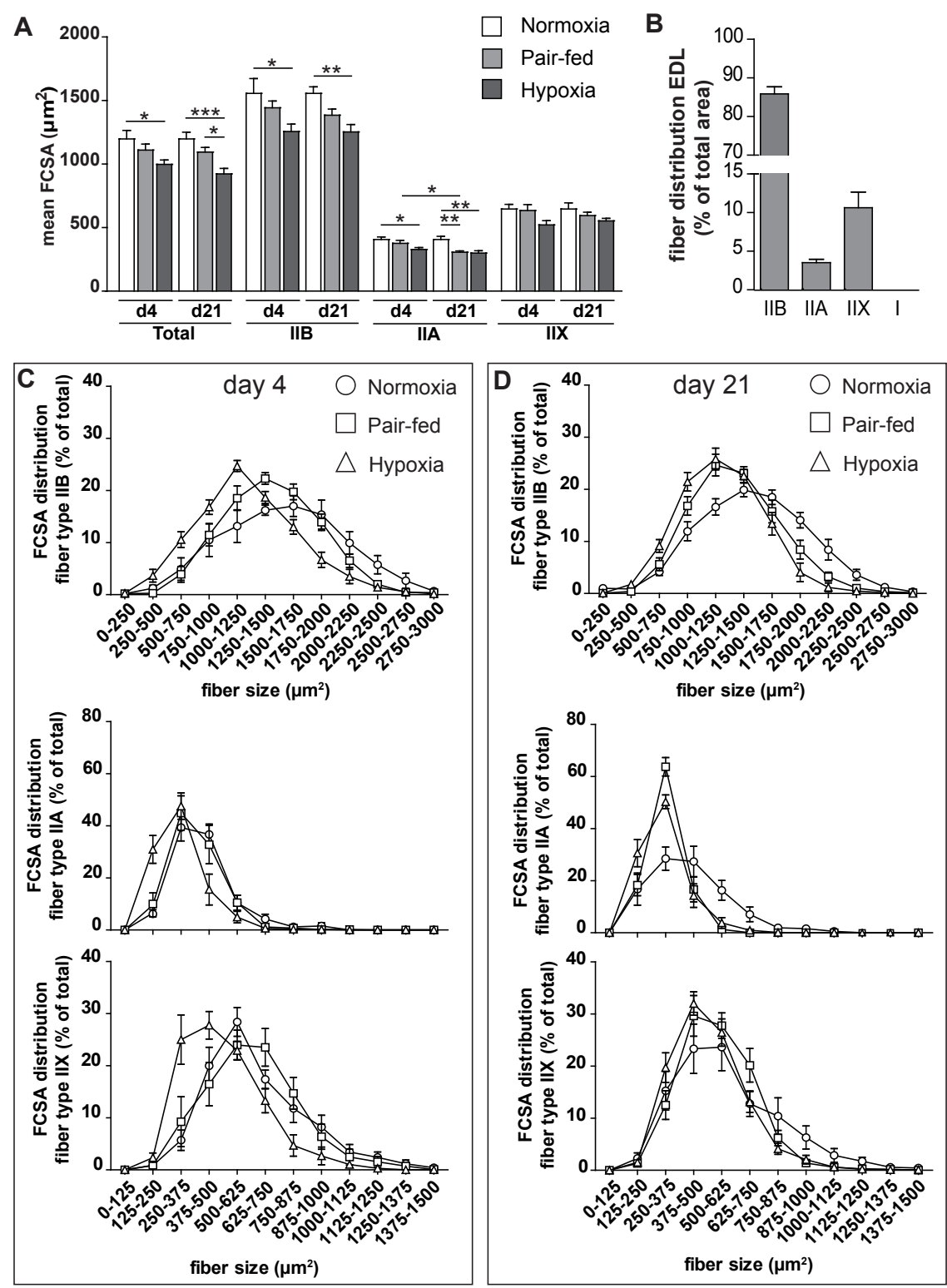

Figure 3. Hypoxia-specific decrease of the fiber cross-sectional area (FCSA) in the glycolytic EDL.

Muscle sections were analyzed histologically using fluorescence-labeled antibodies. (A) Mean FCSA of all fibers (Total) and individually stained fibers type IIB and IIA, and unstained fibers (type IIX) of the EDL muscle. (B) Fiber distribution in the EDL as percentage of the total surface area. (C) FCSA distribution of the EDL muscle per fiber type on day 4, and (D) on day 21; no significance indicated; top panels: type IIB fibers, middle panels: type IIA fibers, bottom panels: type IIX fibers. Significant differences between groups at a given time point are indicated by asterisks, with ${ }^{*}=P<0.05,{ }^{* *}=P \leq$ 0.01 or $^{* * *}=\mathrm{P} \leq 0.001$ ( $\mathrm{n}=6-8$ per group). 

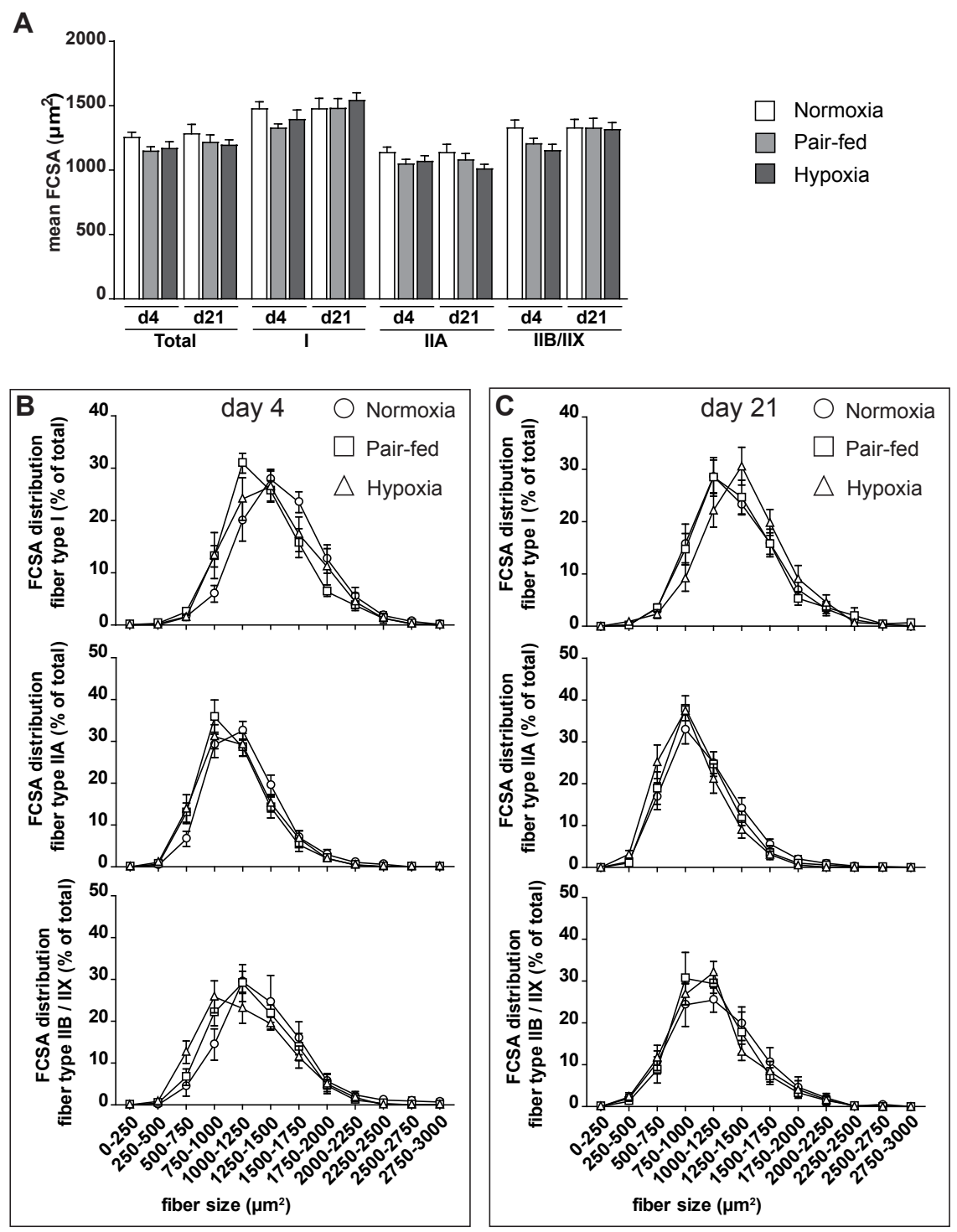

Figure 4. The oxidative soleus muscle shows no changes in fiber size distribution.

Muscle sections were analyzed histologically using fluorescence-labeled antibodies. (A) Mean FCSA of all fibers (Total) and individually stained fibers (type IIB/IIX) of the soleus muscle. (B) FCSA distribution of the soleus muscle per fiber type on day 4, and (C) on day 21; no significance indicated; top panels: type I fibers, middle panels: type IIA fibers, bottom panels: type IIB/X fibers Significant differences between groups at a given time point are indicated by asterisks, with ${ }^{*}=P<0.05,{ }^{*}=P \leq$ 0.01 or ${ }^{* * *}=P \leq 0.001$ ( $n=6-8$ per group). 

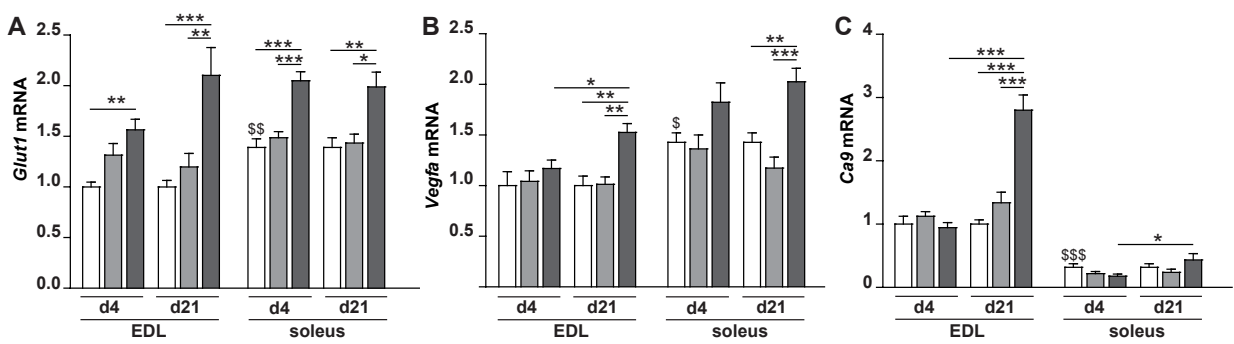

$\square$ Normoxia $\square$ Pair-fed $\square$ Hypoxia

Figure 5. Differential expression of HIF1a target genes.

mRNA expression of HIF1a target genes was determined. (A): Glut1, (B): Vegfa, (C): Ca-9. mRNA concentration was corrected for 18S RNA concentration and normalized to the normoxic value of the EDL at day 4. Significant differences of normoxia (basal) values between muscles are indicated with dollar signs (\$) and significant differences between groups at a given time point are indicated by asterisks, with ${ }^{*} / \$=\mathrm{P}<0.05,{ }^{* *} / \$=\mathrm{P} \leq 0.01$ or ${ }^{* * *} / \$ \$ \$=\mathrm{P} \leq 0.001$ ( $\mathrm{n}=6-8$ per group).

\section{Gene expression of ubiquitin ligases shows differential sensitivity to hypoxia.}

To assess whether oxidative and glycolytic muscles showed differential activation of UPS-mediated protein degradation pathways, we measured the expression of the E3 ubiquitin ligases Murf1, Atrogin-1 and Nedd4 (Fig. 6A-C) and determined the amount of poly-ubiquitin conjugates in muscle (Fig. 6D, E). Basal expression of Atrogin-1 and Murf1 was comparable in EDL and soleus and both muscles responded with increased expression under hypoxia, but the response in the EDL was larger for both genes and faster for Atrogin-1. Increased expression of Murf1 could be mimicked by a reduction in food intake in both muscles, whereas the increased Atrogin-1 expression showed a clear hypoxia-specific component, but only in the EDL. Basal Nedd4 expression was almost 5 times higher in the EDL than the soleus and was further increased in a hypoxia-specific manner in the EDL. These data show that Nedd4 was most sensitive to hypoxia-specific induction, whereas induction of both Murf1 and Atrogin-1 also showed a response to the decreased food intake. The increased gene expression of ubiquitin ligases was not reflected in an increase of poly-ubiquitin conjugates in either the EDL or soleus.

\section{Effects of hypoxia on the autophagy-lysosome pathway (ALP).}

To investigate ALP-mediated protein degradation, the expression of Map1/c3b, Bnip3 and Atg5 was measured (Fig. 7A-C), while LC3B activation was determined by quantifying lipidated LC3B-II (Fig. 7D,E). Basal expression of Map1/c3b was similar in EDL and soleus, and both muscles showed increased expression after acute and chronic hypoxia. In the soleus, this increase dependent on food restriction, whereas the more pronounced increase in the EDL contained a hypoxiaspecific element. Basal expression of Bnip3 was 2-fold higher in the soleus, but 
both muscles showed a hypoxia-specific upregulation. In the EDL, this was partly due to the hypoxia-mediated reduction in food intake. Basal Atg5 expression was slightly higher in the soleus, but hypoxia-specific increased expression was only observed in the EDL. Induction of Atg 5 expression was hypoxia-specific, whereas Map1/c3b and Bnip3 showed also a response to the lowered food intake. LC3B protein concentration was $\sim 2$-fold and that of the active lapidated form, LC3B-II, even 3.2-fold higher in the soleus than the EDL. Hypoxia led to a hypoxia-specific reduction of LC3B-II in both soleus and EDL in the acute phase. In the chronic phase the reduction also showed a food-intake dependent component.
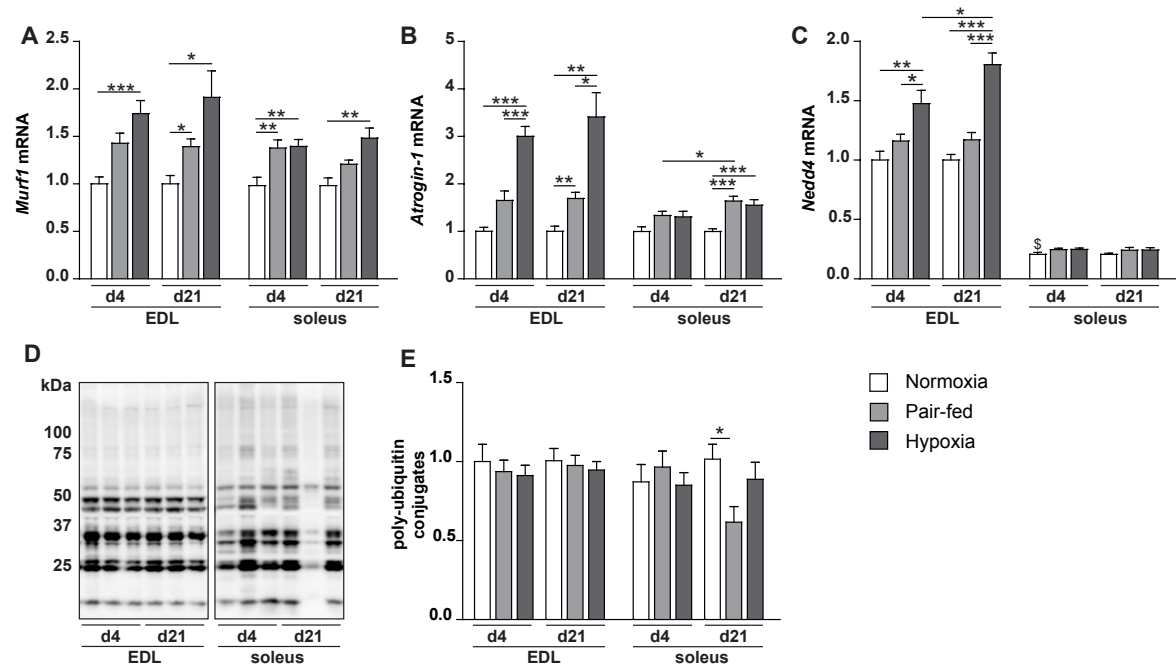

Figure 6. Hypoxia affects the expression of genes of the ubiquitin-proteasome system (UPS) predominantly in the glycolytic EDL muscle but does not lead to accumulation of poly-ubiquitin conjugates.

mRNA expression of genes belonging to the ubiquitin-proteasome system (UPS). (A): Murf1, (B): Atrogin-1, (C): Nedd4, mRNA concentration was corrected for 18S RNA and normalized to the normoxic value of the EDL at day 4. (D): Representative Western blots of poly-ubiquitin conjugates in protein extracts of EDL (left panel) and soleus muscles (right panel) from mice of all three experimental groups at 4 and 21 days. (E) Total poly-ubiquitin conjugates per condition. Ponceau $\mathrm{S}$ staining was used to correct for protein loading. Significant differences of normoxia (basal) values between muscles are indicated with dollar signs $(\$)$. Significant differences between groups at a given time point are indicated by asterisks, with ${ }^{*} \$=P<0.05,{ }^{* *} / \$ \$=P \leq 0.01$ or ${ }^{* *} / \$ \$ \$=P \leq 0.001$ ( $n=6-8$ per group) 

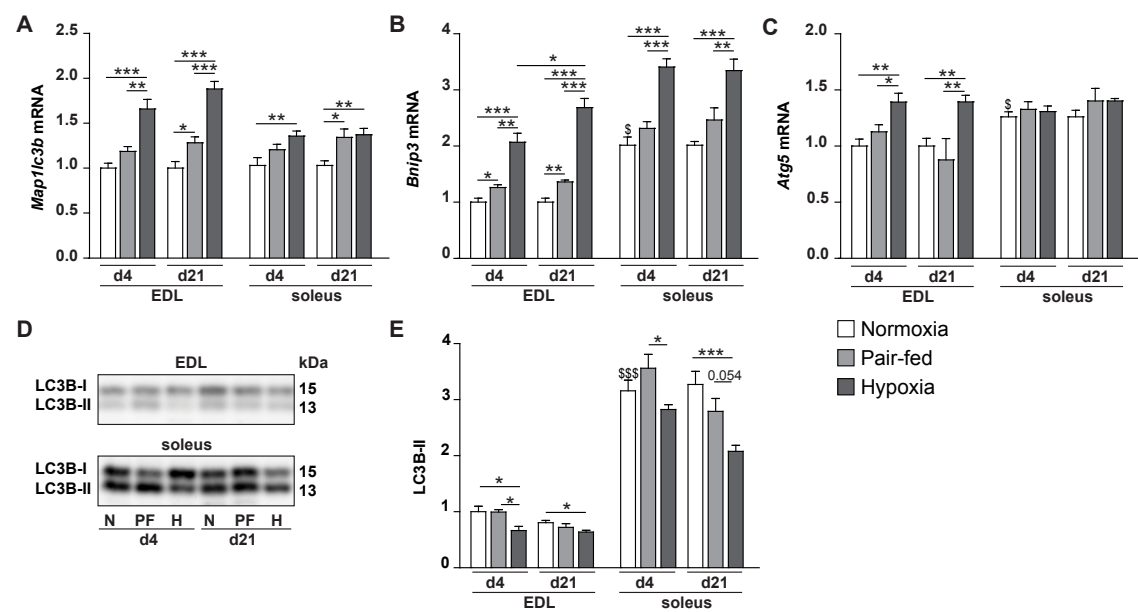

$$
\begin{aligned}
& \square \text { Normoxia } \\
& \square \text { Pair-fed } \\
& \square \text { Hypoxia }
\end{aligned}
$$

Figure 7. Hypoxia-specific induction of genes of the ALP in the EDL.

mRNA expression of genes belonging to the autophagy-lysosome pathway (ALP) was measured: $(A)$ : Map1/c3b, (B): Bnip3, (C): Atg5. mRNA concentrations were corrected for 18S RNA and normalized to the normoxic value of the EDL at day 4. (D): Representative Western blots of LC3B protein in the EDL (upper panel) and soleus muscles (lower panel) from mice of all three experimental groups at 4 and 21 days. (E) LC3B-II concentration normalized to the normoxic value of the EDL at day 4. PonceauS staining was used to correct for protein loading. Significant differences of normoxia (basal) values between muscles are indicated with dollar signs (\$). Significant differences between groups at a given time point are indicated by asterisks, with ${ }^{*} / \$=P<0.05,{ }^{* *} / \$ \$=P \leq 0.01$ or ${ }^{* * *} / \$ \$ \$=P \leq 0.001$ ( $n=6-8$ per group).

\section{Hypoxia-induced glucocorticoid signaling is more prominent in the glycolytic EDL.}

To assess whether glucocorticoid signaling was involved in hypoxia-mediated muscle atrophy, we analyzed GR content and phosphorylation at Ser211 (Figs. $8 \mathrm{~A}-\mathrm{C}$ ) and mRNA expression (Fig. 8D) and that of its target genes Krüppel-like factor 15 (Klf15), myostatin (Mstn) and glutamine synthetase (Glul) (Figs. 8E-G). Except for a temporary hypoxia-specific decrease of phosphorylated GR in the EDL, changes in GR content and phosphorylation at Ser211 were small. Basal mRNA concentrations of $\mathrm{Gr}$ were comparable in both muscles and showed a hypoxia-specific increase in expression within 4 days, which was, however, only sustained in the EDL. Basal expression of the GR target gene Klf15 was comparable in both muscles and both responded with increased expression to acute and chronic hypoxia. In the soleus, however, this response was dependent on the reduced food intake, whereas in the EDL it was partially hypoxia-specific (Fig. $8 \mathrm{E}$ ). Basal expression of Mstn was 27 -fold higher in the EDL than the soleus (Fig. $8 \mathrm{~F}$ ). Whereas expression was insensitive to hypoxia in the soleus, expression was increased in the EDL after 21 days, which was partially accounted for by reduced food intake. Basal expression of Glul was 2.3-fold higher in the EDL than 
the soleus (Fig. 8G). This expression was increased almost 2-fold in a hypoxiaspecific manner at day 21 in the $E D L$, whereas only a trend ( $H$ vs $N, P=0.06$ ) towards an increased expression was seen in the soleus. To summarize, expression of Gr and Glul were upregulated in a hypoxia-specific manner, whereas KIf15 was regulated by food intake. The glycolytic EDL muscle was more sensitive to hypoxia-specific induction of glucocorticoid signaling than the soleus.

A

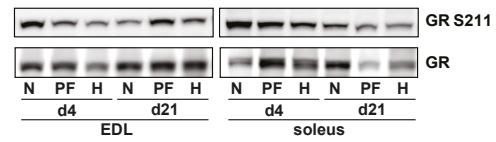

$\square$ Normoxia $\square$ Pair-fed $\square$ Hypoxia

B

C
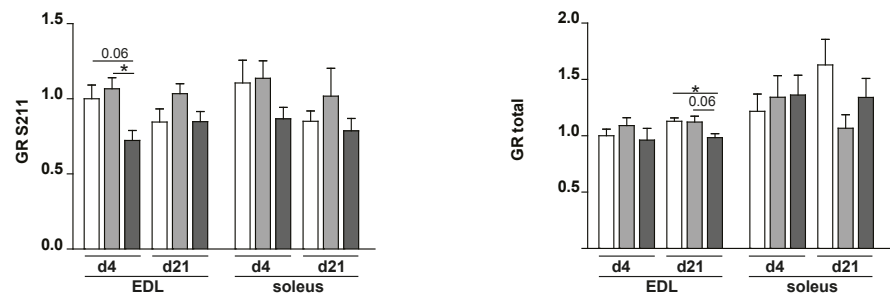

D 2.0

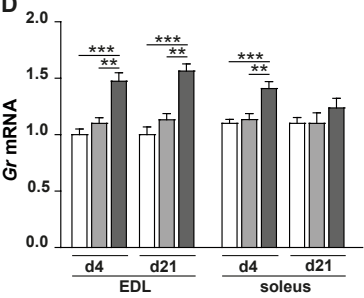

E
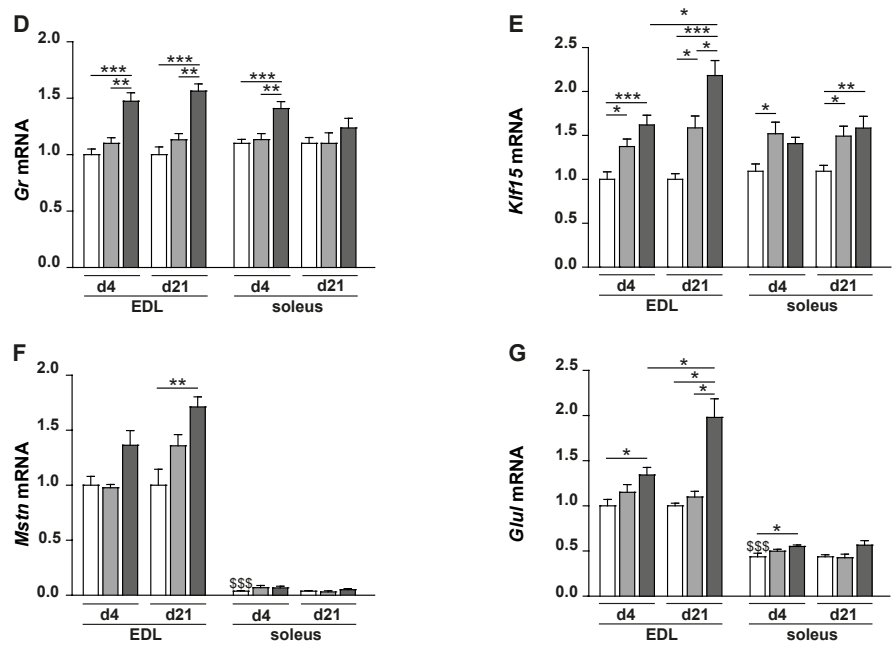

Figure 8. Hypoxia-induced glucocorticoid signaling is more prominent in the glycolytic EDL muscle.

Phosphorylation of GR at serine 211 was measured as an indication of its activation in the EDL and the soleus of mice exposed to one of the 3 experimental conditions (normoxia, pair-fed or hypoxia) for 4 or 21 days. (A): representative Western blots of total GR and phosphorylated GR. (B) GR Ser211, (C): total GR. Ponceau S staining of Western blots was used to correct for protein loading. mRNA expression of $\mathrm{Gr}$ and its target genes was determined. (D): $\mathrm{Gr}$, (E): Klf15, (F): Mstn, (G): Glul. mRNA concentration was corrected for 18S RNA and normalized to the normoxic value of the EDL at day 4. Significant differences of normoxia (basal) values between muscles are indicated with dollar signs (\$). Significant differences between groups at a given time point are indicated by asterisks, with * $/ \$=P<$ $0.05,{ }^{* *} / \$ \$=P \leq 0.01$ or ${ }^{* * *} / \$ \$ \$=P \leq 0.001$. ( $n=6-8$ per group). 


\section{Hypoxia results in muscle specific adaptions of the capillary network.}

Hypoxia triggers angiogenesis [22-25]. We used CD31 staining to identify and quantify capillaries in the EDL and the soleus (Figs. 9A-B). The increased Vegfa expression suggests adaptations of the capillary network within the muscles. Under normoxic conditions, the capillary density (CD) in the EDL (Fig. 9C) was significantly lower than in the soleus (807 vs 1079 capillaries/mm2, respectively). The number of capillaries/mm2 (Fig. 9C) and of capillary contacts/mm2 (Fig. 9D) were both 1.4-fold higher in the soleus than the EDL. Capillaries were also slightly larger in the soleus (Fig. 9E), and a hypoxia-specific reduction of the interstitial space between fibers was found in the soleus after 21 days (Fig. 9G). Together, these changes explain the more frequent capillary contacts in this muscle, resulting in a smaller FCSA per capillary contact (Fig. 9F), which may facilitate a better blood supply to the muscle fibers of the soleus. In the EDL, a hypoxia-specific increase in capillary density was observed, but no change in the capillary contacts per unit surface. In both muscles, hypoxia, therefore, caused a decrease of the FSCA per capillary contact and thereby a better blood supply of the fibers. The effects of reduced food intake on capillary density, contacts and perimeter were small.
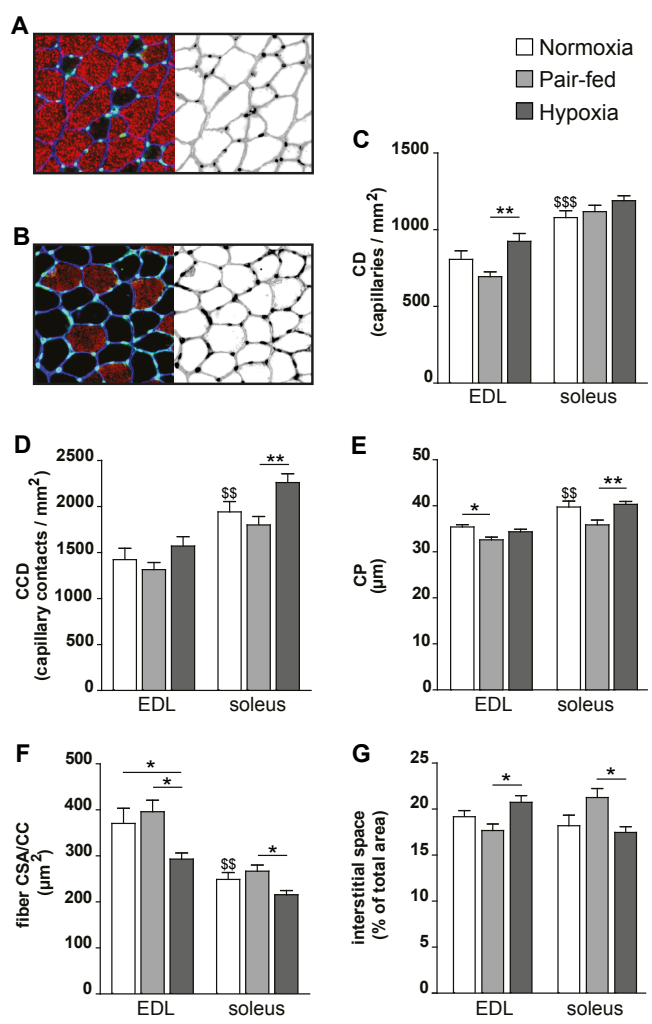

Figure 9. Hypoxia results in muscle specific adaptions of the capillary network and the interstitial space.

Capillary staining with fluorescent antibodies was combined with fiber type-specific staining to characterize the capillary network around fibers. Laminin-staining was used to indicate fiber boundaries. Immunofluorescence staining of a cross section of the (A) left panel: EDL with antibodies against CD31 (green), MyHC-IIB (red), and laminin (blue); unstained fibers (black) represent MyHC-IIA/IIX fibers; and (B) left panel: soleus muscle with antibodies against CD31 (green), MyHC-I (red), and laminin (blue); unstained fibers (black) represent MyHC-IIA/IIB/IIX fibers. (A, B) right panels: fiber boundaries of the same section visualized by anti-laminin (gray) and CD31 staining (black). (C): Capillary density CD (capillaries/mm2), (D): capillary contacts per $\mathrm{mm} 2$ section $C C D,(E)$ : capillary perimeter $\mathrm{CP}(\mu \mathrm{m}),(F)$ : interstitial space between fibers $(\mathrm{G})$ : FCSA per capillary contact $(\mu \mathrm{m} 2)$. Significant differences of normoxia (basal) values between muscles are indicated with dollar signs $(\$)$. Significant differences between groups at a given time point are indicated by asterisks, with $* / \$=P<0.05$, ${ }^{* *} / \$ \$=P \leq 0.01$ or ${ }^{* * *} / \$ \$ \$=P \leq 0.001(n=6-8$ per group). 


\section{Discussion}

In this study we investigated the atrophy responses of a mainly oxidative muscle, the soleus, and a predominantly glycolytic muscle, the EDL. The effects of hypoxia on muscle consisted of a food intake-dependent effect and a hypoxiaspecific effect (independent of the hypoxia-mediated reduction in food intake). The main findings were that 1 ) both muscles adapted to chronic hypoxia with a similar result, namely a reduction of the FCSA per capillary contact, but achieved this via a different path, that is a decrease of FCSA in the EDL and an increase of capillary contacts in the soleus; 2 ) the increased sensitivity of the EDL to hypoxia was reflected in increased hypoxia-specific expression of genes of the UPS and the ALP, whereas in the soleus this response was smaller and showed a stronger dependence on food intake; 3) expression of Gr mRNA and target genes of GR was hypoxia-specific in the EDL and stronger than in the soleus; 4) the ubiquitin ligase Nedd4 and the autophagy-related factor Atg5 are upregulated in a hypoxia-specific manner.

In the present study, we showed a rapid decrease of muscle mass and fiber cross-sectional area in the glycolytic EDL muscle, but not the oxidative soleus muscle in response to normobaric hypoxia. After 3 weeks of hypoxia, muscle atrophy was still most prominent in the EDL, suggesting that loss of glycolytic muscle mass is a feature of chronic hypoxia. The reduction in muscle mass was reflected in a reduction of the FCSA of the predominant type IIB muscle fibers in the EDL. Rats that were exposed for 10 weeks to hypobaric hypoxia also showed a selective reduction of the FCSA of the glycolytic EDL [26]. A consistent finding in COPD patients is a fiber type redistribution from oxidative type I fibers towards more glycolytic type II fibers [11-14, 27], which may already be present in mild to moderate disease [28]. We previously showed selective atrophy of type II fibers in COPD patients relative to age-matched control subjects [10]. The findings of the present study suggest that this may be due to an unfortunate series of events - initiated by a shift towards type II fibers, that is, towards a muscle phenotype that subsequently renders COPD patients more sensitive to disease-related episodes of hypoxia and cachexia in advanced disease.

Activation of GR signaling in muscle induces atrophy [29, 30]. Sensitivity of fast-twitch muscle fibers, like the EDL, to glucocorticoid-induced atrophy was previously demonstrated in rats [31]. Here, we show that basal Gr mRNA concentrations were the same in both muscles, but that acute and chronic hypoxia upregulated $\mathrm{Gr}$ expression only in the glycolytic EDL muscle. Interestingly, GR protein levels did not respond accordingly. However, GR recycling and degradation are tightly controlled following GR activation, and may have been affected by 
hypoxia [32]. It was shown previously that severe hypoxia potentiates glucocorticoid activity through the induction of the glucocorticoid receptor in human kidney cells, and that HIF1 $\alpha$ is responsible for at least part of this induction [33]. The observed predominant GR response to hypoxia in the EDL is in line with a higher sensitivity of this glycolytic muscle to glucocorticoids [34]. Klf15 is a GR-sensitive gene required for amino acid degradation to provide carbohydrate backbones for gluconeogenesis under fasting conditions [35]. In line with this function, increased expression was observed in soleus and EDL muscles in response to reduced food intake. KLF15, glutamine synthetase (GLUL) and myostatin have been implicated in protein catabolism and inhibition of muscle growth, respectively [36, 37]. The additional hypoxia-induced increase of their mRNAs in the EDL corresponds with the accentuated hypoxia-specific loss of mass of this muscle.

The observed hypoxia-induced skeletal muscle atrophy involves increased expression of genes of the UPS and ALP-mediated protein degradation pathways [20]. During UPS-mediated protein degradation, proteins are labeled through the addition of ubiquitin chains by E3 ubiquitin ligases. The lack of accumulation of poly-ubiquitin conjugates indicates that proteasomal capacity is not limiting and ubiquitin homeostasis is not disturbed [38]. Basal expression and induction by hypoxia-induced reduction of food intake of the E3 ubiquitin ligases Murf1 and Atrogin-1 was not different between muscle types. Hypoxia-specific, food intakeindependent increases in E3 ubiquitin ligase expression were only observed in the glycolytic EDL muscle. Interestingly, changes in KIf15 expression paralleled the response of Murf1 and Atrogin-1, both downstream targets of KLF15 [37]. The increased expression of Murf1 and Atrogin-1 may be the consequence of an increased sensitivity of the EDL to corticosterone and GR signaling. Expression of the E3 ubiquitin ligase Nedd4 did not mimic the expression patterns of Murf1 and Atrogin-1. This ligase is highly expressed in the glycolytic EDL muscle but not the oxidative soleus muscle. In addition, increased Nedd4 expression is dependent on hypoxia only and not on reduced food-intake. Increased Nedd4 expression was previously identified as a marker to distinguish muscle atrophy caused by reduced muscle tension from cachexia-induced atrophy [39]. Our findings indicate that Nedd4 expression may also be a marker for hypoxia and hypoxia-induced atrophy in glycolytic muscle.

The elevated expression of Bnip3, Map1/c3B and Atg5 in the hypoxic EDL suggested that the ALP is involved in hypoxia-specific atrophy of this muscle. In addition, expression of Bnip3, a hypoxia-inducible member of the Bcl-2 family and a regulator of cardiomyocyte mitophagy under hypoxic conditions [40], was increased in the oxidative soleus muscle by hypoxia, and this was independent 
of food intake. Bnip3 may therefore contribute to muscle adaptations other than atrophy, such as mitochondrial loss by mitophagy [41, 42]. The increased hypoxia-specific expression of Maplc3b and Atg5 in the EDL together with the decreased concentration of LC3B-II may be interpreted as increased flux through the autophagy-lysosome pathway [43]. It has to be noted, however, that relations between LC3B-I and LC3B-II depend on the type of cell, and the type of stress and cell-specific transcriptional regulation, rendering the interpretation of these data notoriously difficult [43]. Although the main objective of this study was to characterize the different sensitivities of oxidative and glycolytic muscle towards hypoxia und underlying mechanisms, it seems worthwhile to note that the expression of Atg5, a crucial component of the autophagy machinery, is entirely dependent on hypoxia and independent of food intake, so that it qualifies, like Nedd4, as a marker for hypoxia-induced atrophy in glycolytic muscle.

Glycolytic muscles in mice have higher concentrations of HIF1 $\alpha$ protein and mRNA than oxidative muscles [44]. HIF1 $\alpha$ activation can regulate the expression of its downstream targets Ca-9, Vegfa and Glut1, as well as Bnip3. Expression of Glut1 and Vegfa increased in both muscles upon exposure to hypoxia, whereas Ca-9 was only upregulated in the glycolytic EDL muscle and only after chronic exposure. This latter finding suggests a delayed response to hypoxia for this gene, although the exact function of $\mathrm{Ca}-9$ remains unclear. The early increase in glucose transporter 1 (Glut1) expression probably reflects the increased glucose uptake under hypoxia as shown by others [45]. Hypoxia indeed resulted in lowered blood glucose concentrations in the morning after 21 days (Fig. 10).

VEGF is a potent angiogenic factor in hypoxic tissues [46]. Basal Vegfa expression was higher in oxidative soleus than in glycolytic EDL muscle. This may relate to the higher capillary density in oxidative muscles, as expression of Vegfa in endothelial cells is 2-fold higher than in skeletal muscle cells [47]. Chronic hypoxia resulted in a significantly increased expression of Vegfa in both muscles. The angiostatic effect of glucocorticoids has been explained by their inhibition of Vegfa expression [48], which at least in vitro, is potentiated by hypoxia [33]. It is, therefore, tempting to speculate that the effects of increased Vegfa expression were attenuated in glycolytic muscles like the EDL by their higher sensitivity to increased circulating corticosterone concentrations. Such an attenuated angiogenic response may well account for the poor adaptation of the capillary structure to hypoxia in glycolytic muscles. The capillary density was lower in glycolytic EDL than in oxidative soleus muscle confirming earlier studies [49]. The resulting larger fiber cross-sectional area (FCSA) per capillary further predisposes the glycolytic EDL muscle to oxygen and nutrient deficiency. The hypoxia-induced 
reduction in muscle fiber diameter in the EDL may have partically compensated for the cited increases in sensitivity to hypoxia. In the oxidative soleus muscle, hypoxic conditions increased the number of capillary contacts per muscle fiber $[46,50]$, which reduces its sensitivity to hypoxia. The deep, more oxidative region of the plantaris muscle in rats also responded with an increase in capillarity to chronic hypoxia, whereas the superficial, more glycolytic region of the same muscle did not [51]. These findings reveal two distinct structural changes to adapt to hypoxia, that is, via a decrease in fiber diameter (EDL) or an increase in capillary contacts per fiber. In patients with moderate to severe COPD, no difference of FCSA per capillary contact was observed, although the number of capillaries per fiber was decreased compared to controls, except for type IIB fibers [12]. The loss of capillaries may be a long-term adaptive mechanism following the reduced FCSA observed in our relatively short-term study.

Increased protein turnover plays a role in muscle homeostasis under stress and does not necessarily result in severe muscle atrophy as long as the size of the stress falls within the adaptive capacity of the organism or cell. We showed in our previous article [20] that hypoxia stimulated both protein synthesis and degradation pathways. Furthermore, we showed that hypoxia activated the UPR without completely blocking mRNA translation. This may explain why the effects of severe hypoxia are relatively small in otherwise healthy mice. Similarly, it was recently shown that ER-stress and hypoxia-response pathways potentiated HIF1 transcriptional activity of targets like VEGF [52] thereby promoting adaptation. In COPD patients, however, where stress due to multiple stressors probably exceeds the adaptive capacity, certainly in the long run, the outcome is muscle atrophy.

\section{Limitations of the study}

We have not analyzed the effects of hypoxia-mediated inactivity, which may also contribute to muscle atrophy. We cannot exclude that hypoxia-associated inactivity contributes to the hypoxia-specific reduction in FCSA of IIB fibers found in our study. However, muscle unloading in rodents usually results in type I fiber atrophy. It has been shown that short-term hypoxia (10\% O2 for 2.5 days) resulted in clearly decreased activity levels [53], but the effects of chronic hypoxia on activity were not investigated in that study. Our hypoxic mice seemed drowsy during the first week of the experiment, but we noticed no obvious differences in activity with the normoxic group at later time-points. 


\section{Conclusion}

This study shows that hypoxia-induced muscle atrophy is more prominent in the glycolytic EDL than the oxidative soleus muscle. The atrophy observed in the EDL contained a hypoxia-specific effect that was independent of food intake, which involved activation of the catabolic proteasomal and lysosomal protein degradation pathways as well as increased expression of the glucocorticoid receptor gene and its target genes. Both muscles adaptated to hypoxia by a decrease of the fiber volume supported by one capillary contact, but this was achieved by a decrease in muscle fiber size in the EDL and an increase in the number of capillary contacts in the soleus muscle. The more pronounced atrophic response of glycolytic fibers in mice suggests that COPD patients may be more susceptible to hypoxia-induced muscle atrophy because of the predominance of glycolytic fibers in their muscles.

\section{Acknowledgements}

The authors gratefully acknowledge the technical support provided by: Els Terwindt, Paul van Dijk, Greet Mommen, Patricia Niedoba, Marleen Hendriks and Aafke Duisters.

\section{Grants and Disclosures}

This study was performed within the framework of the Dutch Top Institute Pharma, project T1-201. Partners in this project are Maastricht University Medical Center+, University Medical Center Groningen, University Medical Center Utrecht, GlaxoSmithKline, AstraZeneca, Nycomed and Danone Research. 


\section{References}

1. Wouters EF. Nutrition and metabolism in COPD. Chest. 2000;117(5 Suppl 1):274S-80S.

2. Mador MJ, Bozkanat E. Skeletal muscle dysfunction in chronic obstructive pulmonary disease. Respir Res. 2001;2(4):216-24.

3. Decramer M, De Benedetto F, Del Ponte A, Marinari S. Systemic effects of COPD. Respiratory medicine. 2005;99 Suppl B:S3-10.

4. Lainscak M, von Haehling S, Doehner W, Sarc I, Jeric T, Ziherl K, et al. Body mass index and prognosis in patients hospitalized with acute exacerbation of chronic obstructive pulmonary disease. Journal Cachexia Sarcopenia Muscle. 2011;2(2):81-6.

5. Batt J, Ahmed SS, Correa J, Bain A, Granton J. Skeletal muscle dysfunction in idiopathic pulmonary arterial hypertension. American journal of respiratory cell and molecular biology. 2014;50(1):74-86.

6. Herridge MS, Cheung AM, Tansey CM, Matte-Martyn A, Diaz-Granados N, Al-Saidi F, et al. One-year outcomes in survivors of the acute respiratory distress syndrome. The New England journal of medicine. 2003;348(8):683-93.

7. Gray-Donald K, Gibbons L, Shapiro SH, Macklem PT, Martin JG. Nutritional status and mortality in chronic obstructive pulmonary disease. American journal of respiratory and critical care medicine. 1996;153(3):961-6.

8. Boutellier U, Howald H, di Prampero PE, Giezendanner D, Cerretelli P. Human muscle adaptations to chronic hypoxia. Prog Clin Biol Res. 1983;136:273-85.

9. Soguel Schenkel N, Burdet L, de Muralt B, Fitting JW. Oxygen saturation during daily activities in chronic obstructive pulmonary disease. The European respiratory journal. 1996;9(12):25849.

10. Gosker HR, Engelen MPKJ, van Mameren H, van Dijk PJ, van der Vusse GJ, Wouters EFM, et al. Muscle fiber type IIX atrophy is involved in the loss of fat-free mass in chronic obstructive pulmonary disease. Am J Clin Nutr. 2002;76:113-9.

11. Natanek SA, Riddoch-Contreras J, Marsh GS, Hopkinson NS, Moxham J, Man WD, et al. MuRF-1 and Atrogin-1 Protein Expression and Quadriceps Fiber Size and Muscle Mass in Stable Patients with COPD. COPD. 2013;10(5):618-24.

12. Jobin J, Maltais F, Doyon JF, LeBlanc P, Simard PM, Simard AA, et al. Chronic obstructive pulmonary disease: capillarity and fiber-type characteristics of skeletal muscle. J Cardiopulm Rehabil. 1998;18(6):432-7.

13. Gosker HR, van Mameren H, van Dijk PJ, Engelen MP, van der Vusse GJ, Wouters EF, et al. Skeletal muscle fibre-type shifting and metabolic profile in patients with chronic obstructive pulmonary disease. The European respiratory journal. 2002;19(4):617-25.

14. Pereira MC, Isayama RN, Seabra JC, Campos GE, Paschoal IA. Distribution and morphometry of skeletal muscle fibers in patients with chronic obstructive pulmonary disease and chronic hypoxemia. Muscle Nerve. 2004;30(6):796-8. 
15. Eliason G, Abdel-Halim SM, Piehl-Aulin K, Kadi F. Alterations in the muscle-to-capillary interface in patients with different degrees of chronic obstructive pulmonary disease. Respir Res. 2010;11:97.

16. Mendias CL, Marcin JE, Calerdon DR, Faulkner JA. Contractile properties of EDL and soleus muscles of myostatin-deficient mice. J Appl Physiol 2006;101(3):898-905.

17. Macpherson PC, Wang X, Goldman D. Myogenin regulates denervation-dependent muscle atrophy in mouse soleus muscle. J Cell Biochem. 2011;112(8):2149-59.

18. Sato S, Suzuki H, Tsujimoto H, Shirato K, Tachiyashiki K, Imaizumi K. Casted-immobilization downregulates glucocorticoid receptor expression in rat slow-twitch soleus muscle. Life Sci. 2011;89(25-26):962-7.

19. Burkholder TJ, Fingado B, Baron S, Lieber RL. Relationship between muscle fiber types and sizes and muscle architectural properties in the mouse hindlimb. J Morphol. 1994;221(2):17790.

20. de Theije CC, Langen RC, Lamers WH, Schols AM, Koehler SE. Distinct responses of protein turnover-regulatory pathways in hypoxia- and semi-starvation-induced muscle atrophy. Am J Physiol Lung Cell Mol Physiol. 2013.

21. Bredman JJ, Wessels A, Weijs WA, Korfage JA, Soffers CA, Moorman AF. Demonstration of 'cardiac-specific' myosin heavy chain in masticatory muscles of human and rabbit. Histochem J. 1991;23(4):160-70.

22. Kon M, Ohiwa N, Honda A, Matsubayashi T, Ikeda T, Akimoto T, et al. Effects of systemic hypoxia on human muscular adaptations to resistance exercise training. Physiol Rep. 2014;2(6).

23. Krock BL, Skuli N, Simon MC. Hypoxia-induced angiogenesis: good and evil. Genes Cancer. 2011;2(12):1117-33.

24. Vilar J, Waeckel L, Bonnin P, Cochain C, Loinard C, Duriez M, et al. Chronic hypoxia-induced angiogenesis normalizes blood pressure in spontaneously hypertensive rats. Circulation research. 2008;103(7):761-9.

25. Weckbach LT, Groesser L, Borgolte J, Pagel JI, Pogoda F, Schymeinsky J, et al. Midkine acts as proangiogenic cytokine in hypoxia-induced angiogenesis. American journal of physiology Heart and circulatory physiology. 2012;303(4):H429-38.

26. Itoh K, Moritani T, Ishida K, Hirofuji C, Taguchi S, Itoh M. Hypoxia-induced fibre type transformation in rat hindlimb muscles. Histochemical and electro-mechanical changes. Eur J Appl Physiol Occup Physiol. 1990;60(5):331-6.

27. Gosker HR, Zeegers MP, Wouters EF, Schols AM. Muscle fibre type shifting in the vastus lateralis of patients with COPD is associated with disease severity: a systematic review and meta-analysis. Thorax. 2007;62(11):944-9.

28. van den Borst B, Schols AM, de Theije C, Boots AW, Kohler SE, Goossens GH, et al. Characterization of the inflammatory and metabolic profile of adipose tissue in a mouse model of chronic hypoxia. J Appl Physiol (1985). 2013;114(11):1619-28.

29. Watson ML, Baehr LM, Reichardt HM, Tuckermann JP, Bodine SC, Furlow JD. A cell-autonomous role for the glucocorticoid receptor in skeletal muscle atrophy induced by systemic glucocorticoid exposure. Am J Physiol Endocrinol Metab. 2012;302(10):E1210-20. 
30. Schakman O, Kalista S, Barbe C, Loumaye A, Thissen JP. Glucocorticoid-induced skeletal muscle atrophy. The international journal of biochemistry \& cell biology. 2013;45(10):2163-72.

31. Roy RR, Gardiner PF, Simpson DR, Edgerton VR. Glucocorticoid-induced atrophy in different fibre types of selected rat jaw and hind-limb muscles. Arch Oral Biol. 1983;28(7):639-43.

32. Stavreva DA, Varticovski L, Hager GL. Complex dynamics of transcription regulation. Biochim Biophys Acta. 2012;1819(7):657-66.

33. Leonard MO, Godson C, Brady HR, Taylor CT. Potentiation of glucocorticoid activity in hypoxia through induction of the glucocorticoid receptor. Journal of immunology. 2005;174(4):2250-7.

34. Livingstone I, Johnson MA, Mastaglia FL. Effects of dexamethasone on fibre subtypes in rat muscle. Neuropathol Appl Neurobiol. 1981;7(5):381-98.

35. Gray S, Wang B, Orihuela Y, Hong EG, Fisch S, Haldar S, et al. Regulation of gluconeogenesis by Kruppel-like factor 15. Cell metabolism. 2007;5(4):305-12.

36. Hickson RC, Wegrzyn LE, Osborne DF, Karl IE. Glutamine interferes with glucocorticoid-induced expression of glutamine synthetase in skeletal muscle. The American journal of physiology. 1996;270(5 Pt 1):E912-7.

37. Shimizu N, Yoshikawa N, Ito N, Maruyama T, Suzuki Y, Takeda S-i, et al. Crosstalk between glucocorticoid receptor and nutritional sensor mTOR in skeletal muscle. Cell metabolism. 2011;13:170-82.

38. Park CW, Ryu KY. Cellular ubiquitin pool dynamics and homeostasis. BMB Rep. 2014;47(9):47582.

39. Koncarevic A, Jackman RW, Kandarian SC. The ubiquitin-protein ligase Nedd4 targets Notch1 in skeletal muscle and distinguishes the subset of atrophies caused by reduced muscle tension. FASEB journal : official publication of the Federation of American Societies for Experimental Biology. 2007;21(2):427-37.

40. Regula KM, Ens K, Kirshenbaum LA. Inducible expression of BNIP3 provokes mitochondrial defects and hypoxia-mediated cell death of ventricular myocytes. Circulation research. 2002;91(3):226-31.

41. Bellot G, Garcia-Medina R, Gounon P, Chiche J, Roux D, Pouysségur J, et al. Hypoxia-induced autophagy is mediated through hypoxia-inducible factor induction of BNIP3 and BNIP3L via their BH3 domains. Mol Cell Biol. 2009;29:2570-81.

42. Zhang H, Bosch-Marce M, Shimoda LA, Tan YS, Baek JH, Wesley JB, et al. Mitochondrial autophagy is an HIF-1-dependent adaptive metabolic response to hypoxia. J Biol Chem. 2008;283(16):10892-903.

43. Klionsky DJ, Abdalla FC, Abeliovich H, Abraham RT, Acevedo-Arozena A, Adeli K, et al. Guidelines for the use and interpretation of assays for monitoring autophagy. Autophagy. 2012;8(4):445-544.

44. Lunde IG, Anton SL, Bruusgaard JC, Rana ZA, Ellefsen S, Gundersen K. Hypoxia inducible factor 1 links fast-patterned muscle activity and fast muscle phenotype in rats. The Journal of physiology. 2011;589(Pt 6):1443-54. 
45. Gamboa JL, Garcia-Cazarin ML, Andrade FH. Chronic hypoxia increases insulin-stimulated glucose uptake in mouse soleus muscle. Am J Physiol Regul integr comp physiol. 2011;300:R8591.

46. Breen E, Tang K, Olfert M, Knapp A, Wagner P. Skeletal muscle capillarity during hypoxia: VEGF and its activation. High altitude medicine \& biology. 2008;9(2):158-66

47. VEGF-A gene expression profile on BioGPS website. Available from: http://biogps. org/\#goto=genereport\&id=7422.

48. Nauck M, Karakiulakis G, Perruchoud AP, Papakonstantinou E, Roth M. Corticosteroids inhibit the expression of the vascular endothelial growth factor gene in human vascular smooth muscle cells. European journal of pharmacology. 1998;341(2-3):309-15.

49. Murakami S, Fujino H, Takeda I, Momota R, Kumagishi K, Ohtsuka A. Comparison of capillary architecture between slow and fast muscles in rats using a confocal laser scanning microscope. Acta medica Okayama. 2010;64:11-8.

50. Deveci D, Marshall JM, Egginton S. Relationship between capillary angiogenesis, fiber type, and fiber size in chronic systemic hypoxia. American journal of physiology Heart and circulatory physiology. 2001;281:H241-52.

51. Wust RC, Jaspers RT, van Heijst AF, Hopman MT, Hoofd LJ, van der Laarse WJ, et al. Regionspecific adaptations in determinants of rat skeletal muscle oxygenation to chronic hypoxia. American journal of physiology Heart and circulatory physiology. 2009;297(1):H364-74.

52. Pereira ER, Frudd K, Awad W, Hendershot LM. Endoplasmic reticulum (ER) stress and hypoxia response pathways interact to potentiate hypoxia-inducible factor 1 (HIF-1) transcriptional activity on targets like vascular endothelial growth factor (VEGF). J Biol Chem. 2014;289(6):335264.

53. Bishop B, Silva G, Krasney J, Nakano H, Roberts A, Farkas G, et al. Ambient temperature modulates hypoxic-induced changes in rat body temperature and activity differentially. American journal of physiology Regulatory, integrative and comparative physiology. 2001;280(4):R11906.

54. Glass DJ. Skeletal muscle hypertrophy and atrophy signaling pathways. The international journal of biochemistry \& cell biology. 2005;37(10):1974-84.

55. Gomes MD, Lecker SH, Jagoe RT, Navon A, Goldberg AL. Atrogin-1, a muscle-specific F-box protein highly expressed during muscle atrophy. Proc Natl Acad Sci U S A. 2001;98(25):144405.

56. Sandri M. Autophagy in skeletal muscle. FEBS letters. 2010;584(7):1411-6.

57. Mizushima N, Ohsumi Y, Yoshimori T. Autophagosome formation in mammalian cells. Cell Struct Funct. 2002;27(6):421-9.

58. Max SR, Thomas JW, Banner C, Vitkovic L, Konagaya M, Konagaya Y. Glucocorticoid receptormediated induction of glutamine synthetase in skeletal muscle cells in vitro. Endocrinology. 1987;120(3):1179-83.

59. Ma K, Mallidis C, Bhasin S, Mahabadi V, Artaza J, Gonzalez-Cadavid N, et al. Glucocorticoidinduced skeletal muscle atrophy is associated with upregulation of myostatin gene expression. Am J Physiol Endocrinol Metab. 2003;285(2):E363-71. 
Hypoxia-induced muscle atrophy and impaired regulation of protein turnover are partially dependent on muscle GR signaling

C.C. de Theije, A.M.W.J. Schols, W.H. Lamers, J.J.M. Ceelen, R.H. van Gorp, J.J.R. Hermans, S.E. Köhler, R.C.J. Langen

Submitted 


\section{Abstract:}

Hypoxia may contribute to muscle wasting in COPD. Hypoxemia induces skeletal muscle atrophy in mice, which in part can be attributed to a reduction of food intake. As increased glucocorticoid concentrations and glucocorticoid receptor (GR) signaling have been implicated in fasting-induced muscle atrophy, we hypothesized that hypoxia-induced muscle atrophy is GR dependent. Muscle-specific GR knockout (mGRKO) and control mice were subjected to normoxia, normobaric hypoxia ( $8 \%$ oxygen) or pair-fed to the hypoxia group for 4 days. Hypoxia and reduced food-intake resulted in elevated corticosterone plasma concentrations and increased expression of the GR-dependent genes KIf15, Glul and Foxo1 in the gastrocnemius muscle. GR deficiency prevented muscle atrophy in the pair-fed group but not in the hypoxic group. GR deficiency blunted the elevated expression of autophagy-lysosomal degradation (Map1/c3B, Bnip3) related genes in both hypoxic and pair-fed mice, but did not affect overall autophagy measured by MAP1LC3B-I and -II. Hypoxia-induced expression of the Ub 26S-proteasome E3 ligases (Murf1, Atrogin-1) was suppressed in the pair-fed but not in the hypoxic group. Reduction of food intake to levels consumed by hypoxic animals, resulted in reduced 4E-BP1-, p70S6K1-, and RPS6 phosphorylation under normoxia, while this suppression of mTORC1 activity was lacking under hypoxic conditions. This lack of mTORC1 inhibition under hypoxia required GR signaling and did not involve the established AKT/TSC2 or AKT/mTOR axis nor alterations of Redd1 or KIf15 expression. We conclude that hypoxia-induced muscle atrophy is partially dependent on muscle GR signaling. Furthermore, mTORC1 suppression by reduced anabolic stimuli is partially reversed by GR under hypoxic conditions. 


\section{Introduction}

Chronic obstructive pulmonary disease (COPD) is a global leading cause of morbidity and disability [1]. Weight loss and muscle atrophy are common features in advanced COPD. Muscle atrophy is a strong predictor of mortality and significantly increases disease burden [1-5]. In addition to nutritional imbalance, glucocorticoid use, systemic inflammation and progressive inactivity, hypoxemia has been implicated as trigger for muscle atrophy in COPD patients [6]. Previously, it was shown that hypoxia causes muscle atrophy in rat models [7, 8]. Our group also confirmed these findings in a normobaric hypoxia mouse model [9], and revealed that muscle atrophy was attributable to hypoxia-induced reduction of food intake, and hypoxia-specific effects. The mechanisms of fasting-induced muscle atrophy involve elevated glucocorticoid concentrations and glucocorticoid receptor (GR) signaling [10], which affect protein synthesis and proteolysis in skeletal muscle. Adrenalectomized rats display reduced muscle protein breakdown, which is restored by glucocorticoid administration [11]. Moreover, in muscle-specific GR knock-out (mGRKO) mice, fasting-induced reductions in fiber cross sectional area (FCSA) were absent, which was accompanied by attenuated activation of the proteolytic machinery [12]. GR affects muscle protein turnover by genomic and non-genomic actions [13]. Genomic effects include activation of protein degradation by the ubiquitin proteasomal system (UPS) mediated by GR transactivation. Forkhead box protein $\mathrm{O} 1$ (Foxo1) is upregulated in a GR-specific manner, and contributes to the subsequent induction of expression of atrogenes, such as tripartite motif containing 63 (Trim63/Murf1) and F-box only protein 32 (Fbxo32/Mafbx/Atrogin-1) [14-16]. GR also up regulates Krüppel-like factor 15 (KIf15) expression. In addition to its involvement in the control of proteolysis via regulation of Murf1 and Atrogin-1, Klf15 also contributes to the regulation of protein synthesis [17]. Glutamate-ammonia ligase (GLUL) is an enzyme involved in muscle amino acid metabolism during fasting [18], and its expression is also stimulated by GC through GR-responsive elements (GRE) in the Glul promoter [19]. Furthermore, transcriptional control of DNA-damage-inducible transcript 4 protein (Ddit4/Redd1) by GR results in suppressed protein synthesis and activation of autophagy through inhibition of mTOR [20]. Non-genomic GR actions involve suppression of protein synthesis through the inhibition of the phosphatidylinositol-4,5-bisphosphate 3-kinase (PI3K) / protein kinase B (PKB/AKT) / TSC1/2 / mammalian target of rapamycin (mTOR) signaling pathway, in which GR activation prevents association of PI3K with insulin receptor substrate-1 (IRS1) [12]. As we previously reported [21], hypoxia-exposed mice show alterations in proteolysis- and protein synthesis signaling in skeletal muscle. We therefore hypothesized that muscle GR signaling in response to elevated circulating corticosterone concentrations is required for elevated protein degradation and also af- 
fects the regulation of protein synthesis during hypoxia-induced muscle atrophy.

\section{Methods}

\section{Animals and tissue collection.}

All mouse studies were carried out according to a protocol approved by the Institutional Animal Care Committee of Maastricht University. 12-week-old male muscle-specific GR knockout mice (mGRKO) were generated by crossing the transgenic Mlc1f-Cre+/- mice (Cre recombinase under control of the fast myosin light chain 1 promoter) [22] with mice bearing floxed $\mathrm{Gr}$ alleles (Grfl/fl) [23] on a C57BL/6 background, resulting in the ablation of GR in MyHC type II (fast) muscle cells. Twenty-four control (littermates) and 24 mGRKO mice were randomly divided into 3 groups: normoxia (N), normoxia pair-fed to hypoxic animals (PF), and normobaric hypoxia $(\mathrm{H})$. All mice were housed in experimental chambers at $21^{\circ} \mathrm{C}$ with a 12-hour dark/light cycle. Mice received standard chow (V1534-000 Ssniff R/M-H, Ssniff Spezialdiäten) and water ad libitum. After 5 days of acclimatization, normoxic and pair-fed groups were exposed to $21 \% \mathrm{O} 2$ and the hypoxic group to normobaric hypoxia. Using the proOX system P110 (BioSpherix), oxygen levels were reduced in a stepwise manner to $12 \%$ (day 0 ), $10 \%$ (day 1 ), and finally $8 \%(61 \mathrm{mmHg})$ on day 2 , which was then maintained for the remainder of the experiment. To assess the effects of reduced food intake during hypoxia, pair-fed animals received daily the amount of food consumed by the hypoxic mice. Tissue and plasma were isolated at day 4 and frozen immediately in liquid nitrogen for further analysis. Tissue weights were corrected for body weight at the start of the experiment.

\section{Corticosterone measurement.}

Plasma corticosterone concentrations were determined by HPLC. A carboxylic

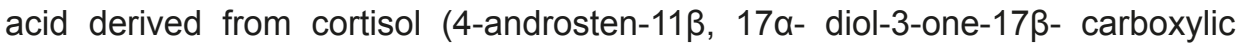
acid), was synthetized and purified as described previously [24] and was added as internal control to the plasma and corticosterone standards. Samples and standards were acidified with phosphoric acid, and steroid hormones were subsequently extracted from the plasma with diethyl ether, and dried with nitrogen gas. The residues were dissolved in acetone and incubated with sulphuric acid for 20 minutes to convert the steroids into fluorescent products [25]. Following addition of 100 volumes of diethylether and 5 volumes of water, the fluorescent products were extracted from the aqueous sulfuric acid phase. After evaporating the organic phase under a stream of nitrogen, the residues were dissolved to be analyzed by HPLC as described [24]. 


\section{In vivo electroporation of gastrocnemius muscle.}

Plasmids were prepared using an endotoxin-free maxi prep kit (Qiagen) and dissolved in $0.9 \%$ saline. Plasmid mix consisted of $1: 1$ glucocorticoid receptor response element (GRE)-luciferase construct [26] and pVAX-lacZ plasmid (Invitrogen) encoding $\beta$-galactosidase. Mice were anesthetized with isoflurane and injected subcutaneously with buprenorphine (Temgesic) as analgesic. Skin covering the gastrocnemius muscle was shaved and disinfected. Using a bent 0.3 $\mathrm{ml}$ syringe with a 30 gauge needle (Becton Dickinson), 5 times $10 \mu$ plasmid (50 $\mu \mathrm{g}$ total) were injected at different sites into the gastrocnemius muscle. Custombuilt mouse electrodes with electrolyte crème (Parker Laboratories) were placed on both sides of the gastrocnemius muscle on top of the skin. 8 Pulses of 150 Volt/cm, with $20 \mathrm{~ms}$ pulse length and a 1 second interval were applied using the BTX square wave electroporator (BTX Harvard apparatus). The direction of the pulse was inverted after 4 pulses to increase efficiency. After 21 days of recovery, mice were exposed to experimental conditions. Muscles were snap frozen in liquid nitrogen, and ground to powder using an N2-cooled steel mortar. Muscle powder was lysed in passive lysis buffer (Promega) and assessed for luciferase (Promega) and $\beta$-gal (Tropix) activity.

\section{RT-qPCR.}

Total RNA was isolated using TRI REAGENT ${ }^{\text {TM }}$ (Sigma-Aldrich) and further purified by precipitation with $2 \mathrm{M} \mathrm{LiCl}$. cDNA synthesis was performed with random hexamer primers on denatured RNA using the Transcriptor First Strand cDNA Synthesis Kit (Roche). Real-time quantitative PCR was performed in the iQ5 thermal cycler (Biorad) using the qPCR SyBr Green Fluorescein Mix (Abgene). qPCR primers were designed using Primer Express 2.0 software (Applied Biosystems) and ordered from Sigma Genosys (Table 1). Expression of genes was normalized to $18 \mathrm{~S}$ ribosomal RNA.

\begin{tabular}{llll}
\hline \hline Gene & NCBI access $\mathbf{n r}$. & Forward primer (5' to 3') & Reverse primer (5'to 3') \\
\hline 18S & NR_003278.1 & AGTTAGCATGCCAGAGTCTCG & TGCATGGCCGTTCTTAGTTG \\
Gr & NM_008173.3 & CGCCAAGTGATTGCCGC & TGTAGAAGGGTCATTTGGTCATCCA \\
Glul & NM_008131.3 & GGCCATGCGGGAGGAGA & GGTGCCTCTTGCTCAGTTTGTCA \\
Fox01 & NM_019739.3 & AAGAGCGTGCCCTACTTCAAG & CCATGGACGCAGCTCTTCTC \\
KIf15 & NM_023184.3 & TGCAGCAAGATGTACACCAAGAG & ATCGCCGGTGCCTTGAC \\
Redd1 & NM_029083.2 & CGGGCCGGAGGAAGACT & CTGCATCAGGTTGGCACACA \\
Murf1 & NM_00103904 & TGTCTGGAGGTCGTTTCCG & CTCGTCTTCGTGTTCCTTGC \\
Atrogin-1 & NM_026346.2 & ACCGGCTACTGTGGAAGAGA & CCTTCCAGGAGAGAATGTGG \\
Map1/c3B & NM_026160.4 & GAGCAGCACCCCACCAAGAT & CGTGGTCAGGCACCAGGAA \\
Bnip3 & NM_009760.4 & CCATGTCGCAGAGCGGG & GACGGAGGCTGGAACGC \\
\hline
\end{tabular}

Table 1. Sequences of primers used for RT-qPCR to asses expression of the indicated genes. 


\section{Western blot.}

Western blot was performed according to procedures previously described [21]. In short, gastrocnemius muscle was ground to powder and lysed. Total protein concentration was determined and $12.5 \mu \mathrm{g} / \mathrm{lane}$ was used for Western blotting. PonceauS staining was used to control and correct for protein loading. The membrane was incubated overnight at $4^{\circ} \mathrm{C}$ with primary antibodies; GR: \#12041, S6: \#2271, p-S6(Ser235/236): \#4856, 4E-BP1: \#9452, p-4E-BP1(Ser65): \#9451, p-4E-BP1(Thr37/46): \#9459, mTOR: \#2983, p-mTOR(Ser2448): \#2971, pmTOR(Ser2481): \#2974, AKT: \#9272, p-AKT(Ser473): \#9271, TSC2: \#4308, p-TSC2(Ser939): \#3615, p-TSC2(Thr1462): \#3617 (Cell Signaling Technology). Blots were probed with a horseradish peroxidase-conjugated secondary antibody and visualized by chemiluminescence in a LAS-3000 Luminescent Image analyzer (Fujifilm). Bands were quantified using the AIDA software (Fujifilm). Figures mainly show the ratios of phosphorylated over total protein of interest, total levels of the protein of interest is shown when it affects the ratio's.

\section{FCSA determination.}

Gastrocnemius muscle was embedded in Tissue-Tek (Sakura Finetek) and sectioned on a Leica CM3050 S cryostat at $-20^{\circ} \mathrm{C}$. Subsequently, serial cross-sections $(5 \mu \mathrm{m})$ were stained with anti-laminin (\#L-9393) (Sigma-Aldrich) to determine the fiber cross-sectional area (FCSA). The sections were incubated with the following secondary antibody: Alexa Fluor 350 (\#A-21426) (Invitrogen). Digital images of the stained sections were taken at 200X total magnification using an Eclipse E800 microscope (Nikon) connected to a digital camera (DXM, $1200 \mathrm{NF}$, Nikon). The FCSA, in the glycolytic region of the gastrocnemius, was measured for $>100$ individual fibers per animal, using the Lucia software (version 4.81). A distribution curve was composed of all measured fibers within the groups (>800 fibers).

\section{Statistics.}

Data are shown as means \pm SEM. Comparisons were computed with SPSS version 20 (SPSS Inc.). Statistical significance between groups within a genotype (WT or mGRKO), was tested using a one-way ANOVA with a post-hoc test. The type of post-hoc analysis was chosen on the basis of the data variance (Levene's test), with the Tukey test for data with equal variance, and the Games-Howell test for all other data. Statistical significance between genotypes per group was assessed using the independent-samples T-test. P-values $<0.05$ were considered to be statistically significant and $0.05 \leq \mathrm{P} \leq 0.1$ as indicating a trend. 


\section{Results}

Hypoxia increases corticosterone plasma concentrations and induces muscle glucocorticoid receptor signaling.

Four days of hypoxia resulted in elevated corticosterone concentrations (Fig. 1A). This increase was in part attributable to the reduced food intake (PF: 2.0-fold, H: 3.0-fold). To address whether the elevated circulating corticosterone concentrations activated muscle glucocorticoid receptor (GR) signaling in vivo, a GREreporter (luciferase) plasmid was electroporated into the gastrocnemius muscle prior to 4 days exposure to $\mathrm{N}, \mathrm{H}$ or PF conditions (Fig. 1B). The transcriptional activity of GR was increased under hypoxic conditions (1.9-fold) and even more so in the PF group (3.1-fold).

A

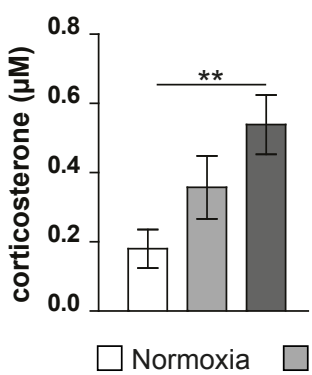

B

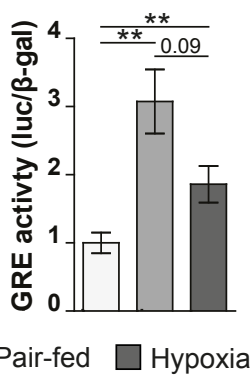

Figure 1. Hypoxia raises corticosterone plasma concentrations and induces muscle glucocorticoid receptor signaling. (A): plasma corticosterone concentration at day 4 (B): GR transcriptional activity in gastrocnemius muscle at day 4. GRE-reporter plasmid (luciferase) data were corrected for control ( $\beta$-galactosidase) plasmid data. Significant differences between groups at a given time point are indicated by asterisks, with ${ }^{*}=P<0.05,{ }^{* *}=P \leq 0.01$ or ${ }^{* * *}=P \leq 0.001$. Trends $(0.05 \leq P \leq 0.1)$ are indicated by the specific $P$-value. ( $n=8$ per group).

GR target genes are differentially expressed in mGRKO.

To assess the importance of GR-signaling during hypoxia-induced muscle atrophy, muscle-specific GR knockout mice were employed. In these mice, GR protein (Fig. 2A) and Gr mRNA (Fig. 2B) were reduced by $60 \%$ in the mGRKO gastrocnemius muscles. Interestingly, both in control and mGRKO mice, $\mathrm{Gr}$ mRNA concentration was increased by hypoxia, but not by reduced food-intake alone (Fig. 2B). Subsequently, mRNA expression of postulated GR-target genes related to muscle protein turnover was evaluated. Basal Glul transcript levels were lower (0.8 fold) in mGRKO compared to control mice (Fig. 2C). Both Glul (Fig. 2C) and Foxo1 (Fig. 2D) expression was increased in response to hypoxia in muscle of control, but not of mGRKO mice. Hypoxia and a reduction of food intake increased Klf15 expression to a similar extent in control mice (Fig. 2E). In contrast, in mGRKO mice expression of KIf15 was not changed under hypoxic conditions but was increased in PF mice. Redd1 expression increased in 
response to reduced food intake but not hypoxia in control and mGRKO mice (Fig. 2F). Baseline plasma corticosterone concentrations were comparable in both genotypes and showed similar increases in response to a reduced food intake or hypoxia (Fig. 2G). To conclude, corticosterone concentrations increased equally in control and mGRKO mice in response to hypoxia. Hypoxia-induced expression of Glul and Foxo1 in skeletal muscle was attributable to the reduced food intake and GR dependent.
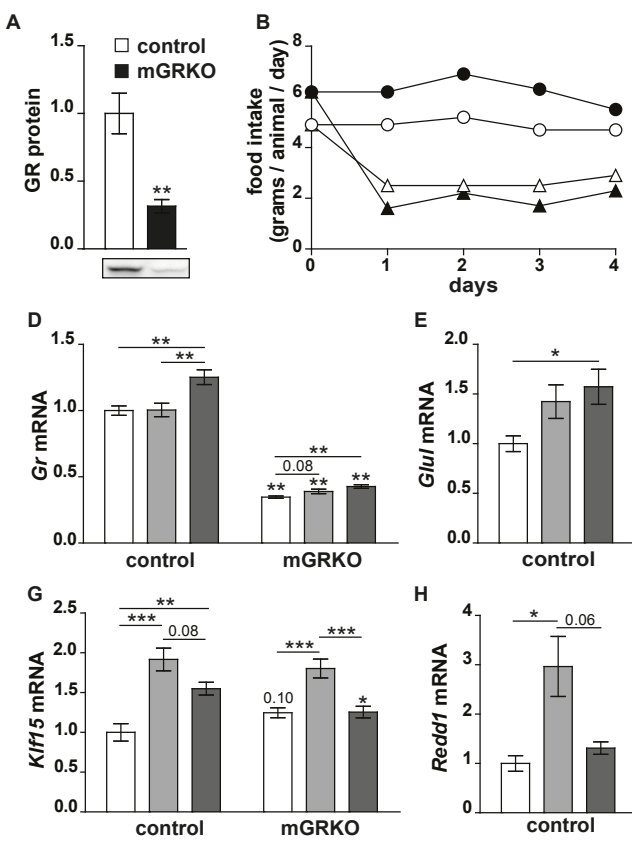

E

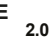

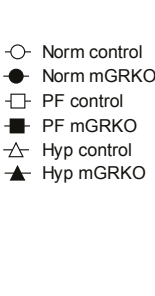

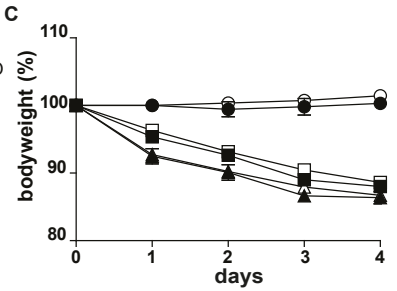

F
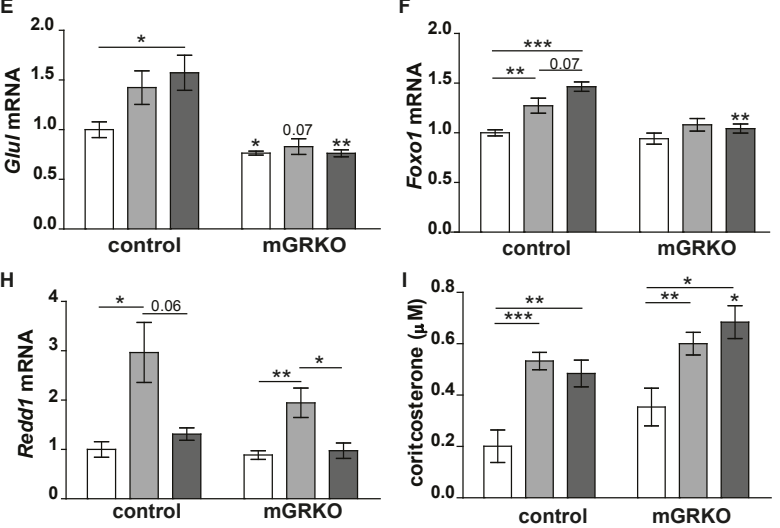

$\square$ Normoxia $\square$ Pair-fed $\square$ Hypoxia

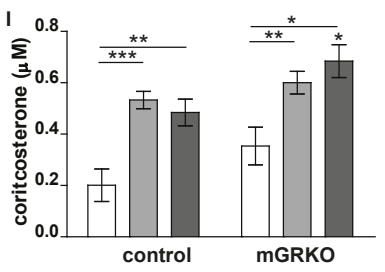

Figure 2. GR target genes are differentially expressed in mGRKO in response to reduced foodintake and hypoxia. (A) Protein levels of GR in the gastrocnemius muscle odf both genotypes. (B): Average food-intake per animal per day (no statistics). (C): changes in bodyweight as a percentage of start weight (no statistics). To asses changes in the GR signaling pathway, mRNA expression of $\mathrm{Gr}$ and transcriptional targets of GR were measured in the gastrocnemius muscle of (D): $G r(E)$ : Glul, (F): Foxo1, (G): Klf15, (H) Redd1. (I): Plasma corticosterone concentration. All mRNA concentrations were corrected by $18 \mathrm{~S}$ concentration. Significant differences between groups within control or mGRKO mice are indicated by asterisks on horizontal lines. Significant differences between control and mGRKO mice per condition are indicated by asterisks or trend above the column. ${ }^{*}=P<0.05$, ${ }^{* *}=P \leq 0.01$ or ${ }^{* * *}=P \leq 0.001$. Trends $(0.05 \leq P \leq 0.1)$ are indicated by the specific $P$-value $(n=6-8)$.

Hypoxia-induced muscle atrophy is not prevented by muscle GR deficiency. Gastrocnemius muscle weights of mGRKO animals appeared slightly increased $(4 \%, p=0.06)$ compared to control mice under basal conditions (Fig. 3A). In control animals, the mass of the gastrocnemius muscle decreased under hypoxia, which was in part attributable to reduced food intake (PF: $-7.1 \%, \mathrm{H}:-12.9 \%$ ). In contrast, in mGRKO mice, muscle mass was preserved under pair-fed condi- 
tions. Hypoxia-induced muscle atrophy was still observed in mGRKO mice, and muscle loss was not significantly different from control ( $\mathrm{H}$ vs $\mathrm{N}$; Control: $-12.9 \%$ vs mGRKO: $-8.5 \%$ ). In control mice, FCSA are reduced in response to paired feeding and hypoxia (N vs PF vs H, 2184 vs 1789 vs 1868 um2) (Fig. 3B-E). In line with muscle weights, hypoxia resulted in a reduced FCSA in the mGRKO mice ( $\mathrm{N}$ vs H, 2027 vs 1607 um2, p=0.07). Furthermore, FCSA was not changed by reduced food intake in the PF-group. Interestingly, the pattern of fiber size redistribution following hypoxia differed between WT and mGRKO muscle (Fig. 3D,E), with a mainly large-to-median shift in FCSA in WT muscle vs a large-to-small shift in $\mathrm{mGRKO}$ muscle. Combined, these data revealed that muscle atrophy attributable to the hypoxia-induced reduction in food intake was GR-dependent, while the hypoxia-specific reduction of muscle mass was not.

A

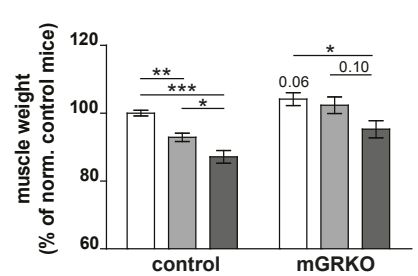

C

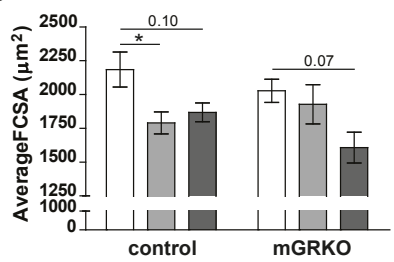

B

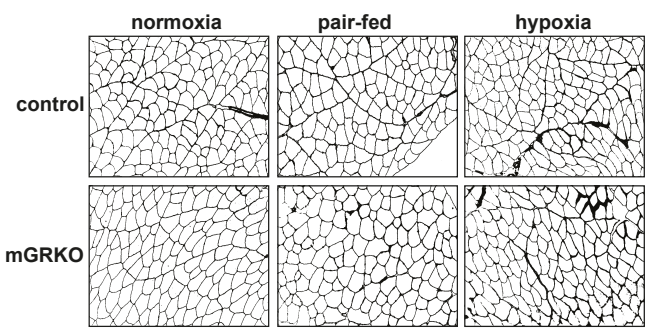

D

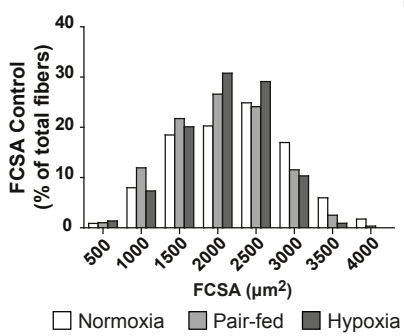

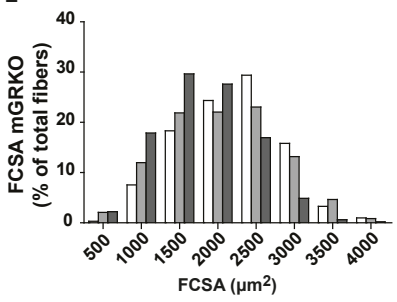

Figure 3. Hypoxia-induced muscle atrophy is not prevented by muscle GR deficiency. (A): Gastrocnemius muscle weight after 4 days of hypoxia or pair-fed conditions in control and mGRKO mice as percentage of the muscle mass of normoxic control mice. (B): representative images of laminin staining. (C): Average FCSA of glycolytic region in gastrocnemius muscle of control and mGRKO mice. (D): FCSA distribution of the glycolytic region in gastrocnemius muscle of control mice (no significance indicated). (E): FCSA distribution of the glycolytic region in gastrocnemius muscle of mGRKO mice (no significance indicated). Significant differences between groups at a given time point are indicated by asterisks, with ${ }^{*}=P<0.05,{ }^{* *}=P \leq 0.01$ or ${ }^{* * *}=P \leq 0.001$. Trends $(0.05 \leq P \leq 0.1)$ are indicated by the specific P-value ( $n=6-8)$.

GR deficiency differentially suppresses ALP- and UPS proteolytic signaling.

Muscle atrophy may involve elevated protein degradation through the ubiquitin 26S-proteasome system (UPS) and the autophagy lysosomal pathway (ALP). Expression of E3-ubiquitin ( $\mathrm{Ub}$ ) ligases Murf1 and Atrogin-1 was investigated to 
assess UPS activation [27]. Basal Murf1 and Atrogin-1 expression was similar in control and mGRKO mice (Fig. 4A, B). In control mice, reduced food intake and hypoxia equally elevated the expression of both Ub ligases, whereas in the mGRKO mice the response to reduced food intake was diminished. Basal expression levels of the ALP-related genes Map1/c3B and Bnip3 were similar in control and mGRKO animals (Fig. 4C, D). Reduced food intake and hypoxia increased expression of both ALP related genes to a similar extent in both controls and $\mathrm{mGRKO}$ mice, although the response was blunted in the latter. Reduced food intake and hypoxia both reduced MAP1LC3B-I protein levels in control and mGRKO mice (Fig. 4E), despite elevated mRNA levels. However, MAP1LC3B-II was not increased as expected but slightly reduced under hypoxic conditions and normal in response to reduced food intake (Fig. 4F). To summarize, whereas reduced food intake under normoxia induced mRNA expression of Murf1 and Atrogin-1 GR-dependently, the increased transcript levels observed following hypoxia of these E3-ubiquitin ligases involved in UPS-mediated proteolysis were minimally affected by GR deficiency. Furthermore, GR deficiency similarly blunted ALPrelated gene expression in response to hypoxia and reduced food intake, but MAP1LC3B-I and -II protein levels do not suggest differences in the ALP activity.
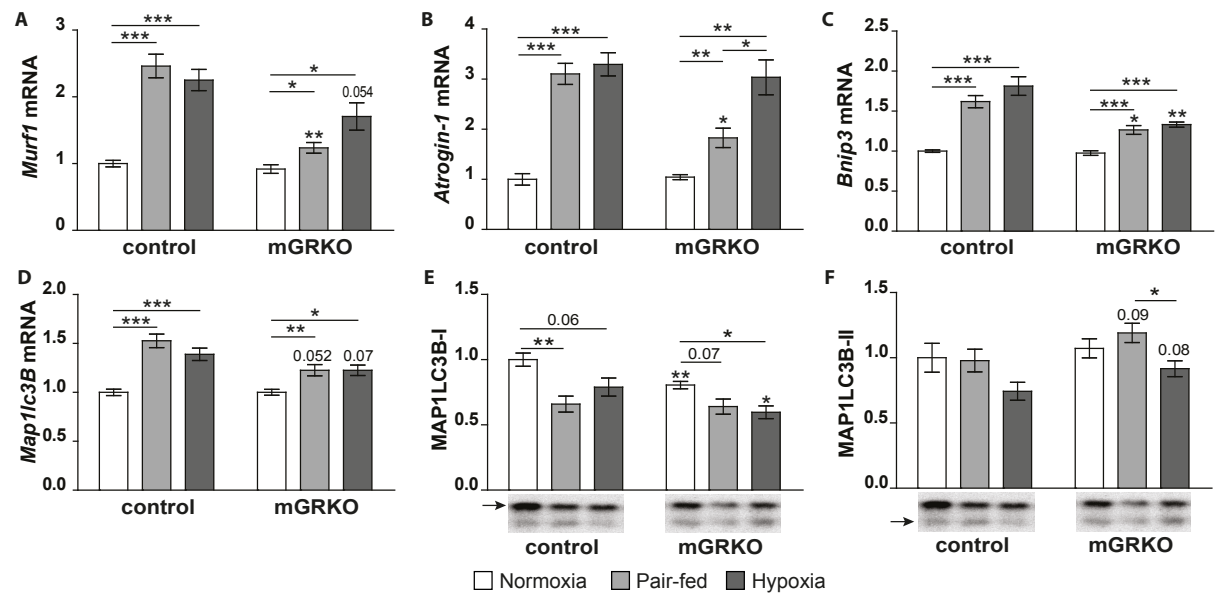

Figure 4. GR deficiency differentially suppresses ALP- and UPS proteolytic signaling. (A): Murf1 expression. (B): Atrogin-1 expression. (C): Bnip3 expression. (D): Map1/c3B expression. (E): MAP1LC3B-I protein. (F): MAP1LC3B-II protein. All mRNA concentrations were corrected by 18S concentration. Significant differences between groups within control or $\mathrm{mGRKO}$ mice are indicated by asterisks on horizontal lines. Significant differences between control and mGRKO mice per condition are indicated by asterisks or trend above the column. ${ }^{*}=\mathrm{P}<0.05,{ }^{* *}=\mathrm{P} \leq 0.01$ or ${ }^{* *}=\mathrm{P} \leq 0.001$. Trends $(0.05 \leq P \leq 0.1)$ are indicated by the specific $P$-value $(n=6-8)$.

\section{Altered regulation of protein synthesis signaling by hypoxia is GR depen- dent.}

The mTORC1 pathway is central in the regulation of protein synthesis signal- 
ing [28, 29]. Ribosomal protein S6 kinase, 70kDa, polypeptide 1 (P70S6K1) is directly phosphorylated by mTOR. P70S6K1 phosphorylates ribosomal protein S6 (RPS6) by which it controls protein synthesis. P70S6K1 and RPS6 concentrations were unaffected by both experimental conditions and mouse model. As expected, reduced food intake lowered the P70S6K1 and RPS6 phosphorylation ratio suggesting suppressed protein synthesis (Fig. 5A-C). In contrast, the food-dependent reduction of the P70S6K1 and RPS6 phosphorylation ratios was absent under hypoxic conditions. GR deficiency reduced baseline P70S6K1 and RPS6 phosphorylation ratios of normoxic controls. P70S6K1 and RPS6 phosphorylation ratios were further lowered by reduced food intake in the mGRKO, and importantly, in contrast to control mice, P70S6K1 and RPS6 phosphorylation ratios were similarly suppressed by hypoxia. Alterations in the 4E-BP1 Ser65 phosphorylation ratio mirrored the changes observed for P70S6K1 and RPS6 phosphorylation (Fig. 5C, D). 4E-BP1 threonine (Thr) -37 and -46 phosphorylation ratios were hardly affected by the conditions and mouse model (Fig. 5C, E). $4 \mathrm{E}-\mathrm{BP} 1$ protein concentrations were equally increased by hypoxia and reduced food intake in control but not in GR-deficient mice (Fig. 5C, F). Combined, these data indicate that inhibition of $\mathrm{mTORC} 1$ by reduced food-intake does not require muscle GR signaling, whereas the absence of mTORC1 inhibition under hypoxic conditions is GR-dependent.
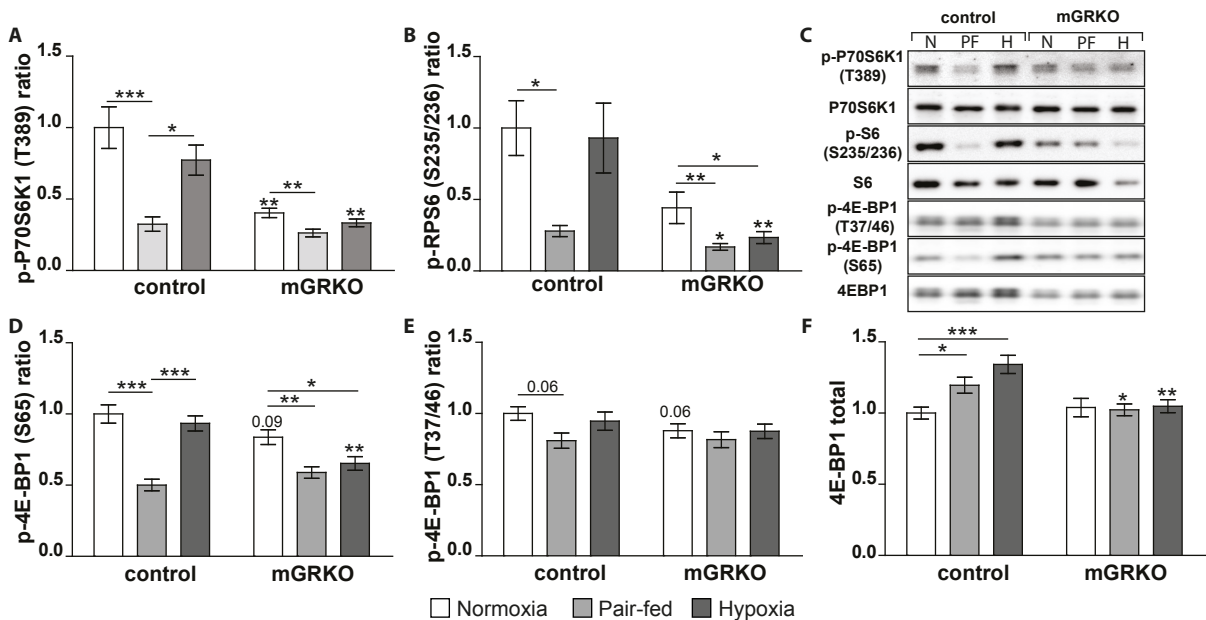

Figure 5. Altered regulation of protein synthesis signaling by hypoxia is GR dependent. (A): phosphorylation ratio of P70S6K1 at Thr389. (B): phosphorylation ratio of RPS6 at Ser235/236. (C): representative images of Western blots. (D): phosphorylation ratio of 4E-BP1 at Ser65. (E): phosphorylation ratio of 4E-BP1 at Thr37/46. (F): total 4E-BP1. Significant differences between groups within control or mGRKO mice are indicated by asterisks on horizontal lines. Significant differences between control and mGRKO mice per condition are indicated by asterisks or trend above the column. *= $\mathrm{P}<0.05,{ }^{* *}=\mathrm{P} \leq 0.01$ or ${ }^{* * *}=\mathrm{P} \leq 0.001$. Trends $(0.05 \leq \mathrm{P} \leq 0.1)$ are indicated by the specific $\mathrm{P}$-value $(n=6-8)$. 
Perturbations in signaling upstream of mTOR are attributable to hypoxiainduced decreases in food intake and mainly independent of GR.

mTOR activity is controlled through direct phosphorylation of Ser2448 by AKT, and AKT-mediated inhibition of TSC2 through phosphorylation of sites Ser939 and Thr1462 in TSC2. AKT protein concentrations were unaffected by experimental conditions or mouse model (Fig. 6G). Basal AKT Ser473 phosphorylation ratio in $\mathrm{mGRKO}$ mice was slightly reduced compared to control $(p=0.06)$ (Fig. 6A, G). In control mice, hypoxia and reduced food intake equally lowered the AKT phosphorylation ratio, whereas in mGRKO mice no further reduction was seen. mTOR protein levels were unaffected by experimental conditions or mouse model (Fig. 6H). GR deficiency significantly lowered the mTOR Ser2448 phosphorylation ratio (Fig.. 6B, H). Reductions in mTOR Ser2448 phosphorylation ratio in response to hypoxia and decreased food intake were similar and did not differ between control and mGRKO mice. mTOR Ser2481 autophosphorylation is postulated to reflect mTOR activity [30]. Except for a decrease in the phosphorylation ratio in response to hypoxia in mGRKO mice, no changes were observed (Fig. 6C, H). TSC2 phosphorylation (Ser939 and Thr1462) ratios were not affected by any condition (Fig. 6D, E, I). Total TSC2 was lowered by reduced food intake in control mice alone (Fig. 6F, I). Combined, these data indicate that the AKT/TSC2 signaling is not involved in suppression of mTOR by reduced food intake, whereas the reduction of the AKT/mTOR signaling by reduced food intake is intact under hypoxic conditions and is independent of GR signaling.
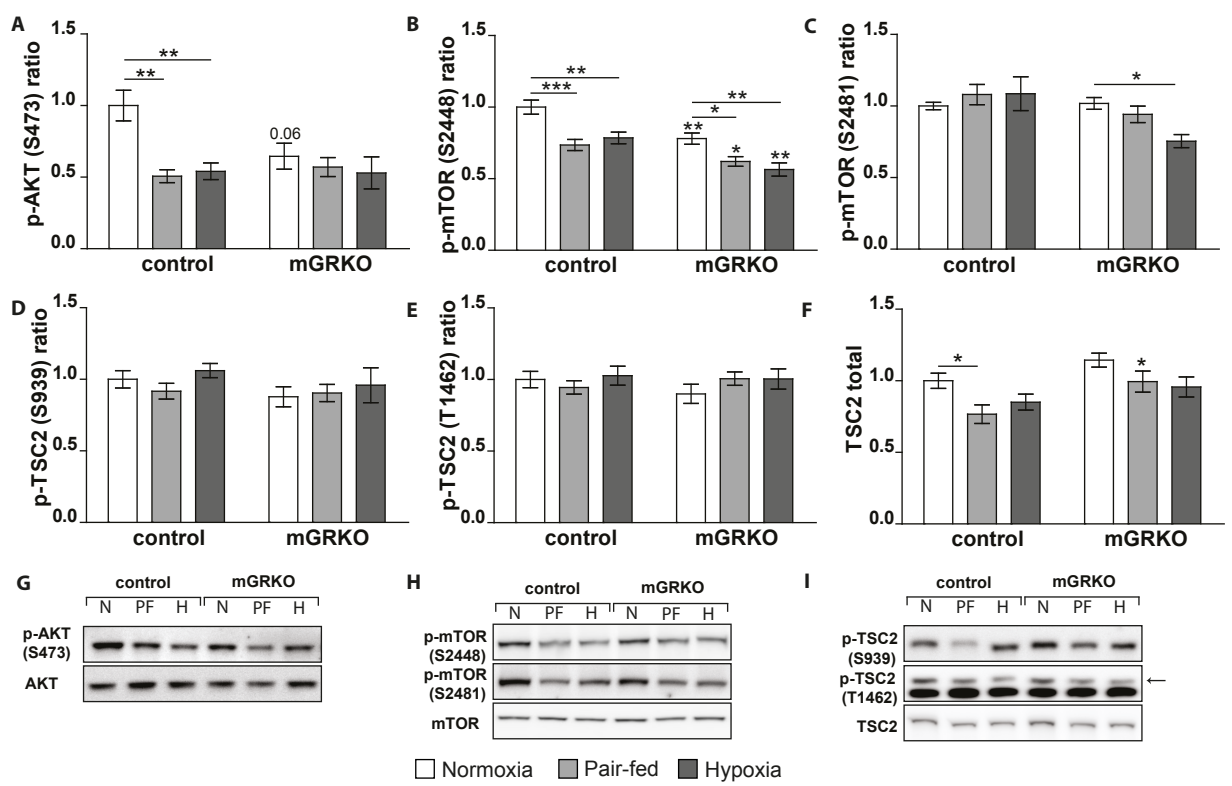
Figure 6. Perturbations in signaling upstream of $m$ TOR are attributable to hypoxia-induced decreases in food intake and mainly GR independent. (A): phosphorylation ratio of AKT at Ser473. (B): phosphorylation ratio of mTOR at Ser2448. (C): phosphorylation ratio of mTOR at Ser2481. (D): phosphorylation levels of TSC2 at Ser939. (E): phosphorylation levels of TSC2 at Ser1462. (F): protein levels of TSC2. (G): representative images of AKT Western blots. $(H)$ : representative images of mTOR Western blots. (I): representative images of TSC2 Western blots. Significant differences between groups within control or mGRKO mice are indicated by asterisks on horizontal lines. Significant differences between control and mGRKO mice per condition are indicated by asterisks or trend above the column. ${ }^{*}=\mathrm{P}<0.05,{ }^{* *}=\mathrm{P} \leq 0.01$ or ${ }^{* * *}=\mathrm{P} \leq 0.001$. Trends $(0.05 \leq \mathrm{P} \leq 0.1)$ are indicated by the specific P-value $(n=6-8)$.

\section{Discussion}

In this study, the contribution of muscle GR signaling to hypoxia-induced muscle atrophy was addressed. As food intake is reduced in mice exposed to hypoxia [21], a pair-fed group was included to assess the GR-dependency of responses induced by hypoxia and by semi-starvation under normoxic conditions. GR deficiency prevented muscle atrophy in pair-fed but not in hypoxic mice. In line with this, GR deficiency significantly attenuated muscle proteolysis signaling in response to reduced food intake. The increased expression of UPS-related genes was mainly independent of GR in muscle of hypoxic mice. Importantly, despite appropriate AKT/mTOR signaling, the suppression of mTOR activity under semistarvation conditions was impaired by hypoxia in a GR-dependent manner.

Fasting-induced muscle atrophy is associated with elevated corticosterone concentrations [31]. In line with this, acute or chronic reduction of food intake in normoxic or hypoxic conditions increases corticosterone concentrations. Mice subjected to hypoxia in our model are hypoxemic [21], and hypoxemia has also been associated with elevated GC concentrations and GR signaling independent of malnutrition [32-36]. However, the absence of consistent additional increases in hypoxic compared to normoxic pair-fed mice suggests the elevated corticosterone concentration is attributable to reduced food intake alone. Muscle GR signaling as revealed by GRE-reporter activation, indicated the circulating corticosterone affected skeletal muscle. In addition, circulating cortisol-although in general $4 \%$ of plasma GC concentrations [37], may contribute to GR-activity in muscle. This may explain the difference between pair-fed and hypoxia GREactivation, which was not reflected by the corticosterone levels. Considering their postulated transcriptional regulation by GR $[17,38]$ the increases in Glul and Foxo1 likely reflect genomic actions of activated GR in response to the raised corticosterone concentrations. Interestingly, in response to hypoxia muscle $\mathrm{Gr}$ mRNA transcript levels increase. However, as increased Glul and Foxo-1 expression and GR-reporter activity do not reveal consistent additive effects of hypoxia compared to reduced food intake, this suggests that the genomic actions of GR in hypoxic muscle are attributable to reduced food intake. 
Although increased by semi-starvation, mRNA expression of the putative GR target genes Klf15 and Redd1 [17,38] is minimally affected by GR deficiency. Furthermore, under hypoxic conditions, increases in KIf15 and Redd1 expression are attenuated (Klf15) or absent (Redd1), indicating that hypoxia blocks transcriptional activation of KIf15 and Redd1 by semi-starvation. KIf15 gene transcription in cardiomyocytes is dependent on intra-cellular calcium and ATP concentrations [39], which may be inadequate to increase KIf15 expression in hypoxic muscle. Although testosterone concentrations were not measured in this study, it has been shown that induction of Redd1 expression by glucocorticoids is abolished in the presence of an elevated testosterone concentration [40]. This may explain the lack of Redd1 transcript accumulation in muscle of hypoxic mice, as $8 \%$ normobaric hypoxia is known to increase serum testosterone concentrations within 1 day and this increase is abrogated at day 5 [41].

Protein degradation in skeletal muscle involves the ubiquitin-proteasome system (UPS) (18) and the autophagy lysosomal pathway (ALP) [43]. Although the MAP1LC3B-I protein levels were reduced, indicative of elevated autophagy, no increase in MAP1LC3B-II was found. The absence of MAP1LC3B-II could be the result of the elevated autophagy and thereby degradation of MAP1LC3B-II itself. Although there are no differences between control and $\mathrm{mGRKO}$ in response of MAPL1LC3B-I and -II levels, hypoxia resulted in lower levels of MAP1LC3BII, which is an indication of elevated autophagy compared to the PF-group. The expression of the ALP-related genes Map1/c3b and Bnip3 is upregulated mainly by the hypoxia-mediated reduction in food intake. GR deficiency blunts this response slightly, but significantly. Interestingly, this corresponds with the GR-dependent induction of Foxo-1, which has been implicated in the transcriptional regulation of ALP-related genes [43], and suggests that genomic actions of GR may indirectly contribute to control of autophagy in muscle. Considering the partially sustained Bnip3 and Map1/c3B expression following hypoxia and semi-starvation, induction of ALP-related gene expression also clearly involves a GR-independent mechanism, which may include ATF4, CHOP [44] or HIF1a [45]. In line with previous studies, increases in Murf1 and Atrogin-1 expression in response to (semi-) starvation require muscle $\mathrm{Gr}$ expression [12]. However, increases in the expression of these atrogenes occur independently of GR under hypoxia, and may contribute to loss of muscle mass. KLF15 is a key regulator of Murf1 and Atrogin-1 expression, and reported to be a direct target gene of GR [17]. Its GR-independent regulation in response to reduced food intake, and its decreased expression in response to hypoxia, however, exclude a key regulatory function of de novo synthesized KLF15 on Murf1 and Atrogin-1 expression under hypoxic conditions. Foxo1 is a target gene of GR and a transcriptional 
regulator of Murf1 and Atrogin-1 [14], and its expression corresponds to the lowered transcript levels of these atrogenes during reduced food intake. However, a GR-independent mechanism that does not require increased Foxo1 expression contributes to atrogene regulation in response to hypoxia. As KLF15 and FOXO1 are not responsible for the increased expression of Murf1 and Atrogin-1 during hypoxia, the transcriptional regulators in play remain to be identified. Overall, GR deficiency attenuates ALP-associated gene expression. Furthermore, genomic actions of GR are involved in upregulation of Murf1 and Atrogin-1 expression during reduced food intake, whereas Atrogin-1 and Murf1 induction under hypoxic conditions occurs independently of GR.

Hypoxia reduces food intake [21], and (semi-)starvation results in the suppression of muscle protein synthesis [46, 47]. mTORC1 activity (based on 4E-BP1-, P70S6K1 phosphorylation ratio) is decreased accordingly in muscle of normoxic control mice in response to reduced food intake. Interestingly, in mGRKO muscle mTORC1 activity is reduced to a similar extent as in pair-fed control animals in response to reduced food intake, indicating that this suppression of mTORC1 activity occurs independently of muscle GR-signaling. This suggests that muscle atrophy in response to semi-starvation is a consequence of GR-dependent proteolysis rather than reduced protein synthesis. In addition to increases in endogenous glucocorticoids, nutritional restriction is accompanied by decreased circulating insulin and IGF-I levels [48]. Although plasma insulin was not measured, the low AKT phosphorylation in muscle of pair-fed control mice suggest that reduced anabolic signaling cues, rather than the increased circulating corticosterone concentration are responsible for the suppression of mTORC1 activity. Dysregulation of muscle protein synthesis signaling in response to acute (4 days) hypoxia was previously described by our group [21], and is characterized by sustained mTORC1 activity in the presence of reduced food intake. We now show that GR is involved in this dysregulation, since in hypoxic mGRKO mice mTORC1 activity is suppressed to levels comparable to pair-fed mice. REDD1 inhibits mTORC1 activity through liberating TSC2 by competing for its binding to 14-3-3 [17]. Redd1 expression has been postulated to be GR-dependent (29). Although decreased mTORC1 activity correlates with increased Redd1 transcript levels under PF conditions, Redd1 expression does not appear to be GR-dependent under acute conditions of reduced food intake. Although an association was found between corticosterone levels and Redd1 expression during fasting [50], although timing and the absolute versus partial withdrawal of food may contribute to these differential results. Nevertheless, the lack of Redd1 induction in response to hypoxia in both control as well as in mGRKO muscle, strongly suggest that Redd1 expression does not control mTORC1 activity in response to hypoxia. KLF15 has 
also been postulated to operate GR-dependently, and to inhibit mTORC1 activity through control of BCAT2 expression [17]. In contrast to in vitro studies in which dexamethasone was used to induce GR/KLF15 signaling [17], KIf15 expression under hypoxia and reduced food intake in vivo was not GR-dependent, and did not correlate inversely with mTORC1 activity. Combined, this indicates that aberrant regulation of Redd1 and KIf15 by hypoxia is not responsible for the sustained mTORC1 activity in conditions of semi-starvation.

Non-genomic actions of GR have also been implicated in mTORC1 signaling by inhibiting AKT phosphorylation through an interaction with PI3K [51, 52]. The decreased AKT phosphorylation ratio in control mice under pair-fed and hypoxic conditions may reflect inhibition of PI3K activity by corticosterone-driven nongenomic GR actions. Although GR deficiency lowers basal AKT phosphorylation, this is not further reduced by either reduced food intake alone or hypoxia. Therefore, the non-genomic action of GR on AKT signaling is not involved in the lack of mTORC1 inhibition under hypoxic conditions. AKT induces mTOR activity through inhibition of TSC2 by phosphorylation at sites Ser939 and Thr1462 [53]. Interestingly, a reduction of TSC2 phosphorylation at sites Ser939 and Thr1462, which corresponds with suppressed 4E-BP1 and S6 phosphorylation, is only observed in pair-fed control animals and resulting from reduced total TSC2 protein levels. Accordingly, the TSC2 phosphorylation status is unchanged in hypoxic mGRKO muscle, where suppression of mTORC1 activity is restored. Therefore alterations in TSC2 phosphorylation are unlikely to contribute to the GR-dependent impairment of mTORC1 signaling. In addition to indirect stimulation of $\mathrm{mTORC} 1$ via TSC2 inhibition, AKT is capable of direct stimulatory mTOR phosphorylation at Ser2448 [28]. AKT and mTOR Ser2448 phosphorylation are coordinately reduced under hypoxic and pair-fed conditions in control animals, suggesting that insulin/AKT signaling cues to control mTORC1 activity are preserved in response to hypoxia. In accordance, mTOR Ser2448 phosphorylation is equally reduced in $\mathrm{mGRKO}$ muscle, further emphasizing that hypoxia-mediated dysregulation of mTORC1 is not the consequence of altered mTOR regulation at site Ser2448. Auto-phosphorylation of mTOR at site Ser2481 has been described and contributes to mTORC1 activity, but its regulation is only partly understood [28]. Interestingly, mTOR Ser2481 phosphorylation does not correlate with the changes in 4E-BP1 and RPS6 phosphorylation in response to reduced food intake. However, only in mGRKO muscle a reduction of mTOR Ser2481 phosphorylation under hypoxia was observed, which corresponds to the restoration of an appropriate response to semi-starvation even in presence of hypoxia. Although the exact mechanism remains to be elucidated, this suggests that GR-dependent preservation of mTOR Ser2481 phosphorylation may contribute to impaired 
mTORC1 regulation during hypoxia. Overall, the GR-dependent impairment of mTORC1 activity regulation in response to hypoxia is neither explained by aberrant upstream AKT/TSC2- or AKT/mTOR-Ser2448 signaling, nor by alterations in Redd1 or Klf15 expression. GR-mediated control of mTOR Ser2481 phosphorylation therefore appears to contribute to the regulation of protein synthesis independently of the established non-genomic actions of GR.

Protein synthesis signaling in muscle of hypoxic, hypophagic mice was comparable to normoxic controls [21], whereas semi-starvation in normoxia reduced mTOR activity as anticipated [53]. Protein synthesis and degradation signaling change in a coordinated fashion in conditions altering muscle mass [55-57]. In line, the decreased protein synthesis signaling in response to semi-starvation is accompanied by an increased expression of genes mediating proteolysis in atrophying muscle. In contrast, the coordination of synthesis and proteolysis signaling cues appears impaired under hypoxia in a GR-dependent manner. The sustained stimulatory signals for protein synthesis signaling are not sufficient to counteract proteolysis signaling inflicted by hypoxia or semi-starvation, as muscle atrophy is equal or even more extensive in response to hypoxia when compared to semistarvation alone. GR deficiency restores the physiological response to reduced food intake, i.e. suppression of $\mathrm{mTORC} 1$ signaling under hypoxic conditions. This does not aggravate muscle atrophy in the presence of sustained increases of proteolytic cues in mGRKO muscle, suggesting that the discordant mTOR activity under hypoxic conditions may reflect futile protein synthesis signaling. In COPD patients with muscle atrophy, molecular signatures reflecting parallel increases in protein synthesis and degradation signaling in skeletal muscle have been reported [58-61], and associated with a hypermetabolic state. This impaired coordination of protein synthetic and proteolytic cues may contribute to the loss of muscle mass observed in pathological conditions.

In conclusion, GR signaling - likely mediating genomic actions - is required for muscle atrophy and increased expression of proteolysis-associated genes induced by decreased food intake under normoxic conditions. However, under hypoxic conditions, muscle atrophy and elevated gene expression of the UPSassociated E3 ligases Murf1 and Atrogin-1 is independent of GR signaling. In addition, hypoxia GR-dependently impairs inhibition of mTORC1 activity in response to reduced anabolic stimuli. The suppression of AKT/mTOR signaling triggered by reduced food intake is intact under hypoxic conditions. This suggests that the lack of mTORC1 inhibition under hypoxic conditions is not dependent on non-genomic GR actions. 


\section{Study limitations}

The mGRKO mouse is a conditional knockout mouse generated using the CRE/ lox system. Cre-recombinase under control of the MLC promoter is expressed only in type-II fibers. The gastrocnemius consists mainly of type II fibers, with less than $6 \%$ of all fibers being of type $I$. This accounts for less than $1 \%$ of the MYHC proteins [62]. The remaining Gr expression observed in the mGRKO mouse in response to hypoxia and reduced food intake is probably due to these fibers and to cells of the vasculature, connective tissue and nerves. Although basal levels of cortisol are low $(\sim 4 \mathrm{ng} / \mathrm{ml})$ versus corticosterone $(\sim 100 \mathrm{ng} / \mathrm{ml})$ it might respond quicker than corticosterone during an acute stress situation [37]. In the current article cortisol was not measured and therefore no indication of the role of cortisol can be shown. Although not included in this study, measurements of muscle protein synthesis and protein degradation rate by hypoxia will assist in further unraveling the mechanisms of hypoxia-induced muscle atrophy.

\section{Acknowledgements}

The GRE-luciferase construct was kindly provided by R. Hoffmann (University Medical Center Groningen, Groningen, The Netherlands) 


\section{References}

1. Gray-Donald K, Gibbons L, Shapiro SH, Macklem PT, Martin JG. Nutritional status and mortality in chronic obstructive pulmonary disease. American journal of respiratory and critical care medicine. 1996;153(3):961-6.

2. Decramer M, De Benedetto F, Del Ponte A, Marinari S. Systemic effects of COPD. Respiratory medicine. 2005;99 Suppl B:S3-10.

3. Jagoe RT, Engelen MP. Muscle wasting and changes in muscle protein metabolism in chronic obstructive pulmonary disease. Eur Respir J Suppl. 2003;46:52s-63s.

4. Mador MJ, Bozkanat E. Skeletal muscle dysfunction in chronic obstructive pulmonary disease. Respir Res. 2001;2(4):216-24.

5. Wouters EF. Nutrition and metabolism in COPD. Chest. 2000;117(5 Suppl 1):274S-80S.

6. Langen RC, Gosker HR, Remels AH, Schols AM. Triggers and mechanisms of skeletal muscle wasting in chronic obstructive pulmonary disease. The international journal of biochemistry \& cell biology. 2013;45(10):2245-56.

7. Chaudhary P, Suryakumar G, Prasad R, Singh SN, Ali S, Ilavazhagan G. Chronic hypobaric hypoxia mediated skeletal muscle atrophy: role of ubiquitin-proteasome pathway and calpains. Mol Cell Biochem. 2012.

8. Hayot M, Rodriguez J, Vernus B, Carnac G, Jean E, Allen D, et al. Myostatin up-regulation is associated with the skeletal muscle response to hypoxic stimuli. Molecular and cellular endocrinology. 2010.

9. de Theije C, Costes F, Langen RC, Pison C, Gosker HR. Hypoxia and muscle maintenance regulation: implications for chronic respiratory disease. Current opinion in clinical nutrition and metabolic care. 2011;14(6):548-53.

10. Medina R, Wing SS, Goldberg AL. Increase in levels of polyubiquitin and proteasome mRNA in skeletal muscle during starvation and denervation atrophy. Biochem J. 1995;307 ( Pt 3):631-7.

11. Wing SS, Goldberg AL. Glucocorticoids activate the ATP-ubiquitin-dependent proteolytic system in skeletal muscle during fasting. The American journal of physiology. 1993;264(4 Pt 1):E668-76.

12. Hu Z, Wang H, Lee IH, Du J, Mitch WE. Endogenous glucocorticoids and impaired insulin signaling are both required to stimulate muscle wasting under pathophysiological conditions in mice. The Journal of clinical investigation. 2009;119(10):3059-69.

13. Stahn C, Buttgereit F. Genomic and nongenomic effects of glucocorticoids. Nature clinical practice Rheumatology. 2008;4(10):525-33.

14. Sandri M, Sandri C, Gilbert A, Skurk C, Calabria E, Picard A, et al. Foxo transcription factors induce the atrophy-related ubiquitin ligase atrogin-1 and cause skeletal muscle atrophy. Cell. 2004;117:399-412. 
15. Jagoe RT, Lecker SH, Gomes M, Goldberg AL. Patterns of gene expression in atrophying skeletal muscles: response to food deprivation. FASEB journal : official publication of the Federation of American Societies for Experimental Biology. 2002;16(13):1697-712.

16. McLoughlin TJ, Smith SM, DeLong AD, Wang H, Unterman TG, Esser KA. FoxO1 induces apoptosis in skeletal myotubes in a DNA-binding-dependent manner. American journal of physiology Cell physiology. 2009;297(3):C548-55.

17. Shimizu N, Yoshikawa N, Ito N, Maruyama T, Suzuki Y, Takeda S-i, et al. Crosstalk between glucocorticoid receptor and nutritional sensor mTOR in skeletal muscle. Cell metabolism. 2011;13:170-82.

18. He Y, Hakvoort TB, Kohler SE, Vermeulen JL, de Waart DR, de Theije C, et al. Glutamine synthetase in muscle is required for glutamine production during fasting and extrahepatic ammonia detoxification. J Biol Chem. 2010;285(13):9516-24.

19. Esbaugh AJ, Walsh PJ. Identification of two glucocorticoid response elements in the promoter region of the ubiquitous isoform of glutamine synthetase in gulf toadfish, Opsanus beta. American journal of physiology Regulatory, integrative and comparative physiology. 2009;297(4):R1075-81.

20. Wang H, Kubica N, Ellisen LW, Jefferson LS, Kimball SR. Dexamethasone represses signaling through the mammalian target of rapamycin in muscle cells by enhancing expression of REDD1. J Biol Chem. 2006;281(51):39128-34.

21. de Theije CC, Langen RC, Lamers WH, Schols AM, Koehler SE. Distinct responses of protein turnover-regulatory pathways in hypoxia- and semi-starvation-induced muscle atrophy. American journal of physiology Lung cellular and molecular physiology. 2013.

22. Bothe GW, Haspel JA, Smith CL, Wiener HH, Burden SJ. Selective expression of Cre recombinase in skeletal muscle fibers. Genesis. 2000;26(2):165-6.

23. Tronche F, Kellendonk C, Kretz O, Gass P, Anlag K, Orban PC, et al. Disruption of the glucocorticoid receptor gene in the nervous system results in reduced anxiety. Nature genetics. 1999;23(1):99-103.

24. Hermans JJ, van Essen H, Struijker-Boudier HA, Johnson RM, Theeuwes F, Smits JF. Pharmacokinetic advantage of intrapericardially applied substances in the rat. The Journal of pharmacology and experimental therapeutics. 2002;301(2):672-8.

25. Mason SR, Ward LC, Reilly PE. Fluorimetric detection of serum corticosterone using highperformance liquid chromatography. Journal of chromatography. 1992;581(2):267-71.

26. Zijlstra GJ, Ten Hacken NH, Hoffmann RF, van Oosterhout AJ, Heijink IH. Interleukin-17A induces glucocorticoid insensitivity in human bronchial epithelial cells. The European respiratory journal. 2012;39(2):439-45.

27. Sandri M. Signaling in muscle atrophy and hypertrophy. Physiology. 2008;23:160-70.

28. Foster KG, Fingar DC. Mammalian target of rapamycin (mTOR): conducting the cellular signaling symphony. J Biol Chem. 2010;285(19):14071-7.

29. Wang X, Proud CG. The mTOR pathway in the control of protein synthesis. Physiology. 2006;21:362-9. 
30. Soliman GA, Acosta-Jaquez HA, Dunlop EA, Ekim B, Maj NE, Tee AR, et al. mTOR Ser2481 autophosphorylation monitors mTORC-specific catalytic activity and clarifies rapamycin mechanism of action. J Biol Chem. 2010;285(11):7866-79.

31. Shavlakadze T, Anwari T, Soffe Z, Cozens G, Mark PJ, Gondro C, et al. Impact of fasting on the rhythmic expression of myogenic and metabolic factors in skeletal muscle of adult mice. American journal of physiology Cell physiology. 2013;305(1):C26-35.

32. Gardner DS, Fletcher AJ, Fowden AL, Giussani DA. Plasma adrenocorticotropin and cortisol concentrations during acute hypoxemia after a reversible period of adverse intrauterine conditions in the ovine fetus during late gestation. Endocrinology. 2001;142(2):589-98.

33. Kodama T, Shimizu N, Yoshikawa N, Makino Y, Ouchida R, Okamoto K, et al. Role of the glucocorticoid receptor for regulation of hypoxia-dependent gene expression. J Biol Chem. 2003;278(35):33384-91.

34. Moncloa F, Donayre J, Sobrevilla LA, Guerra-Garcia R. Endocrine studies at high altitude. II. Adrenal cortical function in sea level natives exposed to high altitudes (4300 metersfor two weeks. The Journal of clinical endocrinology and metabolism. 1965;25(12):1640-2.

35. Larsen JJ, Hansen JM, Olsen NV, Galbo H, Dela F. The effect of altitude hypoxia on glucose homeostasis in men. The Journal of physiology. 1997;504 ( Pt 1):241-9.

36. Leonard MO, Godson C, Brady HR, Taylor CT. Potentiation of glucocorticoid activity in hypoxia through induction of the glucocorticoid receptor. Journal of immunology. 2005;174(4):22507.

37. Gong S, Miao Y-L, Jiao G-Z, Sun M-J, Li H, Lin J, et al. Dynamics and Correlation of Serum Cortisol and Corticosterone under Different Physiological or Stressful Conditions in Mice. PLoS One. 2015;10(2):e0117503.

38. Asada M, Rauch A, Shimizu H, Maruyama H, Miyaki S, Shibamori M, et al. DNA bindingdependent glucocorticoid receptor activity promotes adipogenesis via Kruppel-like factor 15 gene expression. Laboratory investigation; a journal of technical methods and pathology. 2011;91(2):203-15.

39. Bround MJ, Wambolt R, Luciani DS, Kulpa JE, Rodrigues B, Brownsey RW, et al. Cardiomyocyte ATP production, metabolic flexibility, and survival require calcium flux through cardiac ryanodine receptors in vivo. J Biol Chem. 2013;288(26):18975-86.

40. Wu Y, Zhao W, Zhao J, Zhang Y, Qin W, Pan J, et al. REDD1 is a major target of testosterone action in preventing dexamethasone-induced muscle loss. Endocrinology. 2010;151(3):10509.

41. Madrid E, Reyes JG, Hernandez B, Garcia JM, San Martin S, Olivero P, et al. Effect of normobaric hypoxia on the testis in a murine model. Andrologia. 2013;45(5):332-8.

42. Glass DJ. Skeletal muscle hypertrophy and atrophy signaling pathways. The international journal of biochemistry \& cell biology. 2005;37(10):1974-84.

43. Sandri M. Autophagy in skeletal muscle. FEBS letters. 2010;584(7):1411-6. 
44. Rouschop KMA, Beucken TVD, Dubois L, Niessen H, Bussink J, Savelkouls K, et al. The unfolded protein response protects human tumor cells during hypoxia through regulation of the autophagy genes MAP1LC3B and ATG5. Cancer. 2010;120:127-41.

45. Kothari S, Cizeau J, McMillan-Ward E, Israels SJ, Bailes M, Ens K, et al. BNIP3 plays a role in hypoxic cell death in human epithelial cells that is inhibited by growth factors EGF and IGF. Oncogene. 2003;22(30):4734-44.

46. Dunn MA, Houtz SK, Hartsook EW. Effects of fasting on muscle protein turnover, the composition of weight loss, and energy balance of obese and nonobese Zucker rats. J Nutr. 1982;112(10):1862-75.

47. Vendelbo MH, Moller AB, Christensen B, Nellemann B, Clasen BF, Nair KS, et al. Fasting increases human skeletal muscle net phenylalanine release and this is associated with decreased mTOR signaling. PLoS One. 2014;9(7):e102031.

48. O'Sullivan U, Gluckman PD, Breier BH, Woodall S, Siddiqui RA, McCutcheon SN. Insulinlike growth factor-1 (IGF-1) in mice reduces weight loss during starvation. Endocrinology. 1989;125(5):2793-4.

49. Kumari R, Willing LB, Jefferson LS, Simpson IA, Kimball SR. REDD1 (regulated in development and DNA damage response 1) expression in skeletal muscle as a surrogate biomarker of the efficiency of glucocorticoid receptor blockade. Biochemical and biophysical research communications. 2011;412(4):644-7.

50. McGhee NK, Jefferson LS, Kimball SR. Elevated corticosterone associated with food deprivation upregulates expression in rat skeletal muscle of the mTORC1 repressor, REDD1. J Nutr. 2009;139(5):828-34.

51. Leis H, Page A, Ramirez A, Bravo A, Segrelles C, Paramio J, et al. Glucocorticoid Receptor Counteracts Tumorigenic Activity of Akt in Skin through Interference with the Phosphatidylinositol 3-Kinase Signaling Pathway. Molecular endocrinology. 2004;18(2):303-11.

52. Arancibia S, Benitez D, Nunez LE, Jewell CM, Langjahr P, Candia E, et al. Phosphatidylinositol 3-kinase interacts with the glucocorticoid receptor upon TLR2 activation. Journal of cellular and molecular medicine. 2011;15(2):339-49.

53. Manning BD, Tee AR, Logsdon MN, Blenis J, Cantley LC. Identification of the tuberous sclerosis complex-2 tumor suppressor gene product tuberin as a target of the phosphoinositide 3-kinase/akt pathway. Mol Cell. 2002;10(1):151-62.

54. Patel J, McLeod LE, Vries RG, Flynn A, Wang X, Proud CG. Cellular stresses profoundly inhibit protein synthesis and modulate the states of phosphorylation of multiple translation factors. Eur J Biochem. 2002;269(12):3076-85.

55. Rothman S. How is the balance between protein synthesis and degradation achieved? Theor Biol Med Model. 2010;7:25.

56. Bonaldo P, Sandri M. Cellular and molecular mechanisms of muscle atrophy. Dis Model Mech. 2013;6(1):25-39.

57. Schiaffino S, Dyar KA, Ciciliot S, Blaauw B, Sandri M. Mechanisms regulating skeletal muscle growth and atrophy. Febs J. 2013;280(17):4294-314. 
58. Doucet M, Russell AP, Léger B, Debigaré R, Joanisse DR, Caron M-A, et al. Muscle atrophy and hypertrophy signaling in patients with chronic obstructive pulmonary disease. American journal of respiratory and critical care medicine. 2007;176:261-9.

59. Doucet M, Dube A, Joanisse DR, Debigare R, Michaud A, Pare ME, et al. Atrophy and hypertrophy signaling of the quadriceps and diaphragm in COPD. Thorax. 2010;65(11):963-70.

60. Creutzberg EC, Schols AM, Bothmer-Quaedvlieg FC, Wouters EF. Prevalence of an elevated resting energy expenditure in patients with chronic obstructive pulmonary disease in relation to body composition and lung function. Eur J Clin Nutr. 1998;52(6):396-401.

61. Yoneda T, Yoshikawa M, Fu A, Tsukaguchi K, Okamoto Y, Takenaka H. Plasma levels of amino acids and hypermetabolism in patients with chronic obstructive pulmonary disease. Nutrition. 2001;17(2):95-9.

62. Augusto V, Padovani CR, Campos GER. Skeletal muscle fiber types in C57BI6 mice. Braz J morphol Sci. 2004;21(2):89-94. 


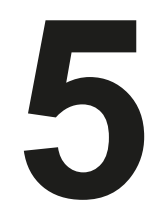

Hypoxia sensitizes skeletal muscle to fasting-induced muscle atrophy and impairs AMPK/mTORC1 signaling in mice

C.C. de Theije, A.M.W.J. Schols, W.H. Lamers, S.E. Köhler, R.C.J. Langen

Submitted 


\section{Abstract}

Background: Acute exacerbations in COPD are often associated with reduced food intake and hypoxemia, which may accelerate muscle wasting in these patients.

Aim: Investigate whether hypoxia sensitizes skeletal muscle to fasting-induced atrophy and affects protein turnover signaling.

Method: Mice were kept under hypoxic $(\mathrm{H})$ ( $8 \%$ oxygen) or normoxic conditions ( $21 \%$ oxygen) $(\mathrm{N})$, or were pair-fed to the hypoxia group (pair-fed normoxic control, PN) for 12 days. Following an additional 24 hrs of fasting, muscle protein turnover signaling was assessed in the gastrocnemius muscle by RT-qPCR and Western blotting.

Results: Weight loss of the gastrocnemius muscle in response to fasting was greater in the hypoxic $(\mathrm{H})$ than the normoxic $(\mathrm{N})$ or pair-fed normoxic control (PN) groups. Conversely, the fasting-induced increase in expression of the ubiquitin 26S-proteasome E3 ligases (Murf1, Atrogin-1) and the autophagy-lysosomal degradation-related genes (Bnip3, Map1/c3B) and protein (LC3B) were attenuated in the $\mathrm{H}$-group compared to the $\mathrm{PN}$-group. mTORC1 activity was reduced by fasting under normoxic conditions but sustained under hypoxic conditions. Fasting-induced inhibition of mTORC1 was accompanied by reduced AKT1/ TSC2 and AKT1/mTOR phosphorylation in the PN-group. Under hypoxic conditions, decreased AKT1/TSC2 and AKT1/mTOR phosphorylation did not match sustained mTORC1 activity. Fasting-induced activation of AMPK/TSC2 signaling correlated with the inhibition of mTORC1 activity in the PN-group. Conversely, no AMPK/TSC2 activation was observed under hypoxic conditions, which correlated with sustained mTORC1 activity.

Conclusion: Fasting-induced AMPK signaling is impaired under hypoxia and is associated with sustained mTORC1 activity, which may sensitize skeletal muscle to fasting-induced muscle atrophy. 


\section{Introduction}

Chronic obstructive pulmonary disease (COPD) is characterized by persistent airflow obstruction resulting from inflammation and remodeling of the airways, and may include the development of emphysema. COPD is an important global health problem as recently shown by the Global Burden of Disease Study 2013 [1]. Disease burden is not only determined by respiratory disease severity, but also by frequency of acute exacerbations and related hospitalization as well as by systemic disease manifestations. Weight loss and muscle wasting are prominent features in particular in the emphysematous [2] phenotype and in patients suffering from chronic hypoxemia [3]. Weight loss and muscle wasting have also been associated with short-term hypoxemic episodes during acute disease exacerbations [4-6]. Prior to hospitalization for acute exacerbations an inverse correlation between the degree of weight loss and resting arterial oxygen tension was reported [7], and upon admission mean dietary intake was decreased by $40 \%$. A slow recovery to normal caloric intake was observed after 4 days hospitalization [8].

An imbalance in muscle protein turnover signaling may result in muscle wasting. The proteasomal and lysosomal pathways play a major role in the degradation of protein in muscle. Rate-determining enzymes of proteasomal protein degradation are E3 ubiquitin ligase tripartite motif containing 63 (TRIM63, MURF1) and F-box protein 32 (FBXO32, ATROGIN-1). Increased expression of these markers of proteasomal protein degradation [9] has been associated with skeletal muscle loss induced by fasting [10-14] and hypoxia [15, 16]. The lysosomal protein markers BCL2/adenovirus E1B 19kDa protein interacting protein 3 (BNIP3) and microtubule-associated protein 1 light chain-3B (MAP1LC3B) are also increased by fasting and hypoxia [17-19]. These protein degradation pathways are regulated through several factors including activating transcription factor 4 (ATF4), CCAAT/-enhancer-binding protein homologous protein (CHOP) [20] or hypoxia-inducible factor $1 \alpha(\mathrm{HIF} 1 \alpha)$ [21] and glucocorticoid receptor (GR)dependent transcriptional regulators, such as Krüppel-like factor 15 (KLF15) [22] and FOXO's [18, 23, 24]. GR also regulates protein synthesis through KLF15 [22] and DNA-damage-inducible transcript 4 protein (DDIT4, a.k.a.REDD1) [25] expression. REDD1 inhibits mammalian target of rapamycin (mTOR) through activation of tuberous sclerosis complex 2 (TSC2) [26]. TSC2 activity can further be controlled by protein kinase B (PKB, a.k.a. AKT1) and AMP-activated protein kinase (AMPK) [27]. Hypoxia [26, 28] and fasting [29, 30] have been shown to inhibit mTORC1 activity.

We hypothesized that hypoxia sensitizes skeletal muscle to fasting-induced atrophy and fasting-induced alterations in protein turnover signaling. To test this hypothesis, mice were first exposed to hypoxia for 12 days, and then subjected to $24 \mathrm{hrs}$ of fasting. A normoxic group that was pair-fed to the hypoxic animals 
was introduced to distinguish effects of hypoxia alone from those of reduced food intake. Protein turnover signaling was assessed in the gastrocnemius muscle by RT-qPCR and Western blot analysis.

\section{Methods}

\section{Animals and tissue collection.}

All mouse studies were carried out according to a protocol approved by the Institutional Animal Care Committee of Maastricht University. C57BL6/J mice were randomly divided into 3 groups: normoxia $(\mathrm{N})$, normobaric hypoxia $(\mathrm{H})$ and normoxia with pair-feeding to the hypoxia group (PN). All mice were housed in experimental chambers at $21^{\circ} \mathrm{C}$ with a 12-hour dark/light cycle. Mice received standard chow (V1534-000 Ssniff R/M-H, Ssniff Spezialdiäten) and water ad libitum. After 5 days of acclimatization, normoxic and pair-fed mice were exposed to $21 \%$ $\mathrm{O} 2$ and hypoxic groups to normobaric hypoxia. Using the proOX system P110 (BioSpherix), ambient oxygen was reduced in a stepwise manner to $12 \%$ (day 1 ), $10 \%$ (day 2$)$, and finally $8 \%(61 \mathrm{mmHg})$ on day 3 , which was then maintained for the remainder of the experiment. To assess the effects of reduced food intake during hypoxia, pair-fed animals received daily the amount of food consumed by the hypoxic mice. After 11 days, body weights and food-intake had been stable for at least 5 days. For the 24-hour-fasting protocol, mice were placed in new cages without food but water ad libitum. Mice were then sacrificed, plasma and gastrocnemius muscle were collected and frozen immediately in liquid nitrogen for further analysis. Muscle weights were corrected for bodyweight at the start of the experiment.

\section{Corticosterone measurement.}

Plasma corticosterone concentrations were determined by HPLC. Cortisol carboxylic acid [31] was added as internal control to the plasma and corticosterone standards. Samples and standards were acidified with phosphoric acid, and steroid hormones were subsequently extracted from plasma with diethyl ether and dried with nitrogen gas. The pellet was dissolved in the eluent (acetonitrile/ methanol/TFA) and incubated with sulphuric acid for 20 minutes to convert the steroids into fluorescent products. Subsequently, HPLC analysis was performed as described previously [32]. All corticosterone values were corrected to the internal standard.

\section{RT-qPCR.}

Total RNA was isolated using TRI REAGENT TM (Sigma-Aldrich) and further purified by precipitation with $2 \mathrm{M} \mathrm{LiCl}$. cDNA synthesis was performed with random hexamer primers on denatured RNA using the Transcriptor First Strand cDNA 
Synthesis Kit (Roche). Real-time quantitative PCR (RT-qPCR) was performed in the iQ5 thermal cycler (Biorad) using the qPCR SyBr Green Fluorescein Mix (Abgene). qPCR primers were designed using Primer Express 2.0 software (Applied Biosystems) and ordered from Sigma Genosys (Table 1). All primers were intron-spanning to avoid contamination of the amplification products with genomic DNA. Expression of genes was normalized to $18 \mathrm{~S}$ ribosomal RNA. RT-qPCR data are normalized to basal mRNA concentrations under normoxic conditions without fasting (day 0 ).

\begin{tabular}{llll}
\hline \hline Gene & NCBI nr. & Forward primer (5' to 3') & Reverse primer (5'to 3') \\
\hline 18S & NR_003278.1 & AGTTAGCATGCCAGAGTCTCG & TGCATGGCCGTTCTTAGTTG \\
Gr & NM_008173 & CGCCAAGTGATTGCCGC & TGTAGAAGGGTCATTTGGTCATCCA \\
Glul & NM_008131.3 & GGCCATGCGGGAGGAGA & GGTGCCTCTTGCTCAGTTTGTCA \\
Fox01 & NM_019739.3 & AAGAGCGTGCCCTACTTCAAG & CCATGGACGCAGCTCTTCTC \\
KIf15 & NM_023184.3 & TGCAGCAAGATGTACACCAAGAG & ATCGCCGGTGCCTTGAC \\
Redd1 & NM_029083.2 & CGGGCCGGAGGAAGACT & CTGCATCAGGTTGGCACACA \\
Murf1 & NM_00103904 & TGTCTGGAGGTCGTTTCCG & CTCGTCTTCGTGTTCCTTGC \\
Atrogin-1 & NM_026346.2 & ACCGGCTACTGTGGAAGAGA & CCTTCCAGGAGAGAATGTGG \\
Map1/c3B & NM_026160.4 & GAGCAGCACCCCACCAAGAT & CGTGGTCAGGCACCAGGAA \\
Bnip3 & NM_009760.4 & CCATGTCGCAGAGCGGG & GACGGAGGCTGGAACGC \\
\hline
\end{tabular}

Table 1. Sequences of primers used for RT-qPCR to assess the expression of the indicated genes.

\section{Western blot.}

Western blot was performed according to procedures previously described [15]. In short, frozen gastrocnemius muscle was ground to powder and lysed. Total protein concentration was determined and $12.5 \mu \mathrm{g}$ was used for Western blotting. Ponceaus staining was used to control and correct for protein loading. The membrane was incubated overnight at $4^{\circ} \mathrm{C}$ with primary antibodies; S6: \#2271, p-S6(Ser235/236): \#4856, 4E-BP1: \#9452, p-4E-BP1(Ser65): \#9451, p-4E-BP1(Thr37/46): \#9459, mTOR: \#2983, p-mTOR(Ser2448): \#2971, p-mTOR(Ser2481): \#2974, Akt: \#9272, p-Akt(Ser374): \#9271, TSC2: \#4308, p-TSC2(Ser939): \#3615, p-TSC2(Thr1462): \#3617, TSC2(Ser1387): \#5584, AMPK: \# 2532, AMPK(Thr172): \#2531 (Cell Signaling Technology). Blots were probed with a horseradish peroxidase-conjugated secondary antibody and visualized using chemiluminescence in a LAS-3000 Luminescent Image analyzer (Fujifilm). Bands were quantified using the AIDA software (Fujifilm). All Western blot data were normalized to basal protein concentrations under normoxic conditions without fasting (day 0 ). 


\section{Statistics.}

Data are shown as means \pm SEM. Comparisons were computed with SPSS version 20 (SPSS Inc., IL, USA). Statistical significance between groups was tested by using the Student's t-test. A P-value of $<0.05$ was considered to be statistically significant and a $\mathrm{P}$-value $0.05 \leq \mathrm{P} \leq 0.1$ as indicating a trend.

\section{Results}

\section{Hypoxia sensitizes skeletal muscle to fasting-induced atrophy.}

Food-intake decreased under hypoxia to $\sim 55 \%$ of the normal intake during the first days and stabilized thereafter at $\sim 81 \%$ of normal intake (Fig. 1A). The decrease of food intake alone resulted in a stable reduction of bodyweight by $8 \%$ as observed in pair-fed normoxic control (PN) compared to normoxic ( $N$ ) animals (Fig. 1B). Hypoxia $(\mathrm{H})$ resulted in $\sim 16 \%$ reduction of bodyweight compared to the $\mathrm{N}$-group. Subsequent fasting for $24 \mathrm{hrs}$ resulted in an additional $\sim 6 \%$ reduction of bodyweight in the $\mathrm{N}$ - and $\mathrm{PN}$-group, whereas this was only $\sim 2 \%$ in the $\mathrm{H}$-group.

A significant decrease of gastrocnemius muscle weight was observed after 12 days in the $\mathrm{H}$-group (Fig. 1C). 24 hrs fasting resulted in muscle weight loss in all three conditions (Fig. 1C, D) and was most pronounced in the H-group (Fig. 1D). Overall, hypoxia sensitized skeletal muscle to fasting-induced atrophy, which is in part accounted for by hypoxia-induced reduction in food-intake. To distinguish the specific effects of hypoxia from those due to preconditioning of paired-feeding, the response to fasting was compared between the $\mathrm{H}$ - and $\mathrm{PN}$-groups, and expressed relative to normoxic baseline levels.

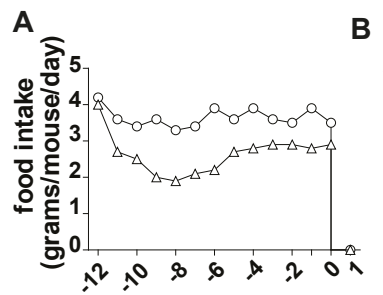

B
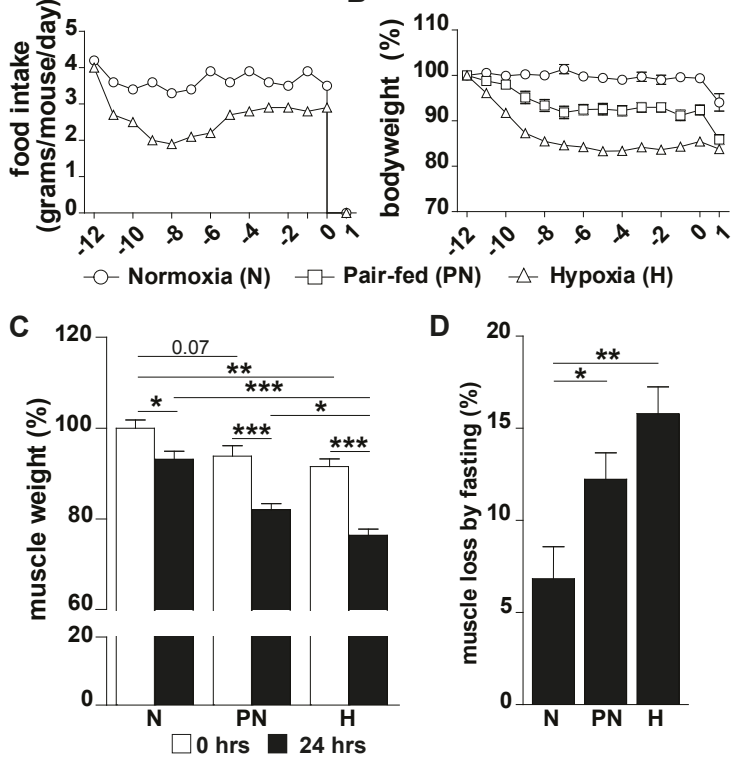

Figure 1. Hypoxia sensitizes skeletal muscle to fasting-induced atrophy. (A): Food intake in gram/ mouse/day per experimental condition. (B): Bodyweights. (C): Muscle weight of gastrocnemius muscle, normalized to the weight of this muscle in normoxic mice (day 0). (D): Loss of muscle mass per condition after $24 \mathrm{hrs}$ of fasting (expressed relative to mass before fasting; day 0 ). Significant differences between groups at a given time point are indicated by asterisks, with * $\mathrm{P}<0.05,{ }^{* *}=\mathrm{P} \leq 0.01$ or ${ }^{* * *}=\mathrm{P} \leq 0.001$. Trends $(0.05 \leq \mathrm{P} \leq 0.1)$ are indicated by the specific $P$-value $(n=6-8)$. 
GR response to fasting is blunted under hypoxic conditions.

To investigate the effect of fasting under normoxic and hypoxic conditions on glucocorticoid signaling, we measured plasma corticosterone (cc) concentrations (Fig. 2A). Corticosterone baseline concentrations were slightly elevated in both $\mathrm{PN}$ - and $\mathrm{H}$-groups relative to the $\mathrm{N}$-group $(\mathrm{N}: 0.17 \mu \mathrm{M}, \mathrm{PN}: 0.25 \mu \mathrm{M} \mathrm{P}<0.05, \mathrm{H}$ : $0.29 \mu \mathrm{M} P<0.001)$. Subsequent fasting clearly induced a similar cc release in both $\mathrm{PN}$ - and $\mathrm{H}$-groups. $\mathrm{Gr}$ mRNA expression was equally elevated during fasting in both groups (Fig. 2B). Increased GR activation by fasting was suggested by increased expression of postulated downstream targets of GR in the N-group, namely Glul, Foxo1, Klf15 and Redd1 (Fig. 2C-F). Under hypoxic conditions, Foxo1, Klf15 and Glul expression was blunted in response to fasting, whereas Redd1 expression did not increase at all. Overall, the GR response to fasting was blunted under hypoxic conditions compared to the $\mathrm{PN}$-group despite equally increased cc plasma concentrations.
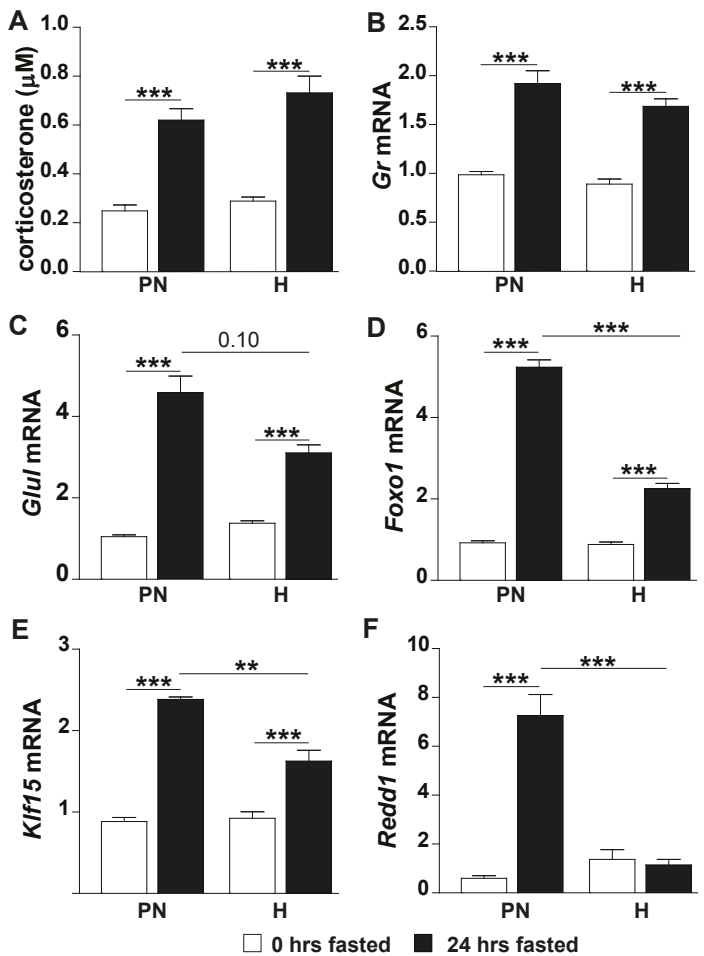

Figure 2. GR response to fasting was blunted under hypoxic conditions. (A): Corticosterone concentrations in plasma. (B): Expression of Gr mRNA. Expression of GR target genes: (C) Glul, (D) Foxo1, (E) Klf15 and (F) Redd1. Data are normalized to the basal expression of the normoxic group at day 0 . Significant differences between groups are indicated by asterisks, with ${ }^{*}=P<0.05,{ }^{* *}=P \leq 0.01$ or ${ }^{* * *}=P \leq 0.001$. Trends $(0.05 \leq P \leq 0.1)$ are indicated by the specific $P$-value $(n=6-8)$. 


\section{Hypoxia blunts fasting-induced protein degradation signaling.}

In order to investigate protein degradation signaling in the gastrocnemius muscle, expression of effectors of the ubiquitin 26S-proteasome system (Atrogin-1, Murf1) and the autophagy lysosomal pathway (Bnip3 and Map1/c3B) were measured. Fasting increased gene expression of Atrogin-1, Murf1, Bnip3 and Map1/c3B in the PN-group (Fig. 3A-D). However, despite extensive muscle atrophy, hypoxia significantly blunted the fasting-induced expression of these same genes. To confirm the blunted response of expression of genes of the autophagy lysosomal pathway at the protein level, the conversion of LC3B from type I to II was investigated (Fig. 3E). Fasting reduced LC3B-I and increased LC3B-II, resulting in an elevated LC3B II / I ratio in the PN-group. In contrast, no changes in LC3B ratios were found in the H-group. The UNC51-like kinase ULK1 induces autophagy through interaction with AMPK. This interaction is inhibited by mTORC1 through phosphorylation at Ser757. To check if ULK1 phosphorylation status was affected by hypoxia or reduced food intake, we measured ULK1 phosphorylation at Ser757 (Fig. 3F). Fasting increased ULK1 protein in the fasting PN-group, although ULK1 Ser757 phosphorylation was still lowered indicating ULK1 activation and upregulation of the autophagy lysosomal pathway. In contrast, under hypoxic condition ULK1 was not induced and fasting did not affect ULK1 Ser757 phosphorylation, indicating that the autophagy lysosomal pathway was not induced as also indicated by ratio of LC3B.
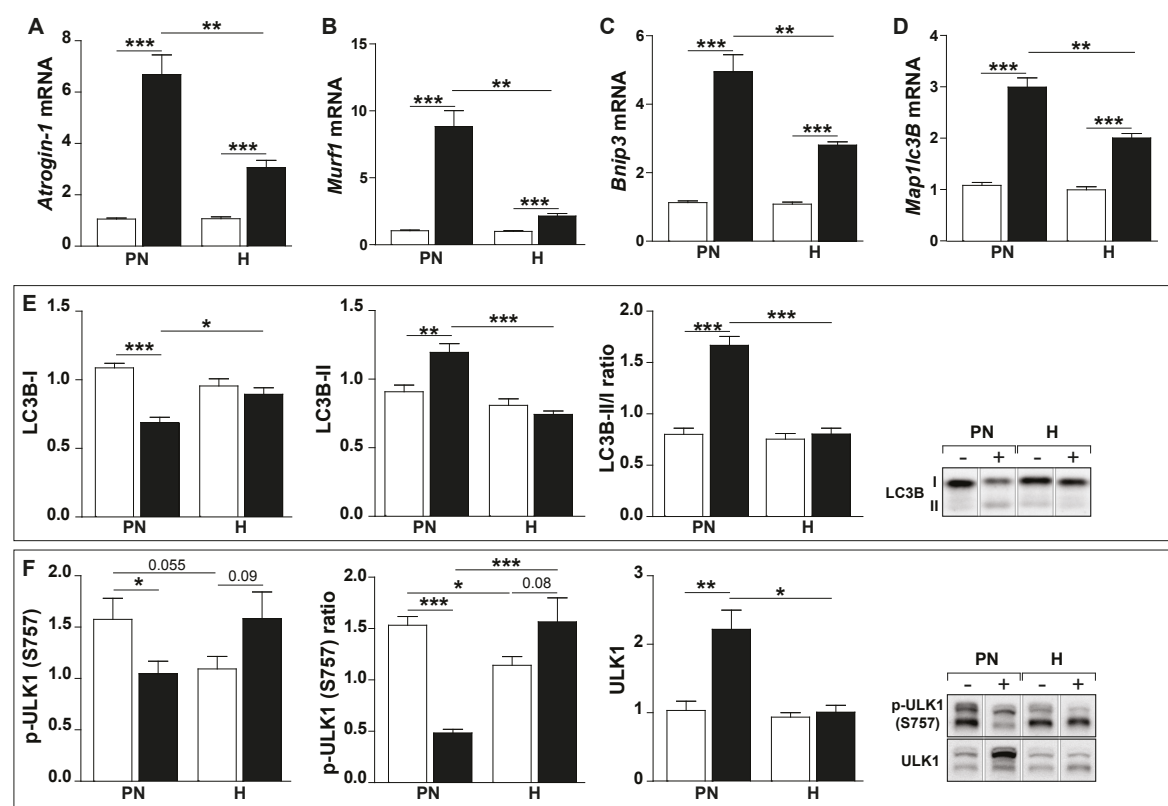

$\square 0$ hrs fasted $\quad 24$ hrs fasted 
Figure 3. Hypoxia blunts fasting-induced protein degradation signaling. Expression of UPSassociated genes: (A) Atrogin-1, (B) Murf1. Expression of ALP-associated genes: (C) Bnip3, (D) Map1/c3B. (E) panel 1: Quantification of LC3B-I, panel 2: Quantification of LC3B-II, panel 3: ratio of LC3B-II over I, panel 4: representative images of LC3B Western blots. (F) panel 1: Quantification of ULK1 Ser757 phosphorylation, panel 2: ratio of p-ULK1 Ser757 over total ULK1, panel 3: total ULK1, panel 4: representative images of ULK1 Western blots. Data are normalized to the basal expression of the normoxic group at day 0 . Significant differences between groups are indicated by asterisks, with ${ }^{*}=\mathrm{P}<0.05,{ }^{* *}=\mathrm{P} \leq 0.01$ or ${ }^{* * *}=\mathrm{P} \leq 0.001$. Trends $(0.05 \leq \mathrm{P} \leq 0.1)$ are indicated by the specific $P$-value $(n=6-8)$.

\section{Hypoxia blocks fasting-induced inhibition of mTORC1.}

Hypoxia and paired feeding (PN) increased mTORC1 activity equally before fasting, as illustrated by increased phosphorylation and increased ratios of phosphorylated over total ribosomal protein S6 (RPS6) and eukaryotic translation initiation factor 4E binding protein (EIF4EBP1, a.k.a.4E-BP1) (Fig. 4A, B). Fasting in the PN-group slightly increased total 4E-BP1 protein concentration and strongly reduced phosphorylation of RPS6 and 4E-BP1. In contrast, fasting under hypoxic conditions did not result in the dephosphorylation of either RPS6 or 4E-BP1. Overall, based on RPS6 and 4E-BP1 phosphorylation, mTORC1 activity is inhibited by fasting under normoxic but not hypoxic conditions.

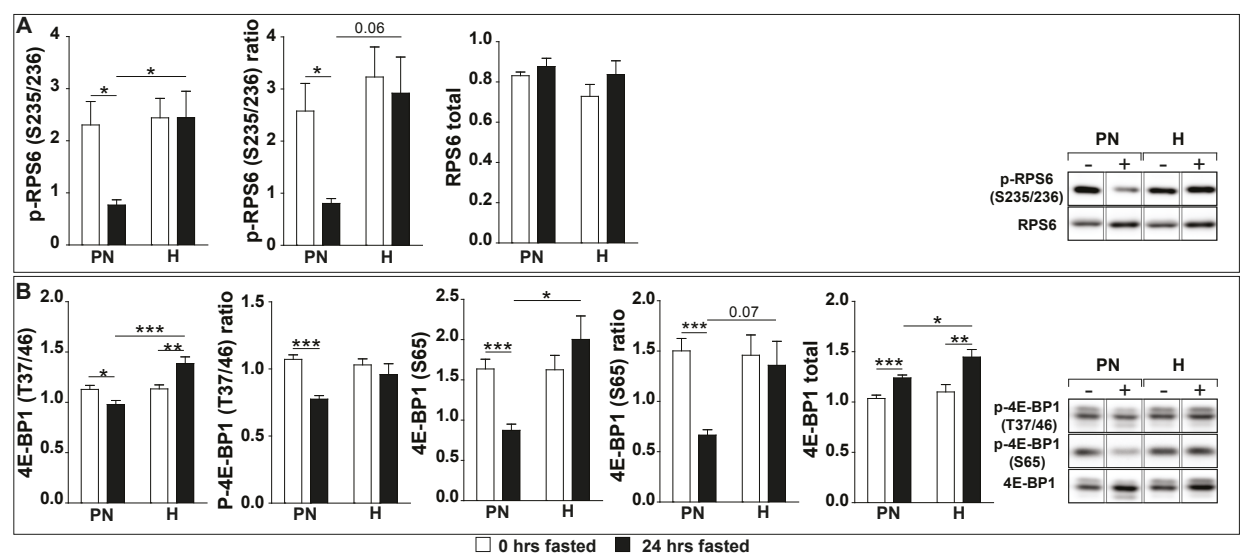

Figure 4. Hypoxia blocks fasting-induced inhibition of mTORC1. (A) panel 1: Quantification of RPS6 Ser235/236 phosphorylation, panel 2: ratio of p-RPS6 (Ser235/236) over total RPS6, panel 3: total RPS6, panel 4: representative images of RPS6 Western blots. (B) panel 1: Quantification of 4E-BP1 Thr37/46 phosphorylation, panel 2: ratio of p-4E-BP1 (Thr37/46) over total 4E-BP1, panel 3: Quantification of 4E-BP1 S65 phosphorylation, panel 4: ratio of p-4E-BP1 (Ser65) over total 4E-BP1, panel 5: total 4E-BP1, panel 6: representative images of 4E-BP1 Western blots. Data are normalized to the basal expression of the normoxic group at day 0 . Significant differences between groups are indicated by asterisks, with ${ }^{*}=\mathrm{P}<0.05,{ }^{* *}=\mathrm{P} \leq 0.01$ or ${ }^{* * *}=\mathrm{P} \leq 0.001$. Trends $(0.05 \leq \mathrm{P} \leq 0.1)$ are indicated by the specific $P$-value $(n=6-8)$. 
mTORC1 activity is uncoupled from AKT1/TSC2 and AKT1/mTOR signaling under hypoxic conditions.

mTORC1 activity is controlled by multiple upstream signaling pathways, including inhibition through the TSC1/2 complex [33]. The TSC1/2 complex itself is inhibited through phosphorylation of Ser939 and Thr1462 on TSC2 by AKT1 [34, 35]. At baseline, AKT1 Ser473 was highly phosphorylated (active) in the PNgroup, while AKT1 activity was significantly lowered in the H-group (Fig. 5A). Subsequent fasting resulted in reduced AKT1 phosphorylation in the PN-group, whereas no further reduction was observed under hypoxic conditions but a trend ( $p=0.053$ ) towards increased phosphorylation. TSC2 Ser939 phosphorylation did not differ between the PN- and H-groups and did not change with fasting in either group (Fig. 5B). In contrast, TSC2 Thr1462 phosphorylation was clearly reduced by hypoxia, and by fasting in the pair-fed group. Fasting did not further decrease TSC2 Thr1462 phosphorylation in the hypoxic mice. TSC2 Thr1462 phosphorylation corresponded to AKT1 Ser473 phosphorylation. AKT1 also phosphorylates mTOR at Ser2448, which may be followed by mTOR Ser2481 autophosphorylation. In line with AKT1 activation, basal phosphorylation of mTOR Ser2448 and Ser2481 were increased in the PN-group, whereas basal levels in the $\mathrm{H}$ group were lower compared to PN (Fig. 5C). Fasting reduced mTOR Ser2448 and Ser2481 phosphorylation in the PN- but not the H-group. Overall, hypoxia suppressed basal AKT1/TSC2 and AKT1/mTOR signaling, and fasting had no additional effect. This implies other upstream regulatory cues involved in the lack of mTORC1 inactivation upon starvation under hypoxic conditions.

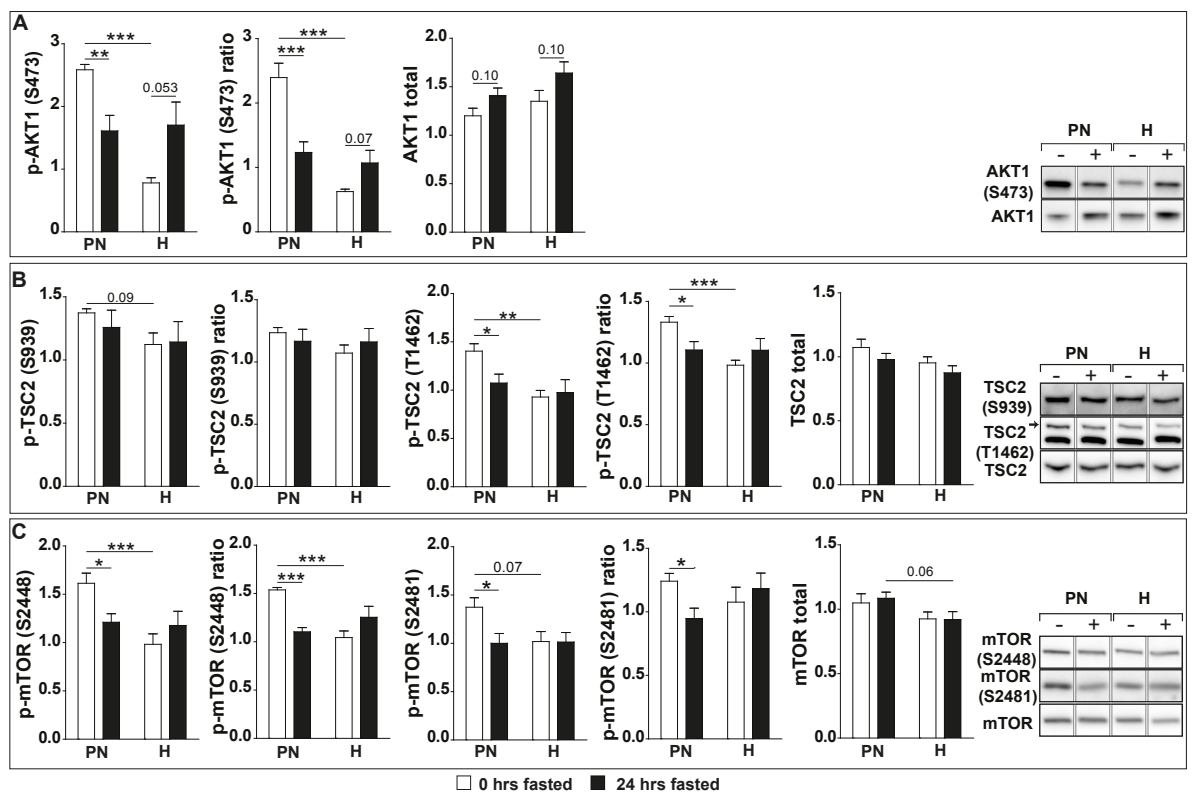


Figure 5. Fasting-induced attenuation of AKT1/TSC2 and AKT1/mTOR signaling does not correlate with mTORC1 activity under hypoxic conditions. (A) panel 1: Quantification of AKT1 Ser473 phosphorylation, panel 2: ratio of p-AKT1 (Ser473) over total AKT1, panel 3: total AKT1, panel 4: representative images of AKT1 Western blots. (B) panel 1: Quantification of TSC2 Ser939 phosphorylation, panel 2: ratio of p-TSC2 (Ser939) over total TSC2, panel 3: Quantification of TSC2 Thr1462 phosphorylation, panel 4: ratio of p-TSC2 (Thr1462) over total TSC2, panel 5: total TSC2, panel 6: representative images of TSC2 Western blots. (C): panel 1: Quantification of mTOR Ser2448 phosphorylation, panel 2: ratio of p-mTOR (Ser2448) over total mTOR, panel 3: Quantification of mTOR Ser2481 phosphorylation, panel 4: ratio of p-mTOR (Ser2481) over total mTOR, panel 5: total mTOR, panel 6: representative images of mTOR Western blots. Data are normalized to the basal expression of the normoxic group at day 0 . Significant differences between groups are indicated by asterisks, with ${ }^{*}=P<0.05,{ }^{* *}=P \leq 0.01$ or ${ }^{* *}=P \leq 0.001$. Trends $(0.05 \leq P \leq 0.1)$ are indicated by the specific P-value $(n=6-8)$.

Fasting-induced control of mTORC1 activity by AMPK/TSC2 signaling is impaired by hypoxia.

mTORC1 activity is inhibited by TSC2 through activation of the AMPK/TSC2 pathway [27]. Phosphorylation of AMPK at site Thr172 is indicative of AMPK activity. Fasting increased AMPK Thr72 phosphorylation in the PN-group, and this response was absent under hypoxic conditions (Fig. 6A). Basal levels of TSC2 Ser1387 were similar to control $(\mathrm{N})$ in both the PN- and the H-group (Fig. 6B). Fasting resulted in increased phosphorylation of TSC2 Ser1387 in the PN- but not the H-group, reflecting the changes observed in AMPK Thr172 phosphorylation. Overall, the responses of the AMPK/TSC2 pathway to fasting in the PN- and $\mathrm{H}$-group corresponded with mTORC1 activity, i.e. a decrease in the PN- but not the H-group.

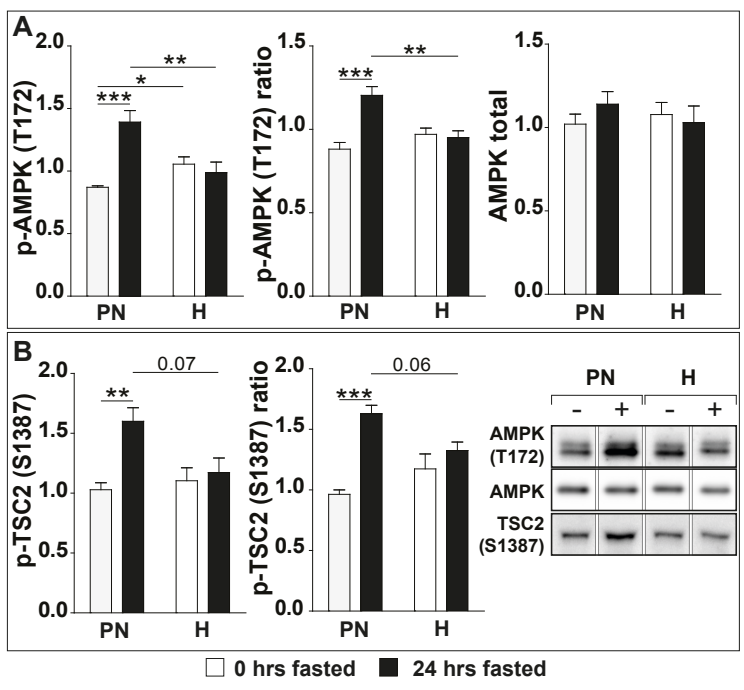

Figure 6. Fasting-induced control of mTORC1 activity by AMPK/TSC2 signaling is impaired by hypoxia. (A) panel 1: phosphorylation of AMPK at Thr172, panel 2: ratio of p-AMPK (Thr172) over total AMPK, panel 3: total AMPK. (B): panel 1: phosphorylation of TSC2 at Ser1387, panel 2: ratio of p-TSC2 (Ser1387) over total TSC2 (for total TSC2 see Fig. 5B panel 6), panel 3: representative images of AMPK and TSC2 Western blots. Data are normalized to the basal expression of the normoxic group at day 0 . Significant differences between groups at a given time point are indicated by asterisks, with ${ }^{*}=\mathrm{P}<0.05$, ${ }^{* *}=\mathrm{P} \leq 0.01$ or ${ }^{* * *}=\mathrm{P} \leq 0.001$. Trends $(0.05 \leq P \leq 0.1)$ are indicated by the specific $P$-value $(n=6-8)$. 


\section{Discussion}

This study shows that hypoxia impairs the adaptation of muscle protein turnover signaling in response to fasting as summarized in Figure 7 . Induction of protein degradation signaling by fasting is blunted in skeletal muscle of hypoxic mice. The fasting-induced increase of the expression of GR target genes was reduced and that of Redd1 was even completely abolished under hypoxic conditions. The latter may contribute to impaired control of mTORC1 during hypoxia. Specifically, fasting-induced inhibition of mTORC1 is absent under hypoxic conditions. The AKT1/TSC2 and AKT1/mTOR axes are intact under hypoxic conditions, since both AKT1 phosphorylation and AKT1-mediated inhibitory phosphorylation of TSC2 are decreased, but this did not result in mTORC1 inhibition. Fasting has no additional effect on AKT1/TSC2 and the AKT/mTOR axis or mTORC1 activity in the hypoxic mice. The AMPK/TSC2-mediated control of mTORC1 activity appeared defective, as under hypoxic conditions the fasting-induced activation of this inhibitory pathway, i.e. increased phosphorylation of AMPK (Thr172) and TSC2 (Ser1387) is absent.

Previously, we and others showed that 4 and 21 days of hypoxia resulted in loss of muscle mass which was partially due to the reduced food intake [15, 36, 37]. In the current study, 12 days of hypoxia or pair-feeding under normoxia induced a comparable muscle loss. Fasting led to a significantly larger muscle loss in the hypoxic group compared to the normoxic group, and this could only partially be explained by the initial reduction in food intake. The more pronounced overall loss of muscle mass after fasting in the H-group compared to the PN-group implies that hypoxia sensitizes skeletal muscle to fasting-induced atrophy.

In line with previous studies by other groups [11, 12, 38, 39], the expression of ALP and UPS-related genes is increased by fasting under normoxic conditions. Increased expression of these effectors of proteolysis is accompanied by increased protein degradation in response to fasting [40,41]. Surprisingly, despite the accentuated muscle mass loss, hypoxia attenuates fasting-induced increases of mRNA transcript levels of UPS and ALP effectors. A limitation of this study is that protein synthesis and proteolysis activity were not measured. However, based on the LC3B data, autophagy is upregulated by fasting but not under hypoxic conditions. Klf15 [22, 42] and Foxo1 [10, 24, 43] have been implicated in the regulation of the ALP and UPS responses, and are targets of GR signaling. Since the fasting-induced increase of the expression of KIf15 and Foxo1 is blunted under hypoxic conditions, GR signaling may be responsible for the observed attenuation of the proteolytic response. However, fasting increased muscle GR expression or plasma corticosterone concentrations to a similar extent in pair-fed 
normoxic control and hypoxic mice. Fasting-induced binding of GR to the glucocorticoid receptor element (GRE) sites within the promoters of the target genes may be affected and result in the observed blunted response. Mechanisms by which hypoxia could inhibit GR signaling include upregulation of a GR inhibitory complex, or inhibition of GR dimerization. It has been shown that FKBP51/52 and P23 recruitment to HSP90 can inhibit GR translocation to the nucleus and thereby transcriptional activity $[44,45]$. Accordingly, hypoxia has been shown to increase constituents of GR inhibitory complexes, HSP90 [46], HSP70 [47, 48] and P23 [49]. Although increased by fasting, the blunted UPS and ALP response contrast the additional muscle loss observed under hypoxic conditions. This suggests that a perturbation of protein synthesis may account for the additional loss of muscle mass.

It has been postulated that muscle atrophy is the consequence of suppressed protein synthesis through GR-dependent regulation of Klf15 [22] and Redd1 [25] expression. As previously reported by our group, acute hypoxia (4 days) blocked the inhibition of mTORC1 by reduced food intake [15]. In the current study, food intake was again stabilized after 8-9 days of hypoxia, and reached $>80 \%$ of the basal food intake. Basal mTORC1 activity was slightly increased in the PN- and $\mathrm{H}$-groups. As expected, fasting inhibits muscle mTORC1 activity in pair-fed animals under normoxic conditions [29, 41, 50]. However, under hypoxic conditions mTORC1 activity is not reduced by fasting. REDD1 can inhibit mTORC1 activity by preventing binding of TSC2 to 14-3-3 [26]. Fasting upregulates Redd1 expression within 12 hours in mice [51], and this corresponds to the Redd1 induction we observed in the fasted normoxic group. Interestingly, in both normoxic and hypoxic animals, fasting-induced changes of Redd1 expression correlated inversely with mTORC1 activity as assessed by the 4E-BP1 and S6 phosphorylation status. This suggests that altered REDD1 regulation by hypoxia may be responsible for the impaired control of mTORC1 activity. Redd1 is considered to be a GR-dependent gene, which is required for glucocorticoid induced muscle atrophy [52]. We previously showed that GR is involved in deregulation of mTORC1 activity under hypoxic conditions, however, we did not observe GRdependency of Redd1 expression in that study (chapter 4).

Insulin and insulin-like growth factor-I are known to influence mTOR activity via the AKT1/TSC2 and AKT1/mTOR pathways. Phosphorylated active AKT1 deactivates TSC2 through phosphorylation at Ser939 and Thr1462, which results in binding of TSC2 to 14-3-3 [34, 35]. The fasting-induced reduction AKT1 phosphorylation observed in $\mathrm{PN}$ and $\mathrm{H}$ mice has been previously reported under normoxic conditions [23] where it corresponded to the fasting-induced decrease of 
the serum insulin concentration $[53,54]$. Since 4 weeks of hypoxia also reduce serum insulin concentrations in mice [55], this may explain the reduced AKT1 phosphorylation under hypoxia observed in this study. We also found that AKT1 and TSC2 Thr1462 phosphorylation were reduced in the H-group compared to the PN-group, and this did not correlate with mTORC1 activity. Fasting did not have an additional affect. In conclusion, AKT1/TSC2 and AKT1/mTOR signaling are not responsible for the deregulation of $\mathrm{mTORC} 1$ activity under hypoxic conditions.

AMPK is a sensor of the energy status of a cell [56]. AMPK can regulate mTORC1 activity through activation of TSC2 through phosphorylation of Ser1387 and Thr1227 [27]. Hypoxia has been shown to increase AMPK activity in vitro [57, 58]. In our mouse model, however, basal AMPK phosphorylation was not increased after 12 days of hypoxia or by fasting under hypoxic conditions. That AMPK responses to hypoxia differ between cultured cells and tissues in vivo, may be due to the absence of systemic effects in vitro. Hypoxia-induced secretion of effector molecules that influence muscle protein turnover, such as corticosterone would be such a systemic effect. Lack of AMPK activation by hypoxia has also been demonstrated in older mice (24 months), although basal levels were already higher [59]. A lack of AMPK response has also been correlated to elevated AKT1 signaling and suppressed LKB1 activity in tumor cell lines and virus-infected cells $[60,61]$. In this study, however, the impaired AMPK response was accompanied by reduced AKT1 activity. Under normoxic conditions, fasting results in increased AMPK phosphorylation and inhibition of mTORC1 activity. However, under hypoxic conditions, fasting did not increase AMPK phosphorylation. Hypoxia results in elevated levels of reactive oxygen species (ROS) in skeletal muscle [62] and it has been demonstrated that ROS production can block fasting-mediated AMPK activation [63]. In addition to ROS, IMP/AMP/ADP/ATP concentrations may regulate AMPK activation. Both elevated IMP but unchanged AMP, ADP and ATP levels [64] and increased AMP degradation to IMP and ammonia during exercise have been shown in COPD patients [65]. Interestingly, hypoxemia $(11 \%$ ambient $\mathrm{O} 2$ ) resulted in increased IMP and stable AMP concentrations in human skeletal muscle during submaximal exercise [66]. Together, these findings suggest that a lack of AMP accumulation due to increased conversion to IMP under hypoxia may impair AMPK activation in response to fasting. AMPK has also been implicated in transcriptional regulation of genes by phosphorylating the CAMP response element-binding (CREB) and inhibition of the transcription factors sterol regulatory element-binding protein 1c (SREBP1c) and carbohydrate response element binding protein (ChREBP) [67]. Recently it has been shown that AMPK activation is linked to increase REDD1 protein levels during hypoxia in head 
and neck squamous cell carcinoma (HNSCC) cells [68]. Such an involvement of AMPK in Redd1 expression may also operate in skeletal muscle, as AMPK activation and Redd1 mRNA transcript levels clearly correlate. AMPK activation has also been implicated in the control of muscle protein turnover by increasing the expression of the atrogenes Murf1 and Atrogin-1 [69, 70]. AICAR and metformin, agonists of AMPK, are able to increase Murf1 and Atrogin-1 expression in C2C12 cells [71]. In addition, AMPK affects autophagy regulation by phosphorylation and activation of ULK1 and FOXO3A [72-74]. The fact that both the increase of AMPK phosphorylation and the induction of the UPS and ALP response by fasting are inhibited under hypoxic conditions, suggests that fasting-induced proteolytic signaling may in part rely on AMPK. Thus, AMPK is a key regulator of signaling pathways involved in protein degradation and synthesis signaling pathways. Combined, we propose that the response of this key regulator to fasting, is impaired under hypoxic conditions (Fig. 7).

\section{Conclusion}

Hypoxia-induced reduction in food intake and hypoxia sensitize skeletal muscle to fasting-induced muscle atrophy. Under hypoxic conditions, fasting results in sustained mTORC1 signaling through impaired AMPK signaling. Induction of UPS and ALP signaling by fasting under hypoxic conditions were blunted or absent. The altered protein synthesis regulation with the blunted protein degradation may account for increased protein turnover and for the additional loss of muscle mass.

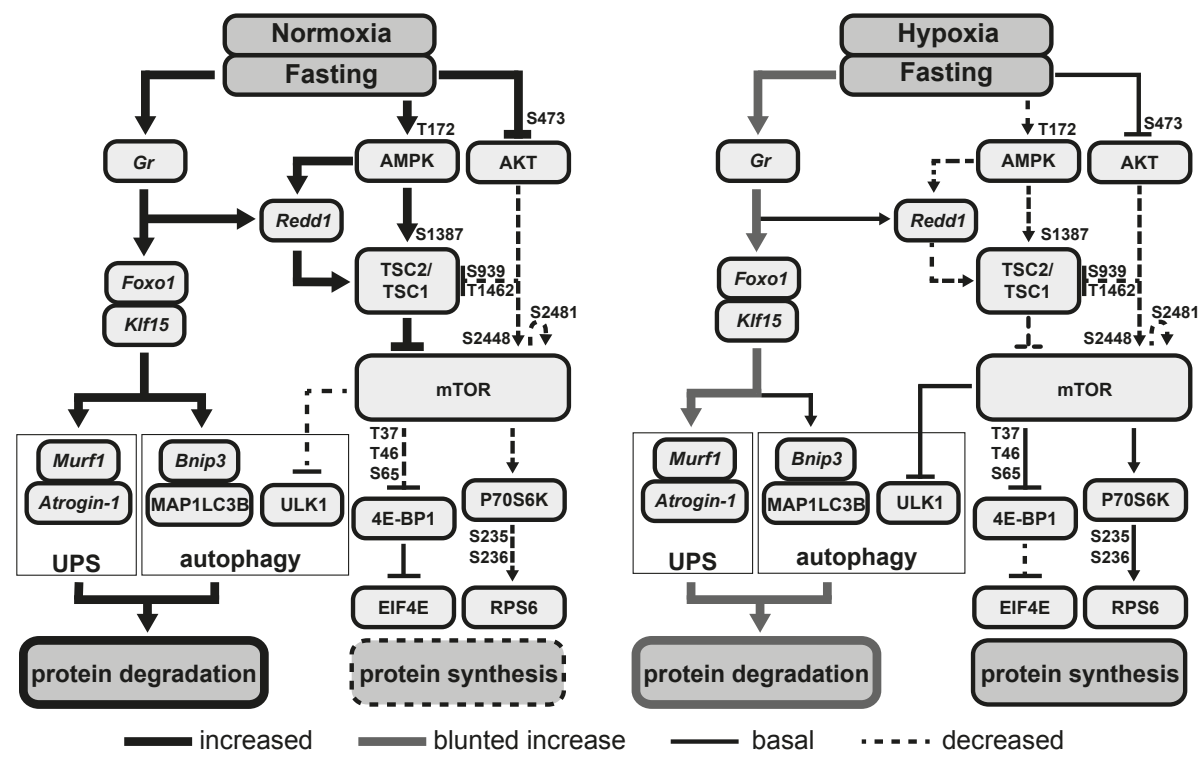

Figure 7. The effects of hypoxia and fasting on protein turnover signaling. Targets measured in this study by mRNA expression are indicated by italics. All shown proteins and their indicated phosphorylation sites were measured in this study (except EIF4E). 


\section{References}

1. Mortality GBD, Causes of Death C. Global, regional, and national age-sex specific all-cause and cause-specific mortality for 240 causes of death, 1990-2013: a systematic analysis for the Global Burden of Disease Study 2013. Lancet. 2014.

2. Engelen MP, Schols AM, Lamers RJ, Wouters EF. Different patterns of chronic tissue wasting among patients with chronic obstructive pulmonary disease. Clinical nutrition. 1999;18(5):27580.

3. Kent BD, Mitchell PD, McNicholas WT. Hypoxemia in patients with COPD: cause, effects, and disease progression. International journal of chronic obstructive pulmonary disease. 2011;6:199-208.

4. Soguel Schenkel N, Burdet L, de Muralt B, Fitting JW. Oxygen saturation during daily activities in chronic obstructive pulmonary disease. The European respiratory journal. 1996;9(12):2584-9.

5. Vestbo J, Hurd SS, Agusti AG, Jones PW, Vogelmeier C, Anzueto A, et al. Global strategy for the diagnosis, management, and prevention of chronic obstructive pulmonary disease: GOLD executive summary. American journal of respiratory and critical care medicine. 2013;187(4):347-65.

6. Barbera JA, Roca J, Ferrer A, Felez MA, Diaz O, Roger N, et al. Mechanisms of worsening gas exchange during acute exacerbations of chronic obstructive pulmonary disease. The European respiratory journal. 1997;10(6):1285-91.

7. Vermeeren MA, Wouters EF, Geraerts-Keeris AJ, Schols AM. Nutritional support in patients with chronic obstructive pulmonary disease during hospitalization for an acute exacerbation; a randomized controlled feasibility trial. Clinical nutrition. 2004;23(5):1184-92.

8. Vermeeren MA, Schols AM, Wouters EF. Effects of an acute exacerbation on nutritional and metabolic profile of patients with COPD. The European respiratory journal. 1997;10(10):22649.

9. Glass DJ. Skeletal muscle hypertrophy and atrophy signaling pathways. The international journal of biochemistry \& cell biology. 2005;37(10):1974-84.

10. Sandri M, Sandri C, Gilbert A, Skurk C, Calabria E, Picard A, et al. Foxo transcription factors induce the atrophy-related ubiquitin ligase atrogin-1 and cause skeletal muscle atrophy. Cell. 2004;117:399-412.

11. Jagoe RT, Lecker SH, Gomes M, Goldberg AL. Patterns of gene expression in atrophying skeletal muscles: response to food deprivation. FASEB journal : official publication of the Federation of American Societies for Experimental Biology. 2002;16(13):1697-712.

12. Gomes MD, Lecker SH, Jagoe RT, Navon A, Goldberg AL. Atrogin-1, a muscle-specific F-box protein highly expressed during muscle atrophy. Proc Natl Acad Sci U S A. 2001;98(25):14440-5.

13. Graca FA, Goncalves DA, Silveira WA, Lira EC, Chaves VE, Zanon NM, et al. Epinephrine depletion exacerbates the fasting-induced protein breakdown in fast-twitch skeletal muscles. Am J Physiol Endocrinol Metab. 2013;305(12):E1483-94. 
14. Shavlakadze T, Anwari T, Soffe Z, Cozens G, Mark PJ, Gondro C, et al. Impact of fasting on the rhythmic expression of myogenic and metabolic factors in skeletal muscle of adult mice. American journal of physiology Cell physiology. 2013;305(1):C26-35.

15. de Theije CC, Langen RC, Lamers WH, Schols AM, Koehler SE. Distinct responses of protein turnover-regulatory pathways in hypoxia- and semi-starvation-induced muscle atrophy. Am J Physiol Lung Cell Mol Physiol. 2013.

16. Chaudhary P, Suryakumar G, Prasad R, Singh SN, Ali S, Ilavazhagan G. Chronic hypobaric hypoxia mediated skeletal muscle atrophy: role of ubiquitin-proteasome pathway and calpains. Mol Cell Biochem. 2012.

17. Bellot G, Garcia-Medina R, Gounon P, Chiche J, Roux D, Pouysségur J, et al. Hypoxiainduced autophagy is mediated through hypoxia-inducible factor induction of BNIP3 and BNIP3L via their BH3 domains. Mol Cell Biol. 2009;29:2570-81.

18. Sandri M. Autophagy in skeletal muscle. FEBS letters. 2010;584(7):1411-6.

19. Bonaldo P, Sandri M. Cellular and molecular mechanisms of muscle atrophy. Dis Model Mech. 2013;6(1):25-39.

20. Rouschop KMA, Beucken TVD, Dubois L, Niessen H, Bussink J, Savelkouls K, et al. The unfolded protein response protects human tumor cells during hypoxia through regulation of the autophagy genes MAP1LC3B and ATG5. Cancer. 2010;120:127-41.

21. Kothari S, Cizeau J, McMillan-Ward E, Israels SJ, Bailes M, Ens K, et al. BNIP3 plays a role in hypoxic cell death in human epithelial cells that is inhibited by growth factors EGF and IGF. Oncogene. 2003;22(30):4734-44.

22. Shimizu N, Yoshikawa N, Ito N, Maruyama T, Suzuki Y, Takeda S-i, et al. Crosstalk between glucocorticoid receptor and nutritional sensor mTOR in skeletal muscle. Cell metabolism. 2011;13:170-82.

23. Mammucari C, Milan G, Romanello V, Masiero E, Rudolf R, Del Piccolo P, et al. FoxO3 controls autophagy in skeletal muscle in vivo. Cell metabolism. 2007;6(6):458-71.

24. McLoughlin TJ, Smith SM, DeLong AD, Wang H, Unterman TG, Esser KA. FoxO1 induces apoptosis in skeletal myotubes in a DNA-binding-dependent manner. American journal of physiology Cell physiology. 2009;297(3):C548-55.

25. Wang H, Kubica N, Ellisen LW, Jefferson LS, Kimball SR. Dexamethasone represses signaling through the mammalian target of rapamycin in muscle cells by enhancing expression of REDD1. J Biol Chem. 2006;281(51):39128-34.

26. DeYoung MP, Horak P, Sofer A, Sgroi D, Ellisen LW. Hypoxia regulates TSC1/2-mTOR signaling and tumor suppression through REDD1-mediated 14-3-3 shuttling. Genes \& development. 2008;22:239-51.

27. $\mathrm{Xu} \mathrm{J,} \mathrm{Ji} \mathrm{J,} \mathrm{Yan} \mathrm{XH.} \mathrm{Cross-talk} \mathrm{between} \mathrm{AMPK} \mathrm{and} \mathrm{mTOR} \mathrm{in} \mathrm{regulating} \mathrm{energy} \mathrm{balance.} \mathrm{Criti-}$ cal reviews in food science and nutrition. 2012;52(5):373-81.

28. Brugarolas J, Lei K, Hurley RL, Manning BD, Reiling JH, Hafen E, et al. Regulation of mTOR function in response to hypoxia by REDD1 and the TSC1/TSC2 tumor suppressor complex. Genes \& development. 2004;18:2893-904. 
29. Vendelbo MH, Moller AB, Christensen B, Nellemann B, Clasen BF, Nair KS, et al. Fasting increases human skeletal muscle net phenylalanine release and this is associated with decreased mTOR signaling. PLoS One. 2014;9(7):e102031.

30. Shavlakadze T, Soffe Z, Anwari T, Cozens G, Grounds MD. Short-term feed deprivation rapidly induces the protein degradation pathway in skeletal muscles of young mice. J Nutr. 2013;143(4):403-9.

31. Hermans JJ, van Essen H, Struijker-Boudier HA, Johnson RM, Theeuwes F, Smits JF. Pharmacokinetic advantage of intrapericardially applied substances in the rat. The Journal of pharmacology and experimental therapeutics. 2002;301(2):672-8.

32. Mason SR, Ward LC, Reilly PE. Fluorimetric detection of serum corticosterone using highperformance liquid chromatography. Journal of chromatography. 1992;581(2):267-71.

33. Ma XM, Blenis J. Molecular mechanisms of mTOR-mediated translational control. Nat Rev Mol Cell Biol. 2009;10:307-18.

34. Miyazaki M, McCarthy JJ, Esser KA. Insulin like growth factor-1-induced phosphorylation and altered distribution of tuberous sclerosis complex (TSC)1/TSC2 in C2C12 myotubes. Febs J. 2010;277(9):2180-91.

35. Cai SL, Tee AR, Short JD, Bergeron JM, Kim J, Shen J, et al. Activity of TSC2 is inhibited by AKT-mediated phosphorylation and membrane partitioning. The Journal of cell biology. 2006;173(2):279-89.

36. Hayot M, Rodriguez J, Vernus B, Carnac G, Jean E, Allen D, et al. Myostatin up-regulation is associated with the skeletal muscle response to hypoxic stimuli. Molecular and cellular endocrinology. 2010.

37. Favier FB, Costes F, Defour A, Bonnefoy R, Lefai E, Baugé S, et al. Down-regulation of Akt/ mammalian target of rapamycin pathway in skeletal muscle is associated with increased REDD1 expression in response to chronic hypoxia. American journal of physiology Regulatory, integrative and comparative physiology. 2010:1659-66.

38. Bodine SC, Latres E, Baumhueter S, Lai VK, Nunez L, Clarke BA, et al. Identification of ubiquitin ligases required for skeletal muscle atrophy. Science. 2001;294(5547):1704-8.

39. Ogata T, Oishi Y, Higuchi M, Muraoka I. Fasting-related autophagic response in slowand fast-twitch skeletal muscle. Biochemical and biophysical research communications. 2010;394:136-40.

40. Wassner SJ, Orloff S, Holliday MA. Protein degradation in muscle: response to feeding and fasting in growing rats. The American journal of physiology. 1977;233(2):E119-23.

41. Dunn MA, Houtz SK, Hartsook EW. Effects of fasting on muscle protein turnover, the composition of weight loss, and energy balance of obese and nonobese Zucker rats. J Nutr. 1982;112(10):1862-75.

42. Asada M, Rauch A, Shimizu H, Maruyama H, Miyaki S, Shibamori M, et al. DNA bindingdependent glucocorticoid receptor activity promotes adipogenesis via Kruppel-like factor 15 gene expression. Laboratory investigation; a journal of technical methods and pathology. 2011;91(2):203-15. 
43. Zhao J, Brault JJ, Schild A, Goldberg AL. Coordinate activation of autophagy and the proteasome pathway by FoxO transcription factor. Autophagy. 2008;4:378-80.

44. Wochnik GM, Young JC, Schmidt U, Holsboer F, Hartl FU, Rein T. Inhibition of GR-mediated transcription by p23 requires interaction with Hsp90. FEBS letters. 2004;560(1-3):35-8.

45. Wochnik GM, Ruegg J, Abel GA, Schmidt U, Holsboer F, Rein T. FK506-binding proteins 51 and 52 differentially regulate dynein interaction and nuclear translocation of the glucocorticoid receptor in mammalian cells. J Biol Chem. 2005;280(6):4609-16.

46. Minet E, Mottet D, Michel G, Roland I, Raes M, Remacle J, et al. Hypoxia-induced activation of HIF-1: role of HIF-1alpha-Hsp90 interaction. FEBS letters. 1999;460(2):251-6.

47. Tarricone E, Scapin C, Vitadello M, Esposito F, Margonato V, Milano G, et al. Cellular distribution of Hsp70 expression in rat skeletal muscles. Effects of moderate exercise training and chronic hypoxia. Cell stress \& chaperones. 2008;13(4):483-95.

48. Magalhaes J, Ascensao A, Soares JM, Neuparth MJ, Ferreira R, Oliveira J, et al. Acute and severe hypobaric hypoxia-induced muscle oxidative stress in mice: the role of glutathione against oxidative damage. European journal of applied physiology. 2004;91(2-3):185-91.

49. Rupec RA, Poujol D, Kaltschmidt C, Messer G. Isolation of a hypoxia-induced cDNA with homology to the mammalian growth-related protein p23. Oncology research. 1998;10(2):69-74.

50. Cherel Y, Attaix D, Rosolowska-Huszcz D, Belkhou R, Robin JP, Arnal M, et al. Whole-body and tissue protein synthesis during brief and prolonged fasting in the rat. Clinical science. 1991;81(5):611-9.

51. Williamson DL, Li Z, Tuder RM, Feinstein E, Kimball SR, Dungan CM. Altered nutrient response of mTORC1 as a result of changes in REDD1 expression: effect of obesity vs. REDD1 deficiency. J Appl Physiol (1985). 2014;117(3):246-56.

52. Britto FA, Begue G, Rossano B, Docquier A, Vernus B, Sar C, et al. REDD1 deletion prevents dexamethasone-induced skeletal muscle atrophy. Am J Physiol Endocrinol Metab. 2014:ajpendo 002342014

53. Klein S, Sakurai Y, Romijn JA, Carroll RM. Progressive alterations in lipid and glucose metabolism during short-term fasting in young adult men. The American journal of physiology. 1993;265(5 Pt 1):E801-6.

54. Webber J, Macdonald IA. The cardiovascular, metabolic and hormonal changes accompanying acute starvation in men and women. The British journal of nutrition. 1994;71(3):437-47.

55. Gamboa JL, Garcia-Cazarin ML, Andrade FH. Chronic hypoxia increases insulin-stimulated glucose uptake in mouse soleus muscle. Am J Physiol Regul integr comp physiol. 2011;300:R85-91.

56. Hardie DG, Ross FA, Hawley SA. AMPK: a nutrient and energy sensor that maintains energy homeostasis. Nat Rev Mol Cell Biol. 2012;13(4):251-62.

57. Emerling BM, Weinberg F, Snyder C, Burgess Z, Mutlu GM, Viollet B, et al. Hypoxic activation of AMPK is dependent on mitochondrial ROS but independent of an increase in AMP/ATP ratio. Free Radic Biol Med. 2009;46(10):1386-91. 
58. Mungai PT, Waypa GB, Jairaman A, Prakriya M, Dokic D, Ball MK, et al. Hypoxia triggers AMPK activation through reactive oxygen species-mediated activation of calcium releaseactivated calcium channels. Mol Cell Biol. 2011;31(17):3531-45.

59. Mulligan JD, Gonzalez AA, Kumar R, Davis AJ, Saupe KW. Aging elevates basal adenosine monophosphate-activated protein kinase (AMPK) activity and eliminates hypoxic activation of AMPK in mouse liver. The journals of gerontology Series A, Biological sciences and medical sciences. 2005;60(1):21-7.

60. Mankouri J, Tedbury PR, Gretton S, Hughes ME, Griffin SD, Dallas ML, et al. Enhanced hepatitis $\mathrm{C}$ virus genome replication and lipid accumulation mediated by inhibition of AMPactivated protein kinase. Proc Natl Acad Sci U S A. 2010;107(25):11549-54.

61. Hawley SA, Boudeau J, Reid JL, Mustard KJ, Udd L, Makela TP, et al. Complexes between the LKB1 tumor suppressor, STRAD alpha/beta and MO25 alpha/beta are upstream kinases in the AMP-activated protein kinase cascade. Journal of biology. 2003;2(4):28.

62. Clanton TL. Hypoxia-induced reactive oxygen species formation in skeletal muscle. J Appl Physiol (1985). 2007;102(6):2379-88.

63. Li L, Chen Y, Gibson SB. Starvation-induced autophagy is regulated by mitochondrial reactive oxygen species leading to AMPK activation. Cellular signaling. 2013;25(1):50-65.

64. Pouw EM, Schols AM, van der Vusse GJ, Wouters EF. Elevated inosine monophosphate levels in resting muscle of patients with stable chronic obstructive pulmonary disease. American journal of respiratory and critical care medicine. 1998;157(2):453-7.

65. Calvert LD, Singh SJ, Greenhaff PL, Morgan MD, Steiner MC. The plasma ammonia response to cycle exercise in COPD. The European respiratory journal. 2008;31(4):751-8.

66. Sahlin K, Katz A. Hypoxaemia increases the accumulation of inosine monophosphate (IMP) in human skeletal muscle during submaximal exercise. Acta physiologica Scandinavica. 1989;136(2):199-203.

67. Sanchez AM, Candau RB, Csibi A, Pagano AF, Raibon A, Bernardi H. The role of AMP-activated protein kinase in the coordination of skeletal muscle turnover and energy homeostasis. American journal of physiology Cell physiology. 2012;303(5):C475-85.

68. Schneider A, Younis RH, Gutkind JS. Hypoxia-induced energy stress inhibits the mTOR pathway by activating an AMPK/REDD1 signaling axis in head and neck squamous cell carcinoma. Neoplasia. 2008;10(11):1295-302.

69. Nakashima K, Yakabe Y. AMPK activation stimulates myofibrillar protein degradation and expression of atrophy-related ubiquitin ligases by increasing FOXO transcription factors in C2C12 myotubes. Biosci Biotechnol Biochem. 2007;71(7):1650-6.

70. Zungu M, Schisler JC, Essop MF, McCudden C, Patterson C, Willis MS. Regulation of AMPK by the ubiquitin proteasome system. Am J Pathol. 2011;178(1):4-11.

71. Krawiec BJ, Nystrom GJ, Frost RA, Jefferson LS, Lang CH. AMP-activated protein kinase agonists increase mRNA content of the muscle-specific ubiquitin ligases MAFbx and MuRF1 in C2C12 cells. Am J Physiol Endocrinol Metab. 2007;292(6):E1555-67.

72. Egan D, Kim J, Shaw RJ, Guan KL. The autophagy initiating kinase ULK1 is regulated via opposing phosphorylation by AMPK and mTOR. Autophagy. 2011;7(6):643-4. 
73. Kim J, Kundu M, Viollet B, Guan KL. AMPK and mTOR regulate autophagy through direct phosphorylation of Ulk1. Nat Cell Biol. 2011;13(2):132-41.

74. Sanchez AM, Csibi A, Raibon A, Cornille K, Gay S, Bernardi H, et al. AMPK promotes skeletal muscle autophagy through activation of forkhead FoxO3a and interaction with Ulk1. J Cell Biochem. 2012;113(2):695-710. 
General discussion 


\section{Hypoxemia in COPD}

Muscle wasting is a common but often under-recognized extra-pulmonary feature of chronic obstructive pulmonary disease (COPD) that significantly increases disease burden. In addition to nutritional imbalance, systemic inflammation and progressive inactivity, hypoxemia has been implicated as a trigger for muscle atrophy in COPD patients. These patients may experience various degrees of oxygen desaturation, which could result in tissue hypoxia. This may occur with varying frequencies, for example during exercise (exertional desaturation) [1], during the night (nocturnal desaturation) [2], during disease exacerbations or chronically due to severely impaired lung function, such as in the case of chronic respiratory failure (CRF) [3]. Hypoxemic respiratory failure according to the classification by the Global Initiative for Chronic Obstructive Lung Disease (GOLD) is defined by an arterial partial pressure of $\mathrm{O} 2(\mathrm{PaO} 2)<8.0 \mathrm{kPa}(60 \mathrm{mmHg})$ with or without arterial partial pressure of $\mathrm{CO} 2(\mathrm{PaCO} 2)>6.7 \mathrm{kPa}(50 \mathrm{mmHg})$ when breathing ambient air [4]. The prevalence of hypoxemia among COPD patients remains uncertain [3]. In large general COPD populations, severe hypoxemia is relatively uncommon. In one study, only $2 \%$ of the 5993 participants received oxygen treatment [5], however, over $80 \%$ of the patients with advanced disease enrolled in the National Emphysema Treatment Trial were using some form of oxygen therapy [6]. According to the guidelines of the American Thoracic Society [7], patients receive oxygen treatment when the $\mathrm{PaO} 2$ level is $\leq 55 \mathrm{mmHg}$, or when hemoglobin oxygen saturation ( $\mathrm{SaO} 2)$ measured by a pulse oximeter $\leq 88$ $\%$ when breathing ambient air at rest. Chronic hypoxia leads to a poor prognosis and an increased mortality [8, 9], which is reduced by oxygen treatment [10-12]. During sleep, oxygen levels may drop even further, below $55 \mathrm{mmHg}$, which is then called nocturnal oxygen desaturation (NOD. This also occurs in patients with no desaturation at rest. A part of the COPD patients with $\mathrm{PaO} 2$ levels higher than $60 \mathrm{mmHg}$, showed rapid eye movement (REM) sleep related NOD [13-16]. Minami et al. showed that in a group of COPD patients with normal blood gases desaturation ( $\mathrm{SaO} 2<90 \%$ ) occurred more frequently during the night (day: $3.0 \%$ vs night: $7.2 \%$ of the measured time) [17]. In addition, oxygen levels may drop further during exacerbations to concentrations as low as PaO2: 27 - $45 \mathrm{mmHg}$ or SaO2: $35-80 \%$ [18]. Hypoxemia, either chronic or intermittent, is therefore an obvious feature of respiratory failure, but surprisingly its potential impact on the maintenance of muscle mass and oxidative capacity - which constitutes a second major aspect of muscle function - in COPD patients is rather unexplored. In an opinion paper incorporated in chapter 1 [19], we addressed the evidence that suggests that hypoxia may play a causal role in muscle atrophy and impaired oxidative capacity seen in patients with CRF. Existing experimental models were reviewed which illustrated that ambient hypoxia can result in muscle atrophy and 
metabolic impairments. The disrupted regulation of muscle mass and oxidative metabolism observed in these experimental models has also been shown in muscles of COPD patients, suggestive of but not conclusive for a causal role of hypoxia. We concluded that hypoxia affects skeletal muscle maintenance, but whether this concerns a direct effect of hypoxia on skeletal muscle remains to be confirmed. The underlying molecular mechanisms need to be investigated.

\section{Skeletal muscle tissue hypoxia}

To address the question how hypoxia affects skeletal muscle maintenance, we developed a mouse model with normobaric hypoxia (8\% oxygen) described in chapter 2 [20]. Acute hypoxia (4 days, 8\% oxygen) reduces the $\mathrm{PaO} 2$ values to $50 \mathrm{mmHg}$. SaO2 levels in these mice are much lower $(60 \%)$ than the $88 \%$ that are the threshold in patients to start oxygen treatment. After prolonged hypoxia (d21) in mice, the oxygen concentration drops to a level which is no longer comparable to what is found in clinically stable COPD patients but resembles that during an exacerbation or at high altitude exposure. We investigated the adaptations of protein turnover signaling in skeletal muscle in response to ambient hypoxia, therefore, the oxygen levels within the tissue itself, the so-called intracellular $\mathrm{O} 2$ partial pressure $(\mathrm{PiO} 2)$ had to be considered. Richardson et al [21], measured $\mathrm{PiO} 2$ in human skeletal muscle and found a value of $34 \pm 6 \mathrm{mmHg}$ when breathing normal air and of $23 \pm 6 \mathrm{mmHg}$ at $10 \%$ ambient oxygen. Reinke et al [22] measured $\mathrm{PiO} 2$ in mice and found a value of $52 \mathrm{mmHg}$ in normal air and of $17.7 \mathrm{mmHg}$ following exposure to $10 \%$ oxygen. Although $\mathrm{PiO} 2$ levels have not been described in muscle tissue of COPD patients, Vogiatzis et al [23] showed a reduced $\mathrm{StO} 2$ ( $\mathrm{StO} 2$ is the ratio of oxygenated hemoglobin to total hemoglobin) in quadriceps muscle from COPD patients at rest, suggesting lower oxygen levels in skeletal muscle. Using the hypoxia-sensitive probe pimonidazole [24], we tried to assess skeletal muscle oxygen levels in our mouse model. Pimonidazole is reductively activated in an oxygen-dependent manner and is covalently bound to thiol-containing proteins in hypoxic cells when oxygen levels drop below 10 $\mathrm{mmHg}$. When administered in vivo 1 hour prior to tissue collection, no pimonidazole staining was found in skeletal muscles from hypoxic mice exposed to $8 \%$ oxygen. We therefore conclude that the muscle tissue oxygen levels were above $10 \mathrm{mmHg}$. In chapter 2 [20], it was shown that expression of both carbonic anhydrase IX (CA-9) and BCL2/adenovirus E1B 19-kDa protein-interacting protein (BNIP3) is elevated under hypoxic conditions in C2C12 cells. In vivo, BNIP3 expression clearly indicated hypoxic conditions in the gastrocnemius muscle from 4 days onwards, but CA-9 expression was found only after longer exposure (21 days). 


\section{Hypoxia and dysphagia}

Hypoxia is known to reduce food-intake [25-27], and reduced food intake or fasting result in muscle atrophy [28, 29]. Moreover, Vermeeren et al showed that COPD patients hospitalized with an exacerbation, which is strongly associated with hypoxemic episodes [30] had a decreased (by 40\%) mean dietary intake, which slowly recovered to normal caloric intake after 4 days hospitalization [31]. A pair-fed group was included in our mouse experiments to assess the contribution of reduced food intake in hypoxia-induced muscle atrophy. We hypothesized that hypoxia-induced muscle atrophy and alterations in the regulation of muscle protein turnover include a hypoxia-specific component, in addition to the observed effects of reduced food intake in response to hypoxia. Hypoxia led to a decrease in gastrocnemius muscle weight and based on our different studies, up to $55 \%$ of the loss in gastrocnemius weight by hypoxia is accounted for by reduced food intake. Although only few studies on mice exposed to hypoxia report changes in muscle weights, a consistent finding is a reduction of muscle mass of up to $16 \%$ in response to hypoxia $[32,33]$. Surprisingly hardly any hypoxic study included a pair-fed group [34-39], whereas the importance of hypoxia-induced reduction of food intake was clearly illustrated in chapter 2 [20]. This is especially important in young animals since the reduced food intake may constitute a confounder by affecting growth [38]. In juvenile rats, the average muscle loss is up to $40 \%$ depending on severity of the hypoxic conditions, duration and age of the animals. In a study by Slot et al. [40] tibia length was decreased in juvenile (4-wk-old) mice by $4.5 \%$ after 3 weeks of hypoxia, suggesting growth retardation (Figure 1). Food restriction also decreased tibia length by $1.7 \%$, implicating that both inadequate food intake and hypoxia contribute to reduced muscle weight when compared to age-matched normoxic controls. Clearly, both the reduced food intake and a hypoxia-specific component contribute to hypoxia-induced muscle atrophy in a muscle of mixed fiber types, such as the gastrocnemius muscle.

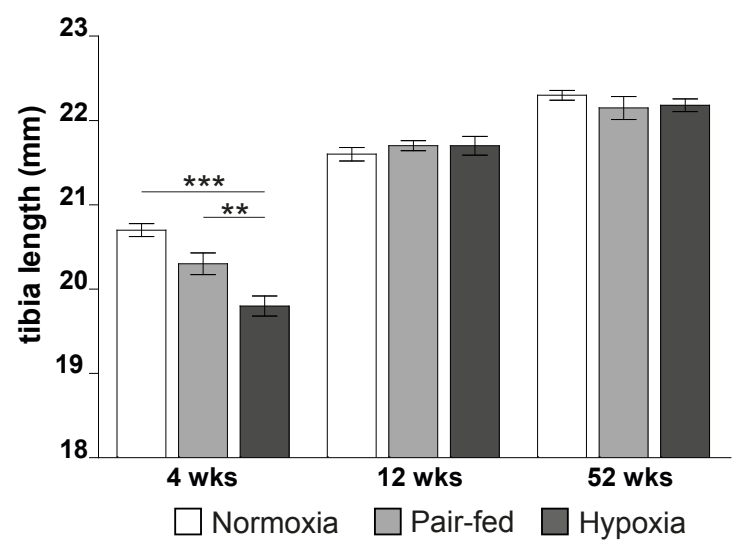

Figure 1. Tibia length of different aged mice in response to hypoxia. Tibia length was measured after 3 weeks of hypoxia or pair-feeding. Length was determined on intact hindlimbs, from bottom feet up to the top of the knee. Significant differences within each age-group are indicated by asterisks, ${ }^{* *}=\mathrm{P} \leq 0.01$ or ${ }^{* * *}=$ $P \leq 0.001$. $(n=6-9)$. 


\section{Hypoxia effects \& muscle type differences}

Different muscle fibers may respond differently to hypoxic conditions as shown for the oxidative soleus and the glycolytic extensor digitorum longus (EDL) muscles in chapter 3 [41]. Based on muscle mass and fiber cross section area (FCSA), hypoxia-induced muscle atrophy was more prominent in the EDL than in the soleus muscle. This was partially due to the reduced food intake. In addition to loss of muscle mass, peripheral muscles of COPD patients are often characterized by a shift from slow oxidative type I fibers towards fast glycolytic type II fibers. In adult mice (12-15 weeks), on the other hand, no fiber type shift was observed in either muscle type, despite the lowered FCSA of skeletal muscle under normobaric hypoxia. Slot et al [40] showed that such a shift is associated with a higher adaptive muscle plasticity in young (4 - 7 weeks) mice. The capillary density (CD) is lower in glycolytic muscles such as the EDL compared to the oxidative soleus muscle [42]. Furthermore, the FCSA per capillary contact (CC) is 1.5 times higher in the glycolytic muscle, suggesting that nutrients and oxygen have to travel larger distances in a glycolytic fiber compared to an oxidative fiber. The reduction of FCSA in the glycolytic muscle results in a reduced FCSA per capillary contact and thus enhances nutrient transport and gas exchange. In the oxidative muscle on the other hand, increase in CC rather than a decrease of the already low FCSA was observed. Accordingly, the FCSA per CC was reduced for both muscles, although by two distinct mechanisms. The largest differences were seen in the glycolytic muscle. The increased sensitivity of muscles composed of mainly type-II fibers to hypoxia suggests an increased susceptibility of skeletal muscle from COPD patients to hypoxia-induced atrophy as a consequence of the type I to type II shift observed in muscles of these patients.

\section{Inter-cellular effects of hypoxia}

Many studies address the muscle cell autonomous effects of hypoxia, i.e. the direct adaptations of skeletal muscle cells in vitro in response to hypoxia. In patients and animal studies, hypoxia may also affect other tissues and organs such as brain, intestines, kidneys or fat. Altered function of these tissues may evoke behavioral changes or the release of molecules, which affect skeletal muscle indirectly (Figure 2). Hypoxia-mediated reduction of food intake is possibly the result of increased leptin production by adipose tissue and reduced grehlin production in the brain and the stomach. Leptin is produced by adipose tissue in approximate proportion to fat stores [43]. Circulating leptin suppresses food intake and permits energy expenditure [44]. Furthermore, leptin regulates glucose and fatty acid metabolism [45] and may thereby affect skeletal muscle. Increased leptin levels in response to (intermittent) hypoxia have been reported [22, 46, 47] however, we found a decrease of leptin mRNA and reduced leptin plasma 
concentrations after 21 days of hypoxia [48]. This may reflect the large loss of fat tissue after 21 days of hypoxia ( $50 \%$ of normoxic control group). Furthermore it has been shown that hypoxia reduced ghrelin levels in juvenile but not adult rats $[49,50]$. In high altitude studies in humans, ghrelin was also decreased and the appetite inhibitor peptide YY was increased [51]. When hypoxia was combined with exercise, this resulted in reduced hunger and overall appetite, paralleled by reduced acetylated ghrelin levels [52]. Ghrelin has also been shown to directly target skeletal muscle where it induces AKT signaling, and prevents dexame thasone- and fasting-induced muscle atrophy [53, 54], suggesting that reduced ghrelin levels in response to hypoxia may be permissive for muscle atrophy. Hypoxia also increases inflammatory markers such as interleukin (IL)-6 in high altitude illness [55-57] and in hypoxic mouse studies [58-60]. Van den Borst et al. indeed [48] found increased plasma IL-6 levels after 21 days of hypoxia in that same model as described in chapter 2. Hypoxia increased serum levels of IL-1 $\beta$, IL-6, monocyte chemotactic protein 1 (MCP-1/CCL2), and tumor necrosis factor $\alpha$ (TNF $\alpha$ ), through HIF2 $\alpha$ in hypoxic adipose tissue of obese mice (reviewed by [61]). Inflammatory markers such as TNFa can trigger protein degradation through NF-kB signaling which results in muscle wasting [62-64].

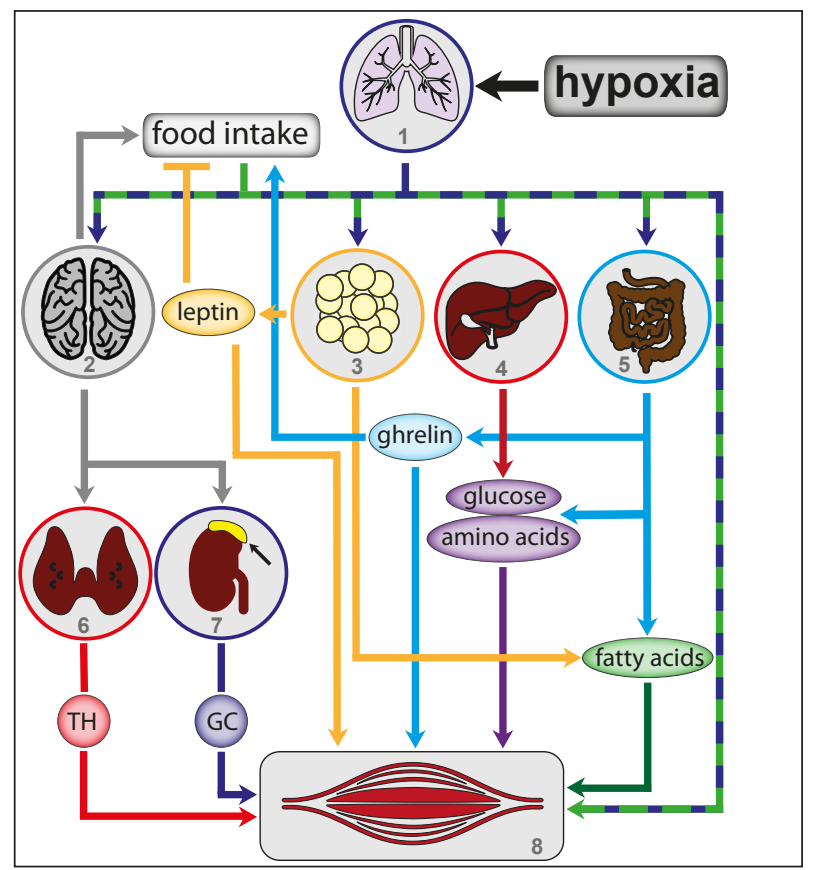

Figure 2. Overview of intercellular pathways discussed.

1: Lung, 2: Brain, 3: Adipose tissue (fat), 4: Liver, 5: Intestine, 6: Thyroid gland, 7: Adrenal cortex. TH: Thyroid hormone, GC: Glucocorticoid. 
Hypoxia has also been shown to increase corticotropin-releasing hormone $(\mathrm{CRH})$ mRNA and $\mathrm{CRH}$ release in the hypothalamus [65, 66]. Elevated levels of $\mathrm{CRH}$ stimulate the secretion of the adrenocorticotropic hormone (ACTH) from the anterior pituitary gland. ACTH stimulates glucocorticoid (GC) production by the adrenal cortex. GC production in response to hypoxia may be secondary to reduced food intake, as fasting increases circulating GC concentrations [28]. Indeed, we showed that hypoxia as well as the hypoxia-induced reduction in food intake result in a similar increase of plasma corticosterone (cc) (chapter 4). Hypoxemia has also been associated with elevated GC concentrations and GR signaling independent of malnutrition [67-71], which in turn have been associated with muscle atrophy [72]. We showed that GR deficiency prevents reduction of the gastrocnemius muscle mass and FCSA despite a reduced food intake (chapter 4). Under hypoxic conditions, loss of muscle mass and reduction of the FCSA was maintained despite GR deficiency. It has been shown that episodes of hypoxia evoke a profound disruption of gastrointestinal motility $[73,74]$ and it is well established that intestinal ischemia decreases absorption [75]. Accordingly, hypoxia also results in malabsorption [76], and as a consequence less macronutrients are available for the maintenance of muscle mass. Indeed, plasma glucose concentrations are reduced in mice exposed to 21 days of hypoxia (Figure 3 ). In addition, hypoglycemia results in reduced insulin release, with consequently decreased anabolic signaling in skeletal muscle. Moreover, apart from effects of hypoxia resulting in altered endocrine function and signals, hypoxia decreased food intake to such an extent, in our studies that plasma amino acid concentrations are probably affected [77]. This may directly contribute to a catabolic state in skeletal muscle considering its role as a latent amino acid reservoir, resulting in increased proteolysis and decreased protein synthesis. As the balance between protein degradation and synthesis is a major determinant of muscle mass, the intracellular regulation of these processes was investigated in detail in experiments described in this thesis.

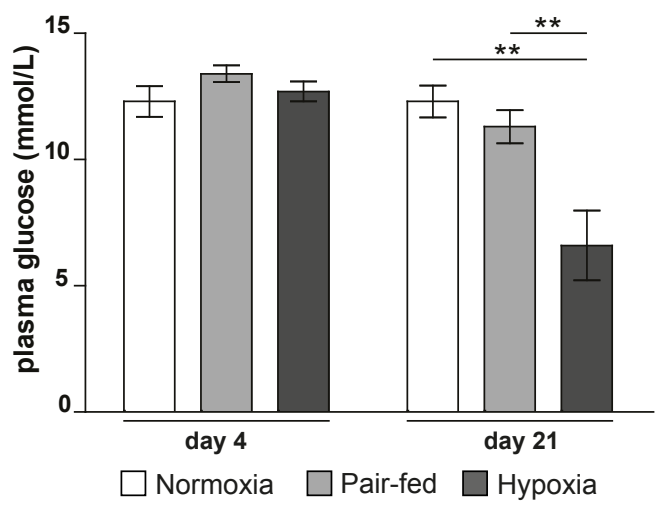

Figure 3. Plasma glucose concentrations in response to hypoxia. Significant differences within each time-group are indicated by asterisks, ${ }^{* *}=P \leq 0.01$. $(n=6-8)$. 


\section{Intra-cellular effects}

\section{Hypoxia-induced protein degradation regulation.}

Hypoxia clearly induced muscle atrophy, and induced the expression of genes involved in the regulation of protein degradation. Protein degradation via the ubiquitin proteasomal system (UPS) and autophagy-lysosomal pathway (ALP) are the main regulatory mechanism of protein degradation. We showed, that hypoxia and hypoxia-induced reduction of food intake induced both the UPS and ALP pathways [20, 41]. Glycolytic muscle of mice exposed to hypoxia showed a more pronounced UPS and ALP response compared to oxidative skeletal muscle [41]. While in oxidative muscle increased proteolytic signaling was mainly due to the reduced food intake, this was not the case for the proteolytic response of glycolytic muscle where additional hypoxia-dependent effects contributed. Hypoxia was also accompanied by increased signaling through the glucocorticoid receptor (GR), and it has been suggested that glycolytic muscle shows a higher sensitivity to glucocorticoids [78]. As muscle atrophy correlated with GR signaling and sensitivity, we hypothesized that hypoxia-induced muscle atrophy is GR dependent (chapter 4). GR deficiency blunted elevated ALP signaling in pairfed and hypoxic mice, whereas increased UPS signaling was reduced mainly in the pair-fed group (chapter 4). The sustained UPS signaling under hypoxic conditions in mGRKO mice suggested a secondary mechanism in addition to GR signaling. NF-KB signaling has been shown to induce protein degradation through the UPS [79]. Lin et al also showed that hypoxia-induced activation of HIF2 $\alpha$ in adipose tissue could activate NF-KB signaling in the heart [80]. Hypoxia or intestinal ischemia reperfusion also activate NF-KB in intestinal epithelial cells, which in turn increases the production of TNFa, a proinflammatory cytokine [81]. Additional interactions between hypoxia and inflammation are seen in the IKB kinase complex, a regulatory component of NF-KB [82] and in the regulation of HIF-1 $\alpha$ transcription by NF-KB during inflammation [83, 84]. Hypoxia amplifies the NF-KB pathway by increasing the expression and signaling of toll-like receptors [85]. This evidence for the induction of NF-KB signaling under hypoxic conditions raises the question to what extent muscle proteolysis in response to hypoxia depends on NF-kB. Stress-inducible transcription factors C/EBP or HIF1 $\alpha$, which respond to hypoxia, as well as fasting have been implicated in the control of Murf1 and Atrogin-1 expression. C/EBP's have been implicated in the transcriptional regulation of Atrogin-1 and Murf1 following glucocorticoid treatment [86], through interactions with GR [87] but also independently. C/EBP's interact with HIF1a, an interaction previously reported in human acute myeloid leukemia cells [88], and this interaction may contribute to GR-independent expression of Atrogin-1 and Murf1 under hypoxic conditions. Mothers against decapentaplegic homolog (Smad) transcription factors are part of TGF $\beta$ signaling [89]. Hypoxia has been 
shown to induce TGF $\beta$ signaling $[90,91]$ and HIF1 $\alpha$ participates in TGF $\beta$ induced transcription through physical protein-protein interaction [92]. There is also evidence that TGF $\beta$ is capable of increasing Atrogin-1 expression through SMAD3 [93]. Smad3 expression increases only in response to hypoxia and independent of GR (Figure 4) and may therefore contribute to expression of the atrogenes in response to hypoxia. TGF $\beta$ signaling is also involved in the response to myostatin. Myostatin is upregulated only in the glycolytic muscle in response to hypoxia and partially the result of reduced food intake. In the gastrocnemius muscle the increased expression of myostatin by reduced food intake and hypoxia was partially GR independent (data not shown) and may contribute to GR independent hypoxia-induced atrogene expression.

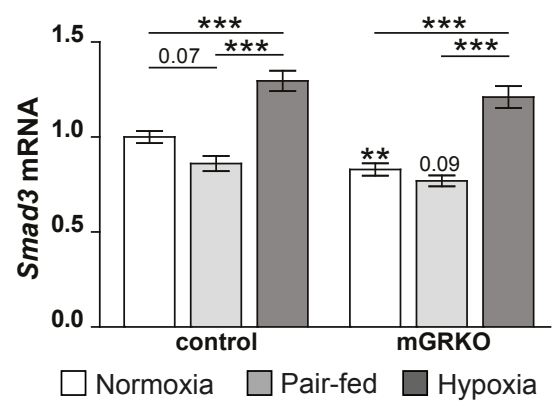

Figure 4. Smad3 mRNA expression levels in response to hypoxia. Significant differences between groups within control or mGRKO mice are indicated by asterisks on horizontal lines. Significant differences between control and $\mathrm{mGRKO}$ mice per condition are indicated by asterisks or trend above the column. ${ }^{* *}=\mathrm{P} \leq$ 0.01 or $^{* * *}=P \leq 0.001$. Trends $(0.05 \leq P \leq 0.1)$ are indicated by the specific $P$-value $(n=6-8)$

\section{Impaired regulation of protein synthesis under hypoxia.}

Reduced food intake, as a consequence of decreased availability of amino acids and decreased insulin concentrations, results in the attenuation of protein synthesis due to declined anabolic signals [94-96]. Accordingly, protein synthesis signaling is attenuated in skeletal muscle of pair-fed normoxic control mice [20]. In addition, most reports on cell-autonomous effects of hypoxia also indicate decreased protein synthesis [97, 98]. Therefore, the sustained mTORC1 signaling in glycolytic skeletal muscle under hypoxic conditions [20] despite the reduction of food intake is a major finding. The lack of mTORC1 inhibition by hypoxia is GR dependent (chapter 4) and mTORC1 activity is even maintained under hypoxia in response to fasting, which is a strong brake of protein synthesis (chapter 4)]. The deregulation of mTORC1 by hypoxia does not involve protein kinase $B$ (PKB/AKT) / tuberous sclerosis protein 2 (TSC2) and AKT / mammalian target of rapamycin (mTOR) signaling nor GR-dependent transcription of DNA-damageinducible transcript 4 protein (Ddit4/Redd1) or Krüppel-like factor 15 (KIf15) expression. GRs, when activated by circulating GCs, work through genomic or nongenomic mechanisms [99]. Typical genomic effects of GR are based on a DNA sequence called a glucocorticoid response element (GRE) and mostly mediate 
transactivation by recruiting coactivators and chromatin-remodeling complexes $[100,101]$. We identified glutamate-ammonia ligase (G/ul) and forkhead box $O 1$ (Foxo1) as direct genomic targets of GR, as GR deficiency completely abolished the transcriptional response of these genes to a reduction of food intake and hypoxia (chapter 4). Non-genomic actions include activation of signal transduction pathways such as the mitogen-activated protein kinase (MAPK) pathway by the membrane-bound or the cytoplasmic GR $[102,103]$. Another example of non-genomic GR actions involves suppression of protein synthesis through the inhibition of the phosphatidylinositol-4,5-bisphosphate 3-kinase (PI3K) / AKT / TSC1/2 / mTOR signaling pathway, in which GR activation prevents association of PI3K with insulin receptor substrate-1 (IRS-1), and subsequent downstream activation of AKT signaling [104]. mTORC1 activity in muscle of hypoxic mice was sustained even following $24 \mathrm{hrs}$ fasting (chapter 5 ). The mechanism by which hypoxia impairs inhibition of mTORC1 activity during fasting does not seem to rely on altered AKT/TSC2- nor AKT/mTOR signaling. In contrast, in response to fasting mTORC1 activity correlated to AMPK activity under normoxic and hypoxic conditions, suggesting that impaired AMPK activation under hypoxia is responsible for sustained mTORC1 activity. As the activity status and expression of TSC2 and REDD1, are altered accordingly, these molecules may constitute the regulatory circuit between AMPK and mTORC1 activity. Lack of AMPK activation by hypoxia has also been reported in older mice (24 months), although basal levels were already elevated by aging [105]. Hypoxia results in elevated levels of reactive oxygen species (ROS) in skeletal muscle [106] and it has been demonstrated that ROS production can block fasting-mediated AMPK activation [107]. In addition to ROS, inosine monophosphate (IMP) / adenosine monophosphate (AMP) / adenosine diphosphate (ADP) / adenosine triphosphate (ATP) concentrations may regulate AMPK activation. Elevated IMP but unchanged AMP, ADP and ATP levels have been demonstrated in COPD patients [108]. Increased AMP degradation to IMP and ammonia during exercise has also been shown in COPD patients [109]. Interestingly, hypoxemia (11\% ambient O2) resulted in increased IMP and stable AMP concentrations in human skeletal muscle during submaximal exercise, suggesting that hypoxia may interfere with AMP accumulation [110]. We have demonstrated in chapter 4 that GR is involved in the deregulation of mTORC1 during hypoxia, and we also implicate AMPK in the deregulation of mTORC1 during fasting under hypoxic conditions (chapter 4 and 5). Both proteins are involved in energy homeostasis. as the activated glucocorticoids receptor by glucocorticoids plays a central role in the regulation of energy metabolism by shifting it toward catabolism, whereas AMPK is the master regulator of energy homeostasis, sensing energy depletion and stimulating pathways of increasing fuel uptake and saving on peripheral supplies. Evidence suggesting crosstalk be- 
tween these metabolic regulators was provided by Nader et al. [111], who showed that AMPK through activation of its downstream substrate p38 MAPK, regulated GR-induced transcriptional activity by phosphorylating its serine residue at amino acid 211. AMPK-mediated phosphorylation of serine 211 of the human GR resulted in altered accumulation of cofactors to ligand-activated GR bound on GREs of glucocorticoid-responsive genes, possibly by preferential attraction/dissociation of phosphorylation site-specific cofactors [111]. Bouazza et al. [112] confirmed that p38 MAPK can phosphorylate GR at Ser211 thereby modifying GR transcriptional activity. We investigated phosphorylation of GR at Ser211 (Figure 5) in response to fasting under hypoxic conditions. Interestingly, GR phosphorylation is upregulated by fasting in the pair-fed group only but not under hypoxic conditions, similar to AMPK activity. This not only suggests that hypoxia may alter the transcriptional response of GR, but also indicates the intricate cross-talk between GR and AMPK as major metabolic signaling pathways, and emphasizes their candidature for further research in understanding the molecular basis of impaired protein synthesis regulation in skeletal muscle in response to hypoxia.
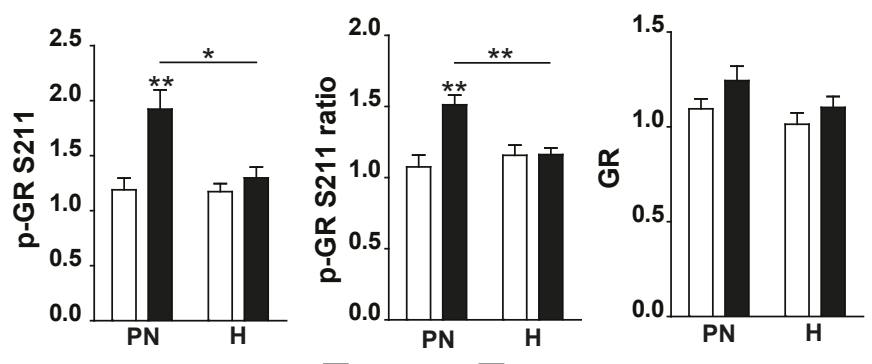

$\square$ control $\square 24$ hrs fasted

Figure 5. GR Ser211 phosphorylation in response to fasting under hypoxic conditions. Data are normalized to the basal expression of the normoxic group at day 0 . Significant differences between groups are indicated by asterisks, with ${ }^{*}=P<0.05,{ }^{* *}=P \leq 0.01$. Trends $(0.05 \leq P \leq 0.1)$ are indicated by the specific $P$-value $(n=6-8)$.

\section{Cellular and whole body consequences of impaired coordina- tion of muscle protein synthesis and degradation}

Coordinated changes in muscle protein synthesis and degradation determine alterations in muscle mass. Protein synthesis is suppressed and degradation increased in conditions such as fasting, glucocorticoids and hypoxia [72, 79, 113-115] ultimately resulting in muscle mass loss. Accordingly, under normoxic conditions, muscle atrophy in response to reduced food intake in the pair-fed groups or during fasting, is accompanied by attenuated protein synthesis signaling while regulatory steps in protein degradation suggest increased proteolysis. Under hypoxic conditions, our study suggests the opposite, as no inhibition of protein synthesis regulation was observed. Moreover, this was accompanied by 
elevated protein degradation signaling. To further address the consequences of the impaired regulation of protein synthesis signaling by hypoxia, subsequent studies should include measurements of protein synthesis rate. Normal protein synthesis regulation and elevated protein degradation suggest a hypermetabolic state. Evidence of a hypermetabolic state besides elevated protein turnover is seen in the decreased muscle mass and fat tissue weights in response to hypoxia. Furthermore, amino acids released from the degrading skeletal muscle will be used by other tissues to synthesize proteins, or will be converted into glucose by the liver (gluconeogenesis) if glycogen levels are depleted. We observed a reduced release of amino acids under hypoxic conditions in response to fasting (data not shown). This would implicate that muscle is no longer functioning as a store of amino acids and instead re-uses the amino acids freed by protein degradation. This could affect hepatic gluconeogenesis activity resulting in decreased plasma glucose levels (Figure 3). Despite sustained protein synthesis signaling under hypoxia, muscle mass loss is equal or even more pronounced under hypoxia, when compared to the pair-fed group. This is suggestive of increased protein turnover. Interestingly, in patients with COPD and muscle atrophy, elevated protein turnover signaling in skeletal muscle has also been reported [116, 117]. $26 \%$ Percent of the COPD patients studied by Creutzberg et al. [118] displayed a higher resting energy expenditure (REE) compared to the REE predicted on the basis of their fat-free mass (REEFFM). Hypermetabolism is one of the major symptoms of an overactive thyroid (hyperthyroidism). Hypothyroidism and hyperthryroidism are features of COPD patients as illustrated by Terzano et al. [125]. Elevated thyroid hormone $(\mathrm{TH})$ release was also seen in studies with high altitude exposure [119]. Hyperthyroidism furthermore leads to a shift of type I to type II fibers [120, 121]. It has also been suggested that a hypermetabolic state within skeletal muscle results from mitochondrial dysfunction [122], which can be triggered by hypoxia [33, 123]. In advanced COPD, mitochondrial dysfunction is likely present as skeletal muscles are characterized by decreased mitochondrial density and biogenesis, impaired activity and coupling of mitochondrial respiratory chain complexes, increased mitochondrial production of reactive oxygen species and possibly increased mitochondrial apoptosis [124]. Many factors besides hypoxia may contribute to mitochondrial dysfunction such as a sedentary lifestyle, hypercapnia, tobacco smoking, corticosteroid therapy and inflammation, and all these factors have also been implied in muscle atrophy. Therefore, future studies should address the potential interactions between mitochondrial dysfunction and loss of muscle mass. 


\section{Conclusion and future prospective}

This thesis shows that systemic hypoxia induces hypoxia-induced muscle atrophy. Glycolytic muscles are more sensitive to hypoxia-induced muscle atrophy. Hypoxia affects the regulation of protein degradation independent of GR signaling, but the exact mechanism remains to be explored. Hypoxia further impairs the inhibition of mTORC1 activity in response to reduced food-intake. This involves GR and AMPK signaling but the exact mechanism is also not fully understood. It is further speculated that normal regulation of protein synthesis regulation and increased stimulation of genes involved in protein degradation by hypoxia are features of a hypermetabolic state. Future studies should address the effects of hypoxia on protein synthesis and degradation rate directly to establish whether protein turnover is indeed increased. The novel finding that GR positively regulates mTORC1 activity should be explored as it is unknown how this affects the mTORC1 complex. It would also be interesting to explore the regulation of AMPK under hypoxic condition as it's response to fasting is altered under hypoxic conditions. 


\section{References}

1. van Gestel AJ, Clarenbach CF, Stowhas AC, Teschler S, Russi EW, Teschler H, et al. Prevalence and prediction of exercise-induced oxygen desaturation in patients with chronic obstructive pulmonary disease. Respiration; international review of thoracic diseases. 2012;84(5):353-9.

2. Lacasse Y, Series F, Vujovic-Zotovic N, Goldstein R, Bourbeau J, Lecours R, et al. Evaluating nocturnal oxygen desaturation in COPD--revised. Respiratory medicine. 2011;105(9):1331-7.

3. Kent BD, Mitchell PD, McNicholas WT. Hypoxemia in patients with COPD: cause, effects, and disease progression. International journal of chronic obstructive pulmonary disease. 2011;6:199-208.

4. Global Initiative for Chronic Obstructive Lung Disease (GOLD). Global strategy for the diagnosis, management and prevention of chronic pulmonary disease. 2015(1 Feb).

5. Tashkin DP, Celli B, Senn S, Burkhart D, Kesten S, Menjoge S, et al. A 4-year trial of tiotropium in chronic obstructive pulmonary disease. The New England journal of medicine. 2008;359(15):1543-54.

6. Martinez FJ, Foster G, Curtis JL, Criner G, Weinmann G, Fishman A, et al. Predictors of mortality in patients with emphysema and severe airflow obstruction. American journal of respiratory and critical care medicine. 2006;173(12):1326-34.

7. Celli BR, MacNee W. Standards for the diagnosis and treatment of patients with COPD: a summary of the ATS/ERS position paper. The European respiratory journal. 2004;23(6):93246.

8. Boushy SF, Thompson HK, Jr., North LB, Beale AR, Snow TR. Prognosis in chronic obstructive pulmonary disease. The American review of respiratory disease. 1973;108(6):1373-83.

9. Jones NL, Burrows B, Fletcher CM. Serial studies of 100 patients with chronic airway obstruction in London and Chicago. Thorax. 1967;22(4):327-35.

10. Neff TA, Petty TL. Long-term continuous oxygen therapy in chronic airway obstruction. Mortality in relationship to cor pulmonale, hypoxia, and hypercapnia. Annals of internal medicine. 1970;72(5):621-6.

11. Group NOTT. Continuous or nocturnal oxygen therapy in hypoxemic chronic obstructive lung disease: a clinical trial. Nocturnal Oxygen Therapy Trial Group. Annals of internal medicine. 1980;93(3):391-8.

12. Party MRCW. Long term domiciliary oxygen therapy in chronic hypoxic cor pulmonale complicating chronic bronchitis and emphysema. Report of the Medical Research Council Working Party. Lancet. 1981;1(8222):681-6.

13. Fletcher EC, Miller J, Divine GW, Fletcher JG, Miller T. Nocturnal oxyhemoglobin desaturation in COPD patients with arterial oxygen tensions above $60 \mathrm{~mm} \mathrm{Hg}$. Chest. 1987;92(4):604-8.

14. Mulloy E, Fitzpatrick M, Bourke S, O'Regan A, McNicholas WT. Oxygen desaturation during sleep and exercise in patients with severe chronic obstructive pulmonary disease. Respiratory medicine. 1995;89(3):193-8. 
15. Koo KW, Sax DS, Snider GL. Arterial blood gases and pH during sleep in chronic obstructive pulmonary disease. The American journal of medicine. 1975;58(5):663-70.

16. De Angelis G, Sposato B, Mazzei L, Giocondi F, Sbrocca A, Propati A, et al. Predictive indexes of nocturnal desaturation in COPD patients not treated with long term oxygen therapy. European review for medical and pharmacological sciences. 2001;5(5-6):173-9.

17. Minami S, Yamamoto S, Ogata Y, Nakatani T, Takeuchi Y, Hamaguchi M, et al. Ambulatory pulse oximetry monitoring in Japanese COPD outpatients not receiving oxygen therapy. Multidisciplinary respiratory medicine. 2014;9(1):24.

18. Henrion J, Minette P, Colin L, Schapira M, Delannoy A, Heller FR. Hypoxic hepatitis caused by acute exacerbation of chronic respiratory failure: a case-controlled, hemodynamic study of 17 consecutive cases. Hepatology. 1999;29(2):427-33.

19. de Theije C, Costes F, Langen RC, Pison C, Gosker HR. Hypoxia and muscle maintenance regulation: implications for chronic respiratory disease. Curr Opin Clin Nutr Metab Care. 2011;14(6):548-53.

20. de Theije CC, Langen RC, Lamers WH, Schols AM, Koehler SE. Distinct responses of protein turnover-regulatory pathways in hypoxia- and semi-starvation-induced muscle atrophy. Am J Physiol Lung Cell Mol Physiol. 2013.

21. Richardson RS, Duteil S, Wary C, Wray DW, Hoff J, Carlier PG. Human skeletal muscle intracellular oxygenation: the impact of ambient oxygen availability. The Journal of physiology. 2006;571(Pt 2):415-24.

22. Reinke C, Bevans-Fonti S, Drager LF, Shin MK, Polotsky VY. Effects of different acute hypoxic regimens on tissue oxygen profiles and metabolic outcomes. J Appl Physiol (1985). 2011;111(3):881-90.

23. Vogiatzis I, Athanasopoulos D, Stratakos G, Garagouni C, Koutsoukou A, Boushel R, et al. Exercise-induced skeletal muscle deoxygenation in O-supplemented COPD patients. Scandinavian journal of medicine \& science in sports. 2009;19(3):364-72.

24. Varia MA, Calkins-Adams DP, Rinker LH, Kennedy AS, Novotny DB, Fowler WC, Jr., et al. Pimonidazole: a novel hypoxia marker for complementary study of tumor hypoxia and cell proliferation in cervical carcinoma. Gynecologic oncology. 1998;71(2):270-7.

25. Kozak W, Wrotek S. Hypoxia-induced sickness beha viour. Pharmacology. 2006:35-50.

26. Chen XQ, Dong J, Niu CY, Fan JM, Du JZ. Effects of hypoxia on glucose, insulin, glucagon, and modulation by corticotropin-releasing factor receptor type 1 in the rat. Endocrinology. 2007;148(7):3271-8.

27. Westerterp-plantenga MS, Westerterp KR, Rubbens M, Verwegen RT, Richelet J-p, Gardette B, et al. Appetite at " high altitude " [ Operation Everest III ( Comex- ' 97 )]: a simulated ascent of Mount Everest effects of caloric restriction J Appl Physiol (1985). 2011:391-9.

28. Medina R, Wing SS, Goldberg AL. Increase in levels of polyubiquitin and proteasome mRNA in skeletal muscle during starvation and denervation atrophy. Biochem J. 1995;307 ( Pt 3):631-7. 
29. Wing SS, Goldberg AL. Glucocorticoids activate the ATP-ubiquitin-dependent proteolytic system in skeletal muscle during fasting. The American journal of physiology. 1993;264(4 Pt 1):E668-76.

30. Vermeeren MA, Wouters EF, Geraerts-Keeris AJ, Schols AM. Nutritional support in patients with chronic obstructive pulmonary disease during hospitalization for an acute exacerbation; a randomized controlled feasibility trial. Clinical nutrition. 2004;23(5):1184-92.

31. Vermeeren MA, Schols AM, Wouters EF. Effects of an acute exacerbation on nutritional and metabolic profile of patients with COPD. The European respiratory journal. 1997;10(10):22649.

32. Hauerslev S, Vissing J, Krag TO. Muscle atrophy reversed by growth factor activation of satellite cells in a mouse muscle atrophy model. PLoS One. 2014;9(6):e100594.

33. Magalhaes J, Ascensao A, Soares JM, Ferreira R, Neuparth MJ, Marques F, et al. Acute and severe hypobaric hypoxia increases oxidative stress and impairs mitochondrial function in mouse skeletal muscle. J Appl Physiol (1985). 2005;99(4):1247-53.

34. Hoppeler H, Kleinert E, Schlegel C, Claassen H, Howald H, Kayar SR, et al. Morphological adaptations of human skeletal muscle to chronic hypoxia. Int J Sports Med. 1990;11 Suppl 1:S3-9.

35. Gamboa JL, Garcia-Cazarin ML, Andrade FH. Chronic hypoxia increases insulin-stimulated glucose uptake in mouse soleus muscle. Am J Physiol Regul integr comp physiol. 2011;300:R85-91.

36. Favier FB, Costes F, Defour A, Bonnefoy R, Lefai E, Baugé S, et al. Down-regulation of Akt/ mammalian target of rapamycin pathway in skeletal muscle is associated with increased REDD1 expression in response to chronic hypoxia. American journal of physiology Regulatory, integrative and comparative physiology. 2010:1659-66.

37. Itoh K, Moritani T, Ishida K, Hirofuji C, Taguchi S, Itoh M. Hypoxia-induced fibre type transformation in rat hindlimb muscles. Histochemical and electro-mechanical changes. Eur J Appl Physiol Occup Physiol. 1990;60(5):331-6.

38. Abdelmalki A, Fimbel S, Mayet-Sornay MH, Sempore B, Favier R. Aerobic capacity and skeletal muscle properties of normoxic and hypoxic rats in response to training. Pflugers Archiv : European journal of physiology. 1996;431(5):671-9.

39. Tanaka M, Mizuta K, Koba F, Ohira Y, Kobayashi T, Honda Y. Effects of exposure to hypobaric-hypoxia on body weight, muscular and hematological characteristics, and work performance in rats. Jpn J Physiol. 1997;47(1):51-7.

40. Slot IG, Schols AM, de Theije CC, Snepvangers FJ, Gosker HR. Alterations in Skeletal Muscle Oxidative Phenotype in Mice Exposed to Three Weeks of Normobaric Hypoxia. Journal of cellular physiology. 2015.

41. de Theije CC, Langen RC, Lamers WH, Gosker HR, Schols AM, Koehler SE. Differential sensitivity of oxidative and glycolytic muscles to hypoxia-induced muscle atrophy. J Appl Physiol (1985). 2014:jap 006242014. 
42. Murakami S, Fujino H, Takeda I, Momota R, Kumagishi K, Ohtsuka A. Comparison of capillary architecture between slow and fast muscles in rats using a confocal laser scanning microscope. Acta medica Okayama. 2010;64:11-8.

43. Robertson SA, Leinninger GM, Myers MG, Jr. Molecular and neural mediators of leptin action. Physiol Behav. 2008;94(5):637-42.

44. Kelesidis T, Kelesidis I, Chou S, Mantzoros CS. Narrative review: the role of leptin in human physiology: emerging clinical applications. Annals of internal medicine. 2010;152(2):93-100.

45. Ceddia RB. Direct metabolic regulation in skeletal muscle and fat tissue by leptin: implications for glucose and fatty acids homeostasis. Int J Obes (Lond). 2005;29(10):1175-83.

46. Qin L, Song Z, Wen SL, Jing R, Li C, Xiang Y, et al. Effect of intermittent hypoxia on leptin and leptin receptor expression in obesity mice. Sheng Li Xue Bao. 2007;59(3):351-6.

47. Wang B, Wood IS, Trayhurn P. Hypoxia induces leptin gene expression and secretion in human preadipocytes: differential effects of hypoxia on adipokine expression by preadipocytes. The Journal of endocrinology. 2008;198(1):127-34.

48. van den Borst B, Schols AM, de Theije C, Boots AW, Kohler SE, Goossens GH, et al. Characterization of the inflammatory and metabolic profile of adipose tissue in a mouse model of chronic hypoxia. J Appl Physiol (1985). 2013;114(11):1619-28.

49. Chaiban JT, Bitar FF, Azar ST. Effect of chronic hypoxia on leptin, insulin, adiponectin, and ghrelin. Metabolism: clinical and experimental. 2008;57(8):1019-22.

50. Raff $H$. Total and active ghrelin in developing rats during hypoxia. Endocrine. 2003;21(2):15961.

51. Wasse LK, Sunderland C, King JA, Batterham RL, Stensel DJ. Influence of rest and exercise at a simulated altitude of 4,000 $\mathrm{m}$ on appetite, energy intake, and plasma concentrations of acylated ghrelin and peptide YY. J Appl Physiol (1985). 2012;112(4):552-9.

52. Bailey DP, Smith LR, Chrismas BC, Taylor L, Stensel DJ, Deighton K, et al. Appetite and gut hormone responses to moderate-intensity continuous exercise versus high-intensity interval exercise, in normoxic and hypoxic conditions. Appetite. 2015;89:237-45.

53. Barazzoni R, Zanetti M, Cattin MR, Visintin L, Vinci P, Cattin L, et al. Ghrelin enhances in vivo skeletal muscle but not liver AKT signaling in rats. Obesity (Silver Spring). 2007;15(11):261423.

54. Porporato PE, Filigheddu N, Reano S, Ferrara M, Angelino E, Gnocchi VF, et al. Acylated and unacylated ghrelin impair skeletal muscle atrophy in mice. The Journal of clinical investigation. 2013;123(2):611-22.

55. Semenza GL. Life with oxygen. Science. 2007;318(5847):62-4.

56. Hackett PH, Roach RC. High-altitude illness. The New England journal of medicine. 2001;345(2):107-14.

57. Grocott MP, Martin DS, Levett DZ, McMorrow R, Windsor J, Montgomery HE. Arterial blood gases and oxygen content in climbers on Mount Everest. The New England journal of medicine. 2009;360(2):140-9. 
58. Rosenberger P, Schwab JM, Mirakaj V, Masekowsky E, Mager A, Morote-Garcia JC, et al. Hypoxia-inducible factor-dependent induction of netrin-1 dampens inflammation caused by hypoxia. Nature immunology. 2009;10(2):195-202.

59. Eckle T, Faigle M, Grenz A, Laucher S, Thompson LF, Eltzschig HK. A2B adenosine receptor dampens hypoxia-induced vascular leak. Blood. 2008;111(4):2024-35.

60. Eltzschig HK, Carmeliet P. Hypoxia and inflammation. The New England journal of medicine. 2011;364(7):656-65.

61. Lin Q, Yun Z. The Hypoxia-Inducible Factor Pathway in Adipocytes: The Role of HIF-2 in Adipose Inflammation and Hypertrophic Cardiomyopathy. Frontiers in endocrinology. 2015;6:39.

62. Reid MB, Li YP. Tumor necrosis factor-alpha and muscle wasting: a cellular perspective. Respir Res. 2001;2(5):269-72.

63. Strassmann G, Fong M, Kenney JS, Jacob CO. Evidence for the involvement of interleukin 6 in experimental cancer cachexia. The Journal of clinical investigation. 1992;89(5):1681-4.

64. Doyle A, Zhang G, Abdel Fattah EA, Eissa NT, Li YP. Toll-like receptor 4 mediates lipopolysaccharide-induced muscle catabolism via coordinate activation of ubiquitin-proteasome and autophagy-lysosome pathways. FASEB journal : official publication of the Federation of American Societies for Experimental Biology. 2011;25(1):99-110.

65. Feng XW, Kang J, Wang ZF, Wang W, Yu RJ. [The effect of chronic intermittent hypoxia to hypothalamus-pituitary-adrenal axis and growth hormone level in rats during sleep]. Zhongguo ying yong sheng li xue za zhi = Zhongguo yingyong shenglixue zazhi $=$ Chinese journal of applied physiology. 2005;21(4):414-7.

66. Chen XQ, Du JZ, Wang YS. Regulation of hypoxia-induced release of corticotropin-releasing factor in the rat hypothalamus by norepinephrine. Regulatory peptides. 2004;119(3):221-8.

67. Gardner DS, Fletcher AJ, Fowden AL, Giussani DA. Plasma adrenocorticotropin and cortisol concentrations during acute hypoxemia after a reversible period of adverse intrauterine conditions in the ovine fetus during late gestation. Endocrinology. 2001;142(2):589-98.

68. Kodama T, Shimizu N, Yoshikawa N, Makino Y, Ouchida R, Okamoto K, et al. Role of the glucocorticoid receptor for regulation of hypoxia-dependent gene expression. J Biol Chem. 2003;278(35):33384-91.

69. Moncloa F, Donayre J, Sobrevilla LA, Guerra-Garcia R. Endocrine studies at high altitude. II. Adrenal cortical function in sea level natives exposed to high altitudes (4300 metersfor two weeks. The Journal of clinical endocrinology and metabolism. 1965;25(12):1640-2.

70. Larsen JJ, Hansen JM, Olsen NV, Galbo H, Dela F. The effect of altitude hypoxia on glucose homeostasis in men. The Journal of physiology. 1997;504 ( Pt 1):241-9.

71. Leonard MO, Godson C, Brady HR, Taylor CT. Potentiation of glucocorticoid activity in hypoxia through induction of the glucocorticoid receptor. Journal of immunology. 2005;174(4):22507.

72. Schakman O, Kalista S, Barbe C, Loumaye A, Thissen JP. Glucocorticoid-induced skeletal muscle atrophy. The international journal of biochemistry \& cell biology. 2013;45(10):2163-72. 
73. Wood JG, Yan ZY, Zhang Q, Cheung LY. Ischemia-reperfusion increases gastric motility and endothelin-1-induced vasoconstriction. The American journal of physiology. 1995;269(4 Pt 1):G524-31.

74. Bielefeldt K, Conklin JL. Intestinal motility during hypoxia and reoxygenation in vitro. Digestive diseases and sciences. 1997;42(5):878-84.

75. Robinson JW, Mirkovitch V, Winistorfer B, Saegesser F. Response of the intestinal mucosa to ischaemia. Gut. 1981;22(6):512-27.

76. Lifshitz F, Wapnir RA, Teichberg S. Alterations in jejunal transport and ( $\mathrm{Na+-K+)-ATPase} \mathrm{in} \mathrm{an}$ experimental model of hypoxia in rats. Proceedings of the Society for Experimental Biology and Medicine Society for Experimental Biology and Medicine. 1986;181(1):87-97.

77. Wu C. Metabolism of free amino acids in fasted and zein-fed rats. J Biol Chem. 1954;207(2):775-86.

78. Livingstone I, Johnson MA, Mastaglia FL. Effects of dexamethasone on fibre subtypes in rat muscle. Neuropathol Appl Neurobiol. 1981;7(5):381-98.

79. Glass DJ. Skeletal muscle hypertrophy and atrophy signaling pathways. The international journal of biochemistry \& cell biology. 2005;37(10):1974-84.

80. Lin Q, Huang Y, Booth CJ, Haase VH, Johnson RS, Celeste Simon M, et al. Activation of hypoxia-inducible factor-2 in adipocytes results in pathological cardiac hypertrophy. Journal of the American Heart Association. 2013;2(6):e000548.

81. Chen LW, Egan L, Li ZW, Greten FR, Kagnoff MF, Karin M. The two faces of IKK and NFkappaB inhibition: prevention of systemic inflammation but increased local injury following intestinal ischemia-reperfusion. Nature medicine. 2003;9(5):575-81.

82. Cummins EP, Berra E, Comerford KM, Ginouves A, Fitzgerald KT, Seeballuck F, et al. Prolyl hydroxylase-1 negatively regulates IkappaB kinase-beta, giving insight into hypoxia-induced NFkappaB activity. Proc Natl Acad Sci U S A. 2006;103(48):18154-9.

83. Bonello S, Zahringer C, BelAiba RS, Djordjevic T, Hess J, Michiels C, et al. Reactive oxygen species activate the HIF-1alpha promoter via a functional NFkappaB site. Arteriosclerosis, thrombosis, and vascular biology. 2007;27(4):755-61.

84. Rius J, Guma M, Schachtrup C, Akassoglou K, Zinkernagel AS, Nizet V, et al. NF-kappaB links innate immunity to the hypoxic response through transcriptional regulation of HIF-1alpha. Nature. 2008;453(7196):807-11.

85. Kuhlicke J, Frick JS, Morote-Garcia JC, Rosenberger P, Eltzschig HK. Hypoxia inducible factor (HIF)-1 coordinates induction of Toll-like receptors TLR2 and TLR6 during hypoxia. PLoS One. 2007;2(12):e1364.

86. Leis H, Page A, Ramirez A, Bravo A, Segrelles C, Paramio J, et al. Glucocorticoid Receptor Counteracts Tumorigenic Activity of Akt in Skin through Interference with the Phosphatidylinositol 3-Kinase Signaling Pathway. Molecular endocrinology. 2004;18(2):303-11.

87. Rudiger JJ, Roth M, Bihl MP, Cornelius BC, Johnson M, Ziesche R, et al. Interaction of C/ EBPalpha and the glucocorticoid receptor in vivo and in nontransformed human cells. FASEB journal : official publication of the Federation of American Societies for Experimental Biology. 2002;16(2):177-84. 
88. Jiang Y, Xue ZH, Shen WZ, Du KM, Yan H, Yu Y, et al. Desferrioxamine induces leukemic cell differentiation potentially by hypoxia-inducible factor-1 alpha that augments transcriptional activity of CCAAT/enhancer-binding protein-alpha. Leukemia. 2005;19(7):1239-47.

89. Massague J, Seoane J, Wotton D. Smad transcription factors. Genes \& development. 2005;19(23):2783-810.

90. Falanga V, Qian SW, Danielpour D, Katz MH, Roberts AB, Sporn MB. Hypoxia upregulates the synthesis of TGF-beta 1 by human dermal fibroblasts. The Journal of investigative dermatology. 1991;97(4):634-7.

91. Suzuki A, Kusakai G, Shimojo Y, Chen J, Ogura T, Kobayashi M, et al. Involvement of transforming growth factor-beta 1 signaling in hypoxia-induced tolerance to glucose starvation. J Biol Chem. 2005;280(36):31557-63.

92. Sanchez-Elsner T, Botella LM, Velasco B, Corbi A, Attisano L, Bernabeu C. Synergistic cooperation between hypoxia and transforming growth factor-beta pathways on human vascular endothelial growth factor gene expression. J Biol Chem. 2001;276(42):38527-35.

93. Goodman CA, McNally RM, Hoffmann FM, Hornberger TA. Smad3 induces atrogin-1, inhibits mTOR and protein synthesis, and promotes muscle atrophy in vivo. Molecular endocrinology. 2013;27(11):1946-57.

94. Cherel Y, Attaix D, Rosolowska-Huszcz D, Belkhou R, Robin JP, Arnal M, et al. Whole-body and tissue protein synthesis during brief and prolonged fasting in the rat. Clinical science. 1991;81(5):611-9.

95. Dunn MA, Houtz SK, Hartsook EW. Effects of fasting on muscle protein turnover, the composition of weight loss, and energy balance of obese and nonobese Zucker rats. J Nutr. 1982;112(10):1862-75.

96. Vendelbo MH, Moller AB, Christensen B, Nellemann B, Clasen BF, Nair KS, et al. Fasting increases human skeletal muscle net phenylalanine release and this is associated with decreased mTOR signaling. PLoS One. 2014;9(7):e102031.

97. Ren H, Accili D, Duan C. Hypoxia converts the myogenic action of insulin-like growth factors into mitogenic action by differentially regulating multiple signaling pathways. Proc Natl Acad Sci U S A. 2010;107:5857-62.

98. Arthur PG, Giles JJ, Wakeford CM. Protein synthesis during oxygen conformance and severe hypoxia in the mouse muscle cell line C2C12. Biochim Biophys Acta. 2000;1475(1):83-9.

99. Kadmiel M, Cidlowski JA. Glucocorticoid receptor signaling in health and disease. Trends in pharmacological sciences. 2013;34(9):518-30.

100. Oakley RH, Cidlowski JA. Cellular processing of the glucocorticoid receptor gene and protein: new mechanisms for generating tissue-specific actions of glucocorticoids. J Biol Chem. 2011;286(5):3177-84.

101. Lonard DM, O'Malley B W. Nuclear receptor coregulators: judges, juries, and executioners of cellular regulation. Mol Cell. 2007;27(5):691-700. 
102. Ayroldi E, Cannarile L, Migliorati G, Nocentini G, Delfino DV, Riccardi C. Mechanisms of the anti-inflammatory effects of glucocorticoids: genomic and nongenomic interference with MAPK signaling pathways. FASEB journal : official publication of the Federation of American Societies for Experimental Biology. 2012;26(12):4805-20.

103. Busillo JM, Cidlowski JA. The five Rs of glucocorticoid action during inflammation: ready, reinforce, repress, resolve, and restore. Trends in endocrinology and metabolism: TEM. 2013;24(3):109-19.

104. Hu Z, Wang H, Lee IH, Du J, Mitch WE. Endogenous glucocorticoids and impaired insulin signaling are both required to stimulate muscle wasting under pathophysiological conditions in mice. The Journal of clinical investigation. 2009;119(10):3059-69.

105. Mulligan JD, Gonzalez AA, Kumar R, Davis AJ, Saupe KW. Aging elevates basal adenosine monophosphate-activated protein kinase (AMPK) activity and eliminates hypoxic activation of AMPK in mouse liver. The journals of gerontology Series A, Biological sciences and medical sciences. 2005;60(1):21-7.

106. Clanton TL. Hypoxia-induced reactive oxygen species formation in skeletal muscle. J Appl Physiol (1985). 2007;102(6):2379-88.

107. Li L, Chen Y, Gibson SB. Starvation-induced autophagy is regulated by mitochondrial reactive oxygen species leading to AMPK activation. Cellular signaling. 2013;25(1):50-65.

108. Pouw EM, Schols AM, van der Vusse GJ, Wouters EF. Elevated inosine monophosphate levels in resting muscle of patients with stable chronic obstructive pulmonary disease. American journal of respiratory and critical care medicine. 1998;157(2):453-7.

109. Calvert LD, Singh SJ, Greenhaff PL, Morgan MD, Steiner MC. The plasma ammonia response to cycle exercise in COPD. The European respiratory journal. 2008;31(4):751-8.

110. Sahlin K, Katz A. Hypoxaemia increases the accumulation of inosine monophosphate (IMP) in human skeletal muscle during submaximal exercise. Acta physiologica Scandinavica. 1989;136(2):199-203.

111. Nader N, Ng SS, Lambrou GI, Pervanidou P, Wang Y, Chrousos GP, et al. AMPK regulates metabolic actions of glucocorticoids by phosphorylating the glucocorticoid receptor through p38 MAPK. Molecular endocrinology. 2010;24(9):1748-64.

112. Bouazza B, Debba-Pavard M, Amrani Y, Isaacs L, O'Connell D, Ahamed S, et al. Basal p38 mitogen-activated protein kinase regulates unliganded glucocorticoid receptor function in airway smooth muscle cells. American journal of respiratory cell and molecular biology. 2014;50(2):301-15.

113. Patel J, McLeod LE, Vries RG, Flynn A, Wang X, Proud CG. Cellular stresses profoundly inhibit protein synthesis and modulate the states of phosphorylation of multiple translation factors. Eur J Biochem. 2002;269(12):3076-85.

114. Connolly E, Braunstein S, Formenti S, Schneider RJ. Hypoxia inhibits protein synthesis through a 4E-BP1 and elongation factor 2 kinase pathway controlled by mTOR and uncoupled in breast cancer cells. Mol Cell Biol. 2006;26(10):3955-65. 
115. Jagoe RT, Lecker SH, Gomes M, Goldberg AL. Patterns of gene expression in atrophying skeletal muscles: response to food deprivation. FASEB journal : official publication of the Federation of American Societies for Experimental Biology. 2002;16(13):1697-712.

116. Doucet M, Russell AP, Léger B, Debigaré R, Joanisse DR, Caron M-A, et al. Muscle atrophy and hypertrophy signaling in patients with chronic obstructive pulmonary disease. American journal of respiratory and critical care medicine. 2007;176:261-9.

117. Doucet M, Dube A, Joanisse DR, Debigare R, Michaud A, Pare ME, et al. Atrophy and hypertrophy signaling of the quadriceps and diaphragm in COPD. Thorax. 2010;65(11):963-70.

118. Creutzberg EC, Schols AM, Bothmer-Quaedvlieg FC, Wouters EF. Prevalence of an elevated resting energy expenditure in patients with chronic obstructive pulmonary disease in relation to body composition and lung function. Eur J Clin Nutr. 1998;52(6):396-401.

119. Richalet JP, Letournel M, Souberbielle JC. Effects of high-altitude hypoxia on the hormonal response to hypothalamic factors. American journal of physiology Regulatory, integrative and comparative physiology. 2010;299(6):R1685-92.

120. Izumo S, Nadal-Ginard B, Mahdavi V. All members of the MHC multigene family respond to thyroid hormone in a highly tissue-specific manner. Science. 1986;231(4738):597-600.

121. Mahdavi V, Izumo S, Nadal-Ginard B. Developmental and hormonal regulation of sarcomeric myosin heavy chain gene family. Circulation research. 1987;60(6):804-14.

122. Porter C, Herndon DN, Borsheim E, Chao T, Reidy PT, Borack MS, et al. Uncoupled skeletal muscle mitochondria contribute to hypermetabolism in severely burned adults. Am J Physiol Endocrinol Metab. 2014;307(5):E462-7.

123. Weinberg JM, Venkatachalam MA, Roeser NF, Nissim I. Mitochondrial dysfunction during hypoxia/reoxygenation and its correction by anaerobic metabolism of citric acid cycle intermediates. Proc Natl Acad Sci U S A. 2000;97(6):2826-31.

124. Meyer A, Zoll J, Charles AL, Charloux A, de Blay F, Diemunsch P, et al. Skeletal muscle mitochondrial dysfunction during chronic obstructive pulmonary disease: central actor and therapeutic target. Experimental physiology. 2013;98(6):1063-78.

125. Terzano C, Romani S, Paone G, Conti V, Oriolo F. COPD and thyroid dysfunctions. Lung. 2014;192(1):103-9. 


\section{Summary}


Muscle wasting and weakness is a common but often under-recognized extrapulmonary feature of chronic obstructive pulmonary disease (COPD) that significantly increases disease burden and even mortality risk. Hypoxemia, either chronic or intermittent, is a typical feature of acute or chronic respiratory failure, but surprisingly its potential impact on muscle maintenance in COPD patients is rather unexplored. In chapter $\mathbf{1}$, we critically reviewed available literature addressing the question whether hypoxia may play a causal role in muscle atrophy and impaired muscle oxidative capacity as seen in patients with COPD. Disrupted regulation of muscle mass and oxidative metabolism observed in available experimental animal models resembled observations in muscle biopsies of patients suffering from COPD, suggestive of but not conclusive for a causal role of hypoxia. To address underlying molecular mechanisms, we developed a 21 days normobaric hypoxia ( $8 \%$ oxygen) mouse model in chapter 2 . As hypoxia clearly affected food intake, a pair-fed control group was used for appropriate comparison. We hypothesized that hypoxia-induced muscle atrophy and alterations in the regulation of muscle protein turnover include a hypoxia-specific component, in addition to the observed effects of reduction in food intake in response to hypoxia. Hypoxia induced an initial rapid loss of body and muscle mass, which remained throughout the remainder of the experiment and was partially resulting from hypoxia-induced lowered food intake. Hypoxia induced expression of genes involved in protein degradation signaling such as Ub 26S-proteasome E3 ligases of the ubiquitin proteasome system (UPS) and genes involved in the autophagy-lysosomal pathway (ALP) which could only partially be contributed to hypoxia-induced lowered food intake. Interestingly, mammalian target of rapamycin complex 1 (mTORC1) activity was reduced by the decreased food intake throughout the experiment, but not under hypoxic conditions. These findings suggest a hypoxia-specific impairment of the coordination between protein synthesis and -degradation signaling in skeletal muscle. Loss of muscle mass in COPD patients can largely be attributed to muscle fiber atrophy, particularly of type II fibers. In addition to the loss of muscle mass, peripheral muscles of patients with COPD often are characterized by a shift from slow oxidative type I fibers towards fast glycolytic type II fibers. To investigate whether hypoxia may trigger such events in different muscle types, the adaptations to hypoxia of the oxidative soleus muscle and the glycolytic extensor digitorum longus (EDL) muscle were investigated in chapter 3 . Based on muscle mass and fiber cross section area (FSCA), hypoxia-induced muscle atrophy was more prominent in the EDL than the soleus muscle and only partially resulting from reduced food intake. Despite the lowered FCSA, no shift in fiber type was observed in either muscle type. Hypoxia associated adaptation of gene expression for Hypoxia-induced factor $1 \alpha$ (HIF1 $\alpha$ )-, Glucocorticoid receptor (GR)-, UPS- and ALP signaling were mainly observed in the glycolytic EDL muscle while in the oxidative soleus muscle such 
adaptations were mainly resulting from hypoxia-induced reduction in food intake. The capillary structure was also investigated and hypoxia increased the number of capillary contacts (CC) per $\mu \mathrm{m} 2$ FCSA in both muscles. In the EDL, this was due to type II fiber atrophy, whereas in the soleus the absolute number of $\mathrm{CC}$ increased. Combined these results indicate that glycolytic muscle fibers are more sensitive to hypoxia. As increased glucocorticoid concentrations and GR signaling have also been implicated in muscle atrophy, we hypothesized that hypoxia-induced muscle atrophy is GR dependent in chapter 4. Using a fiber type II specific GR knockout mouse model (mGRKO), we investigated the role of the GR signaling in hypoxia-induced muscle atrophy after 4 days of hypoxia. Corticosteron levels were equally elevated in the hypoxia and pair-fed group in both mouse models, although elevated downstream GR signaling was diminished by GR deficiency. GR deficiency prevented muscle atrophy in the pair-fed group but not in the hypoxic group. GR deficiency blunted elevated ALP signaling in the pair-fed and hypoxia group, whereas elevated UPS signaling was mainly reduced in the pair-fed group only. Reduced food intake associated inhibition of $\mathrm{mTORC} 1$ as seen in chapter 2 is not GR dependent, whereas the lack of mTORC1 inhibition by hypoxia is GR dependent. The deregulation of mTORC1 by hypoxia does not involve AKT1/TSC2 and AKT1/mTOR signaling nor Redd1 and KIf15 expression. In chapter 5, adaptations to hypoxia were combined with 24 hrs fasting as a mouse model for exacerbation in COPD patients. Reduction in muscle mass by fasting was higher under hypoxic conditions although expression of genes involved protein degradation mechanisms such as expression of UPS and ALP related genes were attenuated compared to the pair-fed controls. mTORC1 activity is inhibited by fasting in the pair-fed controls, however under hypoxic conditions, mTORC1 activity was not diminished by fasting. The mechanism by which hypoxia blocks mTORC1 inhibition does not seem to be through Protein kinase B (AKT1)/Tuberous Sclerosis Complex 2 (TSC2) nor AKT1/mTOR signaling as seen in chapter 4. mTORC1 activity under normoxic and hypoxic conditions in response to fasting correlated to AMP-activated protein kinase (AMPK) activity and may involve DNA-damage-inducible transcript 4 (REDD1). It was concluded that the sustained mTORC1 activity through AMPK and elevated protein degradation are responsible for the sensitivity of the muscle to fasting-induced muscle atrophy. To conclude, this thesis shows that systemic hypoxia results in hypoxia-induced muscle atrophy. Glycolytic muscles are more sensitive to hypoxia-induced muscle atrophy. Hypoxia affects the regulation of protein degradation independent of GR signaling, although the exact mechanism remains to be explored. Hypoxia further impaired the inhibition of mTORC1 activity in response to reduced food-intake. This involves GR and AMPK signaling but the exact mechanisms are not fully understood. 
Samenvatting 
Patiënten met Chronische Obstructieve Longziekten (COPD) hebben onherstelbare schade aan de longen door bijvoorbeeld roken of fijn stof. Afhankelijk van de ernst van de ziekte hebben deze patiënten een verminderde longfunctie, waardoor de zuurstofopname beperkt is. Hierdoor kan een tekort aan zuurstof in het bloed worden ontwikkeld, hypoxemie genaamd. Het verlies van spiermassa en spierkracht is ook een kenmerk van patiënten met COPD. Deze effecten op spier niveau hebben een grote invloed op het dagelijks leven van COPD patiënten. Daarnaast is de mate van spiermassaverlies een prognostische factor voor vervroegd overlijden.

In hoofdstuk 1 wordt op basis van de bestaande literatuur besproken of hypoxemie een effect kan hebben op spiermassa en welke onderliggende mechanismes hier mogelijk bij betrokken zijn. Om te onderzoeken hoe hypoxemie de spiermassa regulatie beïnvloed, is er een dier model ontwikkeld om hypoxemie op te wekken. De normale zuurstof concentratie in de lucht op zee niveau is $21 \%$, ook wel normoxische condities genoemd. In het gebruikte diermodel werden muizen onder een conditie van 8\% (hypoxie) zuurstof gebracht voor 21 dagen door de zuurstof te vervangen voor stikstof gas, waardoor de zuurstof concentratie in het bloed verlaagt (hypoxemie).

In hoofdstuk 2 stond de vraag centraal of hypoxemie de aanmaak en afbraak van spiereiwitten kan reguleren, en welke veranderingen in de muis ontstaan door hypoxie met betrekking tot lichaams-, vet- en spier-gewichten en zuurstof waardes in het bloed. Opvallend was dat hypoxie een behoorlijke daling in voedsel inname veroorzaakte, waardoor er een extra normoxische controle groep (PF-groep) muizen in het experiment opgenomen moest worden, waarvan voedsel inname gelijk werd gesteld aan die van de hypoxische muizen.

Hypoxemie veroorzaakte een snelle spiermassa vermindering in de gastrocnemius skeletspier van het achterbeen. Tevens werd de regulatie van eiwit afbraak mechanismen door hypoxemie verhoogd. Beiden effecten werden maar deels verklaard door de verminderde voedselinname, wat duidt op een hypoxie specifiek effect. Opvallend was de bevinding dat de regulatie van eiwit aanmaak (synthese) niet verminderd was onder hypoxische condities, terwijl deze vermindering wel aantoonbaar was bij een gelijke vermindering van voedselinname onder normoxie. Dit betekent dat hypoxemie ervoor zorgt dat de eiwit aanmaaksynthese regulatie gehandhaafd blijt, terwijl ze deze geremd zou moeten worden als aanpassing op de verminderde voedselinname.

Spieren bestaan uit spiervezels van verschillende samenstelling, welke onder meer afhankelijk is van de capaciteit om zuurstof te gebruiken om energie te produceren. Type I en lla vezels zijn spiervezels met veel mitochondriën (energiefabriekjes) en bloedvaten waardoor ze een energie productie hebben waarbij veel zuurstof wordt verbruikt (oxidatief). Oxidatieve type I vezels zijn voornamelijk 
geschikt voor langdurig langzame bewegingen zoals in duursporten het geval is. Type II(b/x) vezels hebben minder mitochondriën en kunnen daardoor beperkt zuurstof gebruiken in hun energie productie. Ze zijn dus vooral aangewezen op de zuurstof onafhankelijke energie productie (glycolyse). Spiermassaverlies in COPD patiënten is vooral te zien in glycolytische type $I(\mathrm{~b} / \mathrm{x})$ vezels, tevens is er een verschuiving in het aantal vezels van oxidatieve type I naar glycolytische type II waargenomen. Om te onderzoeken hoe verschillende type spieren reageren op hypoxemie, hebben we de aanpassingen in de oxidatieve soleus spier (type I en Ila) en glycolytische extensor digitorum longus (EDL) (type Ila en Ilb/x) vergeleken in hoofdstuk 3 . Verlies van spiermassa en spiervezelgrootte door hypoxemie was het sterkst aanwezig in de glycolytische spier, terwijl de oxidatieve spier enkel door de verminderde voeding was aangedaan. De volgorde van moleculen in het genomisch DNA bepalen welke eiwitten gevormd worden, dit gebeurd met tussenkomst van het RNA in de vorm van mRNA. Vooral in de glycolytische spier werd het mRNA verhoogd die direct worden aangestuurd door een hypoxie gevoelig regulator-eiwit Hypoxia induced factor $1 \alpha$ (HIF1a) genaamd. Tevens werd het RNA gelinkt aan de regulatie van eiwitafbraak en was de glucocorticoïd receptor (GR),een stress-sensor, geactiveerd in de spier. Ook is er onderzocht of er aanpassingen zijn van de bloedvaten en contactpunten tussen het bloedvat en de spiervezel. Door de verlaging van de vezelgrootte in de glycolytische spier waren er meer bloedvat contact punten per oppervlakte maat in de vezel. Wellicht kan de vezel door deze aanpassing beter voorzien worden van zuurstof en voedingsstoffen. In de oxidatieve spier was er geen verlaging van de vezelgrootte maar hadden we een verhoging in het aantal contact punten van het bloedvat met de vezel gemeten, hierdoor is ook de oxidatieve vezel beter voorzien van zuurstof en voedingsstoffen. Dus beide spieren zorgen voor aanpassingen waardoor de spier beter aan zuurstof en voedingstoffen kan komen, wel doen ze dit op verschillende manieren.

In hoofdstuk 4 is onderzocht of glucocorticoïden (een hormoon) via de glucocorticoïd receptor (GR) in de spier een rol spelen in de hypoxemie gestimuleerde spierafbraak. De rol van GR is onderzocht door gebruik te maken van een genetisch gemodificeerde muis, hierbij wordt het gen dat codeert voor GR uitgeschakeld waardoor het RNA en het uiteindelijke GR-eiwit niet meer geproduceerd kan worden. Hoewel het verwijderen van GR het spiermassaverlies na verminderde voeding wel voorkwam, was dit niet het geval voor het hypoxie-afhankelijke deel van de spiermassaverlies. De verhoogde eiwit afbraak regulatie was grotendeels GR afhankelijk na verminderd voedsel inname in de PF-groep, maar niet na hypoxemie. Daarentegen was de veranderde eiwit aanmaak regulatie door hypoxemie wel afhankelijk van GR. 
Omdat tijdens perioden van verslechtering van de ziekte de spiermassa van COPD patiënten niet enkel beïnvloed kunnen worden door hypoxemie, maar ook door een acute afname van nutriënten als gevolg van een verminderde eetlust, is in hoofdstuk 5 onderzocht of hypoxemie de effecten van vasten versterkt. Hypoxemie zorgt inderdaad voor een verhoogd spierafbraak tijdens vasten en dit kwam deels door verminderd voedsel inname voorafgaand aan vasten. Ondanks dat het spiermassa verlies na vasten het meest uitgesproken was onder hypoxie, bleek de eiwitafbraak regulatie minder sterk geactiveerd. Daarentegen was de eiwitaanmaak regulatie ook onder deze omstandigheden ook niet verminderd, terwijl dit compleet was uitgeschakeld na vasten onder normoxie. De regulatie van eiwit-afbraak en -aanmaak op dit niveau kan het versterkte verlies van spiermassa onder hypoxie niet verklaren. Echter, verdere bestudering van de aansturing van de eiwitaanmaak regulatie duidden op het eiwit AMPK-activated protein kinase (AMPK) als mogelijk oorzaak voor de veranderde eiwitaanmaak regulatie onder hypoxemie.

Concluderend, blijkt uit de hier beschreven experimenten dat hypoxemie verlies van spiermassa veroorzaakt en dit deels wordt verklaard door de verminderde voedselinname. Glycolytische spieren zijn gevoeliger dan oxidatieve spieren voor verlies van spiermassa door hypoxemie. De verhoogde eiwit afbraak regulatie door hypoxemie is grotendeels GR onafhankelijk. Hypoxemie verandert de eiwitaanmaak regulatie. Hierbij lijken GR en AMPK betrokken, maar dient het exacte mechanisme dient nog te worden ontrafeld. Deze resultaten suggereren dat hypoxemie een belangrijke rol kan spelen in spiermassaverlies in COPD patiënten en bieden aanknopingspunten voor verder onderzoek voor de ontwikkeling van medicijnen die het verlies van spiermassa tegen kunnen gaan. 


\section{Valorisation}




\section{Societal value of the thesis}

Chronic obstructive pulmonary disease (COPD) is a disease characterized by irreversible obstruction of airflow in the lungs and damage of the alveolar walls resulting in reduced gas exchange. Active smoking is the main risk factor for developing COPD, but air pollution, dust and frequent respiratory infections also contribute. According to the World Health Organization, approximately 65 million people worldwide are affected by COPD, and it is estimated that COPD will be the third leading cause of death in 2020. In the Netherlands, according to Statistics Netherlands (CBS), 54.771 persons died of airway-related diseases between 2009 and 2012, of which 25.409 persons due to COPD [1]. On the 1st of January 2011, 361.800 COPD patients were registered in the Netherlands of which 32.500 patients were newly diagnosed that year [2]. COPD becomes manifest at the age of 55 and higher and the prevalence increases with age [2]. In 2010, there were 22.400 hospitalizations of COPD patients with in total 200.000 days in hospital [2].

COPD is not limited to the lungs as patients often encounter extrapulmonary manifestations such as skeletal muscle-wasting and -weakness and most patients suffer from one or more comorbidities. Muscle wasting is a predictor of morbidity and mortality [3-5]. The features of COPD, such as low-grade inflammation, malnutrition, physical inactivity, corticosteroid medication and hypoxia all contribute to the loss of skeletal muscle mass. COPD patients may experience various degrees of oxygen desaturation, which could result in tissue hypoxia. This may occur during exercise (exertional desaturation), during the night (nocturnal desaturation), during disease exacerbations or chronically due to severely impaired lung function, such as in the case of chronic respiratory failure (CRF). Hypoxemia, either chronic or intermittent, is therefore an obvious feature of COPD, but surprisingly its impact on the maintenance of muscle mass is rather unexplored. This thesis investigated whether and how hypoxia contributes to loss of muscle mass. We developed a mouse model and extensively investigated in different muscle types the effects of hypoxia on muscle maintenance.

\section{Innovation}

We extensively described a mouse model of normobaric hypoxia, in which oxygen concentrations in blood, muscle and fat mass were measured. Protein turnover regulation was investigated by focusing on the regulation of protein degradation and synthesis. As hypoxia clearly affected food intake, the inclusion of a pairfed (PF) group allowed us to discriminate hypoxia-specific effects from effects 
resulting from reduced food intake alone. Consequently, hypoxia-specific effects that accompanied additional muscle atrophy included enhanced regulation of protein degradation and surprisingly, sustained mTORC1 activity compared to mere reduced food intake.

Interestingly, despite higher oxidative capacity of the soleus muscle, the glycolytic EDL was more sensitive to hypoxia-induced muscle atrophy. Hypoxia increased expression of hypoxia-inducing factor a (HIF1a)-, glucocorticoid receptor (GR)-, ubiquitin proteasome system (UPS)- and autophagy lysosomal pathway (ALP) signaling mainly in the glycolytic muscle in line with the reduced muscle mass. We speculated that the glycolytic muscle is more sensitive to hypoxia-induced muscle atrophy due to a lower capillary density compared to the oxidative muscle. As GR signaling and plasma corticosterone levels were elevated by hypoxia, GR signaling was further investigated. Whereas the genetic approach to study GR involvement in protein turnover regulation revealed that GR is not essential to induce protein degradation signaling by hypoxia, these studies identified GR as a nodal point in sustained mTORC1 signaling under hypoxic conditions. In addition, impaired AMPK signaling accompanied aberrantly sustained mTORC1 signaling under hypoxic conditions during fasting.

\section{Knowledge utilization}

Similar to other studies [6-8] we showed that hypoxia reduced food intake. Vermeeren et al. [9, 10] showed that COPD patients with exacerbations had a reduced dietary intake of $\sim 40 \%$ when admitted to the hospital. In addition, oxygen levels in exacerbation patients have been shown to drop as low as 35\% (SaO2) indicating that severe hypoxemia may occur during disease flare-ups [11]. COPD patients in general may experience hypoxemic conditions during daily activities which could affect appetite and dietary intake not only during an exacerbation $[12,13]$. The direct effects of oxygen treatment on nutritional intake are rather unexplored although many patients with long-term oxygen treatment (LTOT) are still malnourished based on their low body mass index (BMI) [14]. Combination of oxygen treatment with dietary and nutrient interventions may hold the key to resolve loss of BMI and muscle wasting.

The finding that hypoxia mainly affects glycolytic muscle implicates hypoxia as a putative trigger of muscle atrophy in COPD, as in COPD patients loss of muscle fiber cross section area (FCSA) is mainly seen in the glycolytic type IIX and IIA/ IIX hybrid fibers [15, 16]. Furthermore, the characteristic shift from type I to II fibers observed in muscle of COPD patients, makes them even more vulnerable 
to stimuli that preferentially evoke atrophy of glycolytic muscle fibers, such as hypoxia. Therefore, addition of an endurance component to physical training in addition to resistance exercise within this patient group may be beneficial as it will not only support maintenance of muscle fiber size but also composition, thereby preventing the fiber type switch to more atrophy-prone type II fibers. Currently there is little evidence to support the use of oxygen during training, due to differences in studies and low numbers of participants [17]. Therefore a large study should be commenced combining oxygen treatment with endurance training in order to prevent muscle wasting in COPD patients.

The novel finding that hypoxia impairs protein synthesis regulation is of major interest, especially since retained protein synthesis signaling is observed in nutritionally deprived conditions. This suggests that coupling of protein synthetic responses to nutritional status or other anabolic signals, is impaired under hypoxia. Such impaired coordination between anabolic response and anabolic signals by hypoxia may be responsible for the lack of response to nutritional interventions in exacerbation patients, who did not increase fat free mass (FFM) or muscle mass [9]. Therefore, this effect should further be investigated as actual proteinsynthesis rates were not measured in this thesis. Future experiments should include infusion of stable isotopes- or radioactively labelled amino acids such as phenylalanine or leucine to measure protein synthesis rates.

This thesis showed for the first time that the glucocorticoid receptor (GR) is involved in the impaired protein synthesis regulation by hypoxia, although the exact mechanism remains to be determined. As GR is a transcription factor, a more comprehensive expression analysis in WT and mGRKO muscle following hypoxia could reveal the GR-dependent gene(s) involved in the differential protein synthesis regulation. GR also has actions that do not involve transcriptional effects (non-genomic actions) through protein-protein interactions. Immuno-precipitations (IP) with GR in combination with a target protein array or mass spectrometry could identify the proteins involved in the impaired protein synthesis regulation. Involvement of GR in the impaired protein synthesis regulation by hypoxia could have implications for the treatment of COPD patients using glucocorticoids (GCs). Oral administration of high doses of GCs during an exacerbation will affect muscle mass irrespective of the hypoxic conditions. GC administration combined with the hypoxic conditions and inflammation may further aggravate muscle loss. Refraining patients from GC administration during exacerbations might avoid GC-induced muscle loss. However, in absence of the anti-inflammatory effects of GCs, inflammation-driven muscle atrophy may occure as exacerbations are often accompanied by pulmonary and systemic inflammation [18]. Use of gluco- 
corticoids combined with GR antagonists, such as RU486 or RU40555, does not seem beneficial as they are not specific for skeletal muscle and may therefore affect other tissues. More interesting is the novel field of selective glucocorticoid receptor modulators (SGRMs) [19]. It is assumed that the anti-inflammatory effects of GCs are largely due to GR transrepression mechanisms, while GR transactivation is accountable for the greater part of GC treatment-associated side effects such as muscle wasting [20, 21]. SGRMs exhibit the anti-inflammatory effects but with reduced side effects, such as skin atrophy $[22,23]$. Treatment of COPD patients with SGRMs instead of GCs may therefore protect muscle mass from GC induced muscle atrophy and may result in normal protein synthesis regulation under hypoxic conditions.

Fasting under hypoxic conditions revealed that impaired protein synthesis regulation by hypoxia was accompanied by disrupted AMPK activation. As key regulator of energy homeostasis, activation of AMPK in skeletal muscle leads to increased glucose uptake and elevated fatty acid oxidation [24]. AMPK is regulated by phosphorylation on for instance Threonine 172 by LKB1 in combination with allosteric modulators such as AMP and glycogen which results in a specific activity of AMPK [24]. Future research should address at what level AMPK activation is impaired under hypoxia, for instance by measuring the allosteric modulators AMP and glycogen or by evaluating activating and inhibiting phosphorylation sites of AMPK. In subsequent studies, the causal contribution of impaired AMPK activation to protein synthesis regulation and muscle atrophy in response to hypoxia should be addressed, as it may uncover therapeutic implications of AMPK modulation during hypoxia. The use of for instance AMPK activator AICAR to restore energy homeostasis and protein turnover may have clinical benefits as intervention studies in animals in muscle showed increased AMPK activity, increased glucose uptake [25], elevated glycogen concentration [26], improved mitochondrial morphology [27], enhanced running endurance by $44 \%$ [28] and fiber type shift towards oxidative fibers [29].

In addition to studying the effects on muscle mass, the normobaric hypoxia mouse model also provides a basis for investigating other aspects of muscle dysfunction in COPD, e.g. loss of oxidative phenotype (OXPHEN), as well as the pathobiology of other tissues in COPD or the role of hypoxia in other diseases. As this model results in systemic hypoxia, other tissues including brain, liver, heart, fat, lungs and intestine could be investigated to address the effects of hypoxemia and tissue hypoxia, as these may contribute to the multi organ dysfunction in COPD patients. Currently, already a couple of scientific papers are based on this mouse model: 2 papers published by Gopal et al. and Eurlings et al., which 
used the mouse model to investigate the remodeling of the lungs by hypoxia [30, 31]. Furthermore, a paper by Poels et al. that used the current mouse model looked into ventricular remodeling of the heart [32]. A paper by Slot et al. [33] investigated the effects of hypoxia on the oxidative metabolism in mice of different ages and a paper by van den Borst et al. [34] showed that white adipose tissue of hypoxic mice resulted in a more brown adipose tissue phenotype. The hypoxic model can further be extended to mimic sleep apnea by intermittent hypoxia during sleep (day time).

In conclusion, this mouse model of normobaric hypoxia is a valuable tool for multiple research questions regarding hypoxia-imposed tissue dysfunction. Moreover, besides early recognition of desaturation episodes and the treatment with oxygen, COPD patients may benefit from controlled dietary and nutrient interventions. The novel findings that hypoxia impairs protein synthesis regulation with involvement of GR and AMPK should be further studied and may reveal targets for interventions and a treatment against muscle loss in COPD patients. 


\section{References}

1. Mulder M HFd. Ziekten van de ademhalingswegen totaal. Volksgezondheid Toekomst Verkenning, Nationale Atlas Volksgezondheid. 2014.

2. Boezen HM PD. COPD samengevat. Volksgezondheid Toekomst Verkenning, Nationaal Kompas Volksgezondheid. 2013.

3. Wouters EF. Nutrition and metabolism in COPD. Chest. 2000;117(5 Suppl 1):274S-80S.

4. Mador MJ, Bozkanat E. Skeletal muscle dysfunction in chronic obstructive pulmonary disease. Respir Res. 2001;2(4):216-24.

5. Gray-Donald K, Gibbons L, Shapiro SH, Macklem PT, Martin JG. Nutritional status and mortality in chronic obstructive pulmonary disease. American journal of respiratory and critical care medicine. 1996;153(3):961-6.

6. Kozak W, Wrotek S. Hypoxia-induced sickness behaviour. Pharmacology. 2006:35-50.

7. Chen XQ, Dong J, Niu CY, Fan JM, Du JZ. Effects of hypoxia on glucose, insulin, glucagon, and modulation by corticotropin-releasing factor receptor type 1 in the rat. Endocrinology. 2007;148(7):3271-8.

8. Westerterp-plantenga MS, Westerterp KR, Rubbens M, Verwegen RT, Richelet J-p, Gardette B, et al. Appetite at " high altitude " [ Operation Everest III ( Comex- ' 97 )]: a simulated ascent of Mount Everest effects of caloric restriction J Appl Physiol (1985). 2011:391-9.

9. Vermeeren MA, Wouters EF, Geraerts-Keeris AJ, Schols AM. Nutritional support in patients with chronic obstructive pulmonary disease during hospitalization for an acute exacerbation; a randomized controlled feasibility trial. Clinical nutrition. 2004;23(5):1184-92.

10. Vermeeren MA, Schols AM, Wouters EF. Effects of an acute exacerbation on nutritional and metabolic profile of patients with COPD. The European respiratory journal. 1997;10(10):22649 .

11. Henrion J, Minette P, Colin L, Schapira M, Delannoy A, Heller FR. Hypoxic hepatitis caused by acute exacerbation of chronic respiratory failure: a case-controlled, hemodynamic study of 17 consecutive cases. Hepatology. 1999;29(2):427-33.

12. Soguel Schenkel N, Burdet L, de Muralt B, Fitting JW. Oxygen saturation during daily activities in chronic obstructive pulmonary disease. The European respiratory journal. 1996;9(12):2584-9.

13. Schols A, Mostert R, Cobben N, Soeters P, Wouters E. Transcutaneous oxygen saturation and carbon dioxide tension during meals in patients with chronic obstructive pulmonary disease. Chest. 1991;100(5):1287-92.

14. Chailleux E, Laaban JP, Veale D. Prognostic value of nutritional depletion in patients with COPD treated by long-term oxygen therapy: data from the ANTADIR observatory. Chest. 2003;123(5):1460-6.

15. Gosker HR, Engelen MPKJ, van Mameren H, van Dijk PJ, van der Vusse GJ, Wouters EFM, et al. Muscle fiber type IIX atrophy is involved in the loss of fat-free mass in chronic obstructive pulmonary disease. Am J Clin Nutr. 2002;76:113-9. 
16. Natanek SA, Riddoch-Contreras J, Marsh GS, Hopkinson NS, Moxham J, Man WD, et al. MuRF-1 and Atrogin-1 Protein Expression and Quadriceps Fiber Size and Muscle Mass in Stable Patients with COPD. COPD. 2013;10(5):618-24.

17. Nonoyama ML, Brooks D, Lacasse Y, Guyatt GH, Goldstein RS. Oxygen therapy during exercise training in chronic obstructive pulmonary disease. The Cochrane database of systematic reviews. 2007(2):CD005372.

18. Tkacova R, Kluchova Z, Joppa P, Petrasova D, Molcanyiova A. Systemic inflammation and systemic oxidative stress in patients with acute exacerbations of COPD. Respiratory medicine. 2007;101(8):1670-6.

19. Sundahl N, Bridelance J, Libert C, De Bosscher K, Beck IM. Selective glucocorticoid receptor modulation: New directions with non-steroidal scaffolds. Pharmacology \& therapeutics. 2015;152:28-41.

20. Carballo-Jane E, Pandit S, Santoro JC, Freund C, Luell S, Harris G, et al. Skeletal muscle: a dual system to measure glucocorticoid-dependent transactivation and transrepression of gene regulation. The Journal of steroid biochemistry and molecular biology. 2004;88(2):191201.

21. Schacke H, Docke WD, Asadullah K. Mechanisms involved in the side effects of glucocorticoids. Pharmacology \& therapeutics. 2002;96(1):23-43.

22. Schacke H, Zollner TM, Docke WD, Rehwinkel H, Jaroch S, Skuballa W, et al. Characterization of ZK 245186, a novel, selective glucocorticoid receptor agonist for the topical treatment of inflammatory skin diseases. Br J Pharmacol. 2009;158(4):1088-103.

23. Schacke H, Schottelius A, Docke WD, Strehlke P, Jaroch S, Schmees N, et al. Dissociation of transactivation from transrepression by a selective glucocorticoid receptor agonist leads to separation of therapeutic effects from side effects. Proc Natl Acad Sci U S A. 2004;101(1):227-32.

24. Hardie DG, Ross FA, Hawley SA. AMPK: a nutrient and energy sensor that maintains energy homeostasis. Nat Rev Mol Cell Biol. 2012;13(4):251-62.

25. Iglesias MA, Ye JM, Frangioudakis G, Saha AK, Tomas E, Ruderman NB, et al. AICAR administration causes an apparent enhancement of muscle and liver insulin action in insulinresistant high-fat-fed rats. Diabetes. 2002;51(10):2886-94.

26. Aschenbach WG, Hirshman MF, Fujii N, Sakamoto K, Howlett KF, Goodyear LJ. Effect of AICAR treatment on glycogen metabolism in skeletal muscle. Diabetes. 2002;51(3):567-73.

27. Qi Y, Shang JY, Ma LJ, Sun BB, Hu XG, Liu B, et al. Inhibition of AMPK expression in skeletal muscle by systemic inflammation in COPD rats. Respir Res. 2014;15:156.

28. Narkar VA, Downes M, Yu RT, Embler E, Wang YX, Banayo E, et al. AMPK and PPARdelta agonists are exercise mimetics. Cell. 2008;134(3):405-15.

29. Suwa M, Nakano H, Kumagai S. Effects of chronic AICAR treatment on fiber composition, enzyme activity, UCP3, and PGC-1 in rat muscles. J Appl Physiol (1985). 2003;95(3):960-8. 
30. Eurlings IM, Reynaert NL, van den Beucken T, Gosker HR, de Theije CC, Verhamme FM, et al. Cigarette smoke extract induces a phenotypic shift in epithelial cells; involvement of HIF1alpha in mesenchymal transition. PLoS One. 2014;9(10):e107757.

31. Gopal P, Gosker HR, Theije CC, Eurlings IM, Sell DR, Monnier VM, et al. Effect of chronic hypoxia on RAGE and its soluble forms in lungs and plasma of mice. Biochim Biophys Acta. 2015;1852(5):992-1000.

32. Poels EM, Bitsch N, Slenter JM, Kooi ME, de Theije CC, de Windt LJ, et al. Supplementing exposure to hypoxia with a copper depleted diet does not exacerbate right ventricular remodeling in mice. PLoS One. 2014;9(4):e92983.

33. Slot IG, Schols AM, de Theije CC, Snepvangers FJ, Gosker HR. Alterations in Skeletal Muscle Oxidative Phenotype in Mice Exposed to 3 Weeks of Normobaric Hypoxia. Journal of cellular physiology. 2016;231(2):377-92.

34. van den Borst B, Schols AM, de Theije C, Boots AW, Kohler SE, Goossens GH, et al. Characterization of the inflammatory and metabolic profile of adipose tissue in a mouse model of chronic hypoxia. J Appl Physiol (1985). 2013;114(11):1619-28. 
Abbreviations 
4E-BP1

AA

ACTH

ADP

ALP

AMP

AMPK

ATF4

ATP

ATROGIN-1 / MAFBX

BMI

BNIP3

C/EBP- $\beta$

CA-9

CBS

$\mathrm{CC}$

$\mathrm{CC}$

$\mathrm{CHOP}$

ChREBp

COPD

CREB

CRF

$\mathrm{CRH}$

DDIT4 / REDD1

DMEM

DMEM

EDL

EIF

ER

FBS

FCSA

FKBP51

$\mathrm{FOXO}$

$\mathrm{GC}$

GLUL

GLUT1

GM

GOLD

GR
elF4E-binding protein 1

amino acids

adrenocorticotropic hormone

adenosine diphosphate

autophagy-lysosomal pathway

adenosine monophosphate

AMP-activated protein kinase

activating transcription factor 4

adenosine triphosphate

muscle atrophy F-box

body mass index

BCL2/adenovirus E1B 19 kDa protein-interacting

protein 3

CCAAT/enhancer-binding protein- $\beta$

carbonic anhydrase IX

statistics Netherlands

capillary contacts

corticosterone

CCAAT/enhancer-binding protein homolegues protein carbohydrate response element binding protein chronic obstructive pulmonary disease

cAMP response element-binding

chronic respiratory failure

corticotropin-releasing hormone

DNA-damage-inducible transcript 4 protein

differentiation medium

dulbecco's modified Eagle's medium

extensor digitorum longus

eukaryotic translation initiation factor

endoplasmic reticulum

fetal bovine serum

fiber cross section area

FK506 binding protein 5

forkhead box $\mathrm{O}$

glucocorticoid

glutamate-ammonia ligase

glucose transporter 1

growth medium

Global Initiative for Chronic Obstructive Lung Disease glucocorticoid receptor 


\begin{tabular}{|c|c|}
\hline GRE & glucocorticoid response element \\
\hline $\mathrm{H}$ & hypoxia \\
\hline HIF & hypoxia-inducible factors \\
\hline HNSCC & head and neck squamous cell carcinoma \\
\hline HRE & hypoxia responsive element \\
\hline HSP90 & heat shock protein 90 \\
\hline IGF-1 & insulin growth factor-1 \\
\hline IL-1 $\beta$ & interleukin-1 $\beta$ \\
\hline IL-6 & interleukin 6 \\
\hline IL-8 & interleukin 8 \\
\hline IMP & inosine monophosphate \\
\hline IRS-1 & insulin receptor substrate-1 \\
\hline IKB & $\begin{array}{l}\text { nuclear factor of kappa light polypeptide gene } \\
\text { enhancer in B-cells inhibitor }\end{array}$ \\
\hline KLF15 & krüppel-like factor 15 \\
\hline LTOT & long-term oxygen therapy \\
\hline LVRS & lung volume reduction surgery \\
\hline MAP1LC3 & microtubule-associated protein 1 light chain- 3 \\
\hline MAPK & mitogen-activated protein kinase \\
\hline MCP-1 / CCL2 & monocyte chemotactic protein 1 \\
\hline mGRKO & muscle-specific GR knockout \\
\hline MSTN & myostatin \\
\hline MTOR & mammalian target of rapamycin \\
\hline mTORC1 & mammalian target of rapamycin complex 1 \\
\hline MURF1 / TRIM63 & muscle-specific ring finger 1 \\
\hline $\mathrm{N}$ & normoxia \\
\hline $\mathrm{NF}-\mathrm{kB}$ & nuclear factor kappa-B \\
\hline nGRE & negative GRE \\
\hline NOD & nocturnal oxygen desaturation \\
\hline OXPHEN & oxidative phenotype \\
\hline P70S6K1 & P70 ribosomal protein S6 kinase 1 \\
\hline $\mathrm{PaCO} 2$ & arterial partial pressure of $\mathrm{CO} 2$ \\
\hline $\mathrm{PaO} 2$ & arterial partial pressure of $\mathrm{O} 2$ \\
\hline $\mathrm{PDH}$ & prolyl hydroxylases \\
\hline PF / PN & pair-fed \\
\hline PGC1a & $\begin{array}{l}\text { peroxisome proliferator-activated receptor gamma } \\
\text { coactivator } 1 \text {-alpha }\end{array}$ \\
\hline $\mathrm{PI} 3 \mathrm{~K}$ & phosphatidylinositol-4,5-bisphosphate 3-kinase \\
\hline piO2 & intracellular $\mathrm{O} 2$ partial pressure \\
\hline PKB / AKT1 & protein kinase $\mathrm{B}$ \\
\hline
\end{tabular}


PPAR

PPP1R15A / GADD34

$\mathrm{RBC}$

REE

REEFFM

REM

ROS

rpS6

RT-qPCR

$\mathrm{SaO} 2$

SGRM

SMAD3

SREBP1C

$\mathrm{StO} 2$

$\mathrm{T} / \mathrm{Thr}$

TCA

$\mathrm{TH}$

TNFR1

TNFa

TSC

UB

UCP3

ULK1

UPR

UPS

VEGF

VHL peroxisome proliferator-activated receptor

protein phosphatase 1 , regulatory subunit 15A

red blood cells

resting energy expenditure

REE based on fat-free mass

rapid eye movement

reactive oxygen species

ribosomal protein S6

real-time quantitive PCR

hemoglobin oxygen saturation

selective glucocoricoid receptor modulator

mothers against decapentaplegic homolog 3

sterol regulatory element-binding protein 1c

ratio of oxygenated hemoglobin to total hemoglobin

threonine

tricarboxylic acid

thyroid hormone

tumor necrosis factor receptor 1

tumor necrosis factor $\alpha$

tuberous sclerosis protein

ubiquitin

mitochondrial uncoupling protein 3

UNC51-linke kinase 1

unfolded protein response

ubiquitin-proteasome system

vascular endothelial growth factor

von Hippel-Lindau 
Dankwoord 
Waar moet ik beginnen ... om de mensen te bedanken die mij geholpen hebben om aan dit hoofdstuk toe te komen. Gezien mijn lange tijd hier aan de universiteit van Maastricht zal dat toch vanaf het begin moeten. Dit, aangezien ik door interacties met collega's/vrienden zo gegroeid ben tot wat ik heden ben geworden.

Mijn eerste contact binnen de Universiteit van Maastricht was met Cardioloog Pieter Doevendans in Juni 1999. Ik werd aangenomen om naast Roland Bronsaer te gaan werken aan hart onderzoek binnen het CARIM instituut. Je leerde me hoe de link was tussen de kliniek en de universiteit. Ondanks het grote gat tussen deze twee werelden, maakte je tijd voor mij en je collega's. De NO-gas metingen met een electrode hebben mij destijds (en nog steeds) voor een raadsel gezet. Ik herinner me nog duidelijk dat ik elke dinsdag om precies 13:00 spontaan pieken kreeg en ben er nooit achter gekomen waar die nu vandaan kwamen. Gelukkig kwam Leon de Windt op de afdeling en Leon, ik ben je nog zeer dankbaar dat je mij van het NO-project afgehaald hebt. Samen met de AIO's: Eva van Rooij, Vanessa van Empel en Fawzi Babiker hebben we mooi onderzoek gedaan naar hypertrofie d.m.v. neonatale cardiomyocyten. Jij was het ook, die me zelfstandig methodes liet uitzoeken en mijn eerste introductie gaf in het schrijven. Dit resulteerde in 2 artikelen in Methods in molecular cardiology. Onbegrijpelijk moest ik wel elke dag precies op tijd naar huis van je. Ook hebben we samen de opzet gemaakt tot wat nu de afdeling Moleculaire Cardiologie is geworden. Ik herinner me nog het gesleep van een tafel voor ons eigen labje. We hadden voor die tijd altijd in het lab van moleculaire fysiologie gezeten, met Peter Willemsen aan het roer. Peter, het is alweer enige tijd geleden maar ik ben dankbaar voor de goede zorgen en gezelligheid in het lab en koffiekamer van Fysiologie. Natuurlijk ook mijn dank naar de andere collega's van Fysiologie en Cardiologie voor hun gezelligheid.

Ik solliciteerde in 2002 bij een nieuwe onderzoeksgroep binnen de afdeling Anatomie \& Embryologie. Aangezien Maastricht inmiddels mijn thuis was geworden, ben ik niet met Leon en Pieter mee naar het Hubrecht Instituut in Utrecht vertrokken. Alhoewel ik eerst mijn bedenkingen had over het moleculaire lab met schildpadden, hagedissen en aquaria, werd mij verzekerd dat er een nieuw lab kwam. Gelukkig was de basis die ik met Leon had opgebouwd genoeg om aangenomen te worden door Professor Wout Lamers en Eleonore Köhler. Eleonore, ik kijk met plezier terug op het feit dat je om 5 uur s'ochtends een taart ging bakken en we die dan mochten proeven. Meestal was dit geen straf en eisten we meer, alhoewel ik me niet kan herinneren dat dit ooit gelukt is. Dit is natuurlijk wel de basis geweest voor mijn bakkunsten. Het resulteerde in de bakwedstrijden die de afdeling extra gezellig maakte. Ik heb helaas moeten zien 
hoe je focus van onderzoek snel veranderde in het onderwijs, iets wat in mijn ogen niet ten goede kwam voor het onderzoek. Wout, je bent een bevlogen man die met hart en ziel geniet van de wetenschap in al zijn glorie. Hierdoor heb ik de kans gekregen om inzicht te krijgen in meerdere onderzoeksgebieden zoals astma, endotheel, hersenen, aminozuur -transport en -regulatie in de darm. Zelfs toen we spontaan stuitte op een situs inversus muizen lijn, wilde jij hiermee aan de slag. Eleonore en Wout, ondanks dat ik niet alles accepteerde wat er werd veranderd in mijn artikelen, wat soms nogal voor vertraging zorgde, waardeer ik jullie schrijftalent. Ik heb er dan ook veel van geleerd. Els Terwindt, samen hebben wij met veel plezier de moleculaire tak van de afdeling gemanaged en veel bijgeleerd over moleculair onderzoek. Ik waardeer je steun in de soms wat moeilijke tijden. Paul van Dijk, koning van het histologisch onderzoek, welke oude technieken jij allemaal kan opduikelen is ontelbaar. Met ontzag heb ik gekeken naar het snijden van hele ratten op de cryo-macrotoom en hoe gemakkelijk je even paraffine- of vries- coupes sneed. Ik heb geprobeerd me ver van histologische technieken af te houden, maar heb ontdekt dat dat eigenlijk niet gaat. Ik ben je dankbaar voor de basis die je me hebt aangeleerd. Ook nu nog kan ik altijd aankloppen bij de analisten kamer en met het juiste antwoord en/of product naar mijn afdeling teruggaan. Naast het onderzoek is er ook nog de onderwijs kant van Anatomie \& Embryologie. Arno Lataster, ik ken niemand die zo veel van het menselijk lichaam weet als jij. Hoewel ik af en toe les kreeg in de anatomie van de mens, was ik snel uitgeleerd zodra je met de Latijnse namen kwam aanzetten. Ik heb leuke herinneringen aan onze gezamelijke zoektocht naar fossielen in Euverem. Johan Hekking en Leon Huiberts, dat jullie op één kamer zijn geplaatst is me nog steeds een raadsel. Volgens mij versterken jullie elkaar, wat resulteerde in een hoop onzin die uitgekraamd werd. Ik kon daar wel van genieten en waardeer ook de behulpzaamheid bij al mijn vragen. De afdeling Anatomie \& Embryologie is een warme afdeling met hart voor zijn medewerkers, zoals ik zelf heb mogen ervaren tijdens moeilijke tijden in privé-sfeer. Hiervoor mijn dank aan alle collega's van Anatomie \& Embryologie.

In 2007 kreeg ik de kans om binnen het Nutrim instituut in deeltijd promotieonderzoek te doen naar spierafbraak bij COPD patiënten en daarvoor een muis model te ontwikkelen. Ik heb het gewaardeerd dat ik als voormalig analist opgenomen werd in de AIO groep w.o. Vincent Marion, Selvikumari Sankaranarayan, Roy Cloots, Tine Comhair, Sonia Garcia Caraballo, Ramesh Chennupatti. Menig feestje hebben wij gezamelijk afgelopen. Met name de PhD-parties waren legendarisch en vormen een blijvende herinnering. Tine, ik heb mogen genieten van je onbesuisd zijn en onze gezamenlijke liefde voor Disney films en liedjes, die we eindeloos ten gehore brachten. Dit tot grote frustratie van Sonia die daar 
helemaal niks mee had. Samen met Sonia hadden we ook onze mascottes zoals de budda's en muizen die ook nu nog op mijn werktafel staan. Zeker aan het einde van jullie promotie traject waren we een goed team. De komst van Ramesh zorgde voor veel vertier met telkens weer de vraag of hij getrouwd van zijn vakantie zou terugkomen.

Mijn promotie onderzoek was een samenwerking tussen de afdelingen Pulmonologie en Anatomie \& Embryologie. Professor Annemie Schols werd mijn promotor. Ondanks haar drukke schema met al haar taken van dien, vond ze toch telkens tijd om bij de besprekingen te zitten. Annemie, ik ben blij dat je de druk opgevoerd hebt anders had het afronden van dit proefschrift nog langer geduurd of was het misschien helemaal niet meer tot stand gekomen, ondanks dat dit eigenlijk nooit een optie is geweest. Ook waardeer ik jouw eigenschap om eerst informeel met me te praten over gezin, vakanties, etc., alvorens we met de formele gesprekken begonnen. Naast de samenwerking met Professor Wout Lamers van de afdeling Anatomie \& Embryologie werd Eleonore Köhler toegewezen als copromotor, samen met Ramon Langen van de afdeling Pulmonolgie. Ramon, het was even aftasten wat ik precies van je kon verwachten, maar ben erachter gekomen dat je enorm veel tijd en energie steekt in de ontwikkeling van je AIO's en zo ook in mij. Ik waardeer je geduld waarmee je complexe zaken kan uitleggen en het feit dat je meestal meteen terug mailt om 1:00 s 'nachts. Door de goede samenwerking heb je samen met Annemie ervoor gezorgd dat ik nu bij de afdeling Pulmonologie werk en me verder kan ontplooien in het onderzoek, organisatie en onderwijs. Mijn dank hiervoor.

Bij Pulmonologie is er altijd wel wat te doen, is er even geen werk dan zijn er altijd nog de eindeloze vragen van de AIO's en studenten. Eerlijk gezegd vind ik dat ook het leukst, ondanks dat ik het de laatste tijd wel erg druk in mijn hoofd had om meteen door te hebben waar de vragen over gingen. Anita Kneppers jij maakt me altijd vrolijk, zijn het niet je bizarre vragen dan is het je verschijning in het lab wel. Ook geniet ik erg van de studenten die na een stage bij ons verder doorgroeien als AIO, zoals Pieter Leermakers en Judith Ceelen. Pieter, ondanks de wat moeilijke ochtend na jouw verjaardag ben je toch op tijd op je werk gekomen en dat zegt iets over je doorzetting vermogen (alhoewel we je de rest van de dag niet meer gezien hebben). Verder blijf ik met bewondering meeluisteren (en participeren) naar de meest zinloze discussies die jij start tijdens de lunch. Judith, er zijn personen in je carrière die je blijft onthouden, jij bent er hier zeker één van. Met veel plezier kijk ik terug op je stage tijd toen je bij mij gedropt werd door Ramon, die dacht dat ik je wel aan kon. Je mocht eerst histologie doen voor het GR-artikel, maar ik kreeg al snel in de gaten dat er meer in je schuilde. 
Samen hebben we met veel plezier uitgezocht wat er gebeurd met de SMAD signalering tijdens hypoxie. Je mocht zelfs extra lang blijven, wat Ramon iets meer geld kostte dan gedacht ... het was het meer dan waard. Na je stage in Italië werd je AIO in het Pulmonologie lab en ben je nu aan de laatste loodjes bezig. Besef goed, hoe frustrerend wetenschap ook kan zijn, het eind is in zicht en nu is het gewoon even doorbijten en afmaken. Harry Gosker, helaas heb je maar een kleine rol gespeelt in het afronden van mijn thesis, maar ik weet zeker dat je Wout Lamers zijn wasbak theorie nog lang zal herinneren. Daarbuiten waardeer ik je kennis over eigenlijk alles, wat dan ook menig discussie aanzwengelt, o.a. met Pieter die ook denkt alles te weten. Gelukkig is er zoiets als internet en worden menig discussie snel gesust. lk hoop dat ik in de toekomst nog veel van je mag leren en hoop dat je me ooit wegwijs maakt in de organisatie van het onderwijs. Marco Kelders, samen vormen we de basis van ons lab en zijn we verantwoordelijk voor eigenlijk alles. Je hebt een rustig karakter, dat je siert, en zal niet snel afgeleid zijn door de eindeloze stroom aan vragen van studenten en AIO's. Ik hoop dat we samen nog lang het fundament van dit lab kunnen zijn en de kennis kunnen delen met alle collega's. Verder wil ik iedereen van de afdeling bedanken voor de gezellige tijd.

Ik wil naast alle studenten die over de jaren heen zijn langsgekomen ook speciaal enkele personen binnen de universiteit bedanken, die niet direct naast mij werkzaam waren op de werkvloer, voor het delen van hun kennis en behulpzaamheid zoals: Gert Schaart, Janneau van Kranenburg, Patrick van Gorp, Will Coumans, Bas Boonen, Mohammed Hadfoune, de heren en dames van CPV en de spoelkeuken.

Veel mensen zijn op mijn pad binnen en buiten de universiteit gekomen. Te veel om allemaal bij naam te noemen. Nietemin wil ik iedereen bedanken die een bijdrage heeft geleverd, in welke vorm dan ook, aan dit mooie eindresultaat.

Naast de collega's/vrienden van het werk wil ook mijn (schoon-) familie, vrienden en kennissen bedanken voor de interesse, steun en ontspanning naast het werk.

Ik besef heel goed dat de basis voor dit alles gevormd is door mijn ouders Charles en Nel De Theye. Hun eindeloze zorg en toewijding hebben me de kans gegevens om mijn eigen weg te kiezen. Hierdoor ben ik in Venlo op de Hogeschool terecht gekomen en heb ik daar mijn liefde van mijn leven leren kennen. Nicole, we zijn al 20 jaar een stel en samen hebben we al veel meegemaakt zoals het overlijden van mijn zus, Lonne, en je gevecht tegen die verschrikkelijke ziekte. Gelukkig kunnen we dit nu grotendeels achter ons laten en hebben we 
nu een mooi gezin met 2 prachtige dochters, Meike en Lieke. Weet dat ik enorm veel van je hou en al heb/had ik het erg druk met promoveren, mijn liefde voor jou is er niet minder op geworden. Ik ben blij dat je me geholpen hebt deze promotie af te ronden door mij te steunen, aan te moedigen, naar boven te sturen en in mij te geloven. Dikke kus voor jou en mijn kleine lieve meiden. 
Publications 
C.C. de Theije, A.M.W.J. Schols, W.H. Lamers, S.E. Köhler, R.C.J. Langen. Hypoxia sensitizes skeletal muscle to fasting-induced muscle atrophy and impairs AMPK/mTORC1 signaling in mice. Submitted.

C.C. de Theije, A.M.W.J. Schols, W.H. Lamers, J.J.M. Ceelen, R.H. van Gorp, J.J.R. Hermans, S.E. Köhler, R.C.J. Langen. Hypoxia-induced muscle atrophy and impaired regulation of protein turnover are partially dependent on muscle GR signaling. Submitted.

Slot IG, Schols AM, de Theije CC, Snepvangers FJ, Gosker HR. Alterations in Skeletal Muscle Oxidative Phenotype in Mice Exposed to 3 Weeks of Normobaric Hypoxia. J Cell Physiol. 2016 Feb;231(2):377-92.

Gopal P, Gosker HR, de Theije CC, Eurlings IM, Sell DR, Monnier VM, Reynaert NL. Effect of chronic hypoxia on RAGE and its soluble forms in lungs and plasma of mice. Biochim Biophys Acta. 2015 May;1852(5):992-1000.

Pansters NA, Schols AM, Verhees KJ, de Theije CC, Snepvangers FJ, Kelders MC, Ubags ND, Haegens A, Langen RC. Muscle-specific GSK-3 $\beta$ ablation accelerates regeneration of disuse-atrophied skeletal muscle. Biochim Biophys Acta. 2015 Mar;1852(3):490-506.

de Theije CC, Langen RC, Lamers WH, Gosker HR, Schols AM, Köhler SE. Differential sensitivity of oxidative and glycolytic muscles to hypoxia-induced muscle atrophy. J Appl Physiol (1985). 2015 Jan 15;118(2):200-11.

Podcast: http://jappl.podbean.com/e/differential-sensitivity-of-oxidative-andglycolytic-muscles-to-hypoxia-induced-muscle-atrophy/

Eurlings IM, Reynaert NL, van den Beucken T, Gosker HR, de Theije CC, Verhamme FM, Bracke KR, Wouters EF, Dentener MA. Cigarette smoke extract induces a phenotypic shift in epithelial cells; involvement of HIF1a in mesenchymal transition. PLoS One. 2014 Oct 16;9(10):e107757

Poels EM, Bitsch N, Slenter JM, Kooi ME, de Theije CC, de Windt LJ, van Empel VP, da Costa Martins PA. Supplementing exposure to hypoxia with a copper depleted diet does not exacerbate right ventricular remodeling in mice. PLoS One. 2014 Apr 15;9(4):e92983. 
Verhees KJ, Pansters NA, Baarsma HA, Remels AH, Haegens A, de Theije CC, Schols AM, Gosens R, Langen RC. Pharmacological inhibition of GSK-3 in a guinea pig model of LPS-induced pulmonary inflammation: II. Effects on skeletal muscle atrophy. Respir Res. 2013 Nov 1;14:117.

Op den Kamp CM, Langen RC, Snepvangers FJ, de Theije CC, Schellekens JM, Laugs F, Dingemans AM, Schols AM. Nuclear transcription factor K B activation and protein turnover adaptations in skeletal muscle of patients with progressive stages of lung cancer cachexia. Am J Clin Nutr. 2013 Sep;98(3):738-48.

Cloots RH, Sankaranarayanan S, de Theije CC, Poynter ME, Terwindt E, van Dijk P, Hakvoort TB, Lamers WH, Köhler SE. Ablation of Arg1 in hematopoietic cells improves respiratory function of lung parenchyma, but not that of larger airways or inflammation in asthmatic mice. Am J Physiol Lung Cell Mol Physiol. 2013 Sep;305(5):L364-76.

Marion V, Sankaranarayanan S, de Theije C, van Dijk P, Hakvoort TB, Lamers WH, Köhler ES. Hepatic adaptation compensates inactivation of intestinal arginine biosynthesis in suckling mice. PLoS One. 2013 Jun 13;8(6).

de Theije CC, Langen RC, Lamers WH, Schols AM, Köhler SE. Distinct responses of protein turnover regulatory pathways in hypoxia- and semistarvation-induced muscle atrophy. Am J Physiol Lung Cell Mol Physiol. $2013 \mathrm{Jul}$ 1;305(1):L82-91.

van den Borst B, Schols AM, de Theije C, Boots AW, Köhler SE, Goossens GH, Gosker HR. Characterization of the inflammatory and metabolic profile of adipose tissue in a mouse model of chronic hypoxia. J Appl Physiol (1985). 2013 Jun;114(11):1619-28.

de Theije C, Costes F, Langen RC, Pison C, Gosker HR. Hypoxia and muscle maintenance regulation: implications for chronic respiratory disease. Curr Opin Clin Nutr Metab Care. 2011 Nov;14(6):548-53.

Marion V, Sankaranarayanan S, de Theije C, van Dijk P, Lindsey P, Lamers MC, Harding HP, Ron D, Lamers WH, Köhler SE. Arginine deficiency causes runting in the suckling period by selectively activating the stress kinase GCN2. $J$ Biol Chem. 2011 Mar 18;286(11):8866-74. 
He Y, Hakvoort TB, Köhler SE, Vermeulen JL, de Waart DR, de Theije C, ten Have GA, van Eijk HM, Kunne C, Labruyere WT, Houten SM, Sokolovic M, Ruijter JM, Deutz NE, Lamers WH. Glutamine synthetase in muscle is required for glutamine production during fasting and extrahepatic ammonia detoxification. J Biol Chem. 2010 Mar 26;285(13):9516-24. doi:

Schnater JM, Bruder E, Bertschin S, Woodtli T, de Theije C, Pietsch T, Aronson DC, von Schweinitz D, Lamers WH, Köhler ES. Subcutaneous and intrahepatic growth of human hepatoblastoma in immunodeficient mice. J Hepatol. 2006 Sep;45(3):377-86.

de Theije CC, de Windt LJ, Doevendans PA. Methods in molecular cardiology: proteome analysis. Neth Heart J. 2003 Feb;11(2):77-83.

van Rooij E, Doevendans PA, de Theije CC, Babiker FA, Molkentin JD, de Windt LJ. Requirement of nuclear factor of activated T-cells in calcineurin-mediated cardiomyocyte hypertrophy. J Biol Chem. 2002 Dec 13;277(50):48617-26.

de Theije CC, de Windt LJ, Doevendans PA. Methods in molecular cardiology: DNA sequencing. Neth Heart J. 2002 Mar;10(3):136-144. 
Curriculum Vitae 


\section{Curriculum Vitae}

Chiel De Theye was born on April 27th, 1977 in Helmond. He attended the Dr. Knippenberg College in Helmond, where he graduated in 1996. The same year he started the study Technical Microbiology at the Higher Laboratory Education (HLO) in Venlo. In his last year he did an internship called "Investigating the relationship between vancomycin resistant Enterococci in hospitals and other sources " at the University of Plymouth in Great Britain. After graduation in Technical Microbiology in 1999, he went to the Cardiology department at Maastricht University in the lab of the cardiologist Pieter Doevendans where he started as a molecular technician on several studies related to heart failure. He performed many molecular techniques in rat cardiomyocytes cells under supervision of Dr. Leon de Windt and he obtained his artikel 9 certificate for performing animal experiments. In 2002 he started as a molecular technician at the department of Anatomy \& Embryology at Maastricht University in the lab of Prof. Dr. Wout Lamers under supervision of Dr. Leo Köhler. His main focus was amino acid regulation, in particular arginine, in animal studies. He designed and produced a cationic amino acid transporter-1 conditional knock-out mouse which was used in several studies. After 5 years of research assistance he started part-time his PhD in 2007 within a collaboration of the departments Anatomy \& Embryology and Respiratory Medicine where he investigated the regulation of protein turnover in a hypoxia-induced muscle atrophy model. Currently he's working at the department of Respiratory Medicine as senior technician with additional tasks such as teaching and managment of the general ML-II facility. 